\title{
Composition theorems for paired Lagrangian distributions
}

\author{
Dissertation \\ zur Erlangung des mathematisch-naturwissenschaftlichen Doktorgrades \\ "Doctor rerum naturalium" \\ der Georg-August-Universität Göttingen
}

vorgelegt von

Nhu Thang Nguyen

aus Bac Ninh

Göttingen 2011 
Referent: Prof. Dr. Ingo Witt

Korreferentin: Prof. Dr. Dorothea Bahns

Tag der mündlichen Prüfung: 


\section{Abstract}

The theory of Lagrangian distributions and clean compositions of Fourier integral operators, the core of micro-local analysis, was established in the 1970s by the classical works of Hörmander, Duistermaat and Guillemin. Recently, researchers have shown increasing interest in the theory of paired Lagrangian distribution as the natural generalization of Lagrangian distributions. However, an analogous composition theory for paired Lagrangian distributions has not been fully investigated.

The aim of this research is to determine whether the composition of paired Lagrangian distributions belongs to well-known classes such as marked Lagrangian, isotropic Lagrangian, paired Lagrangian or generalized Lagrangian distributions associated with a clean system of closed conic Lagrangian manifolds. By given a new characterization of paired Lagrangian distributions via multi-phase functions, we obtain the first result on strong composition of FIOs and PLDs. As our second result, we show that composed operators stay in the appropriate class under a certain assumption. As a corollary, we achieve the closedness of the classes of paired Lagrangian distributions under composition.

Moreover, we realise an important fact that the failure of this condition can produce new singularities. These singularities are detected in our models for compositions. Finally, we also discuss the weak composition laws for others distributions. 


\section{Zusammenfassung}

Die Theorie der Lagrange-Distributionen und der sauberen Komposition von Fourier-Integraloperatoren, die Kernelemente der mikrolokalen Analysis, wurde in den 1970er Jahren durch die klassischen Arbeiten von Hörmander, Duistermaat und Guillemin begründet. Gepaarte Distributionen können als Verallgemeinerung von Lagrange-Distributionen betrachtet werden. Obschon seit geraumer Zeit im Blickfeld der Wissenschaft, wurde bislang noch keine analoge Kompositionstheorie entwickelt.

Das Ziel dieser Arbeit ist es zu bestimmen, ob die Kompositionen gepaarter Lagrange-Distributionen miteinander oder mit FourierIntegraloperatoren wieder $\mathrm{zu}$ den wohlbekannten Klassen von Distributionen gehören, d.h. ob sie markiert Lagrangesch, isotropisch Lagrangesch, gepaart Lagrangesch oder verallgemeinert Lagrangesch bezüglich sauberen Systemen geschlossen konischer Lagrange Mannigfaltigkeiten sind. Eine neue Charakterisierung von gepaarten LagrangeDistributionen mittels Multiphasenfunktionen liefert ein erstes Resultat über die starke Komposition von Fourier-Integraloperatoren mit gepaarten Lagrange-Distributionen. Weiter kann gezeigt werden, dass die Verknüpfung zweier gepaarter Lagrange-Distributionen unter zusätzlichen Voraussetzungen zu einer der obigen Klassen gehört. Als Korollar erhält man die Geschlossenheit der gepaarter Lagrange-Distributionen unter Verknüpfung.

Darüber hinaus machen wir die wichtige Beobachtung, dass der Wegfall der obigen Voraussetzung Singularitäten erzeugt. Zuletzt diskutieren wir noch die schwache Verknüpfung anderer Distributionen. 


\section{Acknowledgments}

I would like to express my deepest gratitude to Prof. Dr. Ingo Witt, who led me into this interesting problem, and worked with me closely throughout my time in Göttingen. I am indebted to Prof. Witt for his patience, encouragement, insights, and enthusiasm. I would also like to thank Prof. Dr. Dorothea Bahns for her support, understanding and kindness during my study. This research could not have been done without their guidances.

I would also like to thank other members of the Graduate seminar "Analysis of Partial Differential Equations", who provided many helpful comments on the draft versions of this thesis.

I especially thank my family for their steady and limitless love and support, my wife for her infinite encouragement during the difficult times.

Finally, this work would not have been possible without the financial and scientific support of the Research Training Group 1493 "Mathematical Structures in Modern Quantum Physics". 



\section{Contents}

Abstract i

Acknowledgments iii

0 Introduction 1

0.1 Motivation . . . . . . . . . . . . . . . . . . 1

0.2 Formulation of composition theorems $\ldots \ldots \ldots \ldots$

0.3 Main results . . . . . . . . . . . . . . . . . . . . . 7

$1 \quad$ Paired Lagrangian distributions 9

1.1 Well-known results about Fourier integral operators . . . . . . . . . . 9

L.l. Basic definitions . . . . . . . . . . . . . . 9

1.1 .2 Composition of canonical relations f . . . . . . . . 10

L.1.3 Phase functions . . . . . . . . . . . . . . . . . 13

1.1.4 Spaces of amplitude functions . . . . . . . . . . . . . . . 16

1.1 .5 Local representation of Lagrangian distributions $\ldots \ldots$

1.1 .6 Global definition of Lagrangian distributions $\ldots \ldots$. . . . . . . . 18

1.2 Multiphase functions $\ldots \ldots \ldots \ldots$

1.2.1 Definition and geometric interpretation . . . . . . . . . . . 19

1.2 .2 The equivalence of multiphase functions . . . . . . . . . . 25

1.2.3 Reduction of multiphase functions . . . . . . . . . . . . 31

1.3 Amplitude functions of double orders $\ldots \ldots \ldots$

1.3 .1 Definition and basic properties of double symbols . . . . . . . . . 34

1.3 .2 Topology of double symbol spaces . . . . . . . . . . . . . . . 35

1.3.3 Admissible transformations . . . . . . . . . . . . . . 37

1.3.4 Asymptotic expansions of double symbols . . . . . . . . . . . . . 38

1.4 Local representation of paired Lagrangian distributions . . . . . . . . . . 40 
1.4.1 Local definition and micro-local properties . . . . . . . . . . 40

1.4 .2 Oscillatory integral representation of PLDs . . . . . . . . . . . 49

2 Compositions of paired Lagrangian distributions 53

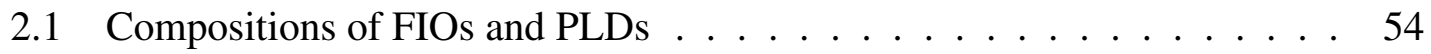

2.1. Geometric condition . . . . . . . . . . . . . 54

2.1 .2 Weak compositions . . . . . . . . . . . . 56

2.1 .3 Strong compositions . . . . . . . . . . . . . . 58

2.2 Compositions of PLDs near their intersections . . . . . . . . . . . 61

2.2.1 Geometric condition . . . . . . . . . . . . . . 61

2.2 .2 Geometric method for weak compositions . . . . . . . . . . 68

2.3 Models for compositions . . . . . . . . . . . . . . . . 81

2.3 .1 Models for strong compositions . . . . . . . . . . . 81

2.3 .2 Models for compositions without the CIS condition . . . . . . . 86

\begin{tabular}{ll}
\hline A Appendix & 93
\end{tabular}

A.1 Distribution theory . . . . . . . . . . . . . . . 93

A.2 Gauss transforms . . . . . . . . . . . . . . . . . . . . . 96

A.3 Marked Lagrangian distributions $\ldots \ldots \ldots$. . . . . . . . . . . . . 97

A.4 The four-Lagrangian distributions f . . . . . . . . . . . . . . 101

$\begin{array}{lr}\text { Bibliography } & 109\end{array}$ 


\section{Introduction}

\subsection{Motivation}

We shall be concerned with the Cauchy problem

$$
\left\{\begin{aligned}
P u & =f & & \text { in } X, \\
\gamma_{0} B_{j} u & =g_{j} & & \text { on } X_{0} ; 1 \leq j \leq v,
\end{aligned}\right.
$$

where $X$ is a $C^{\infty}$ manifold with boundary $X_{0}, \operatorname{dim} X=1+n, P \in L^{m}(X), B_{1} \in L^{m_{1}}(X), \ldots$, $B_{v} \in L^{m_{v}}(X)$ are classical pseudodifferential operators, $\gamma_{0}: C^{\infty}(X) \rightarrow C^{\infty}\left(X_{0}\right)$ is the restriction map, and $f \in \mathcal{E}^{\prime}(X), g_{1}, \ldots, g_{v} \in \mathcal{E}^{\prime}\left(X_{0}\right)$ are the given data.

We shall assume further that $P, B_{1}, \ldots, B_{v}$ have real principal symbols $p(x, \xi)$ and $b_{1}(x, \xi), \ldots, b_{v}(x, \xi)$, respectively, and that $P$ is strictly hyperbolic of multiplicity $v$ with respect to $X_{0}$. In local coordinates, one has $x=(t, y) \in \mathbb{R}_{+}^{1+n}$, where $t>0, y \in \mathbb{R}^{n}$, as well as $P=P\left(t, y, D_{t}, D_{y}\right)$ with principal symbol $p(t, y, \tau, \eta)$, and the strict hyperbolicity of $P$ means that

$$
p_{m}(t, y, \tau, \eta)=a(t, y, \tau, \eta) \prod_{k=1}^{v}\left(\tau-\lambda_{k}(t, y, \eta)\right),
$$

where $\lambda_{j}(t, y, \eta)$ is homogeneous in $\eta$ of degree $1, a(t, y, \tau, \eta) \neq 0$ for all $(t, y, \tau, \eta) \in$ $\left(\overline{\mathbb{R}}_{+}^{1+n} \times \mathbb{R}^{1+n} \backslash 0\right)$ and $\lambda_{1}(t, y, \eta)<\lambda_{2}(t, y, \eta)<\ldots<\lambda_{v}(t, y, \eta)$.

\section{The homogeneous problem}

The well-posedness of the initial-value problem (0.1) in the scale of $L^{2}$-based Sobolev spaces can be investigated with the help of energy inequalities. Another approach consists of constructing an asymptotic solution to the homogeneous problem (i.e., when $f \equiv 0$ ) as a sum of Fourier integral operators. In particular, when $X=\mathbb{R}_{+}^{1+n}, P$ is a differential operator of order $m, B_{j}=\partial_{t}^{j-1}$ for $1 \leq j \leq v$, and $v=m$, this follows from work by Lax [Lax57], Ludwig [Lud60), and Chazarain [Cha74]. More specifically, when combined 
with finite propagation speed, one obtains a unique solution $u \in H_{\mathrm{loc}}^{s+m-1}\left(\mathbb{R}_{+}^{1+n}\right)$ to (0.D) provided that $f \in H_{\mathrm{loc}}^{s}\left(\mathbb{R}_{+}^{1+n}\right), g_{j} \in H_{\mathrm{loc}}^{s+m-j}\left(\mathbb{R}^{n}\right), 1 \leq j \leq m$. Moreover, this solution u can be written in the form

$$
u=E f+\sum_{j=1}^{m} E_{j} g_{j},
$$

where $E_{j}$ are certain Fourier integral operators and $E$ is obtained from $E_{m}$ through an application of Duhamel's principle, see [Cha74]. Note that $E$ is not a Fourier integral operator.

We summarize here the classical results of Lax and Ludwig in the light of the theory of Fourier integral operators. To this end, we assume that the following conditions are met (recall that the bicharacteristic curves of $P$ are the trajectories in $T^{*}(X) \backslash 0$ of the Hamiltonian vector field $H_{p}$ along which the principal symbol $p$ vanishes):

1. Every bicharacteristic curve of $P$ intersects $\left(T^{*}(X) \backslash 0\right)_{X_{0}}$ at most once. Moreover, for every compact set $K \subset T^{*}(X) \backslash 0$ there is a compact set $K_{0} \subset\left(T^{*}(X) \backslash 0\right)_{X_{0}}$ such that every bicharacteristic curve starting in $K$ and hitting $\left(T^{*}(X) \backslash 0\right)_{X_{0}}$ does so in $K_{0}$.

2. No bicharacteristic curve of $P$ starting over $X_{0}$ stays in a compact set and, for all compact sets $K_{0} \subset\left(T^{*}(X) \backslash 0\right)_{X_{0}}$ and $K \subset T^{*}(X) \backslash 0$, there is a compact set $K^{\prime} \subset$ $T^{*}(X) \backslash 0$ with the property that if $\gamma$ is an interval on a bicharacteristic curve of $P$ with end points in $K_{0}$ and $K$, respectively, then $\gamma \subset K^{\prime}$.

Theorem 0.1. Let the above assumptions be fulfilled. Assume further that, in local coordinates $(t, y)$, for all $(y, \eta) \in T^{*} X_{0} \backslash 0$, the matrix $\left(b_{j}\left(0, y, \lambda_{j}(0, y, \eta), \eta\right)\right)_{j, k=1}^{v}$ is non-singular. Then one has a solution formula as in (0.3) (up to a regularizing remainder, the upper summation index being $v$ again), where

$$
E_{j} \in I^{-m_{j}-1 / 4}(C), 1 \leq j \leq v,
$$

and $C \subset\left(T^{*} X \backslash 0\right) \times\left(T^{*} X_{0} \backslash 0\right)$ is the canonical relation that is obtained by jumping down onto one bicharacteristic curve of $P$ and then following the Hamiltonian flow along this bicharacteristic curve.

\section{The free space problem}

Historically, the next problem was to determine the analytic nature of the operator $E$. This was done by taking the $g_{j}$ to be zero, i.e., the free space problem was considered (e.g., by working on the double $2 X$ ). 
It is now possible to assume that $P$ is of real-principal type. Microlocally, this assumption means that $H_{p}$ and the radial vector field $\xi \partial_{\xi}$ (or, equivalently, $d p$ and the canonical 1 -form $\xi d x$ ) are nowhere collinear. Under some further technical conditions of global nature, replacing (1), (2) above, the results are as follows:

Let $\Delta=\Delta_{T^{*}(X) \backslash 0}$ be the diagonal in $\left(T^{*}(X) \backslash 0\right)^{2}$, i.e., the canonical relation associated with the conormal bundle $N^{*}\left(\Delta_{X}\right) \backslash 0$. Let $\Lambda$ be the flow out from $\Lambda \cap(p \pi)^{-1}(0)$ under the Hamiltonian flow of $H_{p}$ for positive time, where $\pi:\left(T^{*}(X) \backslash 0\right)^{2} \rightarrow T^{*}(X) \backslash 0$ is the projection onto the first factor. Note that $\Delta$ is a homogeneous canonical relation, with boundary $\partial \Lambda$, which cleanly intersects $\Delta$ in $\partial \Lambda=\Delta \cap(p \pi)^{-1}(0)$. In [DH72], Duistermaat and Hörmander constructed global parametrices for $P$ by conjugating $P$ with an elliptic Fourier integral operator into the normal form $i^{-1} \partial / \partial x^{1}$, where the latter is easily solved. This construction was turned into a symbolic (or geometric) one by Melrose and Uhlmann [MU79], who defined a general class of distributions associated to two Lagrangian manifolds that intersect cleanly in a submanifold of codimension $k=1$. This was further developed by Guillemin and Uhlmann in [GU81] and many others.

Eventually, in Joshi's thesis [Jos94], [Jos98], these constructions were given in the form of a calculus $I(\Delta, \Lambda)=\cup_{m, p} I^{m, p}(\Delta, \Lambda)$, where now the operators involved admit classical symbols along both Lagrangians $\Delta$ and $\Lambda$. Note that $I^{m, p}(\Delta, \Lambda) \subset I^{m}(\Delta \backslash \Lambda) \cup$ $I^{p}(\Lambda \backslash \Delta)$.

Theorem 0.2. For $P \in I^{m, p}(\Delta, \Lambda)$, there exists a two-side parametrix in $I^{-m,-p-1}(\Delta, \Lambda)$ if and only if $P$ is elliptic in $I^{m, p}(\Delta, \Lambda)$.

As a corollary, one obtains that if $P \in L^{m}(X)$ is a classical pseudodifferential operator, then strict hyperbolicity of $P$ implies ellipticity of $P$ as an element of $I^{m, m-3 / 2}(\Delta, \Lambda)$. Therefore, we then find a parametrix $Q \in I^{-m, 1 / 2-m}(\Delta, \Lambda)$ to $P$ (that, moreover, can be constructed in a symbolic way starting by inverting the principal symbols of $P$ as an element of $I^{m, m-3 / 2}(\Delta, \Lambda)$.

\section{Both constructions combined}

To obtain a symbolic parametrix construction for the inhomogeneous Cauchy problem (1), one needs to combine the results and methods of both approaches above. Formally, the solution $\mathrm{u}$ of the Cauchy problem with source $f \in \mathcal{E}^{\prime}(X)$ and initial data $g_{j} \in \mathcal{E}^{\prime}\left(X_{0}\right)$ 
can be written up to smooth terms as

$$
u=\left(Q-\sum_{j=1}^{v} E_{j} \gamma_{0} B_{j} Q\right) f+\sum_{j=1}^{v} E_{i} g_{j} .
$$

i.e., one has $E=Q-\sum_{j=1}^{v} E_{j} \gamma_{0} B_{j} Q$ in (0.3). This formula is analogous to the solution formula for elliptic boundary value problems (where the free space solution is corrected by a contribution coming from the boundary, given by a so-called singular Green operator).

Note that we already know that

$$
Q \in I^{-m, 1 / 2-m}(\Delta, \Lambda) ; E_{j} \in I^{-m_{j}-1 / 4}\left(X, X_{0} ; C\right) ; \gamma_{0} \in I^{1 / 4}\left(X_{0}, X ; R\right), B_{j} \in L^{m_{j}}(X)
$$

where $R$ is the canonical relation associated to $N_{X \times X_{0}}^{*}\left(\Delta_{X_{0}}\right) \backslash 0$ and $I^{m_{j}}(\Delta)=L^{m_{j}}(X)$. Part of the project is to establish the analytic nature of all the operators and compositions that occur in (0.4). Until now, there is no general theory that encompasses all the compositions required.

\subsection{Formulation of composition theorems}

Let $X, Y, Z$ be smooth manifolds and $A: C_{c}^{\infty}(Y) \rightarrow \mathcal{D}^{\prime}(X), B: C_{c}^{\infty}(Z) \rightarrow \mathcal{D}^{\prime}(Y)$ be linear continuous operators. By abstract composition [Hör85, Theorem 8.2.14], [Dui96, Theorem 1.3.7], or Appendix, Theorem [A.10, whenever $\mathrm{WF}_{Y}^{\prime}(A) \cap \mathrm{WF}_{Y}(B)=\emptyset$ and the projection

$$
\operatorname{supp} k_{A} \times \operatorname{supp} k_{B} \bigcap X \times \Delta_{Y} \times Z \rightarrow X \times Z
$$

is proper, $A \circ B$ is well-defined as an operator from $C_{c}^{\infty}(Z)$ to $\mathcal{D}^{\prime}(X)$. Moreover, wave front relation of the composite is bounded in terms of those of $A$ and $B$

$$
\mathrm{WF}^{\prime}(A \circ B) \subseteq\left(\mathrm{WF}^{\prime}(A) \circ \mathrm{WF}^{\prime}(B)\right) \bigcup\left(\mathrm{WF}_{X}(A) \times 0_{Z}\right) \bigcup\left(0_{X} \times \mathrm{WF}_{Y}^{\prime}(B)\right) .
$$

Question 1. For given admissible operators $A, B$, which further analytic structures the composition $A \circ B$ possesses?

We recall that an operator is admissible if its Schwartz kernel belongs to distributional classes listed in Table W. Such distributions share the following common property: For a given admissible object $G$, the class of distributions associated with $G$, denote by $I(X ; G)$, consists of all distribution $u$ such that $\mathrm{WF}(u)$ is contained in $G$ and $u$ has a special behaviour microlocally at $G$. For instance, if $G$ is a closed conic Lagrangian manifold, then 
$I(X ; G)$ is the class of Lagrangian distributions associated with $G$. The "special behaviour" above means that $u$ can be written as an oscillatory integral via phase function and amplitude function. Similarly, if $G$ is a cleanly intersecting pair $\left(\Lambda_{0}, \Lambda_{1}\right)$ of Lagrangian manifolds then the corresponding class $I\left(X ; \Lambda_{0}, \Lambda_{1}\right)$ is the class of paired Lagrangian distributions and the "special behaviour" stands for oscillatory integral representation in terms of multiphase function and suitable amplitude function. For more detailed discussions about paired Lagrangian distributions, see Chapter $\mathbb{W}$. Other distributions are considered in Chapter [.

\begin{tabular}{|c|c|c|}
\hline Admissible object in $T^{*} X$ & Distributional classes in $X$ & References \\
\hline $\begin{array}{l}\text { Closed conic Lagrangian } \\
\text { manifolds }\end{array}$ & Lagrangian distributions & $\begin{array}{l}\text { [Hör71], [DH72], } \\
\text { [Hör85] }\end{array}$ \\
\hline $\begin{array}{l}\text { Closed conic isotropic man- } \\
\text { ifolds }\end{array}$ & Isotropic distributions & {$[\mathrm{BG81}]$} \\
\hline $\begin{array}{l}\text { Lagrangian manifold with } \\
\text { isotropic marking }\end{array}$ & Marked Lagrangian distributions & [Mel87] \\
\hline $\begin{array}{l}\text { Cleanly intersecting pair of } \\
\text { Lagrangian manifolds }\end{array}$ & Paired Lagrangian distributions & $\begin{array}{l}\text { [MU79], [GU81], } \\
\text { [Men82], [.os98] }\end{array}$ \\
\hline $\begin{array}{l}\text { Clean intersecting system of } \\
\text { Lagrangian manifolds }\end{array}$ & $\begin{array}{l}\text { Generalized Lagrangian distribu- } \\
\text { tions }\end{array}$ & Appendix \\
\hline
\end{tabular}

Table 1: Geometric objects and their related distributions

A relation $G$ from $T^{*} Y \backslash 0$ to $T^{*} X \backslash 0$ is admissible if its twisted $G^{\prime}$ is an admissible object in $T^{*}(X \times Y)$. Let $G_{1}, G_{2}$ be admissible relations from $T^{*} Y \backslash 0$ to $T^{*} X \backslash 0$ and from $T^{*} Z \backslash 0$ to $T^{*} Y \backslash 0$, respectively. Denote by $I\left(X, Y ; G_{1}\right), I\left(Y, Z ; G_{2}\right)$ the classes of properly supported operators associated with $G_{1}, G_{2}$. So composition theorem for these classes can be rewritten in the following form:

Question 2. For given admissible classes of operators $A \in I\left(X, Y ; G_{1}\right)$ and $B \in I\left(Y, Z ; G_{2}\right)$, under which conditions one has

$$
A \circ B \in I(X, Z ; G)
$$

for some admissible relation $G$ ? 
By calculation of wave front relation, one gets $\operatorname{WF}^{\prime}(A \circ B) \subseteq G_{1} \circ G_{2}$. Hence, a complete answer to this question requires the following three parts:

- The geometric condition: Find a condition which ensures the admissibility of $G_{1} \circ$ $G_{2}$.

- The weak law of composition:

$$
I\left(X, Y ; G_{1}\right) \circ I\left(Y, Z ; G_{2}\right) \subseteq I\left(X, Z ; G_{1} \circ G_{2}\right) .
$$

- The strong law of composition:

$$
I^{\mu_{1}}\left(X, Y ; G_{1}\right) \circ I^{\mu_{2}}\left(Y, Z ; G_{2}\right) \subseteq I^{\mu}\left(X, Z ; G_{1} \circ G_{2}\right),
$$

with multi-orders $\mu_{1}, \mu_{2}, \mu$. Moreover, symbolic calculus of such classes holds.

\section{Weak and strong composition theorems}

The weak composition $I\left(X, Y ; G_{1}\right) \circ I\left(Y, Z ; G_{2}\right)$ includes the first two parts: geometric condition and weak law (0.6). In addition, if the inclusion (0.7) holds then one obtains a strong composition of these classes.

Until now, only compositions of Lagrangian distributions and isotropic distributions are well understood. As an illustration for this procedure, let consider composition theorem for Fourier integral operators. Assume that $A \in I\left(X, Y ; C_{1}\right)$ and $B \in I\left(Y, Z ; C_{2}\right)$ are Fourier integral operators associated with canonical relations $C_{1}, C_{2}$. The well-known cleanly condition " $T^{*} X \times \Delta_{T^{*} Y} \times T^{*} Z$ and $C_{1} \times C_{2}$ intersect cleanly with excess $e$ " is the geometric condition for this composition. The geometric part is stated as in [Hör85, Theorem 21.2.14] or Theorem [L.3 in the next chapter. Both weak and strong compositions for Fourier integral operators hold. Namely, if the cleanly intersecting condition of canonical relations is satisfied, then locally $C_{1} \circ C_{2}$ is a canonical relation and $A \circ B \in I\left(X, Z ; C_{1} \circ C_{2}\right)$. Moreover, if $A$ and $B$ are classical of orders $m_{1}, m_{2}$, then $A \circ B$ is a classical FIO of order $m=m_{1}+m_{2}+e / 2$, whose principal symbol can be represented via those of $A, B$. For further informations, see [Hör85, Theorem 25.2.3] or the work of Duistermaat and Guillemin [DG75]. Similar composition theorem holds for classes of isotropic distributions on metaplectic manifolds, see [BG81]]. 


\subsection{Main results}

Composition theorems for Fourier integral operators have already been understood in many cases. The analogous question for the composition of Fourier integral operators and paired Lagrangian distributions has not been studied systematically. In the particular case when the Fourier integral operators are associated with a symplectomorphism, one has already a positive answer. Namely, let $F$ be a classical Fourier integral operator of order $r$ which associates with a symplectomorphism mapping the pair $\left(\Lambda_{0}, \Lambda_{1}\right)$ to $\left(\Lambda_{0}^{\prime}, \Lambda_{1}^{\prime}\right)$. Then $F: I^{m, p}\left(\Lambda_{0}, \Lambda_{1}\right) \rightarrow I^{m+r, p+r}\left(\Lambda_{0}^{\prime}, \Lambda_{1}^{\prime}\right)$ []os98, Theorem 7.4]. Later in [Jos99], Joshi proposed and proved a part of a composition theorem under a more general condition. The order and principal symbol of the composite was, however, not given.

In the same situation, the composition theorem for paired Lagrangian operators has been proved only in a particular case. More precisely, only the case of paired Lagrangian distributions when the first canonical relation is the diagonal and the second one is a flow-out manifold, i.e., the one obtained from the flow of the characteristic set under a bicharacteristic flow. For details, see [AU85, Theorem 0.1], and [Jos98, Theorem 11.2]. More general cases of canonical relations were unknown.

My dissertation is to study composition theorems for paired Lagrangian distributions in general, under some geometric conditions on the canonical relations. The first main result is a new characterisation of paired Lagrangian distributions via multi-phase functions relying on work by Mendoza [Men82], which is an extension of Hörmander's representation of Lagrangian distributions in terms of oscillatory integrals, see Chapter $\mathbb{W}$. As an important consequence, we get a second result on the strong composition of Fourier integral operators and paired Lagrangian distributions, under a certain condition on the canonical relations, see Theorem 2.8 . Note that when this condition fails, in general, the composite is not a paired Lagrangian distribution, see Theorem 2.46 and Corollary 2.49.

The compositions of paired Lagrangian distributions are highly nontrivial. Under the natural condition of clean composition above, the wave front relation of the composite is contained in four Lagrangian manifolds. This fact suggests that composition of two paired Lagrangian distributions belongs to some class of distributions associated with a system of $\kappa$ Lagrangian manifolds, where $\kappa \in\{1,2,3,4\}$.

The first difficulty is that such spaces of distributions have not been investigated yet; only few results in particular case were known. We are the first to investigate such spaces of distributions related to a clean system of $\kappa$ Lagrangian manifolds in full generality, 
where again $\kappa \in\{1,2,3,4\}$. The third result in my research is to give the oscillatory representation for a class of distributions which is associated with a certain system of $\kappa$ Lagrangian manifolds, for $\kappa=3$ and $\kappa=4$, and to prove that under a suitable condition, the composed operator of two paired Lagrangian distributions has Schwartz kernel belonging to such class of distributions defined by $\kappa$ Lagrangian manifolds.

The class of properly supported Fourier integral operators is closed under clean, proper, connected composition [Hör85, Theorem 25.2.3]. A natural question for the class of properly supported paired Lagrangian distribution arises. More precisely, let $\left(C_{0}, C_{1}\right)$ and $\left(\tilde{C}_{0}, \tilde{C}_{1}\right)$ be cleanly intersecting pairs of homogeneous canonical relations from $T^{*} Y \backslash 0$ to $T^{*} X \backslash 0$, and from $T^{*} Z \backslash 0$ to $T^{*} Y \backslash 0$, respectively. Find a condition on $\left(C_{0}, C_{1}\right)$ and $\left(\tilde{C}_{0}, \tilde{C}_{1}\right)$ such that for any properly operators $A \in I^{m_{0}, m_{1}}\left(X, Y ; C_{0}, C_{1}\right), B \in I^{p_{0}, p_{1}}\left(Y, Z ; \tilde{C}_{0}, \tilde{C}_{1}\right)$, the composed operator $A \circ B$ is a paired Lagrangian distribution. One of our new results is to establish such a condition, see Corollary [2.38.

In another direction, new wave front relations of the composed operators arise automatically when zero sections are allowed, that is, in the definition of the canonical relation above we replace $\left(T^{*} X \backslash 0\right) \times\left(T^{*} Y \backslash 0\right)$ by $T^{*} X \times\left(T^{*} Y \backslash 0\right)$ or by $\left(T^{*} X \backslash 0\right) \times T^{*} Y$. For example, suppose that $C_{1}, C_{2}$ satisfy all conditions of [Hör85], Theorem 25.2.3], except that $C_{2} \subset\left(T^{*} Y \backslash 0\right) \times\left(T^{*} Z \backslash 0\right)$ is replaced by $C_{2} \subset T^{*} Y \times\left(T^{*} Z \backslash 0\right)$. Then Hörmander's calculus of wave front sets yields $\mathrm{WF}^{\prime}(A \circ B) \subseteq\left(C_{1} \circ C_{2}\right) \cup\left(0_{X \times Y} \circ C_{2}\right)$, where $0_{X \times Z}$ is the zero section of $T^{*} Y \times T^{*} Z$. This extra term shows that, in general, $A \circ B$ is not a Fourier integral operator. Actually, we prove that the composite is a paired Lagrangian distribution (see Proposition 2.10 for details).

Finally, some models for strong compositions with or without CIS condition are given at the end of Chapter 2 . 


\section{Paired Lagrangian distributions}

In this chapter, we first recall the standard objects in the theory of Fourier integral operators, for example, phase functions, conic Lagrangian submanifolds and theory of composition of canonical relations. Our aim is to use multiphase functions to parametrize pair of Lagrangian submanifolds, and then to define the class of distributions corresponding to these multiphase functions via oscillatory integrals. The main result in this chapter is the new characterisation of paired Lagrangian distributions by oscillatory integral of multi-phase functions and amplitude functions. One advantage of this approach is to make paired Lagrangian distributions be a natural generalization of Lagrangian distributions. This plays an essential role in composition theory for paired Lagrangian distributions. We shall briefly recall some results in Lagrangian distributions and composition of Fourier integral operators. For details, see [Hör85], [BG81].

\subsection{Well-known results about Fourier integral operators}

\subsubsection{Basic definitions}

Let $X$ be a $C^{\infty}$ real manifold of dimension $n$ with local coordinates $x_{1}, \ldots x_{n}$. Then the cotangent bundle $T^{*} X$ is a homogeneous symplectic manifold with respect to the multiplication on the fibre and the canonical symplectic form $\sigma=d \xi \wedge d x$, where $\xi$ is the dual coordinates.

A subset $\Lambda$ of $T^{*} X \backslash 0$ is called a conic Lagrangian submanifold of $T^{*} X$ if $\Lambda$ is a conic $n$-submanifold and $\left.\sigma\right|_{\Lambda}=0$. A submanifold $\Sigma$ of $T^{*} X$ is called an isotropic manifold if its tangent space at each point is an isotropic vector subspace.

Definition 1.1. Let $Y, Z$ be submanifolds of a smooth manifold $M$. We say that $Y$ intersects $Z$ cleanly if $Y \cap Z$ is a submanifold and for all $p \in Y \cap Z$, we have $T_{p}(Y \cap Z)=T_{p} Y \cap T_{p} Z$. 
The non-negative integer

$$
e=\operatorname{codim} Y+\operatorname{codim} Z-\operatorname{codim}(Y \cap Z)
$$

is called the excess of this clean intersection.

A pair $\left(\Lambda_{0}, \Lambda_{1}\right)$ is called a Lagrangian pair if $\Lambda_{0}, \Lambda_{1}$ are closed conic Lagrangian manifolds of $T^{*} X \backslash 0$ and furthermore, $\Lambda_{0}$ and $\Lambda_{1}$ intersect cleanly.

Note that if a Lagrangian pair has excess $e$ then its intersection is a $e$-dimensional submanifold.

Definition 1.2. A relation $C$ from $T^{*} Y \backslash 0$ to $T^{*} X \backslash 0$ is called a canonical relation if its twisted defined by

$$
C^{\prime}=\left\{(x, y, \xi, \eta) \in T^{*}(X \times Y) \mid(x, \xi ; y,-\eta) \in C\right\}
$$

is a closed conic Lagrangian manifold in $T^{*}(X \times Y) \backslash 0$.

The geometric idea staying behind phase functions is the composition of canonical relations.

\subsubsection{Composition of canonical relations}

We recall the composition of canonical relation in general setting:

Theorem 1.3 ([[Hör85]). Let $S_{j}$ be a symplectic manifold with symplectic form $\sigma_{j}, j=$ 1,2,3. If $G_{1} \subset S_{1} \times S_{2}$ and $G_{2} \subset S_{2} \times S_{3}$ are Lagrangians for the symplectic form $\sigma_{1}-\sigma_{2}$ and $\sigma_{2}-\sigma_{3}$, and $G_{1} \times G_{2}$ intersects $S_{1} \times \Delta_{S_{2}} \times S_{3}$ cleanly in $G$ with excess $e$, then the projection $\pi$ from $G$ to $S_{1} \times S_{3}$ has rank $\left(\operatorname{dim} S_{1}+\operatorname{dim} S_{3}\right) / 2$, and the range

$$
G_{1} \circ G_{2}=\left\{\left(\gamma_{1}, \gamma_{3}\right) \mid \exists \gamma_{2} \in S_{2}:\left(\gamma_{1}, \gamma_{2}\right) \in G_{1},\left(\gamma_{2}, \gamma_{3}\right) \in G_{2}\right\}
$$

of $\pi$ is locally a Lagrangian manifold with respect to $\sigma_{1}-\sigma_{3}$. Under these hypotheses we shall say that the composition is clean and the number $e$ is called the excess of the clean composition.

Note that the map $\pi$ has $e$-dimensional fibre. The excess can be computed by the formula $\operatorname{dim}\left(G_{1} \times G_{2}\right) \cap\left(S_{1} \times \Delta_{S_{2}} \times S_{3}\right)-\left(\operatorname{dim} S_{1}+\operatorname{dim} S_{3}\right) / 2$. Similarly, the compositions of isotropic canonical relations are stated in the following theorem. 
Theorem 1.4 ([ $[\mathrm{BG} 81]])$. Let $X, Y$ be manifolds, and $\Gamma$ and $\Sigma$ be closed conic isotropic submanifolds of $T^{*}(X \times Y) \backslash 0$ and $T^{*} Y \backslash 0$ respectively, which satisfy the following conditions:

a) $\Gamma \cap\left(T^{*} X \times 0_{Y}\right)=\emptyset$.

b) $\Gamma \circ \Sigma \subset T^{*} X \backslash 0$.

c) Let $\Gamma^{\prime}$ be the projection of $\Gamma$ in $X \times Y$. Then the projection $\Gamma^{\prime} \rightarrow X$ is proper.

d) $\Gamma \times \Sigma$ intersects $T^{*} X \times \Delta_{T^{*} Y}$ cleanly.

e) The map $\tau:(\Gamma \times \Sigma) \cap T^{*} X \times \Delta_{T^{*} Y} \rightarrow \Gamma \rightarrow T^{*} X$ is of constant rank.

Then $\Gamma \circ \Sigma$ is an (immersed) isotropic submanifold of $T^{*} X \backslash 0$ and the map is a fibre mapping with compact fibre of dimension $\tilde{e}$. The excess of the clean intersection will be called the excess of this composition.

Remark 1.5. - For all $p \in(\Gamma \times \Sigma) \cap\left(T^{*} X \times \Delta_{T^{*} Y}\right)$, let $L=T_{p}(\Gamma \times \Sigma), \Delta:=T_{p}\left(T^{*} X \times \Delta_{T^{*} Y}\right)$. Then

$$
\begin{gathered}
T_{\tau(p)}(\Gamma \circ \Sigma) \simeq \frac{L \cap \Delta}{L \cap \Delta^{\sigma}}, \\
T_{\tau(p)}(\Gamma \circ \Sigma)^{\sigma} / T_{\tau(p)}(\Gamma \circ \Sigma) \simeq \frac{L^{\sigma} \cap \Delta^{\sigma}}{L \cap \Delta^{\sigma}} \\
\operatorname{dim} X-\operatorname{dim} \Gamma \circ \Sigma=\operatorname{dim} Y-\operatorname{dim} \Sigma-\operatorname{dim} \frac{L^{\sigma} \cap \Delta}{L \cap \Delta^{\sigma}},
\end{gathered}
$$

where $\sigma$ is the standard symplectic structure of $(X \times Y) \times Y$.

So the excess of the clean intersection is

$$
\begin{aligned}
e=\operatorname{dim}(L+\Delta)^{\sigma} & =\operatorname{dim} L^{\sigma}+\operatorname{dim} \Delta^{\sigma}-\operatorname{dim}(L \cap \Delta)^{\sigma} \\
& =\operatorname{dim}(L \cap \Delta)+2 \operatorname{dim} Y-\operatorname{dim} L \\
& =\operatorname{dim}(L \cap \Delta)+2 \operatorname{dim} Y-\operatorname{dim} \Gamma-\operatorname{dim} \Sigma \\
& =(\operatorname{dim} \Gamma \circ \Sigma-(\operatorname{dim} X+\operatorname{dim} Y-\operatorname{dim} \Sigma) .
\end{aligned}
$$

The compact fibre of the map $\tau$ has the dimension

$$
\tilde{e}=\operatorname{dim} L \cap \Delta^{\sigma} .
$$

In general we have $e \geq \tilde{e}$. The equality occurs if $\operatorname{dim} L=\operatorname{dim} L^{\sigma}$, for instance, when $\Sigma$ is a Lagrangian submanifold, the condition e) in this theorem holds automatically. Hence, this condition disappears in the composition of canonical relations. 
- In the case $\Sigma$ is the intersection of a cleanly intersecting pair of Lagrangian submanifolds, namely $\left(\Lambda_{0}, \Lambda_{1}\right)$, then the condition $e$ ) is equivalent to $\left(L_{0}(p) \cap L_{1}(p)\right) \cap \Delta(p)^{\sigma}$ (or $\left.L_{0}(p)+L_{1}(p)+\Delta(p)\right)$ which has constant dimension, where $L_{j}(p)=T_{p}\left(\Gamma \times \Lambda_{j}\right)$. It holds in particular when $\left(L_{0}+L_{1}\right) \cap \Delta=\left(L_{0} \cap \Delta\right)+\left(L_{1} \cap \Delta\right)$ and $L_{j} \cap \Delta, L_{0} \cap L_{1} \cap \Delta$ have constant dimensions.

Now we introduce a criterion which preserves the cleanness of Lagrangian pair under composition.

Definition 1.6 (Joshi). Three smooth submanifolds $X, Y, Z$ of a smooth manifold $M$ are called an intersecting triple if all the pairwise intersections are cleanly intersecting pairs, the pairwise intersections of the pairwise intersections and the original manifolds are clean, and for all $p \in X \cap Y \cap Z$,

$$
T_{p} X \bigcap\left(T_{p} Y+T_{p} Z\right)=\left(T_{p} X \bigcap T_{p} Y\right)+\left(T_{p} X \bigcap T_{p} Z\right) .
$$

The condition (IL.4) is equivalent to the following dimensional relation:

$$
\begin{aligned}
\operatorname{dim}\left(T_{p} X+T_{p} Y+T_{p} Z\right)=[\operatorname{dim} X+\operatorname{dim} Y+\operatorname{dim} Z & -\operatorname{dim}(X \cap Y)-\operatorname{dim}(Y \cap Z) \\
& -\operatorname{dim}(Z \cap X)+\operatorname{dim}(X \cap Y \cap Z)] .
\end{aligned}
$$

We recall a geometric part for composition of Fourier integral operators and paired Lagrangian distributions:

Theorem 1.7 ([]0s99]). Let X, Y,Z be smooth manifolds, $\Lambda$ be a Lagrangian submanifold in $T^{*}(X \times Y)$ and $\left(\Lambda_{0}, \Lambda_{1}\right)$ be a cleanly intersection pair of Lagrangian submanifolds in $T^{*}(Y \times Z)$. If $\left(\Lambda \times \Lambda_{0}, \Lambda \times \Lambda_{1}, T^{*} X \times N^{*}\left(\Delta_{Y}\right) \times T^{*} Z\right)$ is an intersecting triple, then locally $\left(\Lambda \circ \Lambda_{0}, \Lambda \circ \Lambda_{1}\right)$ is a cleanly intersecting pair of Lagrangian submanifolds.

Proof. Set $\Sigma=\Lambda_{0} \cap \Lambda_{1}$. First, we shall show that $\Lambda \circ \Lambda_{j}$ and $\Lambda \circ \Sigma$ are smooth isotropic manifolds. Since $\Lambda \times \Lambda_{0}, \Lambda \times \Lambda_{1}$ intersect $\left.T^{*} X \times N^{*}\left(\Delta_{Y}\right) \times T^{*} Z\right)$, we have $\Lambda \circ \Lambda_{0}, \Lambda \circ \Lambda_{1}$ are Lagrangian submanifolds in $T^{*} X$. Moreover, $\Lambda \times \Sigma=\cap_{j=1,2} \Lambda \times \Lambda_{j}$ intersects $T^{*} X \times$ $N^{*}\left(\Delta_{Y}\right) \times T^{*} Z$ cleanly. However, this condition solely is not enough to conclude that $\Lambda \circ \Sigma$ is an isotropic submanifold in $T^{*} X$. Indeed, the tangent condition (IL.4) implies fulfillment of condition $e$ ) in Theorem L.4. Therefore, $\Lambda \circ \Sigma$ is an isotropic manifold in $T^{*} X$.

Now we shall show that $T_{p}(\Lambda \circ \Sigma)=\cap_{j=0,1} T_{p}\left(\Lambda \circ \Lambda_{j}\right)$ for all $p \in \Lambda \circ \Sigma$. For brevity, denote $L_{j}:=T_{p}\left(\Lambda \times \Lambda_{j}\right), \Delta:=T_{p}\left(T^{*} X \times N^{*}\left(\Delta_{Y}\right) \times T^{*} Z\right)$, and $\sigma$ as the standard symplectic form in the tangent space $X \times Y^{-} \times\left(Y \times Z^{-}\right)$. The condition (‥4) can be written as:

$$
\left(L_{0}+L_{1}\right) \cap \Delta=\left(L_{0} \cap \Delta\right)+\left(L_{1} \cap \Delta\right) .
$$


Since $L_{0}, L_{1}$ are Lagrangian subspaces, taking the orthogonal complements with respect to $\sigma$, we obtain

$$
\left(L_{0} \cap L_{1}\right)+\Delta^{\sigma}=\left(L_{0}+\Delta^{\sigma}\right) \cap\left(L_{1}+\Delta^{\sigma}\right) .
$$

Using the fact that $\Delta$ is an co-isotropic subspace, we obtain

$$
\left(L_{0} \cap L_{1} \cap \Delta\right)+\Delta^{\sigma}=\left(\left(L_{0} \cap \Delta\right)+\Delta^{\sigma}\right) \cap\left(\left(L_{1} \cap \Delta\right)+\Delta^{\sigma}\right)
$$

This means that

$$
\frac{L_{0} \cap L_{1} \cap \Delta}{L_{0} \cap L_{1} \cap \Delta^{\sigma}}=\frac{L_{0} \cap \Delta}{L_{0} \cap \Delta^{\sigma}} \cap \frac{L_{1} \cap \Delta}{L_{1} \cap \Delta^{\sigma}} .
$$

Identifying $\frac{L_{0} \cap L_{1} \cap \Delta}{L_{0} \cap L_{1} \cap \Delta^{\sigma}}$ with $T_{p}(\Gamma \circ \Sigma)$ and $\frac{L_{0} \cap \Delta}{L_{0} \cap \Delta^{\sigma}} \cap \frac{L_{1} \cap \Delta}{L_{0} \cap \Delta^{\sigma}}$ with $T_{p}\left(\Lambda \circ \Lambda_{0}\right) \cap T_{p}\left(\Lambda \circ \Lambda_{1}\right)$, we conclude that $\left(\Lambda \circ \Lambda_{0}\right.$ and $\left.\Lambda \circ \Lambda_{1}\right)$ intersect cleanly.

Remark 1.8. This theorem can be formulated in the language of the composition theory. Indeed, let $\left(\Lambda \times \Lambda_{0}, \Lambda \times \Lambda_{1}, T^{*} X \times \Delta_{T^{*} Y} \times T^{*} Z\right)$ be an intersecting triple. Then all geometric informations are decoded in the following:

- The set $\Sigma=\Lambda_{0} \cap \Lambda_{1}$ is an $l$-codimensional submanifold of $\Lambda_{0}$, where the number $l$ can be computed as $l=2 \operatorname{dim} X+2 \operatorname{dim} Y-\operatorname{dim}\left(\Lambda \times \Lambda_{0} \cap \Lambda \times \Lambda_{1}\right)$.

- The composition $\Lambda \circ \Lambda_{j}, j=0,1$, is clean with excess $e_{j}=\operatorname{dim} L_{j} \cap \Delta^{\sigma}$.

- The composition $\Lambda \circ \Sigma$ is clean with excess $e=\operatorname{dim}\left(\left(L_{0}+L_{1}\right) \cap \Delta^{\sigma}\right)$.

- The set $\Lambda \circ \Sigma$ is an isotropic submanifold of dimension $(\operatorname{dim} X+\operatorname{dim} Z)+l^{\prime}$.

- Then the tangent condition is equivalent to

$$
l+e_{0}+e_{1}-2 e-l^{\prime}=0
$$

As a corollary, we see that $l^{\prime} \leq l$. The equality occurs if and only if $e_{1}=e_{0}=e$. If $e_{0}=0$, then $e=e_{1}$ and $l^{\prime}=l-e_{1}$.

\subsubsection{Phase functions}

Suppose that $\phi: X \times\left(\mathbb{R}^{N} \backslash 0\right) \rightarrow \mathbb{R}$ is a real homogeneous function of degree 1 . Define $G=\left\{\left(x, \phi_{x} ; \theta,-\phi_{\theta}\right):(x, \eta) \in X \times \mathbb{R}^{N}\right\}$ as the canonical relation from $T^{*}\left(\mathbb{R}^{N}\right)$ to $T^{*} X \backslash 0$ generated by $\phi$. Let $G_{0}=\mathbb{R}^{N} \times 0$ be the zero section of $T^{*}\left(\mathbb{R}^{N}\right)$. By composition theory of canonical relations, $\Lambda_{0}=G \circ G_{0}$ is a Lagrangian manifold if $G \times G_{0}$ intersects $T^{*} X \times \Delta_{T^{*}\left(\mathbb{R}^{N}\right)}$ 
cleanly. As we will see, this intersection is clean if and only if $d \phi_{\theta}$ has constant rank on the critical set $C_{\phi}=\left\{(x, \theta) \mid \phi_{\theta}=0\right\}$. By a simple calculation, we will show that $G \circ G_{0}=\left\{\left(x, \phi_{x}\right) \mid(x, \theta) \in C_{\phi}\right\}$, which leads to the definition of a clean phase function that parametrizes a Lagrangian manifold:

Definition 1.9. Let $\Gamma$ be a conic subset of $X \times\left(\mathbb{R}^{N} \backslash 0\right)$ and $\phi \in C^{\infty}(\Gamma)$ be a real valued function which is homogeneous of degree 1 . The function $\phi$ is called a local phase function of $X$ with excess $e$ if the following conditions hold

- $d_{x, \theta} \phi(x, \theta)$ is non-zero in $\Gamma$.

- On the critical set $C_{\phi}=\left\{(x, \theta) \in \Gamma \mid \phi_{\theta}(x, \theta)=0\right\}, d \phi_{\theta}$ has constant rank $N-e$.

If $\phi$ is a local phase function, then $\Lambda_{\phi}:=\left\{\left(x, \varphi_{x}(x, \theta)\right) \mid(x, \theta) \in C_{\phi}\right\}$ is a Lagrangian submanifold. If $\Lambda_{\phi}$ is an embedded submanifold, then $\phi$ is a phase function.

Let $X \subseteq \mathbb{R}^{n}, \Gamma, \tilde{\Gamma}$ be open conic sets in $X \times \mathbb{R}^{N}, X \times \mathbb{R}^{\tilde{N}}$ and $\phi, \tilde{\phi}$ be phase functions in $\Gamma, \tilde{\Gamma}$ respectively.

Definition 1.10. Two phase functions $\phi, \tilde{\phi}$ are equivalent if there exists a fibre preserving diffeomorphism

$$
\Gamma \ni(x, \theta) \mapsto(x, \tilde{\theta}(x, \theta)) \in \tilde{\Gamma}
$$

where $\tilde{\theta}$ is $C^{\infty}$ homogeneous of degree 1 in $\theta$ such that $\phi(x, \theta)=\tilde{\phi}(x, \tilde{\theta}(x, \theta))$.

Note that two equivalent phase functions have the same excess, the same number of fibre and parametrize the same Lagrangian submanifold. We have a criterion for the equivalence of phase functions:

Theorem 1.11 ([[Hör71], [Tre80]). Let $\phi$ and $\tilde{\phi}$ be clean phase functions in conic neighbourhoods of $\left(x_{0}, \theta_{0}\right) \in X \times\left(\mathbb{R}^{N} \backslash 0\right)$ and $\left(x_{0}, \tilde{\theta}_{0}\right) \in X \times\left(\mathbb{R}^{N} \backslash 0\right)$, respectively. Then the functions $\phi$ and $\tilde{\phi}$ are equivalent in some conic neighbourhoods of these points under a diffeomorphism mapping $\left(x_{0}, \theta_{0}\right)$ to $\left(x_{0}, \tilde{\theta}_{0}\right)$ if and only if

i) Two functions $\phi$ and $\tilde{\phi}$ parametrize the same Lagrangian submanifold near $\left(x_{0}, \theta_{0}\right)$ and $\left(x_{0}, \tilde{\theta}_{0}\right)$.

ii) $N=\tilde{N}, e=\tilde{e}$.

iii) Two matrices $\phi_{\theta \theta}\left(x_{0}, \theta_{0}\right)$ and $\phi_{\tilde{\theta} \tilde{\theta}}\left(x_{0}, \theta_{0}\right)$ have the same signature. 
Now we recall some basic transforms on the class of phase function: adding the number of fibre variables, reducing the number of fibre variables and eliminating the excess.

Firstly, if $\phi(x, \theta)$ is a phase function in some conic neighbourhood in $X \times\left(\mathbb{R}^{N} \backslash 0\right)$ of $\left(x_{0}, \theta_{0}\right)$, we can always increase the number of fibres as many as we need. In fact, consider a new function

$$
\psi(x, \theta, \eta)=\phi(x, \theta)+Q(\eta) /|\theta|
$$

where $Q$ is a non-degenerate real quadratic form on $\mathbb{R}^{r}$. This function is a phase function in some conic neighbourhood in $X \times \mathbb{R}^{N+r}$ of the point $\left(x_{0}, \theta_{0}, 0\right)$. Note that $\psi$ and $\phi$ parametrize the same Lagrangian submanifold near $\left(x_{0}, \theta_{0}\right)$ and $\left(x_{0}, \theta_{0}, 0\right)$.

Secondly, let $\phi(x, \theta)$ be a clean phase function in a conic neighbourhood of $\left(x_{0}, \theta_{0}\right) \in$ $C_{\phi}$. If the matrix $\phi_{\theta \theta}\left(x_{0}, \theta_{0}\right)$ has the rank at least $r$, then we can reduce the fibre dimension by $r$. Namely, rewrite $\theta=\left(\theta^{\prime}, \theta^{\prime \prime}\right) \in \mathbb{R}^{N-r} \times \mathbb{R}^{r}$ such that $\phi_{\theta^{\prime \prime} \theta^{\prime \prime}}\left(x_{0}, \theta_{0}\right)$ is invertible. Note that $\theta_{0}^{\prime} \neq 0$. By the implicit function theorem, the equation $\phi_{\theta^{\prime \prime}}=0$ is solved by $\theta^{\prime \prime}=g\left(x, \theta^{\prime}\right)$ near $\left(x_{0}, \theta_{0}\right)$. Consider the change of variables $(x, \theta) \mapsto\left(x, \eta^{\prime}, \eta^{\prime \prime}+g\left(x, \eta^{\prime}\right)\right)$. The new phase function $\tilde{\phi}(x, \theta)=\phi\left(x, \theta^{\prime}, \theta^{\prime \prime}+g\left(x, \eta^{\prime}\right)\right)$ is equivalent to $\phi$ and has the property that $\tilde{\phi}_{\eta^{\prime \prime}}=0$ if and only if $\theta^{\prime \prime}=0$. So we put this assumption for $\phi$. Using Taylor's formula, we have

$$
\phi\left(x, \theta^{\prime}, \theta^{\prime \prime}\right)=\phi\left(x, \eta^{\prime}, 0\right)+\left\langle B(x, \theta) \theta^{\prime \prime}, \theta^{\prime \prime}\right\rangle / 2
$$

where $B$ is a symmetric matrix. Denote $Q\left(x, \theta^{\prime}\right)=\phi_{\theta^{\prime \prime} \theta^{\prime \prime}}\left(x, \theta^{\prime}, 0\right)$. By Morse's lemma, we prove that there exists a change of coordinates

$$
(x, \theta) \mapsto\left(x, \theta^{\prime}, R(x, \theta) \theta^{\prime \prime}\right),
$$

where $R$ satisfies the matrix equation $R^{\prime}(x, \theta) Q\left(x, \theta^{\prime}\right) R(x, \theta)=\left|\theta^{\prime}\right| B(x, \theta)$. So we conclude that in the new coordinates

$$
\phi(x, \theta)=\psi\left(x, \theta^{\prime}\right)+\left\langle Q\left(x, \theta^{\prime}\right) \theta^{\prime \prime}, \theta^{\prime \prime}\right\rangle / 2\left|\theta^{\prime}\right|,
$$

where $\psi\left(x, \theta^{\prime}\right)=\phi\left(x, \theta^{\prime}, 0\right)$ is the clean phase function with the same excess which parametrizes the same Lagrangian submanifold as $\phi . Q$ is a non-singular symmetric matrix whose entries are homogeneous of degree 0.

Thirdly, we now describe the procedure for eliminating the excess of a clean phase function. Roughly speaking, for each clean phase function $\phi$ with excess $e$, there is a splitting $\theta=\left(\theta^{\prime}, \theta^{\prime \prime}\right) \in \mathbb{R}^{N-e} \times \mathbb{R}^{e}$ such that $\phi_{\eta^{\prime \prime}}(x, \eta)=0$. In fact, since $\phi$ has excess $e$, we can rewrite $\theta=\left(\theta^{\prime}, \theta^{\prime \prime}\right) \in \mathbb{R}^{N-e} \times \mathbb{R}^{e}$ such that $d \phi_{\theta^{\prime}}$ has rank $N-e$. We claim $\theta_{0}^{\prime} \neq 0$ because 
otherwise, by Euler's homogeneity relation $\phi_{x}\left(x_{0}, \theta_{0}\right)=\phi_{x \theta^{\prime}}\left(x_{0}, \theta_{0}\right) \theta_{0}^{\prime}+\phi_{x \theta^{\prime \prime}}\left(x_{0}, \theta_{0}\right) \theta_{0}^{\prime \prime}=0$, which contradicts to the fact that $\phi$ is a phase function. Since the submanifold $C_{\phi}$ is defined by $N-e$ equations $\phi_{\theta^{\prime}}=0$ and $\phi_{\theta}^{\prime \prime}=0$ on this set, there exists a matrix $a(x, \theta)$ satisfying $\phi_{\theta^{\prime \prime}}(x, \theta)=\phi_{\theta^{\prime \prime}}(x, \theta) a(x, \theta)$. Consider a change of coordinates of the form

$$
(x, \theta) \mapsto\left(x, \theta^{\prime}+\tilde{a}(x, \theta), \theta^{\prime \prime}\right),
$$

where $\tilde{a}$ is determined via the ODE system

$$
\frac{\partial \tilde{a}(x, \theta)}{\partial \theta^{\prime \prime}}=a(x, \theta), \tilde{a}\left(x, \theta^{\prime}, 0\right)=0 .
$$

In the new coordinates let $\tilde{\phi}(x, \theta)=\phi\left(x, \theta^{\prime}+\tilde{a}(x, \theta), \theta^{\prime \prime}\right)$. Then $\tilde{\phi}$ is an equivalent phase function for $\phi$ satisfying $\tilde{\phi}_{\theta^{\prime \prime}}(x, \theta)=0$.

Remark 1.12. The change of phase functions plays an important role in the investigation of not only the global definition of Lagrangian distribution but also its principal symbol. A remarkable property is that the classes of phase functions and amplitude functions are invariant under action of fibre preserving diffeomorphisms.

\subsubsection{Spaces of amplitude functions}

Let assume that $X$ is an open subset of $\mathbb{R}^{n}$ and $m \in \mathbb{R}$.

Definition 1.13. We will denote by $S^{m}\left(X \times \mathbb{R}^{N}\right)$ the space of all $a \in C^{\infty}\left(X \times \mathbb{R}^{N}\right)$ such that for all compact subset $K \Subset X$ and all multi-indices $\alpha \in \mathbb{N}^{n}, \beta \in \mathbb{N}^{N}$, there exists a constant $C(K, \alpha, \beta)$ such that

$$
\left|\partial_{x}^{\alpha} \partial_{\eta}^{\beta} a(x, \theta)\right| \leq C\langle\theta\rangle^{m-|\beta|},(x, \theta) \in K \times \mathbb{R}^{N},
$$

where $\langle\theta\rangle=\left(1+|\theta|^{2}\right)^{1 / 2}$. We call $S^{m}\left(X \times \mathbb{R}^{N}\right)$ the space of symbols of order $m$.

Note that $S^{m}\left(X \times \mathbb{R}^{N}\right)$ is a Fréchet space with the semi-norms

$$
p_{K, \alpha, \beta}(u)=\sup _{(x, \theta) \in K \times \mathbb{R}^{N}}\left|\partial_{x}^{\alpha} \partial_{\eta}^{\beta} a(x, \theta)\right|\langle\theta\rangle^{-m+|\beta|}
$$

for $K$ compact in $X$. Let

$$
S^{-\infty}\left(X \times \mathbb{R}^{N}\right)=\cap_{m \in \mathbb{R}} S^{m}\left(X \times \mathbb{R}^{N}\right), \quad S^{\infty}\left(X \times \mathbb{R}^{N}\right)=\cup_{m \in \mathbb{R}} S^{m}\left(X \times \mathbb{R}^{N}\right) .
$$

We mention some important properties of these symbol spaces 
- For all $m^{\prime}>m$ we have $S^{m}\left(X \times \mathbb{R}^{N}\right) \subset S^{m^{\prime}}\left(X \times \mathbb{R}^{N}\right)$.

- If $m^{\prime}>m$ then $S^{-\infty}\left(X \times \mathbb{R}^{N}\right)$ is dense in $S^{m}\left(X \times \mathbb{R}^{N}\right)$ with respect to the topology of $S^{m^{\prime}}\left(X \times \mathbb{R}^{N}\right)$.

- Let $a_{j} \in S^{m_{j}}\left(X \times \mathbb{R}^{N}\right), j=0,1, \ldots$, with $m_{j} \rightarrow-\infty$. Then there exists a unique $a \in S^{m}\left(X \times \mathbb{R}^{N}\right)$ modulo $S^{-\infty}\left(X \times \mathbb{R}^{N}\right)$, such that $\left(a-\sum_{j=0}^{k-1} a_{j}\right) \in S^{m_{k}}\left(X \times \mathbb{R}^{N}\right)$ for all $k \in \mathbb{N}$. We call $a$ the asymptotic sum of $a_{j}$ and write $a \sim \sum a_{j}$.

In application, the following criterion is very useful.

Proposition 1.14. Let a $a_{j}$ belong to $S^{m_{j}}\left(X \times \mathbb{R}^{N}\right), m_{j} \rightarrow-\infty$, and $a \in C^{\infty}\left(X \times \mathbb{R}^{N}\right)$ satisfying: a) For all compact $K \Subset X, \alpha \in \mathbb{N}^{n}, \beta \in \mathbb{N}^{N}$, there exists a constant $M_{\alpha, \beta}>0$ such that

$$
\left|\partial_{x}^{\alpha} \partial_{\eta}^{\beta} a(x, \theta)\right| \leq C\langle\theta\rangle^{m-|\beta|},(x, \theta) \in K \times \mathbb{R}^{N} .
$$

b) There exists a sequence $m_{k}^{\prime} \rightarrow-\infty$ as $k \rightarrow \infty$ such that for all compact $K \Subset X$ and $k \in \mathbb{N}$

$$
\left|a(x, \theta)-\sum_{j=0}^{k-1} a_{j}(x, \theta)\right| \leq C\langle\theta\rangle^{m_{k}^{\prime}}, \quad(x, \theta) \in K \times \mathbb{R}^{N},
$$

for some constant $C$.

Then $a \sim \sum a_{j}$.

\subsubsection{Local representation of Lagrangian distributions}

Let $\phi(x, \theta)$ be a phase function and $a \in S^{m}\left(X \times \mathbb{R}^{N}\right), m<-k-N, k \in \mathbb{N}$. Then

$$
I(a, \phi):=\int e^{i \phi(x, \theta)} a(x, \theta) d \theta \in C^{k}(X) .
$$

Moreover, the map $a \mapsto I(a, \phi)$ is continuous from $S^{m}\left(X \times \mathbb{R}^{N}\right)$ to $C^{k}(X)$. In general, using oscillatory integral, we obtain the following result:

Theorem 1.15 ([[Hör85]). Let $\phi(x, \theta)$ be a phase function on $X \times\left(\mathbb{R}^{N} \backslash 0\right)$. Then there is a unique way to define $I(a, \phi) \in \mathcal{D}^{\prime}(X)$ for $a \in S^{\infty}\left(X \times \mathbb{R}^{N}\right)$ such that $I(a, \phi)$ is defined by ([1.9) when $a \in S^{m}\left(X \times \mathbb{R}^{N}\right), m<-N$, and for all $m \in \mathbb{R}$, the map $S^{m}\left(X \times \mathbb{R}^{N}\right) \ni a \mapsto$ $I(a, \phi) \in \mathcal{D}^{\prime}(X)$ is continuous. Furthermore, $\operatorname{WF}(u) \subseteq \Lambda_{\phi}$. More precisely, we have

$$
\mathrm{WF}(u) \subseteq\left\{\left(x, \phi_{x}(x, \theta)\right) \mid(x, \theta) \in \text { cone supp } a, \phi_{\theta}(x, \theta)=0\right\},
$$

where cone supp $a$ is the smallest closed conic subset of $X \times \mathbb{R}^{N} \backslash 0$ outside of which a is of class $S^{-\infty}$. 
Definition 1.16. Let $\phi$ be a phase function with excess $e$ on a conic neighbourhood $\Gamma$ of $X \times \mathbb{R}^{N} \backslash 0$. We shall denote by $I^{m}\left(X ; \Lambda_{\phi}\right), m \in \mathbb{R}$ the space of all distribution $u \in \mathcal{D}^{\prime}(X)$ such that $u=I(a, \phi)$ with some function $a \in S^{m+n / 4-N / 2-e / 2}\left(X \times \mathbb{R}^{N}\right)$ supported in $\Gamma$. Elements of $I^{m}\left(X ; \Lambda_{\phi}\right)$ are called Lagrangian distributions associated with the phase function $\phi$.

Suppose that $\tilde{\phi}(x, \tilde{\theta})$ is another phase function on some conic neighbourhood $\tilde{\Gamma}$ of $X \times\left(\mathbb{R}^{\tilde{N}} \backslash 0\right)$. A natural question that when the two formulas $I(a, \phi), I(\tilde{a}, \tilde{\phi})$ micro-locally represent the same distribution arises. The necessary condition is $\Lambda_{\phi}=\Lambda_{\tilde{\phi}}$. Combining [Hör71, Theorem 4.2.1] and [DG75, Lemma 7.1], we have

Proposition 1.17. Let $\phi$ and $\tilde{\phi}$ be two phase functions and $\left(x_{0}, \xi_{0}\right) \in \Lambda_{\phi} \cap \Lambda_{\tilde{\phi}}$. If $u \in I^{m}\left(\Lambda_{\phi}\right)$ with $\mathrm{WF}(u)$ in a small conic neighbourhood of $\left(x_{0}, \xi_{0}\right)$, then $u \in I^{m}\left(\Lambda_{\tilde{\phi}}\right)$.

Proof. The idea of the proof is as follows. Firstly, we prove for the case both $\phi$ and $\tilde{\phi}$ are non-degenerate phase functions via reducing the fibre of phase functions and the equivalence of phase functions.

Secondly, when $\phi$ is a clean phase function, by eliminating excess of clean phase functions, there exists a non-degenerate phase function $\psi$ such that $\Lambda_{\phi}=\Lambda_{\psi}$ near $\left(x_{0}, \xi_{0}\right)$ and $u \in I^{m}\left(X ; \Lambda_{\psi}\right)$. Similarly, $I^{m}\left(X ; \Lambda_{\tilde{\phi}}\right)=I^{m}\left(X ; \Lambda_{\tilde{\psi}}\right)$ for some non-degenerate phase function $\psi$ satisfying $\Lambda_{\tilde{\phi}}=\Lambda_{\tilde{\psi}}$. Using the result on the non-degenerate case, we obtain $I^{m}\left(X ; \Lambda_{\psi}\right)=I^{m}\left(X ; \Lambda_{\tilde{\psi}}\right)$. Hence, $u \in I^{m}\left(X ; \Lambda_{\tilde{\phi}}\right)$. This completes the proof.

Remark 1.18. Another way to prove this result is using stationary phase method. More details can be found in [Hör85, Propositions 25.1.5 and 25.1.5'].

\subsubsection{Global definition of Lagrangian distributions}

Definition 1.19. Let $\Lambda \subset T^{*} X \backslash 0$ be a closed conic Lagrangian manifold. We will denote by $I^{m}(X ; \Lambda), m \in \mathbb{R}$, the space of all $u \in \mathcal{D}^{\prime}(X)$ such that

- the wave front set $\operatorname{WF}(u) \subseteq \Lambda$.

- If $\left(x_{0}, \xi_{0}\right) \in \Lambda$ and $\phi(x, \theta)$ is a clean phase function with excess $e$ in a conic neighbourhood of $\left(x_{0}, \theta_{0}\right)$ such that $\Lambda_{\phi}=\Lambda$ near $\left(x_{0}, \xi_{0}\right)$, then there is a function $a \in S^{m+n / 4-N / 2-e / 2}\left(X \times \mathbb{R}^{N}\right)$ which has support in the interior of a sufficiently small conic neighbourhood of $\left(x_{0}, \theta_{0}\right)$ such that $u=I(a, \phi)+v$ and $\left(x_{0}, \xi_{0}\right) \notin \mathrm{WF}(v)$. 


\subsection{Multiphase functions}

\subsubsection{Definition and geometric interpretation}

Assume that $X \subseteq \mathbb{R}^{n}$ is an open subset and $\mathbb{R}^{N}=\mathbb{R}^{k} \times \mathbb{R}^{l}, \theta=(\eta, \tau)$ and $H: \mathbb{R}^{k} \backslash 0 \rightarrow$ $\mathbb{R}^{l}$ is a homogeneous function of degree one. Let $G=\left\{\left(x, \phi_{x}, \eta, \tau,-\phi_{\eta},-\phi_{\tau}\right)\right\}, G_{0}=$ $\{(\eta, \tau, 0,0)\}, G_{1}=N_{\mathbb{R}^{N}}^{*}\left(\operatorname{graph}_{H}\right)$. Obviously, $G$ is a canonical relation from $T^{*} \mathbb{R}^{N}$ to $T^{*} X$ and $\left(G_{0}, G_{1}\right)$ is a cleanly intersecting pair in $T^{*} \mathbb{R}^{N}$. By applying Theorem $\mathbb{L}$.7 we shall determine an explicit condition such that $\left(G \circ G_{0}, G \circ G_{1}\right)$ is a cleanly intersecting pair of Lagrangian submanifods of $T^{*} X$.

Let us define $M=T^{*} X \times \Delta_{T^{*} \mathbb{R}^{k+l}}, M_{0}=G \times G_{0}, M_{1}=G \times G_{1}$. Then

$$
\begin{aligned}
M \cap M_{0} & =\left\{\left(x, \phi_{x}, \eta, \tau,-\phi_{\eta},-\phi_{\tau} ; \eta, \tau, 0,0\right) \mid \phi_{\eta}=0, \phi_{\tau}=0\right\} \\
M \cap M_{1} & =\left\{\left(x, \phi_{x}, \eta, \tau,-\phi_{\eta},-\phi_{\tau} ; \eta, H(\eta), t H^{\prime}(\eta),-t\right) \mid \phi_{\tau}=t, \phi_{\eta}=-t H^{\prime}(\eta)\right\} \\
M_{0} \cap M_{1} & =\left\{\left(x, \phi_{x}, \eta, \tau,-\phi_{\eta},-\phi_{\tau} ; \eta^{\prime}, H\left(\eta^{\prime}\right), 0,0\right)\right\} \\
M \cap M_{0} \cap M_{1} & =\left\{\left(x, \phi_{x}, \eta, \tau,-\phi_{\eta},-\phi_{\tau} ; \eta, H(\eta), 0,0\right) \mid \phi_{\tau}=0, \phi_{\eta}=0, \tau=H(\eta)\right\},
\end{aligned}
$$

where $x, \eta, \tau, t, \phi_{x}, \phi_{\eta}$ and $\phi_{\tau}$ are row vectors, and $H^{\prime}(\eta)=\left(\partial_{\eta_{i}} H_{j}(\eta)\right)$ is an $l \times k$-matrix with $H=\left(H_{1}, \ldots, H_{l}\right)$. We assume that

$$
\operatorname{rank}\left\{d \phi_{\eta}, d \phi_{\tau}\right\}=k+l-e_{0}, e_{0} \in \mathbb{N} \text { at the point } \phi_{\eta}=0, \phi_{\tau}=0
$$

$\operatorname{rank}\left\{d\left(\phi_{\eta}+\phi_{\tau} H^{\prime}(\eta)\right)\right\}=k-e_{1}, e_{1} \in \mathbb{N}$ at the point $\phi_{\eta}+\phi_{\tau} H^{\prime}(\eta)=0, \tau-H(\eta)=0$.

Under these conditions ( $\left.M \cap M_{0}, M \cap M_{1}, M_{0} \cap M_{1}, M \cap M_{0} \cap M_{1}\right)$ are submanifolds.

Lemma 1.20. For every point $p \in M \cap M_{0}$ we have $T_{p} M \cap T_{p} M_{0}=T_{p}\left(M \cap M_{0}\right)$, and this tangent space is determined by

$$
\begin{aligned}
& \phi_{x \eta} \delta_{x}+\phi_{\eta \eta} \delta_{\eta}+\phi_{\tau \eta} \delta_{\tau}=0, \\
& \phi_{x \tau} \delta_{x}+\phi_{\eta \tau} \delta_{\eta}+\phi_{\tau \tau} \delta_{\tau}=0 .
\end{aligned}
$$

Proof. For every $p \in M \cap M_{0}$ we have

$$
T_{p} M_{0}=\left\{\left(x, \phi_{x}, \eta, \tau,-\phi_{\eta},-\phi_{\tau}, \eta^{\prime}, \tau^{\prime}, 0,0 ; \delta_{x}, d\left(\phi_{x}\right), \delta_{\eta}, \delta_{\tau},-d\left(\phi_{\eta}\right),-d\left(\phi_{\tau}\right), \delta_{\eta}^{\prime}, \delta_{\tau}^{\prime}, 0,0\right)\right\},
$$

where $d\left(\phi_{x}\right)=\phi_{x x} \delta_{x}+\phi_{\eta x} \delta_{\eta}+\phi_{\tau x} \delta_{\tau}, d\left(\phi_{\eta}\right)=\phi_{x \eta} \delta_{x}+\phi_{\eta \eta} \delta_{\eta}+\phi_{\tau \eta} \delta_{\tau}$, and $d\left(\phi_{\tau}\right)=\phi_{x \tau} \delta_{x}+$ $\phi_{\eta \tau} \delta_{\eta}+\phi_{\tau \tau} \delta_{\tau}$. 
Therefore, $T_{p} M_{0} \cap T_{p} M$ is defined by the equations

$$
\begin{array}{r}
\eta^{\prime}=\eta, \tau^{\prime}=\tau, \phi_{\eta}=0, \phi_{\tau}=0, \\
d\left(\phi_{\eta}\right)=0, d\left(\phi_{\tau}\right)=0, \delta_{\eta}^{\prime}=\delta_{\eta}, \delta_{\tau}^{\prime}=\delta_{\tau} .
\end{array}
$$

From (․ㅣㅇ) $\left.), T_{p}\left(M \cap M_{0}\right)\right)$ is defined by equation $d \phi_{\eta}=0, d \phi_{\tau}=0$. Hence, $T_{p}(M \cap$ $\left.M_{0}\right)=T_{p} M \cap T_{p} M_{0}$. This proves the lemma.

Denote $H^{\prime}(\eta)^{T}$ is the transpose of the matrix $H^{\prime}(\eta)$. Similarly, we have the following results:

Lemma 1.21. At every point $p \in M \cap M_{1}$ we have $T_{p} M \cap T_{p} M_{1}=T_{p}\left(M \cap M_{1}\right)$. Moreover, this tangent space is determined by the following system:

$$
\begin{aligned}
\phi_{\tau}=t, \phi_{\eta} & =-t H^{\prime}(\eta), \\
\phi_{x \eta} \delta_{x}+\phi_{\eta \eta} \delta_{\eta}+\phi_{\eta \tau} \delta_{\tau} & =-t H^{\prime \prime}(\eta) \delta_{\eta}-H^{\prime}(\eta)^{T} \delta_{t}, \\
\phi_{x \tau} \delta_{x}+\phi_{\eta \tau} \delta_{\eta}+\phi_{\tau \tau} \delta_{\tau} & =\delta_{t}, \\
H^{\prime}(\eta) \delta_{\eta}-\delta_{\tau} & =0
\end{aligned}
$$

where $p=\left(x, \phi_{x}, \eta, H(\eta),-\phi_{\eta},-\phi_{\tau}, \eta, H(\eta), t H^{\prime}(\eta),-t\right),(p, v) \in T_{p} M$ depends on $\delta_{x}, p_{\eta}, \delta_{\tau}, \delta_{t}$ in the form

$$
v=\left(\delta_{x}, d \phi_{x}, \delta_{\eta}, p_{\tau},-d \phi_{\eta},-d \phi_{\tau}, \delta_{\eta}, H^{\prime}(\eta) \delta_{\eta}, d\left(t H^{\prime}(\eta)\right),-\delta_{t}\right)
$$

By the two previous lemmas, one gets the tangent space $T_{p}\left(M \cap M_{0}\right)+T_{p}\left(M \cap M_{1}\right)$ at the point $p=\left(x, \phi_{x}, \eta, H(\eta),-\phi_{\eta},-\phi_{\tau}, \eta, H(\eta), 0,0\right)$.

Lemma 1.22. At every point $p \in M \cap M_{0} \cap M_{1}$ the tangent space $\left(T_{p} M_{0}+T_{p} M_{1}\right) \cap T_{p} M$ is determined by the following system:

$$
\begin{gathered}
\phi_{\tau}=t, \phi_{\eta}=-t H^{\prime}(\eta), t=0, \\
\phi_{x \eta} \delta_{x}+\phi_{\eta \eta} \delta_{\eta}+\phi_{\tau \eta} \delta_{\tau}=-H^{\prime}(\eta)^{T} \delta_{t}, \\
\phi_{x \tau} \delta_{x}+\phi_{\eta \tau} \delta_{\eta}+\phi_{\tau \tau} \delta_{\tau}=\delta_{t} .
\end{gathered}
$$

Proof. We have

$$
T_{p} M_{0}+T_{p} M_{1}=T_{p}\left(G \times G_{0}\right)+T_{p}\left(G \times G_{1}\right)=T_{p} G \times\left(T_{p} G_{0}+T_{p} G_{1}\right) .
$$


Moreover,

$$
\begin{aligned}
& T_{p} G_{0}=\left(\eta, \tau, 0,0, \delta_{\eta}, \delta_{\tau}, 0,0\right), \\
& T_{p} G_{1}=\left(\eta, H(\eta), t H^{\prime}(\eta),-t ; \delta_{\eta} ; H^{\prime}(\eta) \delta_{\eta}, t H^{\prime \prime}(\eta) \delta_{\eta}+H^{\prime}(\eta)^{T} \delta_{t},-\delta_{t}\right) .
\end{aligned}
$$

This yields $T_{p} G_{0}+T_{p} G_{1}=\left(\eta, H(\eta), 0,0, \delta_{\eta}, \delta_{\tau}, H^{\prime}(\eta)^{T} \delta_{t},-\delta_{t}\right)$. Thus, the space $\left(T_{p} M_{0}+\right.$ $\left.T_{p} M_{1}\right) \cap T_{p} M$ depends on $\delta=\left(\delta_{x}, \delta_{\eta}, \delta_{\tau}, \delta_{t}\right)$ such that $d\left(\phi_{\eta}\right)=d\left(t H^{\prime}(\eta)\right), d\left(\phi_{\tau}\right)=\delta_{t}$. The lemma is proved.

We now rewrite the results above in matrix form. At each point

$$
p=\left(x, \phi_{x}, \eta, H(\eta), 0,0, \eta, H(\eta), 0,0\right)
$$

we set

$$
\begin{gathered}
A_{0}=\left(\begin{array}{cccc}
\phi_{x \eta} & \phi_{\eta \eta} & \phi_{\tau \eta} & H^{\prime}(\eta)^{T} \\
\phi_{x \tau} & \phi_{\eta \tau} & \phi_{\tau \tau} & -I \\
0 & 0 & 0 & I
\end{array}\right), A_{1}=\left(\begin{array}{cccc}
\phi_{x \eta} & \phi_{\eta \eta} & \phi_{\tau \eta} & H^{\prime}(\eta)^{T} \\
\phi_{x \tau} & \phi_{\eta \tau} & \phi_{\tau \tau} & -I \\
0 & H^{\prime}(\eta) & -I & 0
\end{array}\right), \\
A=\left(\begin{array}{llll}
\phi_{x \eta} & \phi_{\eta \eta} & \phi_{\tau \eta} & H^{\prime}(\eta)^{T} \\
\phi_{x \tau} & \phi_{\eta \tau} & \phi_{\tau \tau} & -I
\end{array}\right) .
\end{gathered}
$$

So we have

- the tangent space $T_{p}\left(M_{0} \cap M\right)$ depends on the parameter $\delta=\left(\delta_{x}, \delta_{\eta}, \delta_{\tau}, \delta_{t}\right)$ such that $A_{0} \delta=0$

- the tangent space $T_{p}\left(M_{1} \cap M\right)$ is defined by $A_{1} \delta=0$;

- the space $\left(T_{p} M_{0}+T_{p} M_{1}\right) \cap T_{p} M$ depends on $\delta$ such that $A \delta=0$.

Condition (1L.4) is equivalent to

$$
\operatorname{ker} A_{0}+\operatorname{ker} A_{1}=\operatorname{ker} A \text {. }
$$

Therefore, $M, M_{0}, M_{1}$ is an intersecting triple if and only if

$$
\operatorname{rank}\left(\begin{array}{cccc}
\phi_{x \eta} & \phi_{\eta \eta} & \phi_{\tau \eta} & H^{\prime}(\eta)^{T} \\
\phi_{x \tau} & \phi_{\eta \tau} & \phi_{\tau \tau} & -I \\
0 & H^{\prime}(\eta) & -I & 0 \\
0 & 0 & 0 & I
\end{array}\right)=\operatorname{rank} A_{0}+\operatorname{rank} A_{1}-\operatorname{rank} A
$$


This condition is equivalent to the following

$$
\begin{gathered}
\operatorname{rank}\left(\begin{array}{cccc}
\phi_{x \eta} & \phi_{\eta \eta} & \phi_{\tau \eta} & H^{\prime}(\eta)^{T} \\
\phi_{x \tau} & \phi_{\eta \tau} & \phi_{\tau \tau} & -I \\
0 & H^{\prime}(\eta) & -I & 0
\end{array}\right)-\operatorname{rank}\left(\begin{array}{ccc}
\phi_{x \eta} & \phi_{\eta \eta} & \phi_{\tau \eta} \\
\phi_{x \tau} & \phi_{\eta \tau} & \phi_{\tau \tau} \\
0 & H^{\prime}(\eta) & -I
\end{array}\right)= \\
\operatorname{rank}\left(\begin{array}{llll}
\phi_{x \eta} & \phi_{\eta \eta} & \phi_{\tau \eta} & H^{\prime}(\eta)^{T} \\
\phi_{x \tau} & \phi_{\eta \tau} & \phi_{\tau \tau} & -I
\end{array}\right)-\operatorname{rank}\left(\begin{array}{ccc}
\phi_{x \eta} & \phi_{\eta \eta} & \phi_{\tau \eta} \\
\phi_{x \tau} & \phi_{\eta \tau} & \phi_{\tau \tau}
\end{array}\right) .
\end{gathered}
$$

Remark 1.23. Note that $\phi_{1}(x, \eta)=\phi(x, \eta, H(\eta))$ is the phase function parametrizing $\Lambda_{1}$. Hence,

$$
\begin{aligned}
& \operatorname{rank}\left(\begin{array}{cccc}
\phi_{x \eta} & \phi_{\eta \eta} & \phi_{\tau \eta} & H^{\prime}(\eta)^{T} \\
\phi_{x \tau} & \phi_{\eta \tau} & \phi_{\tau \tau} & -I \\
0 & H^{\prime}(\eta) & -I & 0
\end{array}\right) \\
= & \operatorname{rank}\left(\phi_{x \eta}+\phi_{x \tau} H^{\prime}(\eta) \quad \phi_{\eta \eta}+\phi_{\eta \tau} H^{\prime}(\eta)+H^{\prime}(\eta)^{T} \phi_{\tau \eta}+H^{\prime}(\eta)^{T} \phi_{\tau \tau} H^{\prime}(\eta)\right)+2 l \\
= & \operatorname{rank}\left(\begin{array}{ll}
\phi_{1 x \eta} & \phi_{1 \eta \eta}
\end{array}\right)+2 l .
\end{aligned}
$$

This leads to the definition of multiphase functions:

Definition 1.24. Let $\phi: X \times\left(\mathbb{R}^{k+l} \backslash 0\right) \rightarrow \mathbb{R}$ be homogeneous of degree 1 with respect to $\theta=(\eta, \tau)$.

- The function $\phi$ is called a degenerate multiphase function if conditions (‥14) and ([L.18) hold. Specifically, this means

1. Both $\phi_{0}(x, \theta)=\phi(x, \eta, \tau)$ and $\phi_{1}(x, \eta)=\phi(x, \eta, 0)$ are clean phase functions with excesses $e_{0}, e_{1}$, respectively.

2. On the critical set $C_{\phi}=\left\{(x, \eta, \tau): \phi_{\eta}(x, \eta, \tau)=0, \phi_{\tau}(x, \eta, \tau)=0, \tau=0\right\}$ we have

$$
\operatorname{rank}\left(\begin{array}{cc}
\phi_{x \eta} & \phi_{\eta \eta} \\
\phi_{x \tau} & \phi_{\eta \tau}
\end{array}\right)=k+l-e, \operatorname{rank}\left(\phi_{x \eta} \quad \phi_{\eta \eta} \quad \phi_{\tau \eta}\right)=k-\left(e_{0}+e_{1}-e\right) .
$$

The numbers $\left(e_{0}, e_{1}, e\right)$ will be called the excesses of the multiphase function $\phi$.

- If $e_{0}=e_{1}=e$ then we say $\phi$ is a clean multiphase function with excess $e$.

- The multiphase function $\phi$ is regular if all excesses are zero.

Example 1.25. Assume that $X=\mathbb{R}^{n}$ and $x=\left(x^{\prime}, x^{\prime \prime}\right) \in \mathbb{R}^{n-l} \times \mathbb{R}^{l}$. Then function $\phi(x, \eta, \tau)=x^{\prime} \eta+x^{\prime \prime} \tau$ is a regular multiphase function parametrizing $\Lambda_{0}=\left\{\left(0,0 ; \xi^{\prime}, \xi^{\prime \prime}\right)\right\}$, 
$\Lambda_{1}=\left\{\left(x^{\prime}, 0 ; 0, \xi^{\prime \prime}\right)\right\}$

Set $\tilde{\eta}=\left(\eta, \omega^{\prime}\right), \tilde{\tau}=\left(\tau, \omega^{\prime \prime}\right), \omega=\left(\omega^{\prime}, \omega^{\prime \prime}\right) \in \mathbb{R}^{r^{\prime}} \times \mathbb{R}^{r^{\prime \prime}}$,

$$
\psi(x, \tilde{\eta}, \tilde{\tau})=\phi(x, \eta, \tau)+\frac{1}{2|\eta, \tau|}\langle Q \omega, \omega\rangle,
$$

where $Q=\left(\begin{array}{cc}A & B \\ B^{\prime} & C\end{array}\right)$ is a symmetric matrix in $\mathbb{R}^{r^{\prime}+r^{\prime \prime}}$. Then $\psi$ is a multiphase function parametrizing the same pair of Lagrangian submanifolds as $\phi$ if and only if $\operatorname{rank} A+$ $\operatorname{rank} Q=\operatorname{rank}(A B)+\operatorname{rank}(A B)^{\prime}$. Especially, if $r^{\prime}=r^{\prime \prime}$ and $Q$ is a non-degenerate, then the condition above holds if and only if $A=0$ and $B$ is invertible. For instance, $A=0, C=$ $0, B=I$, then $\psi$ is a multiphase function with excess $(0, r, r)$.

When $r^{\prime}=0$ and $C$ is invertible, $\psi$ is not a multiphase function although $\psi_{0}, \psi_{1}$ and $\psi$ are phase functions parametrizing the Lagrangian pair $\Lambda_{0}, \Lambda_{1}$ and the isotropic submanifold $\Lambda_{0} \cap \Lambda_{1}$.

As an immediate corollary of Theorem $\llbracket .7$ and the definition above, we get the following statement:

Proposition 1.26. Let $\phi$ be a degenerate multiphase function and $\Lambda_{j}$ be the Lagrangian submanifold parametrized by $\phi_{j}$. Then $\Lambda_{0}$ intersects $\Lambda_{1}$ cleanly in an isotropic submanifold of dimension $n-\left(l+e_{0}+e_{1}-2 e\right)$. Especially, a clean multiphase function parametrizes an intersecting pair of Lagrangian submanifolds with $(n-l)$-dimensional intersection.

Remark 1.27. In comparison with clean phase functions for isotropic submanifolds, we recall the definition in [BG81]: The function $\phi(x, \theta)$ is a clean (isotropic) phase function if:

i) $Z=\left\{(x, \theta) \mid \phi_{\theta}(x, \theta)=0, \tau=0\right\}$ is a submanifold of $X \times \mathbb{R}^{N}$ of codimension $m$,

ii) At each point of $Z$ exact $m$ of the differentials $d \phi_{\theta}, d \tau$ are linearly independent.

iii) Each differential $d \tau_{j}$ is linear combination of $d x, d \phi_{x}, d \phi_{\eta}$,

Note that the condition iii) means $\left(\begin{array}{ll}\phi_{x \eta} & \phi_{\eta \eta} \\ \phi_{x \tau} & \phi_{\eta \tau}\end{array}\right)=\operatorname{rank}\left(\phi_{x \eta}, \phi_{\eta \eta}\right)+l$.

Hence, clean multiphase functions can be represented as isotropic clean phase functions satisfying some additional conditions as following:

The function $\phi(x, \eta, \tau)$ is a multiphase function if the following three conditions hold:

1. $\Lambda_{\phi}$ is composable with $G_{0}, G_{1}$, i.e., $\Lambda_{\phi} \times G_{j}$ intersects $T^{*} X \times \Delta_{T^{*} S}$ cleanly. This condition is equivalent to that $\phi_{0}(x, \eta, \tau)=\phi(x, \eta, \tau)$ and $\phi_{1}(x, \eta)=\phi(x, \eta, H(\eta))$ are clean Lagrangian phase functions. 
2. $\Lambda_{\phi}$ is composable with $G_{0} \cap G_{1}$, i.e. $\Lambda_{\phi} \times\left(G_{0} \cap G_{1}\right)$ intersects $T^{*} X \times \Delta_{T^{*} S}$ cleanly and condition $e)$ in Theorem 1.4. This condition implies that $\Lambda_{\phi} \circ\left(G_{0} \cap G_{1}\right)$ is an isotropic submanifold of $T^{*} X \backslash 0$. So $\phi(x, \theta)$ is a clean phase function for some isotropic manifold.

3. The rank condition which ensures that $\Lambda_{\phi} \circ\left(G_{0} \cap G_{1}\right)=\left(\Lambda_{\phi} \circ G_{0}\right) \cap\left(\Lambda_{\phi} \circ G_{1}\right)$.

Roughly speaking, $\phi$ is a clean multiphase function if $\phi_{0}, \phi_{1}$ and $\phi$ are clean phase functions with the same excess and satisfy an additional dimension condition.

Theorem 1.28. Let $C$ be a homogeneous canonical relation from $T^{*} Y \backslash 0$ to $T^{*} X \backslash 0$ and $\left(\Lambda_{0}, \Lambda_{1}\right)$ a pair of Lagrangian submanifolds of $T^{*} Y \backslash 0$. Assume that $\left(C \times \Lambda_{0}, C \times \Lambda_{1}, T^{*} X \times\right.$ $\left.\Delta_{T^{*} Y}\right)$ is an intersecting triple. Then $C \circ \Lambda_{0}$ and $C \circ \Lambda_{1}$ intersect cleanly.

Moreover, if $\Phi(x, y, \xi)$ is a regular phase function defining $C$ and $(\phi(y, \eta, \tau), H(\tau))$ is a regular multiphase function parametrizing $\left(\Lambda_{0}, \Lambda_{1}\right)$, then $(\Phi(x, y, \xi)+\varphi(y, \eta, \tau), H(\tau))$ is a multiphase function parametrizing $\left(C \circ \Lambda_{0}, C \circ \Lambda_{1}\right)$.

Proof. The first statement follows from Theorem 1.3. Now we proof the second. Set

$$
\psi(x, y, \xi, \tau, \eta)=\Phi(x, y, \xi)+\varphi(y, \eta, \tau)
$$

We regard $y$ as the fibre variable and introduce the new variable $\omega=\left(y\left(\xi^{2}+\eta^{2}\right)^{1 / 2}, \xi, \eta\right)$. Under the assumption of intersecting triple, we obtain that $\psi_{0}(x, \omega, \tau)=\psi(x, \omega, \tau)$ and $\psi_{1}(x, \omega)=\psi(x, \omega, H(\omega))$ are phase functions parametrizing $C \circ \Lambda_{0}, C \circ \Lambda_{1}$. So it is sufficient to check the rank condition. Let $\Lambda_{\psi}=\left(x, \psi_{x} ; \omega, \tau,-\psi_{\omega},-\psi_{\tau}\right), \tilde{G}_{0}=(\omega, \tau ; 0,0)$, $\tilde{G}_{1}=\left(\omega, H(\eta), t H_{\omega},-t\right), E=T^{*} X \times \Delta_{T^{*} \mathbb{R}^{N}}$, where $(\omega, \tau) \in \mathbb{R}^{N}, N=n_{y}+n_{\xi}+n_{\eta}+n_{\tau}$.

We need to show that

$$
\left[T_{p}\left(\Lambda_{\psi} \times \tilde{G}_{0}\right)+T_{p}\left(\Lambda_{\psi} \times \tilde{G}_{1}\right)\right] \cap T_{p} E=T_{p}\left(\Lambda_{\psi} \times \tilde{G}_{0} \cap E\right)+T_{p}\left(\Lambda_{\psi} \times \tilde{G}_{1} \cap E\right),
$$

for all $p \in\left(\Lambda_{\psi} \times \tilde{G}_{0}\right) \cap\left(\Lambda_{\psi} \times \tilde{G}_{1}\right) \cap E$.

First, we check that

$$
\left(\Lambda_{\psi} \times \tilde{G}_{j}\right) \cap E \equiv\left(C \times \Lambda_{j}\right) \cap T^{*} X \times \Delta_{T * Y} .
$$

This follows from the standard argument in composition theorem. In fact, $p \in\left(\Lambda_{\psi} \times \tilde{G}_{0}\right) \cap$ 
$E$ if and only if $(x, y, \xi, \eta, \tau)$ satisfies

$$
\begin{aligned}
\Phi_{y}(x, y, \xi)+\varphi_{y}(y, \eta, \tau) & =0, \\
\Phi_{\xi}(x, y, \xi) & =0, \\
\varphi_{\eta}(y, \eta, \tau) & =0, \\
\varphi_{\tau}(y, \eta, \tau) & =0 .
\end{aligned}
$$

This means that $\left\{\left(x, \Phi_{x}, y,-\Phi_{y}\right) \in C \mid\left(y, \varphi_{y}\right) \in \Lambda_{0} ;\left(y,-\Phi_{y}\right)=\left(y, \phi_{y}\right)\right\}$. This is nothing but $C \times \Lambda_{0} \cap\left(T^{*} X \times \Delta_{T * Y}\right)$. (Note that $\Phi$ and $\varphi_{0}$ are regular phase functions.)

Then we show that

$$
\left[T_{p}\left(\Lambda_{\psi} \times \tilde{G}_{0}\right)+T_{p}\left(\Lambda_{\psi} \times \tilde{G}_{1}\right)\right] \cap T_{p} E \simeq\left[T_{p}\left(C \times \Lambda_{0}\right)+T_{p}\left(C \times \Lambda_{1}\right)\right] \cap T_{p}\left(T^{*} X \times \Delta_{T^{*} Y}\right) .
$$

Indeed, let $\Delta_{y}=T_{p}\left(T^{*} X \times \Delta_{T^{*} Y}\right), \Delta_{\eta, \tau}=T_{p}\left(T^{*} Y \times \Delta_{T^{*} \mathbb{R}_{\eta, \tau}}\right)$, we have

$$
\begin{aligned}
& {\left[T_{p}\left(\Lambda_{\psi} \times \tilde{G}_{0}\right)+T_{p}\left(\Lambda_{\psi} \times \tilde{G}_{1}\right)\right] \cap T_{p} E } \\
= & {\left[T_{p} C \times\left[T_{p} \Lambda_{\varphi} \times\left(T_{p} G_{0}+T_{p} G_{1}\right) \cap \Delta_{\eta, \tau}\right]\right] \cap \Delta_{y} } \\
= & {\left[T_{p} C \times\left[T_{p}\left(\Lambda_{\varphi} \times G_{0}\right) \cap \Delta_{\eta, \tau}\right]+\left[T_{p}\left(\Lambda_{\varphi} \times G_{1}\right) \cap \Delta_{\eta, \tau}\right]\right] \cap \Delta_{y} } \\
= & {\left[T_{p} C \times\left[T_{p} \Lambda_{0}+T_{p} \Lambda_{1}\right]\right] \cap \Delta_{y} } \\
= & {\left[T_{p}\left(C \times \Lambda_{0}\right)+T_{p}\left(C \times \Lambda_{1}\right)\right] \cap \Delta_{y} } \\
= & {\left[T_{p}\left(C \times \Lambda_{0}\right) \cap \Delta_{y}\right]+\left[T_{p}\left(C \times \Lambda_{1}\right) \cap \Delta_{y}\right] . }
\end{aligned}
$$

Finally, equations $(1.20),(1.25)$ imply that $\psi, H$ satisfy the rank condition.

\subsubsection{The equivalence of multiphase functions}

In the classical theory, the pull-back of a fibre preserving diffeomorphism maps the classes of phase functions in some conic neighbourhood into another one with the same excess defining on another conic neighbourhood of the same dimension. Unfortunately, this property does not hold in the classes of multiphase functions. This forces us to put additional conditions on the transformations. Because of this, the usual procedure for reducing the fibre dimension and eliminating the excess of phase functions cannot be applied directly to the multiphase functions. In this part, we shall introduce the notion of admissible transformation such that its pull-back acts between classes of multiphase functions with the same excess. We also give other basic transforms on the classes of multiphase 
functions: increasing, decreasing the fibre dimension and eliminating the excesses. Combining with the invariant of the symbol estimate, we obtain the local representation of paired Lagrangian distributions of multiphase functions. We postpone proving this result until the end of this chapter, after investigating the class of symbols and the representation of paired Lagrangian distributions via oscillatory integrals.

Now we study the relation between two multiphase functions parametrizing the same pair of Lagrangian submanifolds. Assume that $\Gamma, \tilde{\Gamma}$ are open conic sets in $X \times\left(\mathbb{R}^{N-l} \times \mathbb{R}^{l} \backslash 0\right)$ and $X \times\left(\mathbb{R}^{\tilde{N}-l} \times \mathbb{R}^{l} \backslash 0\right)$. Let $\phi, \tilde{\phi}$ be clean multiphase functions in $\Gamma, \tilde{\Gamma}$, respectively.

Definition 1.29. A map $\Gamma \ni(x, \eta, \tau) \mapsto(x, \tilde{\eta}(x, \eta, \tau), \tilde{\tau}(x, \eta, \tau)) \in \tilde{\Gamma}$ is an admissible transformation if the following conditions hold:

1. The functions $\tilde{\eta}(x, \eta, \tau), \tilde{\tau}(x, \eta, \tau)$ are smooth and homogeneous of degree 1 with respect to $(\eta, \tau)$.

2. The matrices $D_{\eta} \tilde{\eta}(x, \eta, 0),(x, \eta, 0) \in \Gamma$ and $\mathcal{D}_{\tau} \tilde{\tau}(x, \eta, \tau),(x, \eta, \tau) \in \Gamma$ are invertible.

3. The function $\tilde{\tau}(x, \eta, \tau)$ vanishes on the set $\tau=0$.

The map $\chi$ is admissible near $\left(x_{0}, \eta_{0}, 0\right)$ if $\chi$ is an admissible transformation in some conic neighbourhood $V \subseteq \Gamma$ of $\left(x_{0}, \eta_{0}, 0\right)$.

Remark 1.30. A fibre preserving diffeomorphism $(x, \eta, \tau) \mapsto(x, g(x, \eta, \tau), h(x, \eta, \tau))$ satisfying $h(x, \eta, 0)=0$ is admissible near $\left(x_{0}, \eta_{0}, 0\right) \in \Gamma$.

We restrict the class of transformations because in general, the pull-back of multiphase function under a fibre preserving transformation is no longer a multiphase function. However, this fact holds if we put an extra condition on transformation as the following result.

Lemma 1.31. Assume that $\phi(x, \eta, \tau)$ is a multiphase function near $\left(x_{0}, \eta_{0}, 0\right)$ with excesses $\left(e_{0}, e_{1}, e\right)$ and $\chi$ is an admissible transformation such that $\chi\left(x_{0}, \tilde{\eta}_{0}, 0\right)=\left(x_{0}, \eta_{0}, 0\right)$. Let define $\psi(x, \eta, \tau)=\phi(\chi(x, \eta, \tau))$ in some conic neighbourhood of $\left(x_{0}, \tilde{\eta}_{0}, 0\right)$. Then $\psi$ is a multiphase function with the excesses $\left(e_{0}, e_{1}, e\right)$ parametrizing the same Lagrangian pair as $\phi$.

Proof. Since $\psi_{0}(x, \eta, \tau)=\phi_{0}(\chi(x, \eta, \tau))$ and $\psi_{1}(x, \eta)=\phi_{1}(\chi(x, \eta, 0))$ and $\chi$ and $\left.\chi\right|_{\tau=0}$ are differmorphisms, we first conclude that $\psi_{0}, \psi_{1}$ are phase functions with the same excesses 
and parametrizes the same Lagrangian manifolds as $\phi_{0}, \phi_{1}$. Hence, we only need to check

$$
\operatorname{rank}\left(\begin{array}{ll}
\psi_{x \eta} & \psi_{\eta \eta} \\
\psi_{x \tau} & \psi_{\eta \tau}
\end{array}\right)=\operatorname{rank}\left(\begin{array}{cc}
\phi_{x \eta} & \phi_{\eta \eta} \\
\phi_{x \tau} & \phi_{\eta \tau}
\end{array}\right), \operatorname{rank}\left(d \psi_{\eta}\right)=\operatorname{rank}\left(d \phi_{\eta}\right) .
$$

At the point $(x, \eta, 0) \in \Gamma$ satisfying $\phi_{\eta}=0, \phi_{\tau}=0$ we have

$$
\left(\begin{array}{ll}
\psi_{x \eta} & \psi_{\eta \eta} \\
\psi_{x \tau} & \psi_{\eta \tau}
\end{array}\right)=\left(\begin{array}{ll}
\tilde{\eta}_{\eta} & \tilde{\tau}_{\eta} \\
\tilde{\eta}_{\tau} & \tilde{\tau}_{\tau}
\end{array}\right)\left(\begin{array}{ccc}
\phi_{x \eta} & \phi_{\eta \eta} & \phi_{\eta \tau} \\
\phi_{x \tau} & \phi_{\eta \tau} & \phi_{\tau \tau}
\end{array}\right)\left(\begin{array}{cc}
I & 0 \\
\tilde{\eta}_{x} & \tilde{\eta}_{\eta} \\
\tilde{\tau}_{x} & \tilde{\tau}_{\eta}
\end{array}\right) .
$$

Because $\tilde{\tau}_{x}$ and $\tilde{\tau}_{\eta}$ vanish at the point $(x, \eta, 0)$, we obtain:

$$
\left(\begin{array}{cc}
\psi_{x \eta} & \psi_{\eta \eta} \\
\psi_{x \tau} & \psi_{\eta \tau}
\end{array}\right)=\left(\begin{array}{cc}
\tilde{\eta}_{\eta} & 0 \\
\tilde{\eta}_{\tau} & \tilde{\tau}_{\tau}
\end{array}\right)\left(\begin{array}{cc}
\phi_{x \eta} & \phi_{\eta \eta} \\
\phi_{x \tau} & \phi_{\eta \tau}
\end{array}\right)\left(\begin{array}{cc}
I & 0 \\
\tilde{\eta}_{x} & \tilde{\eta}_{\eta}
\end{array}\right)
$$

This implies that rank $\left(\begin{array}{ll}\psi_{x \eta} & \psi_{\eta \eta} \\ \psi_{x \tau} & \psi_{\eta \tau}\end{array}\right)=\operatorname{rank}\left(\begin{array}{ll}\phi_{x \eta} & \phi_{\eta \eta} \\ \phi_{x \tau} & \phi_{\eta \tau}\end{array}\right)$. Similarly, the equality

$$
\left(\begin{array}{lll}
\psi_{x \eta} & \psi_{\eta \eta} & \psi_{\eta \tau}
\end{array}\right)=\left(\begin{array}{lll}
\tilde{\eta}_{\eta}
\end{array}\right)\left(\begin{array}{ccc}
\phi_{x \eta} & \phi_{\eta \eta} & \phi_{\eta \tau}
\end{array}\right)\left(\begin{array}{ccc}
I & 0 & 0 \\
\tilde{\eta}_{x} & \tilde{\eta}_{\eta} & \tilde{\eta}_{\tau} \\
\tilde{\tau}_{x} & \tilde{\tau}_{\eta} & \tilde{\tau}_{\tau}
\end{array}\right)
$$

yields the remain condition. This proves the lemma.

We shall use admissible transformation to define the equivalence between multiphase functions.

Definition 1.32. Let $\phi$ and $\tilde{\phi}$ be multiphase functions in some conic neighbourhoods $\Gamma, \tilde{\Gamma}$. Two multiphase functions $\phi, \tilde{\phi}$, are said to be equivalent if there exists an admissible transformation $\chi$ from $\Gamma$ to $\Gamma^{\prime}$ such that $\phi=\chi^{*} \tilde{\phi}$.

These functions are said to be locally equivalent near $\left(x_{0}, \eta_{0}, 0\right)$ and $\left(x_{0}, \tilde{\eta}_{0}, 0\right)$ if $\chi$ is locally admissible near these points and $\chi\left(x_{0}, \eta_{0}, 0\right)=\left(x_{0}, \tilde{\eta}_{0}, 0\right)$.

Remark 1.33. Since $g(x, \eta, 0)=0, D_{\eta} f(x, \eta, 0)$ and $D_{\tau} g(x, \eta, \tau)$ are invertible in some conic neighbourhood $\tau /|\eta| \leq \varepsilon$. So the necessary condition for equivalence of multiphase functions is that $\phi_{0}, \tilde{\phi}_{0}$ and $\phi, \tilde{\phi}$ are equivalent phase functions in the Hörmander's sense.

Proposition 1.34. Let $\phi(x, \eta, \tau)$ and $\tilde{\phi}(x, \tilde{\eta}, \tilde{\tau})$ be regular multiphase functions in conic neighbourhoods of $\left(x_{0}, \eta_{0}, 0\right) \in X \times\left(\mathbb{R}^{N} \backslash 0\right)$ and $\left(x_{0}, \tilde{\eta}_{0}, 0\right) \in X \times\left(\mathbb{R}^{\tilde{N}} \backslash 0\right)$, respectively. Then $\phi$ and $\tilde{\phi}$ are equivalent in some neighbourhoods of these points if the following conditions hold: 
1. $\phi, \tilde{\phi}$ parametrize the same pair of Lagrangian submanifolds,

2. $N=\tilde{N}, l=\tilde{l}$,

3. $\phi_{\eta \eta}\left(x_{0}, \eta_{0}, 0\right)$ and $\tilde{\phi}_{\tilde{\eta} \tilde{\eta}}\left(x, \tilde{\eta}_{0}, 0\right)$ have the same signature.

Proof. Our proof is based on the proof of the equivalence of phase functions in [Ho85]. First step. We can assume that $\eta=\tilde{\eta}, \phi(x, \eta, 0)=\tilde{\phi}(x, \eta, 0)$. Indeed, since $\phi_{1}, \tilde{\phi}_{1}$ parametrize the same $\Lambda_{1}, N-l=\tilde{N}-\tilde{l}$ and $\phi_{1 \eta \eta}, \tilde{\phi}_{1 \eta \eta}$ have the same signature, we conclude that $\phi_{1}$ and $\tilde{\phi}_{1}$ are equivalent phase functions. Hence, there is a diffeomorphism $(x, \eta) \mapsto(x, f(x, \eta))$ such that $\phi_{1}(x, \eta)=\tilde{\phi}_{1}(x, f(x, \eta))$. So the multiphase function $\varphi(x, \eta, \tau)=\tilde{\phi}(x, f(x, \eta), \tau)$ is equivalent to $\tilde{\phi}$ and $\varphi(x, \eta, 0)=\phi(x, \eta, 0)$. So we may assume that $\phi, \tilde{\phi}$ have the mentioned property.

Second step. We prove that by changing $\tau$-variable, we have $\phi_{\tau}(x, \eta, 0)=\phi_{\tilde{\tau}}(x, \eta, 0)$.

On the set $\left\{\phi_{\eta}(x, \eta, 0)=0\right\}$ the system $\left\{\phi_{\tau}(x, \eta, 0)=0\right\}$ is equivalent to $\left\{\tilde{\phi}_{\tau}(x, \eta, 0)=0\right\}$, which leads to

$$
\phi_{\tau}(x, \eta, 0)=\tilde{\phi}_{\tau}(x, \eta, 0) a(x, \eta)+\tilde{\phi}_{\eta}(x, \eta, 0) b(x, \eta),
$$

for $a=\left(a_{i j}(x, \eta)\right)_{l, l}$ and $b=\left(b_{i j}(x, \eta)_{N-l, l}\right)$ are smoothly homogeneous of degree zero. Differentiating both sides of $\mathbb{1 . 2 6}$ with respect to $x, \eta$ at the point $\left(x_{0}, \eta_{0}, 0\right)$ and adding $\phi_{\eta x}, \phi_{\eta \eta}$, we obtain

$$
\left(\begin{array}{ll}
\phi_{\tau x} & \phi_{\eta x} \\
\phi_{\tau \eta} & \phi_{\eta \eta}
\end{array}\right)=\left(\begin{array}{ll}
\tilde{\phi}_{\tau x} & \tilde{\phi}_{\eta x} \\
\tilde{\phi}_{\tau \eta} & \tilde{\phi}_{\eta \eta}
\end{array}\right)\left(\begin{array}{cc}
a & 0 \\
b & I
\end{array}\right) .
$$

Since $\phi$ and $\tilde{\phi}$ are regular multiphase functions, both matrices have rank $N$. Therefore $a$ is invertible at $\left(x_{0}, \eta_{0}\right)$. One has a non-degenerate extension in some conic neighbourhood of $\left(x_{0}, \eta_{0}, 0\right)$. Consider a diffeomorphism $\chi:(x, \eta, \tau) \mapsto(x, \eta+b \tau, a \tau)$ in some neighbourhood of $|\tau| /|\eta| \leq \varepsilon$, then the multiphase function $\chi^{*} \tilde{\phi}$ is equivalent to $\tilde{\phi}$ and differs from $\phi$ a term which vanishes to second order in $\tau$.

Third step. Without loss of generality, we assume that $\phi-\tilde{\phi}$ vanishes to second order on $C_{\phi}=\left\{(x, \eta, \tau) \mid \phi_{\eta}=0, \phi_{\tau}=0\right\}$ and on $\{\tau=0\}$. In fact, consider the map

$$
(x, \eta, \tau) \rightarrow\left(x, \phi_{x}, \phi_{\eta}\right)
$$


Because $\phi$ satisfies the rank condition, it is injective. By the implicit theorem, there is a smooth map $\Psi$ from a neighbourhood of $\left(x_{0}, \xi_{0}, 0\right)$ to $\mathbb{R}^{N}$ such that

$$
(\eta, \tau)=\Psi\left(x, \phi_{x}, \phi_{\tau}\right)
$$

Replace $\Psi$ by $\Psi\left(x, \xi\left|\xi_{0}\right| /|\xi|, \omega\right)|\xi| /\left|\xi_{0}\right|$, we may assume $\Psi$ is homogeneous of degree 1 in $\xi$. Consider the transformation

$$
\chi:(x, \eta, \tau) \rightarrow\left(x, \tilde{\Psi}\left(x, \phi_{x}, \phi_{\eta}\right)\right),
$$

where $\tilde{\Psi}$ is the map corresponding to $\tilde{\phi}$. It is fibre preserving and its restriction to $C_{\phi}$ is a differmorphism. Two functions $\chi^{*} \tilde{\phi}, \phi$ have the desired assumption. It remains to check that $\chi^{*} \tilde{\phi}$ and $\tilde{\phi}$ are locally equivalent. Set $\xi=\phi_{x}, \omega=\phi_{\eta}$ then the differential of $\xi$ at a fixed $x$ has the form

$$
\tilde{\Psi}_{\xi}\left(\phi_{x \eta}, \phi_{x \tau}\right)+\tilde{\Psi}_{\omega}\left(\phi_{\eta \eta}, \phi_{\eta \tau}\right)=\tilde{\Psi}_{\xi}\left(\tilde{\phi}_{x \eta}, \tilde{\phi}_{x \tau}\right)+\tilde{\Psi}_{\omega}\left(\tilde{\phi}_{\eta \eta}, \tilde{\phi}_{\eta \tau}\right)=I,
$$

since at the point $(x, \eta, 0)$, we have $\phi(x, \eta, 0)=\tilde{\phi}(x, \eta, 0), \phi_{\tau}(x, \eta, 0)=\tilde{\phi}_{\tau}(x, \eta, 0)$ and $(\eta, \tau)=\tilde{\Psi}\left(x, \tilde{\phi}_{x}, \tilde{\phi}_{\eta}\right)$. This implies that $\chi$ is a diffeomorphism at $\left(x_{0}, \eta_{0}, 0\right)$ and moreover, $\chi(x, \eta, 0)=(x, \eta, 0)$. Thus $\tilde{\phi}$ and $\chi^{*} \tilde{\phi}$ are equivalent multiphase functions. So we may assume that $\tilde{\phi}, \phi$ vanish to second order on $C_{\phi}$ and $\tau=0$.

Forth step. Now we are in position to use Hörmander's method for phase functions $\phi(x, \eta, \tau)$ and $\tilde{\phi}(x, \eta, \tau)$. Using Taylor's expansion, we obtain

$$
\phi(x, \theta)-\tilde{\phi}(x, \theta)=\phi_{\theta} A(x, \theta) \phi_{\theta},
$$

where $\theta=(\eta, \tau)$ and $A$ is a symmetric matrix vanishing at $\tau=0$. We shall show that there exists a diffeomorphism transformation of the form

$$
(x, \theta) \rightarrow\left(x, \theta+B(x, \theta) \phi_{\theta}\right)
$$

which satisfies $\phi(x, \theta)=\tilde{\phi}\left(x, \theta+B \phi_{\theta}\right)$ and $B(x, \eta, 0)=0$. In fact, since $\phi\left(x, \theta+B \phi_{\theta}\right)-$ $\phi(x, \theta)=\phi(x, \theta)+\phi_{\theta} B \phi_{\theta}+\phi_{\theta} B G B \phi_{\theta}$, where $G$ is a matrix depending smoothly on $x, \theta$ and $B$. We take $B$ as the solution of the equation

$$
B+B G B=A .
$$

This equation has a unique solution if $A$ is small enough and moreover, this solution is homogeneous of degree 1 in $\theta$. Since $A(x, \eta, 0)=0$, we conclude that whenever $|\tau| /|\eta| \leq \varepsilon$ small enough, we can take $B$ as the solution of the equation above and $B(x, \eta, 0)=0$. So $\phi$ and $\tilde{\phi}$ are equivalent in some conic neighbourhood of $\left(x_{0}, \eta_{0}, 0\right)$. This completes the proof. 
Proposition 1.35. Let $\phi(x, \eta, \tau)$ be a multiphase function in a conic neighbourhood of $\left(x_{0}, \eta_{0}, 0\right)$ with excesses $e_{0}=0, e_{1}=e>0$. Then there exists a multiphase function $\psi$ near $\left(x_{0}, \eta_{0}^{\prime}, \tau_{0}^{\prime}\right)$ which parametrizes the same Lagrangian pair as $\phi$ such that

$$
\phi(x, \eta, \tau)=\psi\left(x, \eta^{\prime}, \tau^{\prime}\right)+\eta^{\prime \prime} \tau^{\prime \prime} /\left|\eta^{\prime}, \tau^{\prime}\right|,\left(\eta^{\prime \prime}, \tau^{\prime \prime}\right) \in \mathbb{R}^{e_{1}} \times \mathbb{R}^{e_{1}}
$$

Proof. First of all, since $\phi_{1}(x, \eta)=\phi(x, \eta, 0)$ is a clean phase function with excess $e_{1}$ near $\left(x_{0}, \eta_{0}\right)$, we may assume that there exists a variable splitting $\eta=\left(\eta^{\prime}, \eta^{\prime \prime}\right) \in \mathbb{R}^{k-e_{1}} \times \mathbb{R}^{e_{1}}$ such that $\phi_{\eta^{\prime \prime}}(x, \eta, 0)=0$. Now using the condition $\operatorname{rank}\left(d \phi_{\eta}\left(x_{0}, \eta_{0}, 0\right)\right)=k$, we derive that $\left(\phi_{\eta^{\prime \prime} \tau}\right)$ has full rank. So there exists $\tau=\left(\tau^{\prime}, \tau^{\prime \prime}\right)$ such that $\phi_{\eta^{\prime \prime} \tau^{\prime \prime}}\left(x_{0}, \eta_{0}, 0\right)$ has rank $e_{1}$. It follows that the matrix

$$
\left(\begin{array}{ll}
\phi_{\eta^{\prime \prime} \eta^{\prime \prime}} & \phi_{\tau^{\prime \prime} \eta^{\prime \prime}} \\
\phi_{\tau^{\prime \prime} \eta^{\prime \prime}} & \phi_{\tau^{\prime \prime} \tau^{\prime \prime}}
\end{array}\right)
$$

has the full rank $e_{1}$. Hence, the equations $\phi_{\eta^{\prime \prime}}(x, \eta, \tau)=0, \phi_{\tau^{\prime \prime}}(x, \eta, \tau)=0$ are solved near $\left(x_{0}, \eta_{0}, 0\right)$ by $\eta^{\prime \prime}=g\left(x, \eta^{\prime}, \tau^{\prime}\right), \tau^{\prime \prime}=h\left(x, \eta^{\prime}, \tau^{\prime}\right)$. Because $\phi_{\eta^{\prime \prime}}(x, \eta, 0)=0$, we obtain $h\left(x, \eta^{\prime}, 0\right)=0$. Now if we use the change of variables

$$
(x, \eta, \tau) \mapsto\left(x, \eta^{\prime}, \eta^{\prime \prime}-g\left(x, \eta^{\prime}, \tau^{\prime}\right), \tau^{\prime}, \tau^{\prime \prime}-h(x, \eta, \tau)\right),
$$

then the new function $\tilde{\phi}(x, \eta, \tau)=\phi\left(x, \eta^{\prime}, \tau^{\prime \prime}+g\left(x, \eta^{\prime}, \tau^{\prime}\right), \tau^{\prime}, \tau^{\prime \prime}+h\left(x, \eta^{\prime}, \tau^{\prime}\right)\right)$ is an equivalent multiphase function of $\phi$ satisfying $d_{\eta^{\prime \prime}, \tau^{\prime \prime}} \tilde{\phi}\left(x, \eta^{\prime}, 0, \tau^{\prime}, 0\right)=0$. Without loss of generality, we suppose that $g=0, h=0$. Define $\psi\left(x, \eta^{\prime}, \tau^{\prime}\right)=\phi\left(x, \eta^{\prime}, 0, \tau^{\prime}, 0\right)$. It is clear that the matrix $\left(\begin{array}{ll}\psi_{x \eta^{\prime}} & \psi_{\eta^{\prime}, \eta^{\prime}} \\ \psi_{x \tau^{\prime}} & \phi_{\eta^{\prime} \tau^{\prime}}\end{array}\right)$ at $\left(x_{0}, \eta_{0}^{\prime}, 0\right)$ has full rank $k-e_{1}+l-e_{1}$. Combining with the fact that $\psi\left(x, \eta^{\prime}, 0\right)$ and $\psi\left(x, \eta^{\prime}, \tau^{\prime}\right)$ are regular phase functions parametrizing the same Lagrangian submanifolds as $\phi_{0}, \phi_{1}$, we conclude that $\psi$ is a regular multiphase function which parametrizes the same Lagrangian pair as $\phi$. Now we shall prove that under a suitable transform on $\eta^{\prime \prime}, \tau^{\prime \prime}$, two multiphase functions $\phi$ and $\psi$ have the desired relation. Using Taylor's expansion, we have

$$
\phi(x, \eta, \tau)=\phi\left(x, \eta^{\prime}, 0, \tau^{\prime}, 0\right)+\frac{1}{2\left|\eta^{\prime}, \tau^{\prime}\right|}\left[\left(A \eta^{\prime \prime}, \eta^{\prime \prime}\right)+\left(B \eta^{\prime \prime}, \tau^{\prime \prime}\right)+B\left(\tau^{\prime \prime}, \eta^{\prime \prime}\right)+\left(C \tau^{\prime \prime}, \tau^{\prime \prime}\right)\right],
$$

where $A, C$ are symmetric matrices and $B\left(x_{0}, \eta_{0}^{\prime}, \tau_{0}^{\prime}, 0\right)=\left|\eta_{0}^{\prime}, \tau_{0}^{\prime}\right| \phi_{\eta^{\prime \prime} \tau^{\prime \prime}}\left(x, \eta_{0}, \tau_{0}\right)$ is invertible. Set $G=\left(\begin{array}{ll}A & B \\ B^{\prime} & C\end{array}\right), Q=\left(\begin{array}{ll}0 & I \\ I & 0\end{array}\right)$. We look for a matrix $R$ of the form

$$
R=\left(\begin{array}{cc}
B^{\prime}-C Z / 2 & C / 2 \\
Z & I
\end{array}\right)
$$


which satisfies $R^{\prime} Q R=G$. Or equivalently, the matrix $Z$ solves the equation

$$
B Z+Z^{\prime} B^{\prime}-Z^{\prime} C Z=A
$$

We claim that this matrix equation has a unique solution provided that $B$ is invertible and $B^{-1} A$ is small. In fact, consider $A$ as a functional of $Z$. The differential when $Z=0$ is the mapping

$$
\frac{d A}{d Z}(0)(V)=B V+V^{\prime} B^{\prime}-V^{\prime} C Z-\left.Z^{\prime} C V\right|_{Z=0} .
$$

It is surjective as for all symmetric $U$, the matrix $V=B^{-1} U / 2$ solves the equation $V^{\prime} B^{\prime}+$ $B V=U$. By the implicit function theorem, we have $Z=Z(A)$ near $A=0$. Now at the point $\left(x_{0}, \eta_{0}, 0\right)$ we have $A=0$ and $B$ is invertible. There exists a conic neighbourhood of $\left(x_{0}, \eta_{0}, 0\right)$ such that $B$ is still invertible and $A$ is small enough. So applying the change of variables

$$
\left(\begin{array}{l}
\tilde{\eta}^{\prime \prime} \\
\tilde{\tau}^{\prime \prime}
\end{array}\right)=\left(\begin{array}{cc}
B^{\prime}-C Z / 2 & C / 2 \\
Z & I
\end{array}\right)\left(\begin{array}{l}
\eta^{\prime \prime} \\
\tau^{\prime \prime}
\end{array}\right),
$$

we obtain $\phi\left(x, \eta^{\prime}, \tilde{\eta}^{\prime \prime}, \tau^{\prime}, \tilde{\tau}^{\prime \prime}\right)=\psi\left(x, \eta^{\prime}, \tau^{\prime}\right)+\frac{\tilde{\eta}^{\prime \prime} \cdot \tilde{\tau}^{\prime \prime}}{\left|\eta^{\prime}, \tau^{\prime}\right|}$. Finally, we need to check that the transformation $(x, \eta, \tau) \mapsto\left(x, \eta^{\prime}, \tilde{\eta}^{\prime \prime}, \tau^{\prime}, \tilde{\tau}^{\prime \prime}\right)$ is admissible. It is sufficient to show that $Z(x, \eta, \tau)=0$ when $\tau=0$. This condition holds because $A(x, \eta, 0)=0$. So $\phi(x, \eta, \tau)=$ $\psi\left(x, \eta^{\prime}, \tau^{\prime}\right)+\eta^{\prime \prime} \cdot \tau^{\prime \prime} /\left|\eta^{\prime}, \tau^{\prime}\right|$.

\subsubsection{Reduction of multiphase functions}

In this part, we shall prove some results in the change of the fibre dimension. We use the same idea as in the case of phase functions. In dealing with multiphase functions, we need further properties of the change of coordinates. Therefore, in the proof of reduction for multiphase functions, whenever we use a change of coordinates, we have to verify the admissibility of the transformation. This demands a careful treatment, sometimes the transformation does not come from an explicit formula. We shall prove the main result in several steps.

Proposition 1.36. Let $\phi$ be a multiphase function with excesses $\left(e_{0}, e_{1}, e\right)$ in some conic neighbourhood in $X \times\left(\mathbb{R}^{k+l} \backslash 0\right)$ of $\left(x_{0}, \eta_{0}, 0\right)$. Then there exists an admissible transformation

$$
(x, \eta, \tau) \mapsto\left(x, \eta^{\prime}, \eta^{\prime \prime}, \tau^{\prime}, \tau^{\prime \prime}\right),
$$


and a multiphase function with excesses $\left(e_{0}, e_{0}+e_{1}-e, e_{0}\right)$ parametrizing the same Lagrangian pair as $\phi$ such that

$$
\phi(x, \eta, \tau)=\psi\left(x, \eta^{\prime}, \tau^{\prime}\right)+\eta^{\prime \prime} \tau^{\prime \prime} /\left|\eta^{\prime}, \tau^{\prime}\right|
$$

where $\eta=\left(\eta^{\prime}, \eta^{\prime \prime}\right) \in \mathbb{R}^{k-\left(e-e_{0}\right)} \times \mathbb{R}^{e-e_{0}}, \tau=\left(\tau^{\prime}, \tau^{\prime \prime}\right) \in \mathbb{R}^{l-\left(e-e_{0}\right)} \times \mathbb{R}^{e-e_{0}}$.

Proof. First of all, since $\phi_{1}(x, \eta)=\phi(x, \eta, 0)$ is a clean phase function with excess $e_{1}$ near $\left(x_{0}, \eta_{0}\right)$, we may assume that there exists a variable splitting $\eta=\left(\eta^{\prime}, \eta^{\prime \prime}\right) \in \mathbb{R}^{k-e_{1}} \times \mathbb{R}^{e_{1}}$ such that $\phi_{\eta^{\prime \prime}}(x, \eta, 0)=0$. By the conditions $\operatorname{rank}\left(d \phi_{\eta}\left(x_{0}, \eta_{0}, 0\right)\right)=k-\left(e_{0}+e_{1}-e\right)$ we conclude that $\left(\phi_{\eta^{\prime \prime} \tau}\right)$ has rank at least $e-e_{0}$. So there exists $\tau=\left(\tau^{\prime}, \tau^{\prime \prime}\right) \in \mathbb{R}^{l-\left(e-e_{0}\right)} \times \mathbb{R}^{e-e_{0}}$ such that $\phi_{\eta^{\prime \prime} \tau^{\prime \prime}}\left(x_{0}, \eta_{0}, 0\right)$ invertible, and then

$$
\left(\begin{array}{ll}
\phi_{\eta^{\prime \prime} \eta^{\prime \prime}} & \phi_{\tau^{\prime \prime} \eta^{\prime \prime}} \\
\phi_{\tau^{\prime \prime} \eta^{\prime \prime}} & \phi_{\tau^{\prime \prime} \tau^{\prime \prime}}
\end{array}\right)
$$

has the full rank $2\left(e-e_{0}\right)$. Hence, the equations $\phi_{\eta^{\prime \prime}}(x, \eta, \tau)=0, \phi_{\tau^{\prime \prime}}(x, \eta, \tau)=0$ are solved near $\left(x_{0}, \eta_{0}, 0\right)$ by $\eta^{\prime \prime}=g\left(x, \eta^{\prime}, \tau^{\prime}\right), \tau^{\prime \prime}=h\left(x, \eta^{\prime}, \tau^{\prime}\right)$. Since $\phi_{\eta^{\prime \prime}}(x, \eta, 0)=0$, we obtain $h\left(x, \eta^{\prime}, 0\right)=0$. We now use the change of variables

$$
(x, \eta, \tau) \mapsto\left(x, \eta^{\prime}, \eta^{\prime \prime}-g\left(x, \eta^{\prime}, \tau^{\prime}\right), \tau^{\prime}, \tau^{\prime \prime}-h(x, \eta, \tau)\right) .
$$

The new function $\tilde{\phi}(x, \eta, \tau)=\phi\left(x, \eta^{\prime}, \tau^{\prime \prime}+g\left(x, \eta^{\prime}, \tau^{\prime}\right), \tau^{\prime}, \tau^{\prime \prime}+h\left(x, \eta^{\prime}, \tau^{\prime}\right)\right)$ is an equivalent multiphase function of $\phi$ satisfying $d_{\eta^{\prime \prime}, \tau^{\prime \prime}} \tilde{\phi}\left(x, \eta^{\prime}, 0, \tau^{\prime}, 0\right)=0$. Without loss of generality, we suppose that $g=0, h=0$. Let define $\psi\left(x, \eta^{\prime}, \tau^{\prime}\right)=\phi\left(x, \eta^{\prime}, 0, \tau^{\prime}, 0\right)$. At $\left(x_{0}, \eta_{0}, 0\right)$ we have

$$
\left(\begin{array}{ccccc}
\phi_{x \eta^{\prime}} & \phi_{\eta^{\prime} \eta^{\prime}} & \phi_{\eta^{\prime \prime} \eta^{\prime}} & \phi_{\tau^{\prime \prime} \eta^{\prime}} & \phi_{\tau^{\prime} \eta^{\prime}} \\
\phi_{x \eta^{\prime \prime}} & \phi_{\eta^{\prime} \eta^{\prime \prime}} & \phi_{\eta^{\prime \prime} \eta^{\prime \prime}} & \phi_{\tau^{\prime \prime} \eta^{\prime \prime}} & \phi_{\tau^{\prime} \eta^{\prime \prime}} \\
\phi_{x \tau^{\prime \prime}} & \phi_{\eta^{\prime} \tau^{\prime \prime}} & \phi_{\eta^{\prime \prime} \tau^{\prime \prime}} & \phi_{\tau^{\prime \prime} \tau^{\prime \prime}} & \phi_{\tau^{\prime} \tau^{\prime \prime}} \\
\phi_{x \tau^{\prime}} & \phi_{\eta^{\prime} \tau^{\prime}} & \phi_{\eta^{\prime \prime} \tau^{\prime}} & \phi_{\tau^{\prime \prime} \tau^{\prime}} & \phi_{\tau^{\prime} \tau^{\prime}}
\end{array}\right)=\left(\begin{array}{ccccc}
\phi_{x \eta^{\prime}} & \phi_{\eta^{\prime} \eta^{\prime}} & 0 & 0 & \phi_{\tau^{\prime} \eta^{\prime}} \\
0 & 0 & 0 & \phi_{\tau^{\prime \prime} \eta^{\prime \prime}} & 0 \\
0 & 0 & \phi_{\eta^{\prime \prime} \tau^{\prime \prime}} & \phi_{\tau^{\prime \prime} \tau^{\prime \prime}} & 0 \\
\phi_{x \tau^{\prime}} & \phi_{\eta^{\prime} \tau^{\prime}} & 0 & 0 & \phi_{\tau^{\prime} \tau^{\prime}}
\end{array}\right) .
$$

Hence, $\psi$ is a degenerate multiphase function with the excesses $\left(e_{0}, e_{0}+e_{1}-e, e_{0}\right)$ which parametrizes the same Lagrangian pair as $\phi$. Now we shall prove that under a suitable transform on $\eta^{\prime \prime}, \tau^{\prime \prime}$, two multiphase functions $\phi$ and $\psi$ have the desired relation. Using Taylor's expansion, we have

$$
\phi(x, \eta, \tau)=\phi\left(x, \eta^{\prime}, 0, \tau^{\prime}, 0\right)+\frac{1}{2\left|\eta^{\prime}, \tau^{\prime}\right|}\left[\left(A \eta^{\prime \prime}, \eta^{\prime \prime}\right)+\left(B \eta^{\prime \prime}, \tau^{\prime \prime}\right)+B\left(\tau^{\prime \prime}, \eta^{\prime \prime}\right)+\left(C \tau^{\prime \prime}, \tau^{\prime \prime}\right)\right],
$$


where $A, C$ are symmetric matrices, $B\left(x_{0}, \eta_{0}^{\prime}, \tau_{0}^{\prime}, 0\right)=\left|\eta_{0}^{\prime}, \tau_{0}^{\prime}\right| \phi_{\eta^{\prime \prime} \tau^{\prime \prime}}\left(x, \eta_{0}, \tau_{0}\right)$ is invertible. Let $G=\left(\begin{array}{ll}A & B \\ B^{\prime} & C\end{array}\right), Q=\left(\begin{array}{ll}0 & I \\ I & 0\end{array}\right)$ and $R=\left(\begin{array}{cc}B^{\prime}-C Z / 2 & C / 2 \\ Z & I\end{array}\right)$.

Using the same argument as in the proof of Proposition [1.35, we obtain the existence of matrix $R$ satisfying $R^{\prime} Q R=G$ and the admissibility of the transformation $(x, \eta, \tau) \mapsto$ $\left(x, \eta^{\prime}, \tilde{\eta}^{\prime \prime}, \tau^{\prime}, \tilde{\tau}^{\prime \prime}\right)$.

Proposition 1.37. Assume that $\phi$ is a multiphase function with excesses $\left(e, e_{1}, e\right)$ in an open conic neighbourhood in $X \times\left(\mathbb{R}^{k+l} \backslash 0\right)$ of $\left(x_{0}, \eta_{0}, 0\right)$. Then after using an admissible transformation, we may split $\eta=\left(\eta^{\prime}, \eta^{\prime \prime}\right) \in \mathbb{R}^{k-e_{1}} \times \mathbb{R}^{e_{1}}$ and $\tau=\left(\tau^{\prime}, \tau^{\prime \prime}\right) \in \mathbb{R}^{l-\left(e-e_{1}\right)} \times \mathbb{R}^{e-e_{1}}$ such that

$$
\phi_{\eta^{\prime \prime}}(x, \eta, \tau)=0, \phi_{\tau^{\prime \prime}}(x, \eta, \tau)=0
$$

Furthermore, for each fixed $\eta^{\prime \prime}, \tau^{\prime \prime}$, the function $\psi\left(x, \eta^{\prime}, \tau^{\prime}\right)=\phi\left(x, \eta^{\prime},\left|\eta^{\prime}, \tau^{\prime}\right| \eta^{\prime \prime}, \tau^{\prime},\left|\eta^{\prime}, \tau^{\prime}\right| \tau^{\prime \prime}\right)$ is a regular multiphase function parametrizing the same Lagrangian pair as $\phi$.

Proof. Since $\phi_{1}(x, \eta)=\phi(x, \eta, 0)$ is a clean phase with excess $e_{1}$, applying the procedure for eliminating excess of phase function, there is a splitting $\eta=\left(\eta^{\prime}, \eta^{\prime \prime}\right) \in \mathbb{R}^{k-e_{1}} \times \mathbb{R}^{e_{1}}$ such that $\phi_{\eta^{\prime \prime}}(x, \eta, 0)=0$. Because $\operatorname{rank}\left(\phi_{x \eta}, \phi_{\eta \eta}, \phi_{\tau \eta}\right)=k-e_{1}$ by multiphase assumption, we conclude that $\phi_{\tau \eta^{\prime \prime}}(x, \eta, 0)=0$. Hence,

$$
\operatorname{rank}\left(\begin{array}{cc}
\phi_{x \eta} & \phi_{\eta \eta} \\
\phi_{x \tau} & \phi_{\eta \tau}
\end{array}\right)=\operatorname{rank}\left(\begin{array}{ccc}
\phi_{x \eta^{\prime}} & \phi_{\eta^{\prime} \eta^{\prime}} & 0 \\
0 & 0 & 0 \\
\phi_{x \tau} & \phi_{\eta^{\prime} \tau} & 0
\end{array}\right)=k+l-e
$$

So we can choose $\tau=\left(\tau^{\prime}, \tau^{\prime \prime}\right) \in \mathbb{R}^{l-\left(e-e_{1}\right)} \times \mathbb{R}^{e-e_{1}}$ such that

$$
\operatorname{rank}\left(\begin{array}{cc}
\phi_{x \eta^{\prime}} & \phi_{\eta^{\prime} \eta^{\prime}} \\
\phi_{x \tau^{\prime}} & \phi_{\eta^{\prime} \tau^{\prime}}
\end{array}\right)=k+l-e .
$$

It has some consequences. First, $C_{\phi}$ is determined by $\phi_{\eta^{\prime}}(x, \eta, 0)=0, \phi_{\tau^{\prime}}(x, \eta, 0)=0$. So there exist smooth matrices $f(x, \eta), g(x, \eta)$ such that $\phi_{\tau^{\prime \prime}}(x, \eta, 0)=\phi_{\eta^{\prime \prime}}(x, \eta) f(x, \eta)+$ $\phi_{\tau^{\prime}}(x, \eta) g(x, \eta)$. Extend $f, g$ into some conic neighbourhood of $\left(x_{0}, \eta_{0}, 0\right)$ and consider an admissible transformation of the form

$$
(x, \eta, \tau) \rightarrow\left(x, \eta^{\prime}+f(x, \eta, \tau) \tau^{\prime \prime}, \eta^{\prime \prime}, \tau+g(x, \eta, \tau) \tau^{\prime \prime}, \tau^{\prime \prime}\right) .
$$

In the new coordinates, we have $\phi_{\tau^{\prime \prime}}(x, \eta, 0)=0$. Thus we may assume that $\phi$ satisfies this property. 
Second, using the condition $\operatorname{rank}\left(d \phi_{\eta}, d \phi_{\tau}\right)=k+l-e$, we conclude that $C_{\phi_{0}}$ is determined by $(k+l-e)$ linearly independent functions $\phi_{\eta^{\prime}}(x, \eta, \tau), \phi_{\tau^{\prime}}(x, \eta, \tau)$, and $C_{\phi_{1}}$ is determined by $k+l-e_{1}$ linearly independent functions $\phi_{\eta^{\prime}}(x, \eta, \tau)=0, \tau=0$. Note that $C_{\phi_{0}}$ intersects $C_{\phi_{1}}$ cleanly.

Rewrite $\left(\eta^{\prime \prime}, \tau^{\prime \prime}\right)=\omega=\left(\omega_{1}, \ldots, \omega_{e}\right),(\eta, \tau)=\left(\eta^{\prime}, \tau^{\prime}, \omega\right)$. Since $\phi_{\omega}(x, \eta, 0)=0, \phi_{\omega}$ vanishes on $C_{\phi_{1}}$. Now we shall prove the conclusion of this theorem by induction in the number $e$ of $\omega$-variables. If $e=0$, then $e_{1}=0$. Thus, the theorem is obvious. Suppose that the statement of the theorem holds for multiphase with the excess smaller than $e$. Because the function $\phi_{\omega_{e}}(x, \eta, \tau)$ vanishes on $C_{\phi_{0}}$ and $C_{\phi_{1}}$, there exist smooth functions $p, q$ such that

$$
\phi_{\omega_{e}}(x, \eta, \tau)=\phi_{\eta^{\prime}}(x, \eta, \tau) p(x, \eta, \tau)+\phi_{\tau^{\prime}}(x, \eta, \tau) q(x, \eta, \tau), \quad q(x, \eta, 0)=0 .
$$

Consider the change of coordinates in the form

$$
\chi:(x, \eta, \tau) \mapsto\left(x, \eta^{\prime}+\tilde{p}(x, \eta, \theta), \eta^{\prime \prime}, \tau^{\prime}+\tilde{q}(x, \eta, \tau), \tau^{\prime \prime}\right)
$$

such that $\tilde{p}, \tilde{q}$ are the solutions of the ODE system

$$
\begin{array}{r}
\partial_{\omega_{e}} \tilde{p}(x, \eta, \tau)=p(x, \eta, \tau), \partial_{\omega_{e}} \tilde{q}(x, \eta, \tau)=q(x, \eta, \tau) \\
\left.\tilde{p}(x, \eta, \tau)\right|_{\omega_{e}=0}=0,\left.\tilde{q}(x, \eta, \tau)\right|_{\omega_{e}=0}=0 .
\end{array}
$$

Note that $\tilde{q}(x, \eta, 0)=0$ as $q(x, \eta, 0)=0$. Hence, the transformation $\chi$ in ([1.30) is admissible. In the new variables, $\phi\left(x, \eta^{\prime}, \tau^{\prime}, \omega\right)$ satisfies $\partial_{\omega_{e}} \phi=0$, so the multiphase function $\phi$ does not depend on $\omega_{e}$. Rewrite $\omega^{\prime}=\left(\omega_{0}, \ldots, \omega_{e-1}\right)$ then $\phi(x, \eta, \tau)=\phi\left(x, \eta^{\prime}, \tau^{\prime}, \omega^{\prime}\right)$ is a multiphase function with the excess $e-1$. By induction assumption, using the transform on $\left(x, \eta^{\prime}, \tau^{\prime}, \omega^{\prime}\right)$, we have $\phi_{\omega^{\prime}}(x, \eta, \tau)=0$. Hence, $\partial_{\omega} \phi(x, \eta, \tau)=0$. This completes the proof.

\subsection{Amplitude functions of double orders}

\subsubsection{Definition and basic properties of double symbols}

Definition 1.38. We denote by $S^{m, p}\left(X \times \mathbb{R}^{k} \times \mathbb{R}^{l}\right.$ ) (or $S^{m, p}$ when $X, k, l$ are fixed) the set of all smooth functions $a(x, \eta, \tau)$ such that for an arbitrary compact subset $K \Subset X$ and any multi-indices $\alpha, \beta, \gamma$, there exists a constant $C_{\alpha, \beta, \gamma, K}$ satisfying

$$
\left|\partial_{x}^{\alpha} \partial_{\eta}^{\beta} \partial_{\tau}^{\gamma} a(x, \eta, \tau)\right| \leq C_{\alpha, \beta, \gamma, K}\langle\eta, \tau\rangle^{p-|\beta|}\langle\tau\rangle^{m-p-|\gamma|} .
$$


$S^{m, p}$ is called the space of double symbols of orders $(m, p)$. We set

$$
\begin{array}{ll}
S^{-\infty, p}=\bigcup_{m \in \mathbb{R}} S^{m, p}\left(X \times \mathbb{R}^{k} \times \mathbb{R}^{l}\right), & S^{m,-\infty}=\bigcap_{p \in \mathbb{R}} S^{m, p}\left(X \times \mathbb{R}^{k} \times \mathbb{R}^{l}\right), \\
S^{\infty}=\bigcup_{m, p \in \mathbb{R}} S^{m, p}\left(X \times \mathbb{R}^{k} \times \mathbb{R}^{l}\right), & S^{-\infty}=\bigcap_{m, p \in \mathbb{R}} S^{m, p}\left(X \times \mathbb{R}^{k} \times \mathbb{R}^{l}\right) .
\end{array}
$$

The space of symbols of product type of orders $(m, p)$, denoted by $\tilde{S}^{m, p}\left(X \times \mathbb{R}^{k+l}\right)$, is the set of all smooth functions $a(x, \eta, \tau)$ such that for each compact subset $K \Subset X$ and all multi-indices $\alpha, \beta, \gamma$, there exists a constant $C_{\alpha, \beta, \gamma, K}$ satisfying

$$
\left|\partial_{x}^{\alpha} \partial_{\eta}^{\beta} \partial_{\tau}^{\gamma} a(x, \eta, \tau)\right| \leq C_{\alpha, \beta, \gamma, K}\langle\eta\rangle^{m-|\beta|}\langle\tau\rangle^{p-|\gamma|},(x, \eta, \tau) \in K \times \mathbb{R}^{k+l}
$$

Obviously, $S^{m}\left(X \times \mathbb{R}^{k}\right) \otimes S^{p}\left(X \times \mathbb{R}^{l}\right)$ is a subset of $\tilde{S}^{m, p}\left(X \times \mathbb{R}^{k+l}\right)$.

Proposition 1.39. Let $a(x, \eta, \tau)$ be a smooth function. Define $\tilde{a}(x, \tilde{\eta}, \tau)=a(x, \tilde{\eta}\langle\tau\rangle, \tau)$. Then $a \in S^{m, p}$ if and only if $\tilde{a} \in \tilde{S}^{p, m}$.

Proof. This proposition is proved by a simple computation.

Consequently, we have the following properties:

$$
\begin{gathered}
S_{1,0}^{m} \subset S^{m, m} \text { for all } m \in \mathbb{R}, \\
S^{m, p} \cdot S^{m^{\prime}, p^{\prime}} \subset S^{m+m^{\prime}, p+p^{\prime}} \text { for all } m, p, m^{\prime}, p^{\prime} \in \mathbb{R}, \\
S^{-\infty} \subset S^{m, p} \subset S^{m^{\prime}, p^{\prime}} \subset S^{\infty} \text { if } m \leq m^{\prime}, p \leq p^{\prime}, \\
\partial_{x}^{\alpha} \partial_{\eta}^{\beta} \partial_{\tau}^{\gamma}: S^{m, p} \rightarrow S^{m-|\beta|-|\gamma|, p-|\beta|} \text { for all } \alpha, \beta, \gamma .
\end{gathered}
$$

\subsubsection{Topology of double symbol spaces}

Let $\Gamma$ be a conic subset of $X \times\left(\mathbb{R}^{k} \times \mathbb{R}^{l}\right)$. With any compact cone $K \Subset \Gamma$ and $j \in \mathbb{N}$, we define a semi-norm on $S^{m, p}(\Gamma)$ by

$$
p_{K, m, p, j}(a)=\sup _{\substack{(x, \eta, \tau) \in K,|\alpha|+|\beta|+|\gamma| \leq j}}\left|\partial_{x}^{\alpha} \partial_{\eta}^{\beta} \partial_{\tau}^{\gamma} a(x, \eta, \tau)\right|\langle\eta, \tau\rangle^{-p+|\beta|}\langle\tau\rangle^{-m+p+|\gamma|} .
$$

Proposition 1.40. The space $S^{m, p}$ is a Fréchet space with the natural semi-norms. Let $a \in S^{m, p}$ and $\chi(\eta, \tau)$ be a smooth function which is identical to 1 in $\{|\eta, \tau| \leq 1\}$, vanishes outside $\{|\eta, \tau| \geq 2\}$. Set $a_{\varepsilon}(x, \eta, \tau)=\chi(\varepsilon \eta, \varepsilon \tau) a(x, \eta, \tau), k \geq 1$. Then $a_{\varepsilon} \in S^{-\infty}$ and the sequence $\left\{a_{\varepsilon}\right\}$ converges to a as $\varepsilon \rightarrow 0+$ with respect to the topology of $S^{m^{\prime}, p^{\prime}}$ for all $m \leq m^{\prime}$ and $p-p^{\prime}<0$. 
Lemma 1.41. Let $a \in S^{0,0}\left(X \times \mathbb{R}^{k} \times \mathbb{R}^{l}\right)$ and $0 \leq \epsilon \leq 1$.

a) Set $a_{\epsilon}(x, \eta, \tau)=a(x, \epsilon \eta, \epsilon \tau)$. Then $a_{\epsilon}$ is bounded in $S^{0,0}$, and $a_{\epsilon} \rightarrow a_{0}$ in $S^{m, p}$ for all $m, p>0$ when $\epsilon \rightarrow 0$.

b) Define $b_{\epsilon}(x, \eta, \tau)=a(x, \eta, \epsilon \tau)$. Then $b_{\epsilon}$ tends to $a(x, \eta, 0)$ in $S^{m, 0}$ for every $m>0$ when $\epsilon \rightarrow 0$.

c) Similarly, $a(x, \epsilon \eta, \tau)$ converges to $a(x, 0, \tau)$ in $S^{0, p}$ when $\epsilon \rightarrow 0$ for every $p>0$.

Proof. We first prove part a) with an additional assumption that $m, p \in(0,1]$; the general case follows immediately. Since $a_{0}=a(x, 0,0) \in S^{0,0}$, the conclusion follows by proving that for each compact subset $K$ of $X$ and multi-indices $\alpha, \beta, \gamma$, there exists a constant $C$ satisfying

$$
\langle\eta, \tau\rangle^{|\alpha|-p}\langle\tau\rangle^{|\beta|-(m-p)}\left|\partial_{\eta}^{\alpha} \partial_{\tau}^{\beta} \partial_{x}^{\gamma}(a(x, \epsilon \eta, \epsilon \tau)-a(x, 0,0))\right| \leq C_{\alpha, \beta, \gamma} \epsilon^{\min (m, p)},
$$

for all $(x, \eta, \tau) \in K \times \mathbb{R}^{k+l}$.

Case 1. When $\alpha=0,|\beta| \geq 1$, the left hand side of ([L.37) is estimated by

$$
C\langle\eta, \tau\rangle^{-p}\langle\tau\rangle^{|\beta|-m+p}\langle\epsilon \tau\rangle^{-|\beta|} \epsilon^{|\beta|} \leq C \epsilon^{m}\left(\frac{\epsilon\langle\tau\rangle}{\langle\epsilon \tau\rangle}\right)^{|\beta|-m} \leq C \epsilon^{m} .
$$

Case 2. When $|\alpha| \geq 1$, the left hand side of ([1.37) can be estimated by

$$
\begin{aligned}
& C\langle\eta, \tau\rangle^{|\alpha|-p}\langle\tau\rangle^{|\beta|-m+p}\langle\epsilon \eta, \epsilon \tau\rangle^{-|\alpha|}\langle\epsilon \tau\rangle^{-|\beta|} \epsilon^{|\alpha|+|\beta|} \\
& \leq \begin{cases}C \epsilon^{p}\left(\frac{\epsilon\langle\eta, \tau\rangle}{\langle\epsilon \eta, \epsilon \tau\rangle}\right)^{|\alpha|-p}\left(\frac{\epsilon\langle\tau\rangle}{\langle\epsilon \tau\rangle}\right)^{|\beta|} \leq C \epsilon^{p} & \text { when } p \leq m, \\
C \epsilon^{m}\left(\frac{\epsilon\langle\eta, \tau\rangle}{\langle\epsilon \eta, \epsilon \tau\rangle}\right)^{|\alpha|-m}\left(\frac{\epsilon\langle\tau\rangle}{\langle\epsilon \tau\rangle}\right)^{|\beta|}\left(\frac{\langle\eta, \tau\rangle}{\langle\tau\rangle}\right)^{m-p} \leq C \epsilon^{m} & \text { when } p>m .\end{cases}
\end{aligned}
$$

Case 3. When $\alpha=0, \beta=0$, using Taylor's formula, we can estimate the left hand side of (II.37) by

$$
C\langle\eta, \tau\rangle^{-p}\langle\tau\rangle^{p-m}\left(|\epsilon \eta|\langle\epsilon \eta, \epsilon \tau\rangle^{-1}+|\epsilon \tau|\langle\epsilon \tau\rangle^{-1}\right) \leq C \frac{\langle\eta, \tau\rangle^{-p}\langle\tau\rangle^{p-m}|\epsilon \eta|}{\langle\epsilon \eta, \epsilon \tau\rangle}+C \frac{\langle\tau\rangle^{-m}|\epsilon \tau|}{\langle\epsilon \tau\rangle} .
$$

The second term of the right hand side can be bounded by

$$
C \epsilon\langle\tau\rangle^{1-m} \epsilon^{1-m}\langle\epsilon \tau\rangle^{m-1} \leq C \epsilon^{m} .
$$

The first term can be bounded as the case $|\alpha| \geq 1$ :

$$
C\langle\eta, \tau\rangle^{1-p}\langle\tau\rangle^{p-m}\langle\epsilon \eta, \epsilon \tau\rangle^{-1} \epsilon \leq C \epsilon^{\min (m, p)} .
$$

Hence, the inequality ([1.37) holds for $\alpha=0, \beta=0$. This completes the proof of part a).

The parts b) and c) are similarly proved. 
As a consequence of this lemma, we have

Corollary 1.42. Let $\chi \in C_{0}^{\infty}(\mathbb{R})$ and be equal to 1 near zero. Then $\chi(\epsilon \eta, \epsilon \tau) \rightarrow 1$ in $S^{m, p}$ for every $m>0, p>0$. Similarly, $\chi(\epsilon \tau) \rightarrow 1$ in $S^{m, 0}$ for every $m>0$ and $\chi(\epsilon|\eta| /\langle\tau\rangle) \rightarrow 1$ in $S^{0, p}$ for every $p>0$.

\subsubsection{Admissible transformations}

Consider a fibre preserving mapping

$$
\chi: X \times \mathbb{R}^{k} \times \mathbb{R}^{l} \rightarrow X \times \mathbb{R}^{k} \times \mathbb{R}^{l},(x, \eta, \tau) \mapsto(x, f(x, \eta, \tau), g(x, \eta, \tau)) .
$$

Now we shall provide a condition on $\chi$ such that under its pulling back, the double symbol spaces are stable.

Lemma 1.43. Let $a(x, \eta, \tau) \in S^{m, p}, f \in S^{1,1}$ and $g \in S^{1,0}$. Assume that

$$
\langle f, g\rangle \asymp\langle\eta, \tau\rangle ; \quad\langle g\rangle \asymp\langle\tau\rangle .
$$

Then the function $b$ defined by $b(x, \eta, \tau)=a(x, f(x, \eta, \tau), g(x, \eta, \tau))$ belongs to $S^{m, p}$.

Proof. By induction on $|\alpha|+|\beta|+|\gamma|$ one obtains that $\partial_{x}^{\alpha} \partial_{\eta}^{\beta} \partial_{\tau}^{\gamma} b$ is a finite sum of terms of the form

$$
\partial_{x}^{\alpha^{\prime}} \partial_{f}^{\beta^{\prime}} \partial_{g}^{\gamma^{\prime}} a(x, f, g) \prod_{r=1}^{R} \partial_{x}^{\alpha^{\prime \prime}} \partial_{\eta}^{\beta^{\prime \prime}} \partial_{\tau}^{\gamma^{\prime \prime}} f(x, \eta, \tau) \prod_{s=1}^{S} \partial_{x}^{\alpha^{\prime \prime \prime}} \partial_{\eta}^{\beta^{\prime \prime \prime}} \partial_{\tau}^{\gamma^{\prime \prime \prime}} g(x, \eta, \tau),
$$

where $R=\left|\beta^{\prime}\right|, S=\left|\gamma^{\prime}\right|$ and

$$
|\beta| \leq \sum_{r}\left|\beta_{r}^{\prime \prime}\right|+\sum_{s}\left|\beta_{s}^{\prime \prime \prime}\right|, \quad|\gamma| \leq \sum_{r}\left|\gamma_{r}^{\prime \prime}\right|+\sum_{s}\left|\gamma_{s}^{\prime \prime \prime}\right|
$$

The modulus of ([1.39) is bounded by a constant times

$$
\begin{array}{r}
\langle f, g\rangle^{p-\left|\beta^{\prime}\right|}\langle g\rangle^{m-p-\left|\gamma^{\prime}\right|}\langle\eta, \tau\rangle^{R-\sum_{r}\left|\beta_{r}^{\prime \prime}\right|}\langle\tau\rangle^{-\sum_{r}\left|\gamma_{r}^{\prime \prime}\right|}\langle\eta, \tau\rangle^{-\sum_{r}\left|\beta_{r}^{\prime \prime \prime}\right|}\langle\tau\rangle^{S-\sum_{r}\left|\gamma_{r}^{\prime \prime \prime}\right|} \\
\leq\langle\eta, \tau\rangle^{p-|\beta|}\langle\tau\rangle^{m-p-|\gamma|} .
\end{array}
$$

and the result follows.

Remark 1.44. In application, we are in the following situation: $f, g$ is homogeneous of degree 1 in $\eta, \tau$, and

$$
|f, g| \asymp|\eta, \tau| ; \quad|g| \asymp|\tau| .
$$


Let $\chi(t)$ be a cut-off function near zero, i.e., $\chi \in C^{\infty}(\mathbb{R}), \chi(t)=0$ if $t \leq 1 / 2, \chi(t)=1$ for $|t| \geq 1$. Then $b(x, \eta, \tau)=\chi(|\eta, \tau|) a(x, f(x, \eta, \tau), g(x, \eta, \tau)) \in S^{m, p}$. The proof is similar, using the fact that in the support of $\chi, f \in S_{1,0}^{1} \subset S^{1,1}$. Since $g(x, \eta, 0)=0$, there is a matrix of homogeneous function $A(x, \eta, \tau)$ such that $g(x, \eta, \tau)=A(x, \eta, \tau) \tau$. This yields $g \in S^{1,0}$.

Now we prove the invariance of the classes of these symbols under an admissible transformation.

Corollary 1.45. Let $\chi: X \times \mathbb{R}^{k} \times \mathbb{R}^{l} \rightarrow X \times \mathbb{R}^{k} \times \mathbb{R}^{l},(x, \eta, \tau) \mapsto(x, f(x, \eta, \tau), g(x, \eta, \tau))$ be a homogeneous diffeomorphic transformation such that $g(x, \eta, 0)=0$. If $a \in S^{m, p}$ and $|\tau| \leq \epsilon|\eta|$ for $(x, \eta, \tau) \in \operatorname{supp} a$, then $b(x, \eta, \tau)=a(x, f(x, \eta, \tau), g(x, \eta, \tau)) \in S^{m, p}$.

Proof. First, since $D(f, g) / D(\eta, \tau)$ is invertible for fixed $x$, using Euler's relation, we have $(f, g)=D(f, g) / D(\eta, \tau) \cdot(\eta, \tau)$. This implies that $|f, g| \asymp|\eta, \tau|$.

For $\tau=0$, we have $g(x, \eta, 0)=0$. Hence, $D_{\eta} g(x, \eta, 0)=0$. Therefore, $D_{\eta} f(x, \eta, 0)$ is invertible since $D_{\eta} f(x, \eta, 0)$ has full rank. Similarly, we also obtain the invertibility of $D_{\tau} g(x, \eta, 0)$. By continuity, this holds for $|\tau| /|\eta| \leq \varepsilon$. So we have

$$
g_{j}(x, \eta, \tau)=\tau D_{\tau} g_{j}(x, \eta, s \tau), s \in(0,1) .
$$

Because $D_{\tau} g_{j}(x, \eta, \tau)$ is homogeneous of degree zero, we may assume that it is invertible for all $|\tau| /|\eta| \leq \varepsilon$. So we conclude $|g|=\left|\tau D_{\tau} g\right| \geq c|\tau|$. On the other hand, since $g(x, \eta, 0)=$ 0 , there is a matrix $a(x, \eta, \tau)$ whose elements are homogeneous of degree zero such that

$$
g(x, \eta, \tau)=a(x, \eta, \tau) \tau
$$

This implies that $|g| \leq c|\tau|$. So we obtain $|g| \simeq|\tau|$. Applying the remark above, we obtain the conclusion of the corollary.

\subsubsection{Asymptotic expansions of double symbols}

Proposition 1.46. Let $a_{j} \in S^{m_{j}, p_{j}}$ be a sequence of double symbols. Set $m_{j}^{\prime}=\operatorname{supp}_{h \geq j} m_{h}, p_{j}=$ $\operatorname{supp}_{h \geq j} p_{h}$.

a) If $\max _{j} m_{j}=m \in \mathbb{R}$ and $p_{j} \searrow-\infty$, then one can find $a \in S^{m, p_{0}}$ such that for every $J$

$$
\left(a-\sum_{0 \leq j<J-1} a_{j}\right) \in S^{m, p_{J}}
$$


The function a is uniquely determined modulo $S^{m,-\infty}$ and we write $a \sim \sum_{j} a_{j}$.

b) If $\max _{j} p_{j}=p \in \mathbb{R}$ and $m_{j} \searrow-\infty$ as $j \rightarrow \infty$, then there exists a function $a \in S^{m, p}$, $m=\max _{j} m_{j}$ uniquely modulo $S^{-\infty, p}$ such that

$$
\left(a-\sum_{0 \leq j<J}\right) a_{j} \in S^{m_{J}, p} .
$$

We write $a=\sum_{j} a_{j}$ modulo $S^{-\infty, p}$.

c) If $m_{j}, p_{j} \searrow-\infty$ as $j \rightarrow \infty$, then there exists a function $a \in S^{m, p}$ uniquely modulo $S^{-\infty}$ such that

$$
\left(a-\sum_{0 \leq j<J} a_{j}\right) \in S^{m_{J}, p_{J}}
$$

We write $a=\sum_{j} a_{j}$ modulo $S^{-\infty}$.

Proof. We first prove the statement $c$ ). Take a smooth function $\chi \in C_{0}^{\infty}\left(\mathbb{R}^{k+l}\right)$ which is equal to 1 near zero. We can choose a decreasing positive sequence $\epsilon_{j}$ converging to 0 so rapidly in the sense that

$$
\left|\partial_{\alpha}^{\theta} \partial_{x}^{\beta}(1-\chi(x, \epsilon \theta)) a_{j}(x, \theta)\right| \leq 2^{-j}\langle\eta, \tau\rangle^{p_{j}+p-\left|\alpha^{\prime}\right|}\langle\tau\rangle^{m_{j}-p_{j}+m-p-\left|\alpha^{\prime \prime}\right|}, \theta=(\eta, \tau),|\alpha|+|\beta| \leq j .
$$

Let $a_{j}^{\prime}(x, \theta)=(1-\chi(x, \epsilon \theta)) a_{j}(x, \theta)$ and define $a=\sum_{j} a_{j}^{\prime}$. Then $a \in C^{\infty}$ because this sum is locally finite. Given $\alpha, \beta, J$, we can choose $N$ satisfying $N \geq|\alpha|+|\beta|$ and $m_{N} \leq$ $m_{J}+m, p_{N} \leq p_{J}+p$. Then we have

$$
\left|\partial_{\theta}^{\alpha} \partial_{x}^{\beta}\left(a-\sum_{0 \leq j<J} a_{j}^{\prime}\right)\right| \leq\langle\eta, \tau\rangle^{p_{J}-\left|\alpha^{\prime}\right|}\langle\tau\rangle^{m_{J}-p_{J}-\left|\alpha^{\prime \prime}\right|} .
$$

Since $\left(a_{j}-a_{j}^{\prime}\right) \in S^{-\infty}$ and $a_{j}^{\prime} \in S^{m_{j}, p_{j}}$ when $j \geq J$, we conclude that

$$
\left(a-\sum_{0 \leq j<J-1} a_{j}\right) \in S^{m_{J}, p_{J}} .
$$

The uniqueness of $a$ is obvious from this property.

The statements $b$ ) and $c$ ) are proved similarly.

Using the method of stationary phase, one easily obtains the following result:

Proposition 1.47. Suppose that $a(x, \eta, \tau) \in S^{m, p}\left(\mathbb{R}^{k} \times \mathbb{R}^{l}\right)$ and $\eta=\left(\eta^{\prime}, \eta^{\prime \prime}\right) \in \mathbb{R}^{k-r} \times \mathbb{R}^{r}$, $\tau=\left(\tau^{\prime}, \tau^{\prime \prime}\right) \in \mathbb{R}^{l-r} \times \mathbb{R}^{r}$. Assume that exists $\varepsilon>0$ such that

$$
(x, \eta, \tau) \in \operatorname{supp} a \Longrightarrow\left|\eta^{\prime \prime}\right| /\left|\eta^{\prime}, \tau^{\prime}\right| \leq \varepsilon .
$$

$\operatorname{Set} b\left(x, \eta^{\prime}, \tau^{\prime}\right)=\int_{\mathbb{R}^{2 r}} e^{i \eta^{\prime \prime} \tau^{\prime \prime} /\left|\eta^{\prime}, \tau^{\prime}\right|} a(x, \eta, \tau) d \eta^{\prime \prime} \tau^{\prime \prime}$. Then $b \in S^{m+r, p+r}\left(X \times \mathbb{R}^{k-r} \times \mathbb{R}^{l-r}\right)$.

Moreover, one has the following asymptotic expansion

$$
\left.b\left(x, \eta^{\prime}, \tau^{\prime}\right) \sim \sum_{|\alpha|} \frac{i^{\alpha}}{\alpha !} \partial_{\eta^{\prime \prime}}^{\alpha} \partial_{\tau^{\prime \prime}}^{\alpha} a(x, \eta, \tau)\right|_{\eta^{\prime \prime}=0, \tau^{\prime \prime}=0} \operatorname{modulo} S^{m+r,-\infty} .
$$




\subsection{Local representation of paired Lagrangian distribu- tions}

In this section we prove that using multiphase functions and symbols in class $S^{\infty}=$ $\cup_{m, p \in \mathbb{R}} S^{m, p}$, one can define a class of paired Lagrangian distributions via oscillatory integrals.

\subsubsection{Local definition and micro-local properties}

Let $X$ be an open subset of $\mathbb{R}^{n}, \phi(x, \eta, \tau) \in C^{\infty}\left(X \times\left(\mathbb{R}^{N} \backslash 0\right)\right)$ be a multiphase function, and $a \in S^{m, p}\left(X \times \mathbb{R}^{N-l} \times \mathbb{R}^{l}\right)$. Consider the integral

$$
I(\phi, a) u:=\iint e^{i \phi(x, \eta, \tau)} a(x, \eta, \tau) u(x) d \eta d \tau d x, u \in C_{0}^{\infty}(X) .
$$

It is clear that this integral converges absolutely when $m<-N, p<-(N-l)$.

Lemma 1.48. Let $\phi(x, \eta, \tau)$ be a multiphase function. There exists a first-order differential operator

$$
L=\sum_{j=1}^{N-l} p(x, \eta, \tau) \partial_{\eta_{j}}+\sum_{j=1}^{l} q(x, \eta, \tau) \partial_{\tau_{j}}+\sum_{j=1}^{n} r_{j}(x, \eta, \tau) \partial_{x_{j}}+s(x, \eta, \tau),
$$

with coefficients $p_{j} \in S^{0}, q_{j} \in S^{0,-1}, r_{j}, s \in S^{-1,-1}$ such that $L\left(e^{i \phi}\right)=e^{i \phi}$. Moreover, the formal adjoint operator ${ }^{t} L: S^{m, p} \rightarrow S^{m-1, p-1}$ for all $m, p \in \mathbb{R}$.

Proof. Take an arbitrary cut-off function $\chi(\theta) \in C_{0}^{\infty}\left(\mathbb{R}^{N}\right)$ such that $\chi$ is identical to 1 when $|\theta| \leq 1$ and vanishes outside $|\theta| \geq 2$. Then define

$$
L:=\frac{1-\chi(\eta, \tau)}{i D}\left(|\eta, \tau|^{2} \sum_{j=1}^{N-l} \phi_{\eta_{j}} \partial_{j}+|\tau|^{2} \sum_{j=1}^{l} \phi_{\tau_{j}} \partial_{\tau_{j}}+\sum_{j=1}^{n} \phi_{x_{j}} \partial_{x_{j}}\right)+\chi(\eta, \tau),
$$

where $D(x, \eta, \tau):=\left|\phi_{x}\right|^{2}+|\eta, \tau|^{2}\left|\phi_{\eta}\right|^{2}+|\tau|^{2}\left|\phi_{\tau}\right|^{2}$. Note that $D$ is homogeneous in $(\eta, \tau)$ and $D \neq 0$ for all $(\eta, \tau) \neq 0$, so the definition of $L$ is well-defined. Indeed, because $D$ is homogeneous of degree 2 in $\eta, \tau$, we may assume that $|\eta, \tau|=1$. If $\phi_{\eta}=0, \phi_{\tau}=0$, then $\phi_{x} \neq 0$ since $\phi(x, \eta, \tau)$ is a phase function. If $|\tau|=0$, then $\left|\phi_{x}(x, \eta, 0)\right|+\left|\phi_{\eta}(x, \eta, 0)\right| \neq 0$ since $\phi(x, \eta, 0)$ is a phase function. This implies that $D \neq 0$. Now the remain case is $\phi_{\tau} \neq 0$ and $\tau \neq 0$, then it is obvious that $D \geq|\tau|^{2}\left|\phi_{\tau}\right|^{2}>0$. Therefore, $D \neq 0$ as we need. 
Now it is easy to check that

$$
\begin{aligned}
L\left(e^{i \phi}\right) & =e^{i \phi}, \\
p_{j}(x, \eta, \tau) & =i \frac{(1-\chi)|\eta, \tau|^{2} \phi_{\eta_{j}}(x, \eta, \tau)}{D} \in S^{0} \subset S^{0,0}, \\
q_{j}(x, \eta, \tau) & =i|\eta|^{2} \cdot \frac{(1-\chi) \phi_{\tau_{j}}}{D} \in S^{2,0} \cdot S^{-2,-2} \subset S^{0,-2} \subset S^{0,-1}, \\
r_{j}(x, \eta, \tau) & =i \frac{(1-\chi(\eta, \tau)) \phi_{\tau_{j}}(x, \eta, \tau)}{D} \in S^{-1} \subset S^{-1,-1}, \\
s(x, \eta, \tau) & =\chi(\eta, \tau) \in S^{-1,-1} .
\end{aligned}
$$

For each $a(x, \eta, \tau) \in S^{m, p}$, we have

$$
{ }^{t} L a=\sum_{j}^{N-l} \partial_{\eta_{j}}\left(p_{j} a\right)+\sum_{j}^{l} \partial_{\tau_{j}}\left(q_{j} a\right)+\sum_{j}^{n} \partial_{x_{j}}\left(r_{j} a\right)+s a
$$

Combining with properties of symbols, we conclude that ${ }^{t} L$ acts continuously from $S^{m, p}$ to $S^{m-1, p-1}$.

Using the operator $L$, just as in the classical theory of oscillatory integral, we have the following result:

Theorem 1.49. The definition of integral (L.4U) can be extended in a unique way to all $a \in S^{m, p}$ and $u \in C_{0}^{\infty}(X)$ such that $I(\phi, a) u$ is a continuous function of $a \in S^{m, p}$ for every fixed $(m, p)$. We define an oscillatory integral by

$$
I(\phi, a) u:=\int e^{i \phi(x, \eta, \tau)}\left({ }^{t} L\right)^{k}(a(x, \eta, \tau) u(x)) d \eta d \tau d x,
$$

for $k$ large such that $m-k<-N, p-k<-(N-l)$.

Remark 1.50. We also have

$$
I(\phi, a) u=\lim _{\varepsilon \rightarrow 0} \iiint e^{i \phi(x, \eta, \tau)} a(x, \eta, \tau) \chi(\varepsilon \eta, \varepsilon \tau) u(x) d x d \eta d \tau
$$

for $\chi \in C_{0}^{\infty}\left(\mathbb{R}^{N}\right), \chi(0)=1$.

Next we prove an important result which describes the wave front set of distributions defined by this oscillatory integral.

Proposition 1.51. Assume that $\Gamma$ is a conic cone in $X \times\left(\mathbb{R}^{N-l} \times \mathbb{R}^{l}\right)$ and $\phi(x, \eta, \tau)$ is a multiphase function in $\Gamma$. If $a \in S^{m, p}\left(X \times \mathbb{R}^{N-l} \times \mathbb{R}^{l}\right)$ vanishes near zero section and cone supp $a \subset \Gamma$, then $u \mapsto I(\phi, a) u$ defines a distribution $A(x)$ in $X$. 
Moreover, we have

$$
\begin{aligned}
\mathrm{WF}(A) \subseteq & \left\{\left(x, \phi_{x}\right) \mid(x, \eta, \tau) \in \Gamma, \phi_{\eta}=0, \phi_{\tau}=0\right\} \\
& \cup\left\{\left(x, \phi_{x}\right) \mid(x, \eta, \tau) \in \Gamma, \phi_{\eta}=0, \tau=0\right\} .
\end{aligned}
$$

Proof. Take a point $\left(x_{0}, \xi_{0}\right) \in X \times \mathbb{R}^{n} \backslash 0$, which does not belong to the right hand-side of ([L.43). We shall prove that $\left(x_{0}, \xi_{0}\right) \notin \mathrm{WF}(A)$. Choose a closed conic cone $\Gamma$ which contains cone supp $a$ such that $\left(x_{0}, \xi_{0}\right) \notin F:=\left\{\left(x, \phi_{x}(x, \eta, \tau)\right) \mid(x, \eta, \tau) \in \Gamma ; \phi_{\eta}=0 ; \phi_{\tau}=\right.$ 0 or $\tau=0\}$. We have

$$
\hat{\chi A}(\xi)=\int e^{i(\phi(x, \eta, \tau)-x \xi)} a(x, \eta, \tau) \chi(x) d \eta d \tau d x, \quad \chi \in C_{0}^{\infty}(X)
$$

First, we shall show that there exist $\varepsilon, \delta>0$ and a conic neighbourhood $K$ of $\xi_{0}$ such that

$$
\left|\phi_{x}(x, \eta, \tau)-\xi\right|+|\eta, \tau|\left|\phi_{\eta}(x, \eta, \tau)\right|+|\tau|\left|\phi_{\tau}(x, \eta, \tau)\right| \geq \delta|\eta, \tau, \xi|
$$

whenever $\left|x-x_{0}\right| \leq \varepsilon, \xi \in K$. In fact, since for $(x, \xi)=\left(x_{0}, \xi_{0}\right)$ the left-hand-side of (L.44) is non-zero, the inequality (L.44) holds with $\delta\left(x_{0}, \xi_{0}\right)$ for $x=x_{0}, \xi=\xi_{0},\left(x_{0}, \eta, \tau\right) \in$ $\Gamma$. By the continuity of this function in $(x, \xi)$-variables, there is a conic neighbourhood $B\left(x_{0}, 2 \varepsilon\right) \times K$ of $(x, \xi)$ such that this inequality also holds with $\delta:=\delta\left(x_{0}, \xi_{0}\right) / 2$.

Now take any cut-off function $\chi$ with $\operatorname{supp} \chi \in B\left(x_{0}, \varepsilon\right)$. The operator

$$
L:=-i \frac{\left(\phi_{x}-\xi\right) \partial_{x}+|\eta, \tau|^{2} \phi_{\eta} \partial_{\eta}+|\tau|^{2} \phi_{\tau} \partial_{\tau}}{\left|\phi_{x}-\xi\right|^{2}+|\eta, \tau|^{2}\left|\phi_{\eta}\right|^{2}+|\tau|^{2}\left|\phi_{\tau}\right|^{2}}
$$

satisfies the following properties:

$$
\begin{aligned}
L e^{i(\phi(x . \eta, \tau)-x \xi)} & =e^{i(\phi(x . \eta, \tau)-x \xi \xi}, \\
\left|\left({ }^{t} L\right)^{k}(a \chi)\right| & \leq C_{k}(1+|\eta, \tau, \xi|)^{-k}(1+|\eta, \tau|)^{p}(1+|\tau|)^{m-p} .
\end{aligned}
$$

Hence, take some $k_{0} \in \mathbb{N}: m-k_{0}<-N, p-k_{0}<-(N-l)$, we estimate the terms above as

$$
|\widehat{\chi A}(\xi)| \leq\left|\int e^{i(\phi-x \xi)}\left({ }^{t} L\right)^{k}(a \chi) d \eta d \tau d x\right| \leq C_{k}(1+|\xi|)^{k_{0}-k}
$$

for all $\xi \in K$ and $k>k_{0}$ large enough. This means that $\left(x_{0}, \xi_{0}\right) \notin \operatorname{WF}(A)$.

Theorem 1.52. Let $\phi(x, \eta, \tau)$ be a multiphase function on a conic neighbourhood $\Gamma$ in $X \times\left(\mathbb{R}^{k} \times \mathbb{R}^{l} \backslash 0\right)$. Denote by $\Lambda_{0}, \Lambda_{1}$ the Lagrangian manifolds which are generated by $\phi_{0}, \phi_{1}$ in the following sense:

$$
\begin{aligned}
& \Lambda_{0}=\left\{\left(x, \phi_{x}(x, \eta, \tau)\right) \mid \phi_{\eta}(x, \eta, \tau)=0, \phi_{\tau}(x, \eta, \tau)=0\right\}, \\
& \Lambda_{1}=\left\{\left(x, \phi_{x}(x, \eta, 0) \mid \phi_{\eta}(x, \eta, 0)=0\right\} .\right.
\end{aligned}
$$


If $a(x, \eta, \tau) \in S^{m^{\prime}, p^{\prime}}\left(X \times \mathbb{R}^{k} \times \mathbb{R}^{l}\right)$ with support in $\Gamma$ and $m^{\prime}=m-(k+l) / 2+n / 4, p^{\prime}=$ $p-k / 2+n / 4$, then $\mathrm{WF}(I(a, \phi)) \subseteq \Lambda_{0} \cup \Lambda_{1}$. Moreover, for all $\left(x_{0}, \xi_{0}\right) \in \mathrm{WF}(I(a, \phi))$ the following statements hold:

1. When $\left(x_{0}, \xi_{0}\right) \in \Lambda_{1} \backslash \Lambda_{0}$, there exists a distribution $v(x) \in I^{p}\left(X ; \Lambda_{1}\right)$ such that $\left(x_{0}, \xi_{0}\right) \notin \mathrm{WF}(I(a, \phi)-v)$.

2. When $\left(x_{0}, \xi_{0}\right) \in \Lambda_{0} \backslash \Lambda_{1}$, there exists a distribution $v(x) \in I^{m}\left(X ; \Lambda_{0}\right)$ such that $\left(x_{0}, \xi_{0}\right) \notin \mathrm{WF}(I(a, \phi)-v)$.

3. When $\left(x_{0}, \xi_{0}\right) \in \Lambda_{0} \cap \Lambda_{1}$ and $\psi(x, \eta, \tau)$ is another multiphase function parametrizing the pair $\left(\Lambda_{0}, \Lambda_{1}\right)$ near the point $\left(x_{0}, \xi_{0}\right)$, there exists a function $\tilde{a}(x, \tilde{\eta}, \tilde{\tau}) \in S^{\tilde{m}, \tilde{p}}(X \times$ $\left.\mathbb{R}^{\tilde{k}} \times \mathbb{R}^{\tilde{l}}\right)$ such that $\left(x_{0}, \xi_{0}\right) \notin \mathrm{WF}(I(a, \phi)-I(\tilde{a}, \tilde{\phi}))$.

Proof. We prove the first statement. For $\left(x_{0}, \xi_{0}\right) \in \Lambda_{1} \backslash \Lambda_{0}$, set

$$
F_{x_{0}, \xi_{0}}=\left\{\left(x_{0}, \eta, 0\right) \in \Gamma \mid \phi_{\eta}(x, \eta, 0)=0, \phi_{x}\left(x_{0}, \eta, 0\right)=\xi_{0}\right\} .
$$

The set $F_{x_{0}, \xi_{0}}$ is non-empty and $\phi_{\tau}\left(x_{0}, \eta_{0}, 0\right) \neq 0$ for all $\left(x_{0}, \eta_{0}, 0\right) \in F_{x_{0}, \xi_{0}}$. So we may assume that $\left|\phi_{\tau}\left(x_{0}, \eta, 0\right)\right| \geq \epsilon>0$ on $F_{x_{0}, \xi_{0}}$. Choose a homogeneous smooth function $\chi(x, \eta, \tau)$, which is identical to 1 on $\left\{(x, \eta, \tau)|| \phi_{\tau} \mid \geq \epsilon / 2\right\}$ and vanishes on the set $\left\{(x, \eta, \tau)|| \phi_{\tau}(x, \eta, \tau) \mid \leq \epsilon / 4\right\}$. Define $u(x)=v(x)+w(x)$, where

$$
\begin{aligned}
& w(x)=\int e^{i \phi(x, \eta, \tau)} a(x, \eta, \tau)(1-\chi(x, \eta, \tau)) d \eta d \tau, \\
& v(x)=\int e^{i \phi(x, \eta, 0)}\left(\int e^{i(\phi(x, \eta, \tau)-\phi(x, \eta, 0))} a(x, \eta, \tau) \chi(x, \eta, \tau) d \tau\right) d \eta .
\end{aligned}
$$

We now show that $\left(x_{0}, \xi_{0}\right) \notin \mathrm{WF}(w)$ and $v \in I^{p}\left(X ; \Lambda_{1} \backslash \Lambda_{0}\right)$.

We prove that $\left(x_{0}, \xi_{0}\right) \notin \mathrm{WF}(w)$ by contradiction. Assume that $\left(x_{0}, \xi_{0}\right) \in \mathrm{WF}(w) \subseteq$ $\Lambda_{0} \cup \Lambda_{1}$. Because $\left(x_{0}, \xi_{0}\right) \notin \Lambda_{0}$, we have $\left(x_{0}, \xi_{0}\right) \in \Lambda_{1}$. Hence, there exists $\left(x_{0}, \eta_{0}, 0\right) \in$ $\operatorname{supp} a(1-\chi)$ such that

$$
\xi_{0}=\phi_{x}\left(x_{0}, \eta_{0}, 0\right), \phi_{\eta}\left(x_{0}, \eta_{0}, 0\right)=0
$$

This yields $\left(x_{0}, \eta_{0}, 0\right) \in F_{x_{0}, \xi_{0}}$. Because $(1-\chi)$ vanishes on a neighbourhood of $F_{x_{0}, \xi_{0}}$, we obtain that $a(1-\chi)$ vanishes on a neighbourhood of $\left(x_{0}, \eta_{0}, 0\right)$, which contradicts the fact that $\left(x_{0}, \eta_{0}, 0\right) \in \operatorname{supp} a(1-\chi)$.

In order to show that $v \in I^{p}\left(X ; \Lambda_{0} \backslash \Lambda_{0}\right)$, we need to prove that

$$
b(x, \eta)=\int e^{i(\phi(x, \eta, \tau)-\phi(x, \eta, 0))} a(x, \eta, \tau) \chi(x, \eta, \tau) d \tau \in S^{p^{\prime}}\left(X \times \mathbb{R}^{k}\right) .
$$

By rewriting $\tilde{a}$ as $a \chi$, we may assume that $a \in S^{m^{\prime}, p^{\prime}}$ and $\left|\phi_{\tau}(x, \eta, \tau)\right| \geq \epsilon / 2$ on its support. 
Lemma 1.53. Let $a \in S^{m, p}\left(X \times \mathbb{R}^{k} \times \mathbb{R}^{l}\right)$ vanish near $(\eta, \tau)=0$ and $\phi$ be a multiphase function such that $\left|\phi_{\tau}\right| \geq c$ on the support of a. Define $\psi(x, \eta, \tau)=\phi(x, \eta, \tau)-\phi(x, \eta, 0)$. Then $b(x, \eta):=\int e^{i \psi} a(x, \eta, \tau) d \tau$ belongs to the symbol class $S^{p}\left(X \times \mathbb{R}^{k}\right)$.

To prove this lemma, we use the following facts:

- The function $\psi(x, \eta, \tau) \in S^{1,0}$ on the support of $a$. In fact, $\psi(x, \eta, \tau)=\tau \int_{0}^{1} \phi_{\tau}(x, \eta, s \tau) d s$, where $\int_{0}^{1} \phi_{\tau}(x, \eta, s \tau) d s$ is homogeneous of degree zero in $(\eta, \tau)$. This implies that $\psi \in S^{1,0}$ outside $(\eta, \tau)=0$.

- For all multi-indices $\alpha, \beta, \gamma$, we have

$$
e^{-i \psi(x, \eta, \tau)} \partial_{x}^{\alpha} \partial_{\eta}^{\beta} \partial_{\tau}^{\gamma}\left(e^{i \psi(x, \eta, \tau)} a(x, \eta, \tau)\right) \in S^{m+|\alpha|, p-|\beta|}
$$

Define $L=\frac{\psi_{\tau} \partial_{\tau}}{i\left|\psi_{\tau}\right|^{2}}$, then $L e^{i \psi}=e^{i \psi}$ and $\left({ }^{\prime} L\right)^{r} a \in S^{m-r, p}$. We write

$$
b(x, \eta)=\int e^{i \psi} a(x, \eta, \tau) d \tau=\int e^{i \psi}\left({ }^{\prime} L\right)^{r} a(x, \eta, \tau) d \tau,
$$

The remark above implies that $\partial_{x}^{\alpha} \partial_{\eta}^{\beta} b(x, \eta)=\int e^{i \psi} \tilde{a}(x, \eta, \tau) d \tau$, where $\tilde{a} \in S^{m+|\alpha|-r, p-|\beta|}$. Therefore, for each compact set $K \Subset X$, we estimate

$$
\begin{aligned}
\left|\partial_{x}^{\alpha} \partial_{\eta}^{\beta} b(x, \eta)\right| & \leq C_{K, \alpha, \beta, r} \int\langle\eta, \tau\rangle^{p-|\beta|}\langle\tau\rangle^{m+|\alpha|-r-(p-|\beta|)} d \eta \\
& \leq C\langle\eta\rangle^{p-|\beta|}\langle\tau\rangle^{|p-| \beta||+m+|\alpha|-r-(p-|\beta|)} d \eta \leq C\langle\eta\rangle^{p-|\beta|}
\end{aligned}
$$

if we choose $r$ large enough, for instance $|p-| \beta||+m+|\alpha|+|\beta|-p<r-l$. This means that $b \in S^{p}\left(X \times \mathbb{R}^{k}\right)$, and the lemma is proved.

Apply this lemma, we conclude that $v \in I^{p}\left(X ; \Lambda_{1} \backslash \Lambda_{0}\right)$. This proves the first statement of the theorem.

The second statement is proved similarly. Define

$$
F_{x_{0}, \xi_{0}}=\left\{\left(x_{0}, \eta, \tau\right) \mid \phi_{x}\left(x_{0}, \eta, \tau\right)=\xi_{0}, \phi_{\eta}\left(x_{0}, \eta, \tau\right)=0, \phi_{\tau}(x, \eta, \tau)=0\right\}
$$

Because $\left(x_{0}, \xi_{0}\right) \in \Lambda_{0} \backslash \Lambda_{1}$, the set $F_{x_{0}, \xi_{0}}$ is non-empty and $\tau \neq 0$ for all $\left(x_{0}, \eta, \tau\right) \in$ $F_{x_{0}, \xi_{0}}$. Hence, we may assume that $|\tau| \geq \epsilon|\eta, \tau|$ for all $\left(x_{0}, \eta, \tau\right) \in F_{x_{0}, \xi_{0}}$. Take a smooth, homogeneous function $\chi(\eta, \tau)$ such that $\chi$ is identical to 1 on $|\tau| \geq \epsilon|\eta, \tau| / 2$, vanishes on the set $|\tau| \geq \epsilon|\tau, \eta| / 4$. Then $(1-\chi)$ vanishes on a neighbourhood of $F_{x_{0}, \eta_{0}}$. By setting $v(x)=I(a \chi, \phi)$ and $w(x)=I(a(1-\chi), \phi)$, we get $u(x)=v(x)+w(x)$. 
We now show that $\left(x_{0}, \xi_{0}\right) \notin \mathrm{WF}(w)$ and $v \in I^{m}\left(X ; \Lambda_{1}\right)$. Indeed, if $\left(x_{0}, \xi_{0}\right) \in \mathrm{WF}(w) \subseteq$ $\Lambda_{0} \cup \Lambda_{1}$, this yields $\left(x_{0}, \xi_{0}\right) \in \Lambda_{0}$. Thus, there exists a point $\left(x_{0}, \eta_{0}, \tau_{0}\right) \in \operatorname{supp} a(1-\chi)$ such that $\xi_{0}=\phi_{x}\left(x_{0}, \eta_{0}, \tau_{0}\right), \phi_{\eta}=0, \phi_{\tau}=0$. Thus $\left(x_{0}, \eta_{0}, \tau_{0}\right) \in F_{x_{0}, \xi_{0}}$. This implies that $(1-\chi)$ vanishes on a neighbourhood of this point, which contradicts the assumption $\left(x_{0}, \eta_{0}, \tau_{0}\right) \in \operatorname{supp} a(1-\chi)$.

Moreover, to prove that $v \in I^{m}\left(X ; \Lambda_{0} \backslash \Lambda_{1}\right)$, we note that on the support of $a \chi$, we have $|\tau| \geq \epsilon|\eta, \tau|$. Combining this with the fact that $a \in S^{m^{\prime}, p^{\prime}}$, we conclude that $a \chi \in$ $S^{m^{\prime}}\left(X \times \mathbb{R}^{k+l}\right)$. This shows that $v=I(a \chi, \phi)$ belongs to the class $I^{m}\left(X ; \Lambda_{0} \backslash \Lambda_{1}\right)$.

This theorem has the following immediate consequences:

Corollary 1.54. Let $\phi(x, \eta, \tau)$ be a multiphase function and $a \in S^{m-(k+l) / 2+n / 4, p-k / 2+n / 4}(X \times$ $\left.\mathbb{R}^{k} \times \mathbb{R}^{l}\right)$. Define $u(x)=I(a, \phi)$. Let $A \in \Psi^{r}(X)$ be a properly supported pseudo-differential operator of order $r$ on $X$.

a) If $\mathrm{WF}^{\prime}(A) \cap \Lambda_{0}=\emptyset$, then $A u \in I^{r+p}\left(X ; \Lambda_{1} \backslash \Lambda_{0}\right)$. If $\mathrm{WF}^{\prime}(A) \cap \Lambda_{1}=\emptyset$, then $A u \in I^{r+m}\left(X ; \Lambda_{0} \backslash \Lambda_{1}\right)$.

b) If $W F(u) \subseteq \Lambda_{0} \backslash \Lambda_{1}$, then $u \in I^{m}\left(X ; \Lambda_{0} \backslash \Lambda_{1}\right)$. Similarly, if $\operatorname{WF}(u) \subseteq \Lambda_{1} \backslash \Lambda_{0}$, then $u \in I^{p}\left(X ; \Lambda_{1} \backslash \Lambda_{0}\right)$.

Definition 1.55. Let $\Lambda_{0}, \Lambda_{1}$ be an intersecting pair of Lagrangian manifolds in $T^{*} X \backslash 0$. Assume that $H(\eta)$ and $\phi(x, \eta, \tau)$ are homogeneous functions such that $\phi(x, \eta, \tau+H(\eta))$ is a multiphase function on $X \times\left(\mathbb{R}^{k} \times \mathbb{R}^{l} \backslash 0\right)$ parametrizing the pair $\Lambda_{0}, \Lambda_{1}$. Let $\mathbb{J}^{m, p}\left(\Lambda_{0}, \Lambda_{1}\right)$, where $l=\operatorname{codim}_{\Lambda_{0}}\left(\Lambda_{0} \cap \Lambda_{1}\right)$, denote the set of all distribution $u$ can be defined as the oscillatory integral

$$
u(x)=(2 \pi)^{-(k+l) / 2-n / 4} \int e^{i \phi(x, \eta, \tau)} a(x, \eta, \tau) d \eta d \tau,
$$

where the amplitude function $a \in S^{m^{\prime}, p^{\prime}}$ is of the form

$$
a(x, \eta, \tau)=\tilde{a}\left(x, \frac{\eta}{\langle\tau-H(\eta)\rangle}, \tau-H(\eta)\right),
$$

for some $\tilde{a}(x, \tau, \eta) \in S^{p^{\prime}}\left(X \times \mathbb{R}^{k}\right) \hat{\otimes}_{C^{\infty}(X)} S^{m^{\prime}}\left(X \times \mathbb{R}^{l}\right), m^{\prime}=m-(k+l) / 2+n / 4, p^{\prime}=$ $p-k / 2+n / 4$.

We now refine the assumptions by requiring that $\tilde{a} \in S_{\mathrm{cl}}^{p^{\prime}}\left(X \times \mathbb{R}^{k}\right) \hat{\otimes}_{C^{\infty}(X)} S_{\mathrm{cl}}^{m^{\prime}}\left(X \times \mathbb{R}^{l}\right)$, where $S_{\mathrm{cl}}^{p}\left(X \times \mathbb{R}^{k}\right)$ is the space of classical symbols. 
Remark 1.56. If $a(x, \eta, \tau) \in S_{\mathrm{cl}}^{m}\left(X \times \mathbb{R}^{k+l}\right)$ and $\tilde{a}(x, \tilde{\eta}, \tau):=a(x, \tilde{\eta}\langle\tau\rangle, \tau)$, then $\tilde{a} \in S_{\mathrm{cl}}^{m}(X \times$ $\left.\mathbb{R}^{k}\right) \hat{\otimes}_{C^{\infty}(X)} S_{\mathrm{cl}}^{m}\left(X \times \mathbb{R}^{l}\right)$. The leading homogeneous parts $\tilde{a}_{0}, \tilde{a}_{1}, \tilde{a}_{00}$ of $\tilde{a}$ in $\tilde{\eta}$, in $\tau$, and in $(\tilde{\eta}, \tau)$, are given as follows:

$$
\begin{gathered}
\tilde{a}_{0}(x, \tilde{\eta}, \tau)=\lim _{t \rightarrow \infty} t^{-m} a(x, t \tilde{\eta}\langle\tau\rangle, \tau)=\langle\tau\rangle^{m} a_{0}(x, \tilde{\eta}, 0), \\
\tilde{a}_{1}(x, \tilde{\eta}, \tau)=|\tau|^{m} a_{0}\left(x, \tilde{\eta}, \frac{\tau}{|\tau|}\right), \\
\tilde{a}_{00}(x, \tilde{\eta}, \tau)=|\tau|^{m} a_{0}(x, \tilde{\eta}, 0) .
\end{gathered}
$$

In general, an intersecting pair may have no global multiphase function. In this case, we use the micro-local definition of this space as follows:

Definition 1.57. Let $\Lambda_{0}, \Lambda_{1}$ be an intersecting pair of closed conic Lagrangian manifolds in $T^{*} X \backslash 0$. We write $J^{m, p}\left(X ; \Lambda_{0}, \Lambda_{1}\right)$ for the space of all distribution $u \in D^{\prime}(X)$ such that $\mathrm{WF}(u) \subseteq \Lambda_{0} \cup \Lambda_{1}$ and for all $(x, \xi) \in \mathrm{WF}(u)$, the distribution $u$ can be micro-locally written as the oscillatory integral $u=I(a, \phi)$, for some local multiphase function $\phi$ parametrizing the pair $\Lambda_{0}, \Lambda_{1}$ near $\left(x_{0}, \xi_{0}\right)$ and an amplitude function $a \in S^{m-(k+l) / 2+n / 4, p-k / 2+n / 4}\left(X \times \mathbb{R}^{k} \times\right.$ $\mathbb{R}^{l}$ ).

Set $\mathbb{J}\left(\Lambda_{0}, \Lambda_{1}\right)=\cup_{m, p} \mathbb{J}^{m, p}\left(\Lambda_{0}, \Lambda_{1}\right)$. Now we prove that this class is invariant under action of Fourier integral operators.

Theorem 1.58. Suppose that a homogeneous canonical relation $C$ from $T^{*} Y \backslash 0$ to $T^{*} X \backslash 0$ and a pair of Lagrangian submanifolds $\left(\Lambda_{0}, \Lambda_{1}\right)$ of $T^{*} Y \backslash 0$ are satisfied the assumption in Theorem L.28. Let $A \in I^{q}(X \times Y ; C)$ be a properly supported operator and $u \in \mathbb{J}^{m, p}\left(Y ; \Lambda_{0}, \Lambda_{1}\right)$. Then $A u \in \mathbb{J}^{q+m, q+p}\left(X ; C \circ \Lambda_{0}, C \circ \Lambda_{1}\right)$.

Proof. We follow the method used to prove the composition of Lagrangian distributions. First, we assume that $X \subseteq \mathbb{R}^{n_{X}}, Y \subseteq \mathbb{R}^{n_{Y}}$ and

$$
\begin{aligned}
A(x, y) & =(2 \pi)^{-\left(n_{X}+n_{Y}+2 N_{\xi}\right) / 4} \int e^{i \Phi(x, y, \xi)} a(x, y, \xi) d \xi, \\
u(y) & =(2 \pi)^{-n_{Y} / 4-\left(n_{\eta}+n_{\tau}\right) / 2} \int e^{\varphi(y, \eta, \tau)} b(y, \eta, \tau) d \eta d \tau,
\end{aligned}
$$

where $\Phi(x, y, \xi)$ is a regular phase function in a neighbourhood $\Gamma_{1} \subseteq X \times Y \times\left(\mathbb{R}^{N_{\xi}} \backslash 0\right)$ of $\left(x_{0}, y_{0}, \xi_{0}\right)$ parametrizing $C, \varphi(y, \eta, \tau)$ is a regular multiphase function in a neighbourhood $\Gamma_{2} \subseteq Y \times\left(\mathbb{R}^{n_{\eta}} \backslash 0\right) \times \mathbb{R}^{n_{\tau}}$ of $\left(y_{0}, \eta_{0}, \tau_{0}\right)$ parametrizing $\left(\Lambda_{0}, \Lambda_{1}\right), a \in S^{q^{\prime}}, b \in S^{m^{\prime}, p^{\prime}}$ have order $q^{\prime}=q+\left(n_{X}+n_{Y}\right) / 4-n_{\xi} / 2, m^{\prime}=m+n_{Y} / 4-\left(n_{\eta}+n_{\tau}\right) / 2, p+n_{Y} / 4-n_{\eta} / 2$ and support in 
a closed cone of $\Gamma_{1}, \Gamma_{2}$, respectively. If $a \in S^{-\infty}, b \in S^{-\infty}$, then

$$
A u(x)=(2 \pi)^{-N} \int e^{i \psi(x, y, \xi, \eta, \tau)} a(x, y, \xi) b(y, \eta, \tau) d y d \xi d \eta d \tau
$$

where $N=n_{X} / 4+\left(n_{Y}+n_{\xi}+n_{\eta}+n_{\tau}\right) / 2$ and $\psi(x, y, \xi, \eta, \tau)=\Phi(x, y, \xi)+\varphi(y, \eta, \tau)$. By Theorem 1.28 , we know that $(\psi, H)$ is a multiphase function parametrizing $\left(C \circ \Lambda_{0}, C \circ \Lambda_{1}\right)$. Since $\Phi$ and $\varphi$ are phase functions, there are positive constants $c_{1}, c_{2}$ such that $c_{1}|\xi|<$ $|\eta, \tau|<c_{2}|\xi|$ if $(x, y, \xi) \in \operatorname{supp} a,(y, \eta, \tau) \in \operatorname{supp} b$ and

$$
\Phi_{\xi}=0, \varphi_{\eta}=0, \varphi_{\tau}=0, \Phi_{y}+\varphi_{y}=0
$$

Note that in the part $\{|\eta| \leq c|\tau-H(\eta)|\}, u \in I^{m}\left(Y ; \Lambda_{0}\right)$ is a Lagrangian distribution, then we obtain $A u \in I^{m+q}\left(C \circ \Lambda_{0}\right)$ by the classical theory. Hence we only need to consider the region $\{|\eta| \geq c|\tau-H(\eta)|\}$. Let $\chi(\xi, \eta, \tau)$ be a homogeneous function of degree 0 satisfying

$$
\chi(\xi, \eta, \tau)=\left\{\begin{array}{ll}
1 & ,(\xi, \eta, \tau) \in\left\{c_{1} / 2|\xi|<|\eta, \tau|<c_{2} / 2|\xi|\right\} \\
0 & ,(\xi, \eta, \tau) \notin\left\{c_{1} / 3|\xi|<|\eta, \tau|<c_{2} / 3|\xi|\right\}
\end{array} .\right.
$$

Set $c(x, y, \xi, \eta, \tau)=\chi(\xi, \eta, \tau) a(x, y, \xi) b(y, \eta, \tau)$ and $r(x, y, \xi, \eta, \tau)=(1-\chi) a b$. In the support of $r$ we have

$$
\left|\partial_{y} \psi(x, y, \xi, \eta, \tau)\right| \geq c|\xi, \eta, \tau|
$$

Therefore, the distribution corresponding to $r$ is a $C^{\infty}$ function on $X$, which depends continuously on $a$ and $b$.

We have $c(x, y, \xi, \eta, \tau) \in S^{m+q+\left(n_{X}+2 n_{Y}\right) 2-\left(n_{\xi}+n_{\eta}+n_{\tau}\right) / 4, p+q+\left(n_{X}+2 n_{Y}\right) 2-\left(n_{\xi}+n_{\eta}\right) / 4}(X \times Y)$.

Changing variables $y \mapsto \theta=y\left(|\xi|^{2}+|\eta|^{2}\right)^{1 / 2}$, we have

$$
|\xi, \eta|^{-n_{y}} c(x, \theta /|\xi, \eta|, \xi, \eta, \tau) \in S^{\left.q+m+n_{X} / 4-2\left(n_{Y}+n_{\xi}+n_{\eta}\right)\right) / 2, p+n_{X} / 4-\left(n_{\tau}+n_{Y}+n_{\xi}\right) / 2}
$$

This shows that $A u \in \mathbb{J}^{q+m, q+p}\left(C \circ \Lambda_{0}, C \circ \Lambda_{1}\right)$.

Theorem 1.59. Let $\phi$ be a regular multiphase function parametrizing an intersecting pair of Lagrangian manifolds $\Lambda_{0}, \Lambda_{1}$. Then the space $\mathbb{J}^{m, p}\left(\Lambda_{0}, \Lambda_{1}\right)$ coincides with the space of paired Lagrangian distributions $I^{m, p}\left(\Lambda_{0}, \Lambda_{1}\right)$.

Proof. Because both spaces $\mathbb{J}^{m, p}\left(\Lambda_{0}, \Lambda_{1}\right)$ and $I^{m, p}\left(\Lambda_{0}, \Lambda_{1}\right)$ are invariant under action of Fourier integer operators of order zero, we only need to prove for the model pair $\left(\tilde{\Lambda}_{0}, \tilde{\Lambda}_{1}\right)$. Recall that $I^{m, p}\left(\Lambda_{0}, \Lambda_{1}\right)$ are defined by oscillatory integral with respect to the model multiphase $\phi(x, \eta, \tau)=x^{\prime} \eta+x^{\prime \prime} \tau$ and the usual functional spaces $S^{m-n / 4, p-n / 4+l / 2}\left(X \times \mathbb{R}^{n-l} \times \mathbb{R}^{l}\right)$. 
First of all, it is obvious that $I^{m, p}\left(\Lambda_{0}, \Lambda_{0}\right) \subseteq \mathbb{J}^{m, p}\left(\Lambda_{0}, \Lambda_{1}\right)$. It is sufficient to prove the inverse conclusion.

Given $u \in \mathbb{J}^{m, p}\left(\Lambda_{0}, \Lambda_{1}\right)$. By definition, $u(x)$ is defined as the oscillatory integral of a multiphase function $\phi(x, \eta, \tau)$ and an amplitude function $a(x, \eta, \tau) \in S^{m+n / 4-(k+l) / 2, p+n / 4-k / 2}$. Using Lemma 1.60, we can rewrite

$$
u(x)=\int e^{i x \xi} b(x, \xi) d \xi, \quad a \in S^{m-n / 4, p-n / 4+l / 2} .
$$

This implies that $u \in I^{m, p}\left(\Lambda_{0}, \Lambda_{1}\right)$.

The proof is completed by applying the following lemma, which states that each regular multiphase function can be reduced to a model multiphase function:

Lemma 1.60. Consider a model pair of Lagrangian submanifolds in $T^{*} \mathbb{R}^{n}$ :

$$
\Lambda_{0}=\{(x, \xi) \mid x=0\}, \Lambda_{1}=\left\{(x, \xi) \mid x^{\prime}=0, \xi^{\prime \prime}=0\right\} ; x^{\prime}=\left(x_{1}, \ldots, x_{n-l}\right), x^{\prime \prime}=\left(x_{n-l+1, \ldots, x_{n}}\right) .
$$

Assume that

$$
u(x)=\int e^{i \varphi(x, \eta, \tau)} a(x, \eta, \tau) d \eta d \tau,
$$

where $a \in S^{m+n / 4-(k+l) / 2, p+n / 4-k / 2}$ and $(\varphi, H)$ is a regular multiphase function parametrizing the pair $\left(\Lambda_{0}, \Lambda_{1}\right)$. Then there exists an amplitude function $\tilde{a}\left(x, \xi^{\prime}, \xi^{\prime \prime}\right) \in S^{m-n / 4, p-n / 4+l / 2}$ such that

$$
u(x)=\int e^{i x \xi} \tilde{a}\left(x, \xi^{\prime}, \xi^{\prime \prime}\right) d \xi
$$

Proof. Step 1. Without loss of generality, one takes $H=0$. In fact, if one defines

$$
\phi(x, \eta, \tau)=\varphi(x, \eta, \tau+H(\eta)), \tilde{a}(x, \eta, \tau)=a(x, \eta, \tau+H(\eta)),
$$

then $(\phi, H=0)$ is a multiphase function which parametrizes $\Lambda_{0}, \Lambda_{1}$. Hence, $u$ can be represented in the form

$$
\int e^{i \phi(x, \eta, \tau)} \tilde{a}(x, \eta, \tau) d \eta d \tau, \tilde{a} \in S^{m^{\prime}, p^{\prime}}
$$

Step 2. Reduce the number of $\eta$-variables and $\phi(x, \eta, 0)=x^{\prime} \eta$. One can reduce the number of $\eta$-variables until $\varphi_{\eta \eta}=0$ at the point $\left(x_{0}, \eta_{0}, 0\right)$. Since $\varphi_{1}(x, \eta)=\left.\varphi(x, \eta, \tau)\right|_{\tau=0}$ parametrizes $\Lambda_{1}$, we have $\varphi_{\eta}(x, \eta, 0)=0$ is equivalent to $x^{\prime}=0, \varphi_{x^{\prime \prime}}(x, \eta, 0)=0$. Indeed, assume that $\operatorname{rank}\left(\varphi_{1 \eta \eta}\right)(x, \eta, 0)=r$, by linear transformation in $\eta$, this leads to

$$
\begin{aligned}
\eta=\left(\eta^{\prime}, \eta^{\prime \prime}\right), \eta^{\prime}=( & \left.\eta_{1}, \ldots, \eta_{k-r}\right), \eta^{\prime \prime}=\left(\eta_{k-r+1} \ldots, \eta_{k}\right), \\
d_{\eta^{\prime}}^{2} \varphi\left(x_{0}, \eta_{0}, 0\right) & =0, d_{\eta^{\prime}} d_{\eta^{\prime \prime}} \varphi\left(x_{0}, \eta_{0}, 0\right)=0, \\
\left.d_{\eta^{\prime \prime} \eta^{\prime \prime}}^{2} \varphi\left(x_{0}, \eta_{0}, \tau\right)\right) & =r \text { for } \tau /|\eta| \text { small enough. }
\end{aligned}
$$


As $\left.d_{\eta^{\prime \prime} \eta^{\prime \prime}}^{2} \varphi\left(x_{0}, \eta_{0}, \tau\right)\right)$ is non-degenerate, the equation $d_{\eta^{\prime \prime}} \varphi(x, \eta, \tau)=0$ is solved by $\eta^{\prime \prime}=$ $g\left(x, \eta^{\prime}, \tau\right)$. Applying the stationary phase method in $\eta^{\prime \prime}$-variables, one obtains

$$
\int a(x, \eta, \tau) e^{i \phi(x . \eta, \tau)} d \eta^{\prime \prime}=e^{i \phi\left(x, \eta^{\prime}, g\left(x, \eta^{\prime}, \tau\right), \tau\right)} b\left(x, \eta^{\prime}, \tau\right),
$$

where the function $b$ has an asymptotic expansion such that

$$
b\left(x, \eta^{\prime}, \tau\right)-a\left(x, \eta^{\prime}, g\left(x, \eta^{\prime}, \tau\right), \tau\right) \in S^{m^{\prime}+r / 2-1, p^{\prime}+r / 2-1} .
$$

Consequently, one obtains

$$
b \in S^{m+n / 4-(k+l-r) / 2, p+n / 4-(k-r) / 2} .
$$

So one can assume that $k=n-l, d_{\eta \eta}^{2} \varphi(x, \eta, \tau)=0$ in an open conic neighbourhood $V$ of $\left(x_{0}, \eta_{0}, \tau_{0}\right)$ and that $a \in S^{m-n / 4, p-n / 4+l / 2}$ has support in $V$.

Step 3. By Proposition 1 .34, two multiphase functions $\phi(x, \eta, \tau)$ and $x^{\prime} \xi^{\prime}+x^{\prime \prime} \xi^{\prime \prime}$ are locally equivalent at $\left(x_{0}, \eta_{0}, 0\right)$ and $\left(x_{0}, \xi_{0}\right)$ with $\xi_{0}=\phi_{x}\left(x_{0}, \eta_{0}, 0\right)$. Hence, there is a diffeomorphism transformation $(x, \xi) \rightarrow(x, \eta(x, \xi), \tau(x, \xi)$ such that $\phi(x, \eta, \tau)=\xi$ on the set $|\tau| /|\eta| \leq \varepsilon$. On this set, the symbol $a(x, \eta, \tau)$ behaves like a classical symbol in $S^{m^{\prime}}$, this part contributes to $I^{m}\left(\Lambda_{0}\right)$. So we assume that the amplitude function has cone supp $a$ in a small conic neighbourhood of $\left(x_{0}, \eta_{0}, 0\right)$. Using the change of variables above, one can rewrite

$$
u(x)=\int e^{i x \xi} \tilde{a}(x, \xi) d \xi
$$

where $\tilde{a}=a(x, \eta(x, \xi), \tau(x, \xi))\left|D(\eta, \tau) / D\left(\xi^{\prime}, \xi^{\prime \prime}\right)\right| \in S^{m^{\prime}, p^{\prime}}$ by Corollary 1.45. The lemma is proved.

\subsubsection{Representation of paired Lagrangian distributions via degen- erate multiphase functions}

Although each intersecting pair of Lagrangian manifolds can always be parametrized by a non-degenerate multiphase function, it is convenient to consider the representation of paired Lagrangian distributions by oscillatory integrals of clean multiphase functions and amplitude functions. This representation appears naturally when a Fourier integral operator composes cleanly with a paired Lagrangian distribution. Now we introduce a new result which states that distributions defined by clean multiphase function indeed belong to the class of paired Lagrangian distributions. 
Theorem 1.61. Let $\phi$ be a clean multiphase function with excess $e$ in a conic neighbourhood of $\left(x_{0}, \eta_{0}, 0\right)$. If $a \in S^{m+n / 4-N / 2, p+n / 4-(N-l) / 2}\left(X \times \mathbb{R}^{N-l} \times \mathbb{R}^{l}\right)$, then the oscillatory integral $I(\phi, a)$ defines a distribution belonging to $I^{m+e / 2, p+e / 2}\left(\Lambda_{0}, \Lambda_{1}\right)$, where $\Lambda_{0}=\left\{\left(x, \phi_{x}\right) \mid \phi_{\eta}=\right.$ $\left.0, \phi_{\tau}=0\right\}, \Lambda_{1}=\left\{\left(x, \phi_{x}\right) \mid \phi_{\eta}=0, \tau=0\right\}$.

Proof. First, we assume that there is a splitting of fibre variables $(\eta, \tau) \rightarrow\left(\eta^{\prime}, \eta^{\prime \prime}, \tau\right)$ satisfying the following essential condition

$$
\partial_{\eta^{\prime \prime}} \phi(x, \eta, \tau)=0
$$

By rewriting the distribution $u$ in the form

$$
u(x)=\int_{\omega} d \omega \int e^{i \phi\left(x, \eta^{\prime},\left|\eta^{\prime}, \tau\right| \omega, \tau\right)}\left|\eta^{\prime}, \tau\right|^{e} a\left(x, \eta^{\prime},\left|\eta^{\prime}, \tau\right| \omega, \tau\right) d \eta^{\prime} d \tau
$$

we derive the conclusion of the theorem.

Indeed, define $\psi\left(x, \eta^{\prime}, \tau\right)=\phi\left(x, \eta^{\prime},\left|\eta^{\prime}, \tau\right| \omega, \tau\right)$ for fixed $\omega$. We shall show that $\psi$ is a new regular multiphase function parametrizing the pair of Lagrangian submanifold $\left(\Lambda_{0}, \Lambda_{1}\right)$. Because $d \phi_{\eta^{\prime \prime}}=0$, we have

$$
\operatorname{rank}\left(\begin{array}{cc}
\psi_{x \eta^{\prime}} & \psi_{\eta^{\prime} \eta^{\prime}} \\
\psi_{x \tau} & \psi_{\eta^{\prime} \tau}
\end{array}\right)\left(x_{0}, \eta_{0}^{\prime}, 0\right)=\operatorname{rank}\left(\begin{array}{ccc}
\phi_{x \eta} & \phi_{\eta^{\prime} \eta^{\prime}} & 0 \\
0 & 0 & 0 \\
\phi_{x \tau} & \phi_{\eta^{\prime} \tau} & 0
\end{array}\right)\left(x_{0}, \eta_{0}, 0\right)=N-e .
$$

This means that the function $\psi\left(x, \eta^{\prime}, \tau\right)$ is a regular multiphase function. Just by a simple calculation, we see that $\psi$ parametrizes the pair $\left(\Lambda_{0}, \Lambda_{1}\right)$ near $\left(x_{0}, \xi_{0}\right)$. For a fixed $\omega$ the distribution $I(\omega)=\int e^{i \psi\left(x, \eta^{\prime}, \tau\right)}\left|\eta^{\prime}, \tau\right|^{e} a\left(x, \eta^{\prime},\left|\eta^{\prime}, \tau\right| \omega, \tau\right) d \eta^{\prime} d \tau$ belongs to $I^{m+e / 2, p+e / 2}$, so does the integral $u(x)=\int_{\mathbb{R}^{e}} I(\omega) d \omega$. This proves the claim.

Now we shall complete the proof of this theorem by showing that after a suitable change of fibre variables, the condition (11.49) holds. In fact, splitting $\eta=\left(\eta^{\prime}, \eta^{\prime \prime}\right)$ such that at $\left(x_{0}, \eta_{0}, 0\right)$ we have

$$
\operatorname{rank}\left(\begin{array}{cccc}
\phi_{x \eta^{\prime}} & \phi_{\eta^{\prime} \eta^{\prime}} & \phi_{\eta^{\prime \prime} \eta^{\prime}} & \phi_{\tau \eta^{\prime}} \\
\phi_{x \eta^{\prime \prime}} & \phi_{\eta^{\prime} \eta^{\prime \prime}} & \phi_{\eta^{\prime \prime} \eta^{\prime \prime}} & \phi_{\tau \eta^{\prime \prime}} \\
\phi_{x \tau} & \phi_{\eta^{\prime} \tau} & \phi_{\eta^{\prime \prime} \tau} & \phi_{\tau \tau}
\end{array}\right)=\operatorname{rank}\left(\begin{array}{cccc}
\phi_{x \eta^{\prime}} & \phi_{\eta^{\prime} \eta^{\prime}} & 0 & \phi_{\tau \eta^{\prime}} \\
0 & 0 & 0 & \phi_{\tau \eta^{\prime \prime}} \\
\phi_{x \tau} & \phi_{\eta^{\prime} \tau} & \phi_{\eta^{\prime \prime} \tau} & \phi_{\tau \tau}
\end{array}\right) .
$$

Using procedure of eliminating excess for $\phi_{1}(x, \eta)=\phi(x, \eta, 0)$, we can assume that $\phi_{\eta^{\prime \prime}}(x, \eta, 0)=0$ in some neighbourhood of $\left(x_{0}, \eta_{0}\right)$. Since $\phi_{\eta^{\prime \prime}}$ vanishes on the set $\left\{\phi_{\tau^{\prime}}=\right.$ $\left.0, \phi_{\tau}=0\right\}$, there are matrices $p, q$ whose elements are smoothly homogeneous functions of degree zero with the property $\phi_{\eta^{\prime \prime}}=\phi_{\eta^{\prime}} p+\phi_{\tau} q$. The function $\phi_{\eta^{\prime \prime}}(x, \eta, \tau)-$ 
$\phi_{\eta^{\prime}}(x, \eta, \tau) p(x, \eta, \tau)$ vanishes on the cleanly intersecting pair $\left\{(x, \eta, \tau) \mid \phi_{\eta^{\prime}}(x, \eta, \tau)=0, \phi_{\tau}=\right.$ $0\}$ and $\left\{(x, \eta, \tau) \mid \phi_{\eta^{\prime}}=0, \tau=0\right\}$. Hence, there exist smooth functions $c(x, \eta, \tau), d(x, \eta, \tau)$ satisfying

$$
\phi_{\eta^{\prime \prime}}(x, \eta, \tau)-\phi_{\eta^{\prime}}(x, \eta, \tau) p(x, \eta, \tau)=\phi_{\eta^{\prime}}(x, \eta, \tau) c(x, \eta, \tau)+\tau \phi_{\tau}(x, \eta, \tau) d(x, \eta, \tau) .
$$

This implies

$$
\phi_{\eta^{\prime \prime}}(x, \eta, \tau)=\phi_{\eta^{\prime}}(x, \eta, \tau) f(x, \eta, \tau)+\phi_{\tau}(x, \eta, \tau) g(x, \eta, \tau),
$$

where $f=p+c$, and $g=\tau d$ are matrices of smoothly homogeneous functions, and $g(x, \eta, 0)=0$. This fact leads to the existence of changing of coordinates $(x, \eta, \tau) \mapsto$ $\left(x, \eta^{\prime}+\tilde{f}, \eta^{\prime \prime}, \tau+\tilde{g}\right)$ satisfying $\tilde{g}(x, \eta, 0)=0$. Let us define $\tilde{\phi}(x, \eta, \tau)=\phi\left(x, \eta^{\prime}+\tilde{f}, \eta^{\prime \prime}, \tau+\tilde{g}\right)$, where $\tilde{f}, \tilde{g}$ are determined just as in Remark L.12. Then $\tilde{\phi}$ and $\phi$ are locally equivalent in some conic neighbourhood of $\left(x_{0}, \eta_{0}, 0\right)$ and $\tilde{\phi}_{\eta^{\prime \prime}}=0$. So we may assume that $\phi_{\eta^{\prime \prime}}(x, \eta, \tau)=$ 0 . The theorem is proved.

Theorem [.6] has the following generalization.

Theorem 1.62. Let $\phi$ be a degenerate multiphase function with excesses $\left(e_{0}, e_{1}, e\right)$ in an open conic neighbourhood $\Gamma \subseteq X \times\left(\mathbb{R}^{k} \times \mathbb{R}^{l} \backslash 0\right)$ of $\left(x_{0}, \eta_{0}, 0\right)$. Then $\Lambda_{0}=\left\{\left(x, \phi_{x}\right) \mid \phi_{\theta}(x, \theta)=\right.$ $0\}$ and $\Lambda_{1}=\left\{\left(x, \phi_{x}\right) \mid \phi_{\eta}(x, \eta, \tau)=0, \tau=0\right\}$ intersect cleanly near $\left(x_{0}, \xi_{0}\right)$ with $\xi_{0}=$ $\phi_{x}\left(x_{0}, \eta_{0}, 0\right)$, and the intersection $\Lambda_{0} \cap \Lambda_{1}$ is an isotropic submanifold of dimension

$$
\left(n-l-e_{0}-e_{1}+2 e\right)
$$

Moreover, if a $\in S^{m+n / 4-(k+l) / 2, p+n / 4-k / 2}(\Gamma)$ has support in a small conic neighbourhood of $\left(x_{0}, \eta_{0}, 0\right)$, then the distribution $I(\phi, a)$ belongs to the space $I^{m+e_{0} / 2, p+e_{1} / 2}\left(\Lambda_{0}, \Lambda_{1}\right)$.

Proof. First we make an additional condition that $e_{0}=e$. By Proposition 11.37, we assume that $\eta=\left(\eta^{\prime}, \eta^{\prime \prime}\right), \tau=\left(\tau^{\prime}, \tau^{\prime \prime}\right)$ such that $\phi_{\omega}(x, \eta, \tau)=0$, where $\omega=\left(\eta^{\prime \prime}, \tau^{\prime \prime}\right)$. Using a change of variables $\eta^{\prime \prime}=\omega_{1}\left|\eta^{\prime}, \tau^{\prime}\right|, \tau^{\prime \prime}=\omega_{2}\left|\eta^{\prime}, \tau^{\prime}\right|$, then $a=a\left(x, \eta^{\prime}, \omega_{1}\left|\eta^{\prime}, \tau^{\prime}\right|, \tau^{\prime}, \omega_{2}\left|\eta^{\prime}, \tau^{\prime}\right|\right)$. Since $a$ has support in some small conic neighbourhood of $\left(x_{0}, \eta_{0}, 0\right), \eta_{0}^{\prime} \neq 0$, we may assume that $|\omega|=\left|\eta^{\prime \prime}, \tau^{\prime \prime}\right| /\left|\eta^{\prime}, \tau^{\prime}\right|$ is bounded on the support of $a$. For fixed $\omega$, a simple calculation shows that

$$
a \in S^{m^{\prime}, p^{\prime}}\left(X \times \mathbb{R}^{k-\left(e-e_{1}\right)} \times \mathbb{R}^{l-e_{1}}\right) .
$$

Rewriting $I(a, \phi)$ in new variables, we have

$$
I(a, \phi)=\int_{\omega}\left(\int e^{i \phi\left(x, \eta^{\prime},\left|\eta^{\prime}, \tau^{\prime}\right| \omega_{1}, \tau^{\prime},\left|\eta^{\prime}, \tau^{\prime}\right| \omega_{2}\right)}\left|\eta^{\prime}, \tau^{\prime}\right|^{e} a\left(x, \eta^{\prime},\left|\eta^{\prime}, \eta^{\prime}\right| \omega_{1}, \tau^{\prime},\left|\eta^{\prime}, \tau^{\prime}\right| \omega_{2}^{\prime}\right) d \eta^{\prime} d \tau^{\prime}\right) d \omega .
$$


For fixed $\omega$ the function $\phi\left(x, \eta^{\prime}, \tau^{\prime}\right)$ is a non-degenerate multiphase function parametrizing the Lagrangian pair $\left(\Lambda_{0}, \Lambda_{1}\right)$. Therefore, we conclude that $I(a, \phi) \in I^{m+e / 2, p+e / 2}$.

Now considering arbitrary excesses, we shall show that $I(a, \phi) \in I^{m+e_{0} / 2, p+e_{1} / 2}\left(\Lambda_{0}, \Lambda_{1}\right)$. Indeed, by Proposition $\llbracket .36$, we can rewrite $\phi(x, \eta, \tau)=\psi\left(x, \eta^{\prime}, \tau^{\prime}\right)+\eta^{\prime \prime} \tau^{\prime \prime} /\left|\eta^{\prime}, \tau^{\prime}\right|$, where $\psi\left(x, \eta^{\prime}, \tau^{\prime}\right)$ is a multiphase function with excesses $\left(e_{0}, e_{0}+e_{1}-e, e_{0}\right)$ which parametrizes the Lagrangian pair $\left(\Lambda_{0}, \Lambda_{1}\right)$ near the point $\left(x_{0}, \xi_{0}\right)$. Then we have

$$
I(\phi, a)=\int e^{i \psi\left(x, \eta^{\prime}, \tau^{\prime}\right)} b\left(x, \eta^{\prime}, \tau^{\prime}\right) d \eta^{\prime} d \tau^{\prime},
$$

where $b\left(x, \eta^{\prime}, \tau^{\prime}\right)=\int e^{i \eta^{\prime \prime} \tau^{\prime \prime} /\left|\eta^{\prime}, \tau^{\prime}\right|} a\left(x, \eta^{\prime}, \eta^{\prime \prime}, \tau^{\prime}, \tau^{\prime \prime}\right) d \eta^{\prime \prime} d \tau$.

Since $a \in S^{m^{\prime}, p^{\prime}}$ and supp $a$ is contained in some conic neighbourhood of $\left(x_{0}, \eta_{0}, 0\right)$, we get $b \in S^{m^{\prime}+\left(e-e_{0}\right), p^{\prime}+\left(e-e_{0}\right)}$ by Proposition 1L.47. This means that $I(a, \phi)=I(b, \psi)$ where $b \in S^{m^{\prime}+\left(e-e_{0}\right), p+\left(e-e_{0}\right)}$ and $\psi$ is a multiphase function with excesses $\left(e_{0}, e_{0}+e_{1}-e, e_{0}\right)$ near $\left(x_{0}, \eta_{0}^{\prime}, 0\right)$ in $X \times\left(\mathbb{R}^{k-\left(e-e_{0}\right)} \times \mathbb{R}^{l-\left(e-e_{0}\right)} \backslash 0\right)$. The first step of the proof asserts that $I(b, \psi) \in I^{m^{\prime \prime}, p^{\prime \prime}}\left(\Lambda_{0}, \Lambda_{1}\right)$, where the orders $m^{\prime \prime}, p^{\prime \prime}$ satisfy

$$
\begin{array}{ll}
m^{\prime \prime}=m^{\prime}+\left(e-e_{0}\right)+\left(k+l-2\left(e-e_{0}\right)-e_{0}\right) / 2+n / 4 & =m+e_{0} / 2 \\
p^{\prime \prime}=p^{\prime}+\left(e-e_{0}\right)+\left(k-\left(e-e_{0}\right)-\left(e_{0}+e_{1}-e\right)\right) / 2+n / 4 & =p+e_{1} / 2
\end{array}
$$

Hence, $I(a, \phi) \in I^{m+e_{0} / 2, p+e_{1} / 2}\left(\Lambda_{0}, \Lambda_{1}\right)$.

As a corollary of this theorem, Theorem $\mathbb{L . 2 8}$ and Theorem 1.58 , we obtain

Corollary 1.63. Let $X, Y$ be smooth manifolds and $\Gamma$ be a canonical relation from $T^{*} Y \backslash 0$ to $T^{*} X \backslash 0$ and $\left(\Lambda_{0}, \Lambda_{1}\right)$ be a cleanly intersecting pair of Lagrangian submanifold in $T^{*} Y \backslash 0$. Assume that $\Gamma \times \Lambda_{0}, \Gamma \times \Lambda_{1}$ and $T^{*} X \times \Delta_{T^{*} Y}$ form an intersecting triple. If $u \in I^{m, p}\left(\Lambda_{0}, \Lambda_{1}\right), k_{Q} \in I^{q}\left(X \times Y, \Gamma^{\prime}\right)$, then $Q u \in I^{m+e_{0} / 2, p+e_{1} / 2}\left(\Gamma \circ \Lambda_{0}, \Gamma \circ \Lambda_{1}\right)$, where $e_{0}, e_{1}, e$ are the excesses of the intersecting triple above. 


\section{Compositions of paired Lagrangian distributions}

Composition theorems for Fourier integral operators have been understood in a great variety of cases. For instance, if two canonical relations satisfy a certain geometric condition, then the composition of two corresponding Fourier integral operators is again a Fourier integral operator. Moreover, the symbolic calculus of the composition was asserted, see [Hör85, Theorem 25.2.3].

The analogous question for the composition of Fourier integral operators and paired Lagrangian distributions has not been studied systematically. Joshi proposed and proved a part of composition theorem under a more general condition. However, the symbolic formulation of this statement, the order and principal symbol of the composite were not given, see [ ]0s99] for details.

In the same situation, the composition theorem for paired Lagrangian operators has been proved only in a particular case. More precisely, only the case of paired Lagrangian distributions when the first canonical relation is the diagonal and the second one is a flow-out manifold, the one obtained from the flow of the characteristic set under a bicharactertistic flow. For details, see [AU85, Theorem 0.1] and [Jos98, Theorem 11.2]. More general cases of canonical relations are unknown.

This chapter is devoted to composition theorems for classes of paired Lagrangian distributions, in general case under some geometric condition on their canonical relations. Our first main result is the composition of Fourier integral operators and paired Lagrangian distributions, under a certain condition on the canonical relations, see Theorem 2.8. Note that when this condition fails, the composite, in general, is not a PLD, see Theorem 2.46 and Corollary 2.49 below. As a consequence of Theorem 2.8, we prove a composition theorem for FIOs which generalizes the cleanly composition [Hör85, Theorem 25.2.3] to the weak canonical relations, see Proposition 2.10 . 


\subsection{Composition of Fourier integral operators and paired Lagrangian distributions}

Throughout this section we make the following assumptions: $X, Y, Z$ are subset in Euclidean spaces; $C$ is a canonical relation from $T^{*} Y \backslash 0$ to $T^{*} X \backslash 0$ and $\left(C_{0}, C_{1}\right)$ is an intersecting paired of canonical relations from $T^{*} Z \backslash 0$ to $T^{*} Y \backslash 0$.

In chapter 1, the intersecting triple condition is introduced to preserve the cleanness of Lagrangian pair under composition of relations. Now we give more details on this condition. Moreover, we will show that this condition is also sufficient for strong composition of FIOs and PLDs.

\subsubsection{Geometric condition}

Proposition 2.1 (Joshi). If the submanifolds $X, Y, Z$ form an intersecting triple, then near any point there exist local coordinates such that each submanifold is given by a subset of coordinates vanishing.

As a corollary, one obtains

Corollary 2.2. If submanifolds $X, Y, Z$ of $M$ is an intersecting triple then for any smooth function $f$ on $X$ which vanishes on $X \cap(Y \cup Z)$, there exists an extension $h$ of $f$ on $M$ such that $h$ vanishes on $Y \cup Z$.

Now we prove a criterion for locally clean intersections.

Lemma 2.3. Let $Y, Z$ be submanifolds of a smooth manifold $X$ and $\Sigma$ be a closed submanifold of $Y$ and $Z$ such that for all $p \in \Sigma$, one has

$$
T_{p} \Sigma=T_{p} Y \cap T_{p} Z
$$

Then $Y$ and $Z$ intersect cleanly locally near $\Sigma$, that is, there exists a neighbourhood $U$ of $\Sigma$ in $X$ satisfying

$$
Y \cap Z \cap U=\Sigma \text {. }
$$

Proof. Because of the following inclusions $\Sigma \subseteq Y \subseteq X$, there exist coordinates $x=$ $\left(x^{\prime}, x^{\prime \prime}, x^{\prime \prime \prime}\right)$ such that

$$
Y=\left\{x^{\prime}=0\right\}, \Sigma=\left\{x^{\prime}=0, x^{\prime \prime}=0\right\}, x=\left(x_{1}, \ldots, x_{k}, x_{k+1}, \ldots, x_{k+l}, x_{k+l+1}, \ldots, x_{d}\right) .
$$


Then the tangent space at $p=\left(0,0, x^{\prime \prime \prime}\right) \in \Sigma$ is determined by equations

$$
\left(t^{\prime}, t^{\prime \prime}, t^{\prime \prime \prime}\right) \in \mathbb{R}^{d}, t^{\prime}=0, t^{\prime \prime}=0 .
$$

Since $Z$ is a submanifold of $X$, there exists a smooth function $F=\left(F_{1}, \ldots, F_{m}\right)$ such that locally near $p$,

$$
Z=\{x \mid F(x)=0\},
$$

and $d F$ are linearly independent at $Z$. A vector $t \in T_{p} Y \cap T_{p} Z$ if and only if $t^{\prime}=0$ and $d F(p) t=0$. By relation (2.1), one obtains

$$
\frac{\partial F}{\partial x^{\prime \prime}}\left(0,0, x^{\prime \prime \prime}\right) t^{\prime \prime}+\frac{\partial F}{\partial x^{\prime \prime \prime}}\left(0,0, x^{\prime \prime \prime}\right) t^{\prime \prime \prime}=0 \Longleftrightarrow t^{\prime \prime}=0 .
$$

Hence

$$
\frac{\partial F}{\partial x^{\prime \prime \prime}}\left(0,0, x^{\prime \prime \prime}\right) \equiv 0, \text { rank } \frac{\partial F}{\partial x^{\prime \prime}}\left(0,0, x^{\prime \prime \prime}\right)=l .
$$

Since $\Sigma \subseteq Z$, one obtains $F\left(0,0, x^{\prime \prime \prime}\right)=0$. Then, by standard calculus, one has

$$
F(x)=A(x) x^{\prime}+B(x) x^{\prime \prime} \Longrightarrow d F(x)=A(x) d x^{\prime}+B(x) d x^{\prime \prime},
$$

where $A \in \mathbb{M}^{m \times k}, B \in \mathbb{M}^{m \times l}$ are matrices of smooth functions. Consequently,

$$
\frac{\partial F}{\partial x^{\prime \prime}}\left(0,0, x^{\prime \prime \prime}\right)=B\left(0,0, x^{\prime \prime \prime}\right) \text {. }
$$

Therefore, $B\left(0,0, x^{\prime \prime \prime}\right)$ has full rank $l$. Without loss of generality, assume that $B(x)$ has rank $l$ in a neighbourhood $U_{p}$. Hence, in $U_{p}$ the set $Y \cap Z$ is determined by the following system:

$$
\begin{aligned}
F(x)=0, x^{\prime}=0, x \in U_{p} & \Longleftrightarrow A(x) x^{\prime}=0, B(x) x^{\prime \prime}=0, x^{\prime}=0, x \in U_{p} \\
& \Longleftrightarrow x^{\prime}=0, x^{\prime \prime}=0, x \in U_{p},
\end{aligned}
$$

as $B$ has rank $l$. In other words, $Y \cap Z \cap U_{p}=\Sigma \cap U_{p}$. The proof is completed by setting $U=\bigcup_{p \in \Sigma} U_{p}$.

Remark 2.4. Away from $\Sigma$, the intersection $Y \cap Z$ freely has any type of singularities. In other words, $Y, Z$ may not intersect cleanly far from $\Sigma$. The analogous statement does not hold if one replaces (2.1) by a weaker condition: The following space

$$
\left(\frac{T_{p} Y \cap T_{p} Z}{T_{p} \Sigma}\right), p \in \Sigma,
$$

has a positive constant dimension.

If $\Sigma=Y \cap Z$, then trivially one has globally clean intersection. 


\subsubsection{Weak compositions}

We now reformulate the following result whose details can be found in [Jos99]. Note that for our aim, we translate this into the language of canonical relations and operators without the Sobolev stable orders.

Theorem 2.5 (Joshi). Let $C_{0}, C_{1}$ be a cleanly intersecting pair of canonical relations from $T^{*} Y \backslash 0$ to $T^{*} X \backslash 0$ and $C$ be a canonical relation from $T^{*} Z \backslash 0$ to $T^{*} Y \backslash 0$ such that

$$
\left(T^{*} X \backslash 0\right) \times \Delta_{T^{*} Y \backslash 0} \times\left(T^{*} Z \backslash 0\right), C_{0} \times C, C_{1} \times C
$$

form an intersecting triple. Then the weak composition of properly supported operators holds:

$$
I\left(C_{0}, C_{1}\right) \circ I(C) \subseteq I\left(C_{0} \circ C, C_{1} \circ C\right) .
$$

Sketch of Proof. By using partition of Unity, for any conic neighbourhood $W$ of $C_{0} \cap C_{1}$, one has the following finite decomposition

$$
A=A_{0}+\sum A_{j}, j \in J
$$

where $\mathrm{WF}^{\prime}\left(A_{0}\right) \subset W$ and $A_{j} \in I\left(C_{0} \backslash W\right)+I\left(C_{1} \backslash W\right)$. Using composition theorem for FIOs, it is sufficient to consider the composition of $A_{0}$ and $B$.

Hence, we suppose that $\mathrm{WF}^{\prime}(A)$ is contained in a small conic neighbourhood of $C_{0} \cap C_{1}$ and $\mathrm{WF}^{\prime}(B)$ is contained in a sufficiently small conic neighbourhood. This leads to an important effect on composition that $C_{0} \circ C, C_{1} \circ C,\left(C_{0} \cap C_{1}\right) \circ C$ are proper compositions. Note that the compositions of canonical relations hold locally with respect to the wave front relations of initial operators, but the weak composition law is true globally.

The geometric part asserts that $C_{0} \circ C, C_{1} \circ C$ form a cleanly intersecting pair of canonical relations while the analysis part shows that the composed operator is a paired Lagrangian distribution.

The geometric part goes as follows: By Theorem $[\mathrm{L}$, the following properties locally hold:

- $C_{0} \circ C, C_{1} \circ C$ are canonical relations,

- $\Sigma=\left(C_{0} \cap C_{1}\right) \circ C$ is an isotropic relation, submanifold of $C_{0} \circ C, j=0,1$,

- For each $p \in \Sigma$, one has

$$
T_{p} \Sigma=T_{p}\left(C_{0} \circ C\right) \cap T_{p}\left(C_{1} \circ C\right) .
$$


Hence, $C_{0} \circ C$ and $C_{1} \circ C$ intersect cleanly near $\left(C_{0} \cap C_{1}\right) \circ C$ by Lemma 2.3 .

We prove the second part by using geometric characterization of paired Lagrangian distributions. Without loss of generality, we assume that $C_{0} \circ C, C_{1} \circ C$ intersect cleanly.

Firstly, decompose composition into fundamental operations: For all $A \in I\left(C_{0}, C_{1}\right), B \in$ $I(C)$, one has

$$
A \circ B=\pi_{*} \Delta^{*}\left(k_{A} \otimes k_{B}\right),
$$

where $\Delta$ is the diagonal map

$$
X \times Y \times Z \ni(x, y, z) \mapsto(x, y, y, z) \in X \times Y \times Y \times Z,
$$

and $\pi$ is the projection from $X \times Y \times Z$ into $X \times Z$.

Secondly, remove the singular part of the tensor product $k_{A} \otimes k_{B}$. Although

$$
k_{A} \otimes k_{B} \notin I\left(C_{0} \times C, C_{1} \times C\right),
$$

one still has a small perturbation

$$
k_{A} \otimes k_{B}=\alpha+\beta, \quad \alpha \in I\left(C_{0} \times C, C_{1} \times C\right), \quad \pi_{*} \Delta^{*}(\beta) \in C^{\infty} .
$$

Therefore, it is sufficient to prove that

$$
\pi_{*} \Delta^{*}\left(I\left(C_{0} \times C, C_{1} \times C\right)\right) \subseteq I\left(C_{0} \circ C, C_{1} \circ C\right) .
$$

Finally, relation (2.10) is a consequence of the push-forward Theorem and the following property: every smooth homogeneous of degree zero function on $D:=T^{*} X \times \Delta_{T^{*} Y} \times$ $T^{*} Z$ which vanishes on $\left(C_{0} \times C\right) \cap D,\left(C_{1} \times C\right) \cap D$ can be extended to a smooth homogeneous of degree zero function on $T^{*}(X \times Y \times Y \times Z)$ which vanishes on $C_{0} \times C, C_{1} \times C$. The proof is completed by applying Corollary 2.2 .

Remark 2.6. Although $C_{0} \circ C, C_{1} \circ C$ may have bad intersections globally, the isotropic subset $\Sigma$ is an intrinsic part which contains singularities of the composed operator. In general, $C_{0} \circ C, C_{1} \circ C$ does not form a cleanly intersecting paired, even locally in $\Sigma$.

Self-intersections of canonical relations are avoidable if we consider only proper, connected compositions. Then $\Sigma$ is a smooth subset of $C_{0} \circ C \cap C_{1} \circ C$ which is separated from the remainder. All singularities of composed operators come from $\Sigma$, so we can neglect all other parts of the intersection $C_{0} \circ C \cap C_{1} \circ C$.

In order to get global cleanness of the pair $C_{0} \circ C, C_{1} \circ C$, a further assumption should be added. For instance, $C_{0} \circ C \cap C_{1} \circ C=\left(C_{0} \cap C_{1}\right) \circ C$ is a sufficient condition. 
Remark 2.7. The same procedure was used to prove the weak composition of FIOs: If $D$ and $C_{0} \times C$ intersect cleanly then microlocally, one get

$$
I\left(C_{0}\right) \circ I(C) \subseteq I\left(C_{0} \circ C\right)
$$

However, similar statement fails if at least one canonical relation contains zero section. See Proposition 2.10 for further discussion.

\subsubsection{Strong compositions}

By using oscillatory integral representation as in Chapter $\mathbb{W}$, we shall improve the composition of FIOs and PLDs. Being consistent with the action of Fourier integral operators on paired Lagrangian distributions, we state a slightly different form from the weak composition theorem.

Theorem 2.8. Let $C$ be a canonical relation from $T^{*} Y \backslash 0$ to $T^{*} X \backslash 0$ and $\left(C_{0}, C_{1}\right)$ be two canonical relations from $T^{*} Z \backslash 0$ to $T^{*} Y \backslash 0$ such that $C \times C_{0}, C \times C_{1}$ and $T^{*} X \times \Delta_{T^{*} Y} \times T^{*} Z$ form an intersecting triple. If $A \in I^{m}(X, Y ; C)$ and $B \in I^{p_{0}, p_{1}}\left(Y, Z ; C_{0}, C_{1}\right)$ are two properly supported operators, then the composite

$$
A \circ B \in I^{m+p_{0}+e_{0} / 2, m+p_{1}+e_{1} / 2}\left(X, Z ; C \circ C_{0}, C \circ C_{1}\right)
$$

where $e_{j}$ is the excess of the composition $C \circ C_{j}, j=0,1$.

Proof. We use the method of the proof of the compositions of Fourier integral operators. Let $\phi(x, y, \theta)$ be a regular phase function parametrizing the canonical relation $C$ and $\psi(y, z, \eta, \tau)$ be a regular multiphase function parametrizing the pairs $\left(C_{0}, C_{1}\right)$. One only needs to prove this conclusion locally. Assume that

$$
\begin{aligned}
& k_{A}(x, y)=\int e^{i \phi(x, y, \theta)} a(x, y, \theta) d \theta, \\
& k_{B}(y, z)=\int e^{i \psi(y, z, \eta, \tau)} b(y, z, \eta, \tau) d \eta d \tau,
\end{aligned}
$$

where $a \in S^{m^{\prime}}(X \times Y), b \in S^{p_{0}^{\prime}, p_{1}^{\prime}}(Y \times Z)$. Then we have

$$
k_{A \circ B}(x, z)=\int e^{i \phi(x, y, \theta)+i \psi(y, \eta, \tau)} a(x, y, \theta) b(y, \eta, \tau) d \theta d \eta d \tau .
$$

Step 1. Lifting the multiphase phase function. Write

$$
\Psi(x, z, \omega, \theta, \eta, \tau)=\phi(x, \omega /|\eta, \eta, \tau|, \theta)+\psi(\omega /|\eta, \eta, \tau|, \eta, \tau) .
$$


By Theorem $\mathbb{L 2 8}$, the function $\Psi$ is a multiphase function parametrizing the pair $C \circ$ $C_{0}, C \circ C_{1}$ with excesses $e_{0}, e_{1}, e$.

Step 2. Lifting the amplitude function. Using similar argument in the proof of Theorem 15.58 , there exists a constant $c>0$ such that whenever $\phi_{y}(x, y, \theta)+\psi_{y}(y, z, \eta, \tau)=0$, we have $|\theta| / c \leq|\eta, \tau| \leq c|\theta|$. Thus, only the part satisfying $|\theta| \simeq|\eta, \tau|$ contributes to the singularities of $k_{A \circ B}$. Hence, the amplitude function $a(x, y, \theta) b(y, z, \eta, \tau)$ belongs to the symbol classes $S^{m_{1}, m_{2}}(X \times Z)$.

Step 3. Using Theorem $1 \mathrm{L.62}$ which provides oscillatory integral representation of paired Lagrangian distributions via multiphase functions, one obtains the conclusion of the theorem.

Remark 2.9. If $C$ is the graph of a canonical transformation, then the assumption in the theorem automatically holds. Compositions of paired Lagrangian operators and Fourier integral operators are formulated and proved in the same way.

Proposition 2.10. Let $C$ be a canonical relation from $T^{*} Y \backslash 0$ to $T^{*} X \backslash 0$ and $C_{1}$ be a weak canonical relation from $T^{*} Z \backslash 0$ to $T^{*} Y$ such that

$$
\left(T^{*} X \backslash 0\right) \times \Delta_{T^{*} Y} \times\left(T^{*} Z \backslash 0\right), C \times C_{1}, 0_{X \times Y} \times C_{1}
$$

is an intersecting triple. Then for all properly supported operators $A \in I^{m}(C), B \in$ $I^{m_{1}}\left(C_{1}\right)$, one has

$$
A \circ B \in I^{m+m_{1}+e / 2, m_{1}+e_{0} / 2-\left(d_{x}+d_{y}\right) / 4}\left(C \circ C_{1}, 0_{X \times Y} \circ C_{1}\right),
$$

where e, $e_{0}$ are excesses of composition $C \circ C_{1}, 0_{X \times Y} \circ C_{1}$, respectively.

Idea of the first Proof. The geometric part, which asserts that $C \circ C_{1}, 0_{X \times Y} \circ C_{1}$ intersect cleanly, follows from the intersecting triple. The second part works well by using the method as in the proof of Theorem 2.8, except that the second step needs a slight modification.

Second Proof. Rewrite composition in terms of fundamental operations as in (2.8)). The proof is divided into three steps:

Step 1. Describe the singularities of the tensor product. We use the following fact: If $u \in I^{p_{1}}\left(X ; \Lambda_{1}\right)$ and $v \in I^{p_{2}}\left(Y ; \Lambda_{2}\right)$, then

$$
u \otimes v \in I^{p_{1}+p_{2}, p_{2}-d_{1} / 4, p_{1}-d_{2} / 4}\left(X \times Y ; \Lambda_{1} \times \Lambda_{2}, 0_{X} \times \Lambda_{2}, \Lambda_{1} \times 0_{Y}\right),
$$


where $d_{1}=\operatorname{dim} X, d_{2}=\operatorname{dim} Y$ and the right hand-side is the space of distributions associated with three Lagrangian manifolds with the following properties: $\Lambda_{1} \times 0_{Y} \cap 0_{X} \times \Lambda_{2}=\emptyset$. This implies that this class, indeed, consists of sum of paired Lagrangian distributions. Using the fact above for $k_{A} \in I^{m}\left(X \times Y ; C^{\prime}\right), k_{B} \in I^{m_{1}}\left(Y \times Z ; C_{1}^{\prime}\right)$, we obtain

$$
k_{A} \otimes k_{B} \in I^{m+m_{1}, m_{1}-\left(d_{x}+d_{y}\right) / 4, m-\left(d_{y}+d_{z}\right) / 4}\left(X \times Y \times Y \times Z ; L_{1}, L_{2}, L_{3}\right),
$$

where $L_{1}=C \times C_{1}, L_{2}=0_{X \times Z} \times C_{1}, L_{3}=C \times 0_{Y \times Z}$.

Step 2. Eliminate the singular part of the tensor product. Note that $P=\pi_{*} \Delta^{*}$ is a FIO of order zero associated with the canonical relation $\Gamma$ from $T^{*}(X \times Y \times Y \times Z) \backslash 0$ to $T^{*}(X \times Z)$ defined by

$$
\Gamma^{\prime}=N_{M}^{*}\left(\Delta_{X \times Y \times Z}\right), M=(X \times Y \times Z) \times(X \times Y \times Z) .
$$

Since $\Gamma \circ L_{3}=\emptyset$, only the part microlocally near $L_{1} \cup L_{2}$ contributes to singularities of $k_{A \circ B}$. Moreover, $\Gamma \circ L_{1}=C \circ C_{1}, \Gamma \circ L_{2}=0_{X \times Z} \circ C_{1}$ and away from $L_{3}$, one has:

$$
I^{\mu_{1}, \mu_{2}, \mu_{3}}\left(L_{1}, L_{2}, L_{3}\right) \subset I^{\mu_{1}, \mu_{2}}\left(L_{1} \backslash L_{3}, L_{2} \backslash L_{3}\right) .
$$

Hence, the conclusion follows if one shows that

$$
P: I^{\mu_{1}, \mu_{2}}\left(L_{1}, L_{2}\right) \subseteq I^{\mu_{1}+e_{1} / 2, \mu_{2}+e_{2} / 2}\left(\Gamma \circ L_{1}, \Gamma \circ L_{2}\right)
$$

Step 3. Compose of the regular part. By applying Theorem 2.8 , the proof is completed.

Example 2.11. Let $X$ be a $k$-dimensional submanifold of $Z$. Consider the following canonical relations:

$$
\begin{array}{rlrl}
C & =\Delta_{T^{*} X} & & \subset\left(T^{*} X \backslash 0\right) \times\left(T^{*} X \backslash 0\right), \\
C_{1} & =N_{X \times Z}^{*}\left(\Delta_{X}\right)^{\prime} & & \subset T^{*} X \times\left(T^{*} Z \backslash 0\right), \\
C_{0} & =N_{X \times Z}^{*}(X \times X)^{\prime} & \subset T^{*} X \times\left(T^{*} Z \backslash 0\right) .
\end{array}
$$

Then the assumption of Proposition 2.10 is fulfilled and one obtains

$$
I^{m}\left(\Delta_{X}\right) \circ I^{p}\left(C_{1}\right) \subseteq I^{m+p, p-k / 2}\left(C_{1}, C_{0}\right) .
$$

The condition in Theorem 2.8 is natural to obtain paired Lagrangian distributions. When this condition is not fulfilled, in general, even $\left(C \circ C_{0}, C \circ C_{1}\right)$ is a cleanly intersecting pair of canonical relations, the composed operator is not a paired Lagrangian distribution associated with this pair. For more precise statement, see Theorem 2.46 . 


\subsection{Composition of paired Lagrangian distributions near their intersections}

Let $\left(C_{0}, C_{1}\right)$ and $\left(\widetilde{C}_{0}, \widetilde{C}_{1}\right)$ be pairs of canonical relations from $T^{*} Y \backslash 0$ to $T^{*} X \backslash 0$ and from $T^{*} Z \backslash 0$ to $T^{*} Y \backslash 0$, respectively. We shall investigate compositions $A \circ B$ of properly supported operators $A \in I\left(C_{0}, C_{1}\right)$ and $B \in I\left(\widetilde{C}_{0}, \widetilde{C}_{1}\right)$. By abstract composition, $\mathrm{WF}^{\prime}(A \circ B)$ is contained in $\bigcup_{i, j=0,1} C_{j} \circ \widetilde{C}_{k}$. According to geometric property of the system

$$
L=\left\{C_{j} \circ \widetilde{C}_{k}, j, k=0,1\right\},
$$

the composite can be in various forms. In general, $L$ can be a system of canonical relations which might have non-clean intersections. Moreover, the compositions of isotropic canonical relations $C_{i} \cap C_{j}$ and $\widetilde{C}_{k} \cap \widetilde{C}_{l}$ for $i, j, k, l=0,1$ have a strong effect on the resulting operators. Note that away from the intersection of two Lagrangian manifolds, the corresponding PLDs are indeed Lagrangian distributions. So if $\left(C_{0} \cap C_{1}\right) \circ\left(\widetilde{C}_{0} \cap \widetilde{C}_{1}\right)=\emptyset$, then the composition of related paired Lagrangian distributions can be written as sum of compositions of FIOs and PLDs by using microlocal decomposition and the fact above. These typical results have been already investigated in the last section. Therefore, in this section we consider only the non-trivial cases

$$
\left(C_{0} \bigcap C_{1}\right) \circ\left(\widetilde{C}_{0} \bigcap \widetilde{C}_{1}\right) \neq \emptyset
$$

We introduce a geometric condition which ensures that all involved compositions of isotropic canonical relations are smooth isotropic relations. Furthermore, we prove that under this condition, all resulting relations obey a nice law and the composition operators belong to the class of distributions associated with this system.

Throughout this section we make the following fundamental assumption:

Assumption 1. The set $\left\{C_{j} \circ \tilde{C}_{k}, j, k=0,1\right\}$ forms a clean system of $\kappa$ Lagrangian manifolds, i.e., the intersection of any number of those manifolds is again a submanifold whose tangent plane at each point is equal to the intersection of those tangent planes.

\subsubsection{Geometric condition}

Definition 2.12. A family $L=\left\{L_{j}, j \in J\right\}$, where $J$ is a finite set, is called a cleanly intersecting system (CIS) of submanifolds if locally each possible intersection is given by a subset of vanishing local coordinates. 
Remark 2.13. For $|J|=2$, a cleanly intersecting system is a cleanly intersecting pair. Similarly, a system of three submanifolds forms a cleanly intersecting system if and only if it is a cleanly intersecting triple.

As a direct consequence of Definition 2.12, a CIS has the following properties:

Proposition 2.14. Let $L=\left\{L_{j}, j \in J\right\}$ be a cleanly intersecting system of smooth submanifolds in X. Then one has:

1. For all $A \subseteq J$, the subsystem $\left\{L_{j}, j \in A\right\}$ is a CIS.

2. For all $A \subseteq J, L_{A}:=\bigcap_{j \in A} L_{j}$ is a smooth submanifold and, for all $p \in L_{A}$, one has

$$
T_{p} L_{A}=\bigcap_{j \in A} T_{p} L_{j}
$$

3. For all $A \subseteq J$ and any point $p \in L_{A}$ one has

$$
\operatorname{dim}\left(\sum_{j \in A} T_{p} L_{j}\right)=\sum_{B \subseteq A}(-1)^{|B|+1} \operatorname{dim} L_{B} .
$$

4. For all $p \in \cup L_{j}, J_{p}=\left\{j \in J \mid p \in L_{j}\right\}$. In some neighbourhood of $p$ in $X$, there exist coordinates $x_{1}, \ldots, x_{n}$ and subsets of $B_{j} \subseteq\{1, \ldots, n\}$ for $j \in J_{p}$ such that $L_{j}=\left\{x_{k}=0, k \in B_{j}\right\}$.

Example 2.15. Let $\left(C_{0}, C_{1}\right)$ and $\left(\widetilde{C}_{0}, \widetilde{C}_{1}\right)$ be cleanly intersecting pairs of smooth submanifolds in $X$ and in $Y$, respectively. Then $\left\{C_{j} \times \widetilde{C}_{k} \mid j, k=0,1\right\}$ is a CIS in $X \times Y$.

Proof. Let define

$$
L_{2 j+k}=C_{j} \times \widetilde{C}_{k}, j, k=0,1 .
$$

Since $\left(C_{0}, C_{1}\right)$ is a cleanly intersecting system in $X$, there exist coordinates $x_{1}, \ldots, x_{n}$ in $X$ such that $C_{0}=\left\{x^{\prime}=0, x^{\prime \prime}=0\right\}$ and $C_{1}=\left\{x^{\prime}=0, x^{\prime \prime \prime}=0,\right\}$. Similarly, there exist coordinates $y_{1}, \ldots, y_{m}$ in $Y$ such that $\widetilde{C}_{0}=\left\{y^{\prime}=0, y^{\prime \prime}=0\right\}$ and $\widetilde{C}_{1}=\left\{y^{\prime}=0, y^{\prime \prime \prime}=0\right\}$. Then in the coordinates $x, y$ of $X \times Y, C_{j} \times \widetilde{C}_{k}$ is given by some coordinates vanishing. Hence, the system $\left\{L_{j}, j=0,1,2,3\right\}$ forms a CIS. Furthermore, this system satisfies:

$$
\begin{aligned}
L_{2 j+0} \cap L_{2 j+1} & =C_{j} \times\left(\widetilde{C}_{0} \cap \widetilde{C}_{1}\right) \\
L_{k} \cap L_{2+k} & =\left(C_{1} \cap C_{2}\right) \times \widetilde{C}_{k}, \\
L_{0} \cap L_{3}=L_{1} \cap L_{2} & =\left(C_{0} \cap C_{1}\right) \times\left(\widetilde{C}_{0} \cap \widetilde{C}_{1}\right), \\
\bigcap_{j \neq k} L_{j} & =\left(C_{0} \cap C_{1}\right) \times\left(\widetilde{C}_{0} \cap \widetilde{C}_{1}\right), \quad \forall j, k \in\{0,1,2,3\} \\
L_{0} \cap L_{1} \cap L_{2} \cap L_{3} & =\left(C_{1} \cap C_{2}\right) \times\left(\widetilde{C}_{0} \cap \widetilde{C}_{1}\right) .
\end{aligned}
$$


Definition 2.16. Let $D$ be a submanifold of manifold $X$ and $L$ be a subset of $X$. We say that $D$ has the LIFT property (with respect to $L$ ) if, for every smooth function $f$ on $D$ which vanishes on $D \cap L$, there exists a smooth function $\tilde{f}$ on $X$ such that $\left.\tilde{f}\right|_{D}=f$ and $\left.\tilde{f}\right|_{L}=0$.

Example 2.17. If $L$ and $D$ are submanifolds of $X$ such that $D$ and $L$ intersect cleanly, then $(D, L)$ has the lift property. Similarly, if $\left\{D, L_{1}, L_{2}\right\}$ forms a cleanly intersecting triple, then $\left(D, L_{1} \cup L_{2}\right)$ has the lift property.

From now on we restrict to the case that $L$ is the union of a system of submanifolds because in composition theorems of paired Lagrangian distributions, one only meets the situation when $L$ is union of four closed, conic Lagrangian manifolds.

Let us recall some facts about conic manifolds. By a conic manifold $X$ we mean a smooth manifold equipped with a free, proper action of the group $\mathbb{R}_{+}$:

$$
\mathbb{R}_{+} \times X \rightarrow X, \quad(t, x) \mapsto t \cdot x
$$

This action defines an equivalent relation on $X$ as in the following sense: $x \sim y$ if and only if there exists a $t \in \mathbb{R}_{+}$, such that $y=t \cdot x$. An equivalent class is called a $\mathbb{R}_{+}$-orbit. By [DK00, Theorem 1.11.4], the space of $\mathbb{R}_{+}$-orbits $X / \mathbb{R}_{+}$is a smooth manifold and $X$ is a $\mathbb{R}_{+}$-principal fibre bundle over $X / \mathbb{R}_{+}$with the canonical projection

$$
\pi: X \rightarrow X / \mathbb{R}_{+} .
$$

In general, we obtain the following result:

Lemma 2.18. Let $X$ be a conic manifold and $\left\{L_{j}, j \in J\right\}$ be a CIS of conic submanifolds in $X$. Then their images $\left\{\pi\left(L_{j}\right), j \in J\right\}$ form a CIS in the space of $\mathbb{R}_{+}$-orbits $X / \mathbb{R}_{+}$.

Proof. Because $L_{j} \subseteq X$ is a conic submanifold, we have a free, proper action:

$$
\mathbb{R}_{+} \times L_{j} \rightarrow L_{j}
$$

Hence, $\pi\left(L_{j}\right)$ is a submanifold of $X / \mathbb{R}_{+}$and $\operatorname{codim} \pi\left(L_{j}\right)=\operatorname{codim} L_{j}$. So $\left\{\pi\left(L_{j}\right), j \in J\right\}$ is a system of submanifolds in $X / \mathbb{R}_{+}$. For any given point $\theta_{0} \in \bigcap_{j \in A} \pi\left(L_{j}\right), A \subseteq J$, we shall show that there exist local coordinates on $X / \mathbb{R}_{+}$near $\theta_{0}$ such that each $L_{j}, j \in A$ is 
given by some coordinates vanishing. Indeed, by [DK00, Theorem 1.11.4], there are a neighbourhood $S \subset X / \mathbb{R}_{+}$and a diffeomorphism

$$
\begin{array}{r}
\tau: \pi^{-1}(S) \rightarrow \mathbb{R}_{+} \times S \\
x \mapsto(r(x), \theta(x))
\end{array}
$$

such that, for all $t \in \mathbb{R}_{+}, x \in \pi^{-1}(S)$ :

$$
\pi(x)=\theta(x) \text { and } \tau(t \cdot x)=(\operatorname{tr}(x), \theta(x))
$$

Since $\theta_{0} \in \bigcap_{j \in A} \pi\left(L_{j}\right)$, the point $x\left(1, \theta_{0}\right) \in \bigcap_{j \in A} L_{j}$. By the assumption of CIS, there exist local coordinates $x$ such that

$$
L_{j} \cap \pi^{-1}(S)=\left\{x \in M \mid x_{k}=0, k \in \alpha_{j} \subseteq\{1,2 \ldots, n\}\right\}, j \in A .
$$

Therefore, as a submanifold in $X / \mathbb{R}_{+}$with coordinate $\theta$, we obtain

$$
\pi\left(L_{j}\right) \cap S=\left\{\theta \in S \mid x_{j}(1, \theta)=0, j \in \alpha_{j} \subseteq\{1,2 \ldots, n\}\right\}, \quad j \in A .
$$

Since $t \cdot x\left(1, \theta_{0}\right)=x\left(r, \theta_{0}\right) \in \bigcap_{j \in A} L_{j}$ by conic assumption of the submanifolds $L_{j}, j \in J$, we have $x_{k}\left(r, \theta_{0}\right)=0, k \in \alpha_{j}$. Differentiating with respect to $t$, we obtain $\partial_{r} x_{k}\left(1, \theta_{0}\right)=0$, $k \in \alpha_{j}$. Consequently,

$$
\left.\operatorname{rank}\left(\partial_{\theta} x_{k}, k \in \alpha_{j}\right)\right|_{\theta=\theta_{0}}=\left.\operatorname{rank}\left(d x_{k}, k \in \alpha_{j}\right)\right|_{r=1, \theta=\theta_{0}}=\left|\alpha_{j}\right| .
$$

Thus, $d_{\theta} x_{k}(1, \theta), k \in \alpha_{j}$ are linearly independent at $\theta_{0}$. Similarly, the system $\left\{d_{\theta} x_{k}(1, \theta), k \in\right.$ $\left.\bigcup_{j \in A} \alpha_{j}\right\}$ has rank $\left|\bigcup_{j \in A} \alpha_{j}\right|$ at $\theta_{0}$ and the linear independence of the differentials follows. This means that $\left\{\pi\left(L_{j}\right), j \in J\right\}$ forms a CIS in $X / \mathbb{R}_{+}$.

Proposition 2.19. Let $\left\{D, L_{j}\right\}_{j \in J}$ be a CIS in X. Then D satisfies the LIFT property with respect to $L=\bigcup_{j \in J} L_{j}$. More strictly, for any smooth function $f$ on $D$ which vanishes on $D \cap L$, there exists an extension $\tilde{f}$ on $X$ such that $\tilde{f}$ vanishes on the system L. Furthermore, if $X$ and $D, L$ are conic manifolds, then the LIFT property holds in the class of homogeneous of degree one functions.

Proof. By partition of unity, it suffices to prove this result locally. Suppose that $p \in$ $\bigcap_{j \in A} L_{j}, A \subseteq J$. By inductive on $|J|$, consider only the case $A=J$. We pick coordinates $x$ such that $L_{j}:=\left\{x_{k}=0, k \in \alpha_{j}\right\}$ and $D:=\left\{x_{k}=0, k \in \alpha_{0}\right\}$. For any $1 \leq k \leq n$, we 
define $J(k)=\left\{j \in J \mid k \in \alpha_{j}\right\}$. The germs of smooth functions which vanish on $\bigcup_{j \in J} L_{j}$ are generated by the following germs

$$
\begin{gathered}
x_{k} \mid 1 \leq k \leq n, J(k)=J, \\
x_{k_{1}} x_{k_{2}} \mid 1 \leq k_{1}, k_{2} \leq n, J\left(k_{1}\right) \bigcup J\left(k_{2}\right)=J, \quad J\left(k_{2}\right) \neq J, J\left(k_{1}\right) \neq J, \\
x_{k_{1}} x_{k_{2}} x_{k_{3}} \mid 1 \leq k_{1}, k_{2}, k_{3} \leq n, \bigcup_{i=1}^{3} J\left(k_{i}\right)=J, \quad \bigcup_{i \neq j} J\left(k_{i}\right) \neq J, j=1,2,3, \\
\vdots \\
\prod_{i=1}^{|J|} x_{k_{i}}\left|1 \leq k_{i} \leq n, \bigcup_{i=1}^{|J|} J\left(k_{i}\right)=J, \quad \bigcup_{i \neq j} J\left(k_{i}\right) \neq J, 1 \leq j \leq\right| J \mid .
\end{gathered}
$$

Similarly, on $D$ the local coordinates are $x_{j}, j \in\{1, \ldots, n\} \backslash \alpha_{0}$. Hence, near the point $p$ in manifold $D$, the submanifolds $D \cap L_{j}, j \in J$ are given by:

$$
D \cap L_{j}=\left\{x_{j}=0 \mid j \in \alpha_{j} \backslash \alpha_{0}\right\}
$$

Therefore, the germs of smooth functions near $p$ which vanish on $\bigcup\left(D \cap L_{j}\right)$ are generated by functions given as in (2.17)-(2.20) with $\alpha_{j}$ are replaced by $\alpha_{j} \backslash \alpha_{0}$. Obviously, these functions are restriction to $D$ of the functions given in (2.17)-(2.20) $)$. Hence, the first part holds.

To prove the second part, we apply the lift property for the corresponding CIS system in the space of $X / \mathbb{R}_{+}$. Indeed, assume that $f$ is a smooth function on $D$ homogeneous of degree 1 such that $f$ vanishes on $D \cap\left(\bigcup_{j \in J} L_{j}\right)$. Let $D^{c}=\pi(D), L_{j}^{c}=\pi\left(L_{j}\right), j \in J$, be the projections of $D, L_{j}, j \in J$ to $X / \mathbb{R}_{+}$, respectively. Then $f$ defines a unique smooth function $f^{c}$ on $D^{c}$ which vanishes on $D^{c} \cap L_{j}^{c}$. By Lemma $2.18,\left\{D^{c}, L_{j}^{c}, j \in J\right\}$ forms a CIS in $X / \mathbb{R}_{+}$. Applying the lift property for this system, there exists a smooth extension $\tilde{f}^{c}$ on $X / \mathbb{R}_{+}$such that $\tilde{f}^{c}$ vanishes on $D^{c} \cap\left(\bigcup L_{j}^{c}\right)$. Hence, $\tilde{f}^{c}$ can be extended to a smooth function $\tilde{f}$ on $X$ homogeneous of degree 1. Obviously, $\left.\tilde{f}\right|_{L_{j}}=0, j \in J$ and $\left.\tilde{f}\right|_{D}=f$ follow from $\left.\tilde{f}^{c}\right|_{D^{c} \cap L_{j}^{c}}=0$ and $\left.\tilde{f}^{c}\right|_{D^{c}}=f^{c}$.

Remark 2.20. Although a cleanly intersecting system $L$ of Lagrangian submanifolds is given by the vanishing of coordinates, in general, the oscillatory integral representation of distributions associated with $L$ is still quite complicated. For example, consider the following system of three Lagrangian submanifolds in $T^{*} \mathbb{R}^{n}$ :

$$
L_{1}=N^{*}\left\{x^{\prime \prime}=0, x_{1}=0\right\}, L_{2}=N^{*}\left\{x^{\prime \prime}=0, x_{2}=0\right\}, L_{3}=N^{*}\{x=0\},
$$


where $x=\left(x_{1}, x_{2}, x^{\prime \prime}\right)$. Then $\mathcal{M}$, the class of first order symbol vanishing on $L_{1}, L_{2}, L_{3}$, is generated by

$$
\left\{x^{\prime \prime}|\xi|, x_{1} x_{2}|\xi|, x_{1} \xi_{1}, x_{2} \xi_{2}\right\}
$$

Hence, $u \in \mathcal{E}^{\prime}\left(\mathbb{R}^{n}\right)$ belongs to $I\left(L_{1}, L_{2}, L_{3}\right)$ if and only if there exists $m \in \mathbb{R}$ such that for all multi-index $\alpha \in \mathbb{Z}^{n}, l \in \mathbb{Z}$

$$
\left|\left(|\xi| \partial_{\xi^{\prime \prime}}\right)^{\alpha^{\prime \prime}}\left(\xi_{2} \partial_{\xi_{2}}\right)^{\alpha_{2}}\left(\xi_{1} \partial_{\xi_{1}}\right)^{\alpha_{1}}\left(|\xi| \partial_{\xi_{1} \xi_{2}}^{2}\right)^{l} \hat{u}(\xi)\right| \leq C\langle\xi\rangle^{m},|\xi| \geq 1
$$

Lemma 2.21. Let $\left(C_{0}, C_{1}\right)$ and $\left(\widetilde{C}_{0}, \widetilde{C}_{1}\right)$ be cleanly intersecting pairs of canonical relations from $T^{*} Y \backslash 0$ to $T^{*} X \backslash 0$ and from $T^{*} Z \backslash 0$ to $T^{*} Y \backslash 0$, respectively. In addition, assume that $\left\{\left(T^{*} X \backslash 0\right) \times \Delta_{T^{*} Y \backslash 0} \times\left(T^{*} Z \backslash 0\right), C_{j} \times \widetilde{C}_{k}, j, k=0,1\right\}$ forms a CIS of five submanifolds. Then the following statements hold locally.

1. All compositions $C_{j} \circ \widetilde{C}_{k}, j, k=0,1$ are canonical relations from $T^{*} Z \backslash 0$ to $T^{*} X \backslash 0$.

2. All subsets $\left(C_{i} \cap C_{j}\right) \circ\left(\widetilde{C}_{k} \cap \widetilde{C}_{l}\right), i, j, k, l=0,1$ are conic isotropic submanifolds in $T^{*}(X \times Z) \backslash 0$.

3. The composition distributes over the intersection in the sense that for all $\gamma \in$ $\left(C_{i} \cap C_{j}\right) \circ\left(\widetilde{C}_{k} \cap \widetilde{C}_{l}\right)$,

$$
T_{\gamma}\left(\left(C_{i} \bigcap C_{j}\right) \circ\left(\widetilde{C}_{k} \bigcap \widetilde{C}_{l}\right)\right)=T_{\gamma}\left(C_{i} \circ \widetilde{C}_{k}\right) \bigcap T_{\gamma}\left(C_{j} \circ \widetilde{C}_{l}\right), i, j, k, l=0,1 .
$$

4. $\left\{C_{j} \circ \widetilde{C}_{k}, j, k=0,1\right\}$ is a pairwise cleanly intersecting system.

Proof. Note that the CIS assumption implies that $C_{j} \times \widetilde{C}_{k}$ and $\left(T^{*} X \backslash 0\right) \times \Delta_{T^{*} Y \backslash 0} \times\left(T^{*} Z \backslash 0\right)$ intersect cleanly. So the first part is a consequence of the clean composition of Lagrangian canonical relations.

The second part follows if one shows that all conditions in composition of isotropic relations are fulfilled. Let $\Sigma:=C_{0} \cap C_{1}, \widetilde{\Sigma}:=\widetilde{C}_{0} \cap \widetilde{C}_{1}$. The compositions of type $C_{j} \circ \widetilde{\Sigma}$ or $\Sigma \circ \widetilde{C}_{k}$ are well-defined. For example, the composition $C_{0} \circ \widetilde{\Sigma}$ is an isotropic relation since $\left\{D, C_{0} \times \widetilde{C}_{0}, C_{0} \times \widetilde{C}_{1}\right\}$ is an intersecting triple by CIS assumption.

We now show that $\Sigma \circ \widetilde{\Sigma}$ defines an isotropic relation. By Theorem 1 .4 or [BG8], Theorem 9.4], one needs to check that both $(\Sigma \times \widetilde{\Sigma}) \cap D$ and $T_{p}(\Sigma \times \widetilde{\Sigma}) \cap T_{p} D^{\sigma}, \quad \forall p \in$ $(\Sigma \times \widetilde{\Sigma}) \cap D$ have constant dimensions. Since $(\Sigma \times \widetilde{\Sigma}) \cap D=\bigcap_{i, j=0,1}\left(C_{j} \times \widetilde{C}_{k}\right) \cap D$, it has constant dimension by Proposition 2.14, Item 2. Besides, due to the symplectic complement, for all $p \in(\Sigma \times \widetilde{\Sigma}) \cap D$, one gains 


$$
\begin{aligned}
\operatorname{dim}\left(T_{p}(\Sigma \times \widetilde{\Sigma}) \cap T_{p} D^{\sigma}\right) & =2 \operatorname{dim} X+4 \operatorname{dim} Y+2 \operatorname{dim} Z-\operatorname{dim}\left(T_{p}(\Sigma \times \widetilde{\Sigma})^{\sigma}+T_{p} D\right) \\
& =2 \operatorname{dim} X+4 \operatorname{dim} Y+2 \operatorname{dim} Z-\operatorname{dim}\left(\left(\bigcap_{j, k} T_{p}\left(C_{j} \times \widetilde{C}_{k}\right)\right)^{\sigma}+T_{p} D\right) \\
& =2 \operatorname{dim} X+4 \operatorname{dim} Y+2 \operatorname{dim} Z-\operatorname{dim}\left(\sum_{i, k=0,1} T_{p}\left(C_{j} \times \widetilde{C}_{k}\right)+T_{p} D\right) .
\end{aligned}
$$

By Proposition 2.14, Item 3, the space $\sum_{i, k=0,1} T_{p}\left(C_{j} \times \widetilde{C}_{k}\right)+T_{p} D$ has constant dimension, so does $T_{p}(\Sigma \times \widetilde{\Sigma}) \cap T_{p} D^{\sigma}$.

The third part is proved similarly as in Theorem L.7. Let $\gamma \in \Sigma \circ \widetilde{\Sigma}$ and $p \in \Sigma \times \widetilde{\Sigma}$ such that $\pi(p)=\gamma$. Define

$$
\begin{aligned}
\Delta & =T_{p}\left(T^{*} X \times \Delta_{T^{*} Y} \times T^{*} Z\right), \\
L_{0} & =T_{p}\left(C_{0} \times \widetilde{C}_{1}\right), \quad L_{1}=T_{p}\left(C_{1} \times \widetilde{C}_{0}\right), \\
L_{2} & =T_{p}\left(C_{1} \times \widetilde{C}_{1}\right), \quad L_{3}=T_{p}\left(C_{1} \times \widetilde{C}_{1}\right) .
\end{aligned}
$$

Then one has

$$
\Delta \cap \sum_{j=0}^{3} L_{j}=\sum_{j=0}^{3}\left(\Delta \cap L_{j}\right)
$$

By taking the symplectic complement, we obtain

$$
\left(\bigcap_{j=0}^{3} L_{j}\right)+\Delta^{\sigma}=\bigcap_{j=0}^{3}\left(L_{j}+\Delta^{\sigma}\right) .
$$

On the other hand, for any vector subspace $A$, the following relation holds

$$
\left(A+\Delta^{\sigma}\right) \cap \Delta=(A \cap \Delta)+\Delta^{\sigma} .
$$

Indeed, since $\Delta^{\sigma} \subset \Delta$ one has $\left(A+\Delta^{\sigma}\right) \cap \Delta \supseteq(A \cap \Delta)+\left(\Delta^{\sigma} \cap \Delta\right)=(A \cap \Delta)+\Delta^{\sigma}$. To prove the inverse conclusion, take any element $a+d^{\sigma}=d \in\left(A+\Delta^{\sigma}\right) \cap \Delta$. Since $a=d-d^{\sigma} \in \Delta, d^{\sigma} \in \Delta^{\sigma}$, one gains $a+d^{\sigma} \in(A \cap D)+\Delta^{\sigma}$. Therefore,

$$
(A+\Delta)^{\sigma} \cap \Delta \subseteq(A \cap \Delta)+\Delta^{\sigma} .
$$

The relation (2.24) is verified.

Intersecting both side of ([2.23) with $\Delta$ and using (2.24), we conclude

$$
\frac{L_{0} \cap L_{1} \cap L_{2} \cap L_{3} \cap \Delta}{\Delta^{\sigma}}=\bigcap_{j=0}^{3} \frac{L_{j} \cap \Delta}{\Delta^{\sigma}} .
$$


Hence,

$$
T_{\gamma}(\Sigma \circ \widetilde{\Sigma})=\bigcap_{j, k=0,1} T_{\gamma}\left(C_{j} \circ \widetilde{C}_{k}\right)
$$

Similarly, any intersections from $\left\{C_{j} \circ C_{k}, j \cdot k=0,1\right\}$ intersect cleanly. This proves that $\left\{C_{j} \circ C_{k}, j, k=0,1\right\}$ is a cleanly system.

\subsubsection{Geometric method for weak compositions}

In this section we use geometric characterization of paired Lagrangian distributions and other distributions. The advantage of this approach is that we can treat many kinds of distributions in a united manner and, moreover, the geometrical condition of canonical relations appears naturally. However, the results which are obtained by this method are far from a symbolic calculus. The basic idea in this section is taken from [Jos99]. Now we recall some basic definitions:

Definition 2.22. A defining class of symbols on a manifold $X$ is a submodule of the spaces of homogeneous smooth functions of order one over the ring of those of order zero which is closed under Poisson bracket.

Definition 2.23. If $\mathcal{M}$ is a defining class of symbols, then $I^{(s)}(X, \mathcal{M})$ is the class of all $u \in H_{\mathrm{loc}}^{s}(X)$ such that

$$
P_{1} \ldots P_{k} u \in H_{\mathrm{loc}}^{s}(X)
$$

for all $k \in \mathbb{N}_{0}$ and $P_{j} \in \Psi_{\mathrm{cl}}^{1}(X)$ is a classical pseudo-differential operator whose principal symbol belongs to the class $\mathcal{M}$. Usually, we write

$$
I(X, \mathcal{M})=\bigcup_{s \in \mathbb{R}} I^{(s)}(X, \mathcal{M})
$$

Example 2.24. Let $\Lambda$ be a closed conic Lagrangian submanifold in $T^{*} X \backslash 0$ and $\mathcal{M}$ be the set of all smooth functions on $T^{*} X \backslash 0$ homogeneous of degree 1 which vanish on $\Lambda$. Then the class of Lagrangian distribution $I(X ; \Lambda)$ is determined by $I(X, \mathcal{M})$.

Example 2.25. Let $\left(\Lambda_{0}, \Lambda_{1}\right)$ be a cleanly intersecting pair of closed conic Lagrangian manifolds in $T^{*} X \backslash 0$. Then the class of paired Lagrangian distributions associated with $\Lambda_{0}, \Lambda_{1}$ is identified with $I(X, \mathcal{M})$, where $\mathcal{M}\left(\Lambda_{0}, \Lambda_{1}\right)$ is the set of all principal symbols of first order classical pseudo-differential operators on $X$ which vanish on $\Lambda_{0} \cup \Lambda_{1}$.

Similarly, we have: 
Definition 2.26. Let $L=\left\{\Lambda_{j}, 0 \leq j \leq \kappa\right\}$ be a system of closed, conic Lagrangian manifolds in $T^{*} X \backslash 0$. We denote $I(X ; L)=I(X, \mathcal{M}(L))$, where $\mathcal{M}(L)$ is the set of all first order symbols which vanish on $\bigcup_{j=0}^{\kappa} \Lambda_{j}$.

Proposition 2.27. Let $L=\left\{\Lambda_{j}, 0 \leq j \leq \kappa\right\}$ be a system of closed, conic Lagrangian manifolds in $T^{*} X \backslash 0$. If $u \in I(X ; L)$, then

$$
\mathrm{WF}(u) \subseteq \bigcup_{j=0}^{\kappa} \Lambda_{j} .
$$

Proof. Let $\left(x_{0}, \xi_{0}\right) \in T^{*} X \backslash\left(\cup_{j} \Lambda_{j}\right)$ and $\xi_{0} \neq 0$. We shall show that $\left(x_{0}, \xi_{0}\right) \notin \operatorname{WF}(u)$. Indeed, since $\left(x_{0}, \xi_{0}\right)$ does not belong to the closed conic subset $\cup \Lambda_{j}$, there exists an operator $P \in \Psi^{1}(X)$ such that $\sigma^{1}(P)=0$ on $\bigcup_{j} \Lambda_{j}$ and $\sigma^{1}(P)\left(x_{0}, \xi_{0}\right) \neq 0$. Hence, there is $s \in \mathbb{R}$ such that $P^{N} u \in H_{\text {loc }}^{s}(X)$ for all $N>0$. By micro-ellipticity (see [Hör97, Theorem 8.4.8]), we obtain $u \in H_{\mathrm{loc}}^{s+N}(X)$ microlocally at $\left(x_{0}, \xi_{0}\right)$ for all $N$. Therefore, $\left(x_{0}, \xi_{0}\right) \notin \mathrm{WF}(u)$.

Proposition 2.28. Let $J$ be a subset of $\{0,1, \ldots, \kappa\}$ and $\left(x_{0}, \xi_{0}\right) \notin \cup_{j \in J} \Lambda_{j}$. If $u \in I(X ; L)$ then

$$
u \in I\left(X ; \Lambda_{j}, j \notin J\right) \text { microlocally at }\left(x_{0}, \xi_{0}\right)
$$

Proof. Let $p(x, \xi)$ be a smooth function homogeneous of degree zero such that $p(x, \xi)=1$ in a conic neighbourhood $W$ of $\left(x_{0}, \xi_{0}\right)$, and vanishes on $\bigcup_{j \in J} L_{j}$. We have that $q$ satisfies $q-1 \in S^{-\infty}$ microlocally near $\left(x_{0}, \xi_{0}\right)$. Let $Q$ be a properly supported operator with the left full symbol $q(x, \xi)$. Choose a smooth function $\psi$ such that $\operatorname{supp} \psi \subset W$ and $\psi=1$ in a conic neighbourhood of $\left(x_{0}, \xi_{0}\right)$. Then we have $Q \psi=\psi$. Consider the following decomposition

$$
u=\psi(x, D) u+(u-\psi(x, D) u)
$$

Since $1-\psi \in S^{-\infty}$ near $\left(x_{0}, \xi_{0}\right)$, we obtain

$$
\left(x_{0}, \xi_{0}\right) \notin \mathrm{WF}((1-\psi(x, D)) u) .
$$

We shall verify that $\psi(x, D) u \in I\left(X ; \Lambda_{j}, j \notin J\right)$. Since $u \in I\left(X ; \Lambda_{j}\right)$, there exists a $s \in \mathbb{R}$ such that $u \in H_{\mathrm{loc}}^{s}(X)$. Hence, $v(x)=\psi(x, D) u \in H_{\mathrm{loc}}^{s}(X)$. Take any $P(x, D) \in$ $\operatorname{Op}\left(\mathcal{M}\left(\Lambda_{j}, j \notin J\right)\right)$. Then $\sigma(P \psi)=\sigma(P) \sigma(\psi)$ vanishes on $\bigcup_{j} \Lambda_{j}$. Thus, we obtain

$$
P v(x)=P \psi u \in H_{\mathrm{loc}}^{s}(X),
$$


as $P \psi \in \mathrm{Op}\left(\mathcal{M}\left(\Lambda_{j}\right)\right)$. Assume that

$$
P_{1} \ldots P_{N} v \in H_{\mathrm{loc}}^{s}(X), \quad \forall \sigma\left(P_{1}\right), \ldots, \sigma\left(P_{N}\right) \in \mathcal{M}\left(\Lambda_{j}, j \notin J\right)
$$

Take any $N+1$ symbols $p_{j}, j=1, \ldots, N+1$, we have

$$
\begin{aligned}
P_{1} \ldots P_{N} P_{N+1} v & =P_{1} \ldots P_{N} P_{N+1} \psi u \\
& =P_{1} \ldots P_{N} P_{N+1} Q^{N+1} \psi u \\
& =P_{1} \ldots P_{N} Q^{N}\left(P_{N+1} Q\right) \psi u+P_{1} \ldots\left(P_{N}\left[P_{N+1}, Q^{N}\right] Q\right) \psi u
\end{aligned}
$$

The second term belongs to $H_{\mathrm{loc}}^{s}(X)$ by the inductive assumption. For the first term, using commutator method, one easily checks that

$$
P_{1} \ldots P_{N} Q^{N} \in \mathrm{Op}\left(\mathcal{M}\left(\Lambda_{j}, 0 \leq j \leq \kappa\right)\right)^{N}
$$

Hence,

$$
P_{1} \ldots P_{N} Q^{N}\left(P_{N+1} Q\right) \psi u \in H_{\mathrm{loc}}^{s}(X) .
$$

Therefore, $\psi(x, D) u \in I\left(X ; \Lambda_{j}, j \notin J\right)$.

Abstract theory of distributions states that the kernel of the composition can be decomposed into three fundamental operations: tensor product, pull-back, and push-forward. Namely, let $A: \mathcal{D}(Z) \rightarrow \mathcal{D}^{\prime}(Y)$ and $B: \mathcal{D}(Y) \rightarrow \mathcal{D}^{\prime}(X)$ be such that $\mathrm{WF}_{Y}(A) \cap W F_{Y}^{\prime}(B)=\emptyset$ and the projection $\operatorname{supp} k_{A} \times \operatorname{supp} k_{B}$ into $X \times Z$ is proper. Then $A \circ B$ is well-defined as an operator from $\mathcal{D}(Z)$ to $\mathcal{D}^{\prime}(X)$ and $k_{A \circ B}=\pi_{*} \Delta^{*}\left(k_{A} \otimes k_{B}\right)$, where $\Delta$ is the diagonal map $X \times Y \times Z \rightarrow X \times Y \times Y \times Z$ and $\pi: X \times Y \times Z \rightarrow X \times Z$. Therefore, one needs to understand the change of defining classes of symbols under these operations.

Theorem 2.29 (Joshi). Let $\mathcal{M}$ be a defining class of symbols on $X \times Y$ where $X, Y$ are smooth manifolds and $Y$ is equipped with a smooth density. If $u \in I^{(s)}(X \times Y, \mathcal{M})$ and the support of $u$ is proper with respect to the projection onto $Y$, then $\pi_{*} u \in I^{(s)}(X, \widetilde{\mathcal{M}})$, where $\widetilde{\mathcal{M}}$ is the class of symbols on $X$, which after being pulled back to $\bigcup_{x \in X} N_{X \times Y}^{*}(\{x\} \times Y)$ are restrictions of elements of $\mathcal{M}$.

Remark 2.30. The operator $\pi_{*} \Delta^{*}$ is a Fourier integral operator associated with the canonical relation $\Gamma=\{(x, \xi, z, \zeta, x, \xi, y, \eta, y,-\eta, z, \zeta)\}$ and its principal symbol is one. Therefore, in composition, only $\mathrm{WF}\left(k_{A} \otimes k_{B}\right)$ near $(x, \xi, y, \eta, y, \eta, z, \zeta)$ contributes to the wave front set of the composite. For more details, see [BG81, Theorem 9.4]. 
We now provide the decomposition of the tensor product of paired Lagrangian distributions. Roughly speaking, one proves that on the regular part, the tensor product $I\left(\Lambda_{0}, \Lambda_{1}\right) \otimes I\left(\widetilde{\Lambda}_{0}, \widetilde{\Lambda}_{1}\right)$ of two classes of paired Lagrangian distributions is characterized by the class of first order symbols which vanish on $\Lambda_{j} \times \widetilde{\Lambda}_{k} \mid j, k=0,1$. The following argument relies on the proof for the tensor product of two isotropic distributions which was given in [BG81, Theorem 9.3].

Lemma 2.31. Let $\left(\Lambda_{0}, \Lambda_{1}\right)$ and $\left(\tilde{\Lambda}_{0}, \tilde{\Lambda}_{1}\right)$ be cleanly intersecting pairs of closed, conic Lagrangian manifolds in $T^{*} X \backslash 0$ and $T^{*} Y \backslash 0$, respectively. Assume that $u(x) \in I\left(\Lambda_{0}, \Lambda_{1}\right)$ and $v(y) \in I\left(\tilde{\Lambda}_{0}, \tilde{\Lambda}_{1}\right)$ are paired Lagrangian distributions and $U$ is a conic neighbourhood of the conic set

$$
\bigcup_{j=0,1}\left(\Lambda_{j} \times 0_{Y}\right) \bigcup_{k=0,1}\left(0_{X} \times \tilde{\Lambda}_{k}\right)
$$

Then there exists a decomposition

$$
u \otimes v=w+r
$$

where $w \in I\left(\Lambda_{j} \times \tilde{\Lambda}_{k}, j, k=0,1\right)$ and $\mathrm{WF}(r) \subseteq U$.

Proof. Because the statement is invariant under conjugating by elliptic FIOs associated with canonical transformation, without loss of generality, one takes $X=\mathbb{R}^{d_{1}}, Y=\mathbb{R}^{d_{2}}$ and

$$
\begin{array}{ll}
\Lambda_{0}=N^{*}\{x=0\}, & \Lambda_{1}=N^{*}\left\{x^{\prime \prime}=0\right\}, \\
\tilde{\Lambda}_{0}=N^{*}\{y=0\}, & \tilde{\Lambda}_{1}=N^{*}\left\{y^{\prime \prime}=0\right\} .
\end{array}
$$

Hence, paired Lagrangian distributions $u, v$ have the following representations:

$$
\begin{aligned}
& u(x)=\int e^{i x \xi} a(x, \xi) d \xi \\
& v(y)=\int e^{i y \eta} b(y, \eta) d \eta
\end{aligned}
$$

where smooth functions $a(x, \xi)$ and $b(y, \eta)$ satisfy the following estimates:

$$
\begin{aligned}
& \left|D_{\xi}^{\alpha} D_{x}^{\beta} a(x, \xi)\right| \leq C\left\langle\xi^{\prime}\right\rangle^{m_{0}-\left|\alpha^{\prime}\right|}\langle\xi\rangle^{m_{1}-\left|\alpha^{\prime \prime}\right|}, x \in K \Subset X \\
& \left|D_{\eta}^{\alpha} D_{y}^{\beta} b(y, \eta)\right| \leq C^{\prime}\left\langle\eta^{\prime}\right\rangle^{p_{0}-\left|\alpha^{\prime}\right|}\langle\eta\rangle^{p_{1}-\left|\alpha^{\prime \prime}\right|}, x \in K^{\prime} \Subset Y .
\end{aligned}
$$

This implies that

$$
(u \otimes v)(x, y)=\iint e^{i(x \xi+y \eta)} a(x, \xi) b(y, \eta) d \xi d \eta
$$


For any positive constant $\epsilon<1$, choose a cut-off function $\chi \in C_{c}^{\infty}(\mathbb{R})$ such that $\chi(t)=1$ if $\epsilon \leq t \leq \epsilon^{-1}$. Define $w(x, y)=\iint e^{i(x \xi+y \eta)} \chi\left(\frac{\langle\eta\rangle}{\langle\xi\rangle}\right) a(x, \xi) b(y, \eta) d \xi d \eta$ and $r=u-w$.

We shall show that this decomposition has the desired properties if $\epsilon$ is sufficiently small. Indeed, in the support of $\chi$ one has $\langle\eta\rangle \simeq\langle\xi\rangle$. Therefore, $c(x, y, \xi, \eta)=\chi a b$ satisfies the following estimate:

$$
\left|D_{\xi}^{\alpha} D_{\eta}^{\beta} D_{x, y}^{\gamma} c(x, y, \xi, \eta)\right| \leq C\left\langle\xi^{\prime}\right\rangle^{m_{0}-\left|\alpha^{\prime}\right|}\left\langle\eta^{\prime}\right\rangle^{p_{0}-\left|\beta^{\prime}\right|}\langle\xi, \eta\rangle^{m_{1}+p_{1}-\left|\alpha^{\prime \prime}\right|-\left|\beta^{\prime \prime}\right|},(x, y) \in K \Subset X \times Y .
$$

This yields that $w \in I\left(\Lambda_{j} \times \tilde{\Lambda}_{k}, j, k=0,1\right)$. It remains to prove that $\mathrm{WF}(u-w) \subset U$. It follows from the facts that $\operatorname{WF}(u-w) \subseteq \bigcup_{j, k=0,1}\left(\Lambda_{j} \times \tilde{\Lambda}_{k} \cap \Lambda_{j} \times 0_{Y} \cap 0_{X} \times \tilde{\Lambda}_{k}\right)$ and $\mathrm{WF}(u-w)$ does not meet the set $\left\{(x, y, \xi, \eta) \mid \epsilon \leq \frac{\langle\eta\rangle}{\langle\xi\rangle} \leq \epsilon^{-1}\right\}$.

Now we are in a position to state the main result in this section, the weak composition of paired Lagrangian distributions.

Theorem 2.32. Let $C_{0}, C_{1}$ and $\widetilde{C}_{0}, \widetilde{C}_{1}$ be clean pairs of canonical relations from $T^{*} Y \backslash 0$ to $T^{*} X \backslash 0$ and from $T^{*} Z \backslash 0$ to $T^{*} Y \backslash 0$, respectively. Define

$$
\begin{aligned}
& D=\left(T^{*} X \backslash 0\right) \times \Delta_{T^{*} Y \backslash 0} \times\left(T^{*} Z \backslash 0\right), \\
& L_{i}=C_{j} \times \widetilde{C}_{k}, j, k=0,1, i=2 j+k .
\end{aligned}
$$

Assume that the following assumptions are fulfilled.

1. All systems $\left(D, L_{0}, L_{1}\right) ;\left(D, L_{0}, L_{2}\right) ;\left(D, L_{1}, L_{3}\right) ;\left(D, L_{2}, L_{3}\right)$ are cleanly intersecting triples.

2. The system $\left(D \cap L_{j}, j=0,1,2,3\right)$ forms a CIS of four manifolds.

3. The rank condition

$$
T_{p} D \bigcap \sum_{j=0}^{3} T_{p} L_{j}=\sum_{j=0}^{3} T_{p}\left(D \cap L_{j}\right), \quad p \in D \bigcap_{j} L_{j} .
$$

Then $\left\{C_{j} \circ \widetilde{C}_{k}, j, k=0,1\right\}$ is a system of canonical relations satisfying:

$$
\begin{aligned}
T_{\gamma}\left(\left(C_{j} \circ \widetilde{C}_{0}\right) \cap\left(C_{j} \circ \widetilde{C}_{1}\right)\right) & =T_{\gamma}\left(C_{j} \circ \widetilde{C}_{0}\right) \cap T_{\gamma}\left(C_{j} \circ \widetilde{C}_{1}\right), & & \gamma \in C_{j} \circ\left(\widetilde{C}_{0} \cap \widetilde{C}_{1}\right), j=0,1 ; \\
T_{\gamma}\left(\left(C_{0} \circ \widetilde{C}_{k}\right) \cap\left(C_{1} \circ \widetilde{C}_{k}\right)\right) & =T_{\gamma}\left(C_{0} \circ \widetilde{C}_{k}\right) \cap T_{\gamma}\left(C_{1} \circ \widetilde{C}_{k}\right), & & \gamma \in\left(C_{0} \cap C_{1}\right) \circ \widetilde{C}_{k}, k=0,1 ; \\
T_{\gamma}\left(\bigcap_{j, k=0,1} C_{j} \circ \widetilde{C}_{k}\right) & =\bigcap_{j, k=0,1} T_{\gamma}\left(C_{j} \circ \widetilde{C}_{k}\right), & & \gamma \in\left(C_{0} \cap C_{1}\right) \circ\left(\widetilde{C}_{0} \cap \widetilde{C}_{1}\right) .
\end{aligned}
$$

Moreover, the weak composition law holds:

$$
I\left(C_{0}, C_{1}\right) \circ I\left(\widetilde{C}_{0}, \widetilde{C}_{1}\right) \subseteq I\left(C_{j} \circ \widetilde{C}_{k}, j, k=0,1\right) .
$$


Proof. Because of the cleanness assumption, $\left(C_{j} \circ C_{k}, j, k=0,1\right)$ contains only canonical relations from $T^{*} Z \backslash 0$ into $T^{*} X \backslash 0$. The geometric part follows from Lemma 2.21 .

Now we prove the analytic part. By a result proved by Joshi [Jos99], the composite $A \circ B$ belongs to the class of distributions which is determined by the defining class of symbol $\mathcal{M}$ on $T^{*}(X \times Z) \backslash 0$, after being pulled back to $D=\left(T^{*} X \backslash 0\right) \times \Delta_{T^{*} Y \backslash 0} \times\left(T^{*} Z \backslash 0\right)$ are restriction of class of symbols vanishing on $C_{j} \times \widetilde{C}_{k}$. Hence, we only need to show that the set of all first order principal symbols vanishing on $\cup C_{j} \circ \widetilde{C}_{k}$ is the stable defining class of composed operators. It is equivalent to prove that any smooth function on $D$ homogeneous of degree 1 which vanishes on $\bigcup_{j=0}^{3} L_{j} \cap D$ can be extended to a smooth function on $T^{*}(X \times Y \times Y \times Z) \backslash 0$ homogeneous of degree 1 which vanishes on $\bigcup_{j=0}^{3} L_{j}$. The hard part in the proof is the LIFT property since the system $\left(D, L_{j}, j=0,1,2,3\right)$ is not a CIS. Since $T^{*}(X \times Y \times Y \times Z) \backslash 0$ is a conic manifold, the space of $\mathbb{R}_{+}$-orbits, denoted by $S$, is a smooth manifold. Furthermore, the canonical projection $\pi$ is a fibre map. Note that $\pi(D), \pi\left(L_{j}\right), j=0,1,2,3$ are smooth submanifolds of $S$.

By the argument in the proof of Lemma 2.19 , the proof follows from the lift lemma.

Lemma 2.33 (Lift lemma). The submanifold $\pi(D)$ of $S$ has the lift property with respect to $\bigcup_{j=0}^{3} \pi\left(L_{j}\right)$.

Proof of lift lemma. It suffices to prove the statement locally. Note that the projection $\pi$ commutes with the intersections of conic submanifolds. Since the action is free and proper, $\pi(D)$ is an embedded submanifold of $S$. Suppose that $\pi(D)$ is given by coordinates $\pi(D)=\{(x, y) \mid y=0\}$. By Lemma 2.18, the intersections $\pi\left(D \cap L_{j}\right), j=0,1,2,3$ form a CIS. Hence, there are local coordinates $x_{\mu}$ which are numbered by "subsets" of $J=$ $\{0,1,2,3\}$ :

$$
\begin{aligned}
\pi\left(D \cap L_{j}\right) & =\left\{x_{\mu}=0, \mu \in I_{j}\right\}, \quad j=0,1,2,3, \\
I_{j} & =\{\mu \subseteq\{0,1,2,3\} \mid j \in \mu\},
\end{aligned}
$$

where $x=\left(x_{\mu}, \bar{x}\right), \mu \subseteq\{0,1,2,3\}$ are local coordinates. For example, $\pi\left(D \cap L_{0}\right)$ is given by the following coordinates

$$
x_{0}, x_{01}, x_{02}, x_{03}, x_{012}, x_{013}, x_{023}, x_{0123} \text {, }
$$

while $\pi\left(D \cap L_{1}\right)$ is given by the vanishing of

$$
x_{1}, x_{10}, x_{12}, x_{13}, x_{012}, x_{013}, x_{123}, x_{0123} \text {. }
$$


Then the set $\mathcal{M}$ of all smooth functions on $\pi(D)$ vanishing on $\bigcup_{j=0}^{3}\left(\pi(D) \cap \pi\left(L_{j}\right)\right)$ is a finitely generated ideal, whose generators are given by

$$
\begin{array}{ll}
x_{0123}, & \\
x_{\mu_{1}} x_{\mu_{2}}, & \mu_{1} \neq J, \mu_{2} \neq J, \mu_{1} \cup \mu_{2}=J, \\
x_{\mu_{1}} x_{\mu_{2}} x_{\mu_{3}}, & \mu_{1} \cup \mu_{2} \neq J, \mu_{2} \cup \mu_{3} \neq J, \mu_{3} \cup \mu_{1} \neq J, \mu_{1} \cup \mu_{2} \cup \mu_{3}=J, \\
x_{1} x_{2} x_{3} x_{4} . &
\end{array}
$$

Because of the appearance of the full coordinates, the generators of $\mathcal{M}$, in general, are quite complicated. Since in our case, $D \cap L_{j}$ has special properties, a simpler form of local coordinates holds.

FACT 1. With the notation above, the coordinates $\left(x_{0}, x_{1}, x_{2}, x_{3}, x_{12}, x_{03}\right)$ are absent from vanishing coordinates, that is,

$$
\begin{aligned}
& \pi\left(D \cap L_{0}\right)=\left\{x_{01}, x_{02}, x_{023}, x_{013}, x_{012}, x_{0123}\right\} \\
& \pi\left(D \cap L_{1}\right)=\left\{x_{10}, x_{13}, x_{123}, x_{013}, x_{012}, x_{0123}\right\} \\
& \pi\left(D \cap L_{2}\right)=\left\{x_{02}, x_{23}, x_{123}, x_{023}, x_{012}, x_{0123}\right\} \\
& \pi\left(D \cap L_{3}\right)=\left\{x_{13}, x_{23}, x_{123}, x_{023}, x_{013}, x_{0123}\right\} .
\end{aligned}
$$

Indeed, we shall show that $\#\left(x_{0}, x_{03}\right)=0$. For any $p \in D \cap L_{0} \cap L_{1} \cap L_{2}$, one has

$$
T_{p}\left(L_{1} \cap L_{2}\right)=T_{p}\left(L_{0} \cap L_{1} \cap L_{2}\right) \Longleftrightarrow T_{p} L_{1}+T_{p} L_{2}=T_{p} L_{0}+T_{p} L_{1}+T_{p} L_{2} .
$$

Adding $T_{p} D^{\sigma}$ to both sides of this equation and taking the symplectic complements, one obtains

$$
T_{p} D_{p} \cap T_{p} L_{1} \cap T_{p} L_{2}=T_{p} D \cap T_{p} L_{0} \cap T_{p} L_{1} \cap T_{p} L_{2} .
$$

This yields $T_{p}\left(D \cap L_{1} \cap L_{2}\right)=T_{p}\left(D \cap L_{0} \cap L_{1} \cap L_{2}\right)$ by CIS assumption of $D \cap L_{j}, j=0,1,2$. Using the following relations

$$
\begin{gathered}
\operatorname{dim} \pi\left(D \cap L_{1} \cap L_{2}\right)=\operatorname{dim} D \cap L_{1} \cap L_{2}-1, \\
\operatorname{dim} \pi\left(D \cap L_{0} \cap L_{1} \cap L_{2}\right)=\operatorname{dim} D \cap L_{0} \cap L_{1} \cap L_{2}-1, \\
\operatorname{dim} T_{\pi(p)}\left(\pi\left(D \cap L_{1} \cap L_{2}\right)-\operatorname{dim} T_{\pi(p)}\left(\pi\left(D \cap L_{0} \cap L_{1} \cap L_{2}\right)=\#\left(x_{0}, x_{03}\right),\right.\right.
\end{gathered}
$$

one obtains the conclusion above.

This leads to the following very useful statement: 
FACT 2. The set $\mathcal{M} / \operatorname{span}\left(x_{0123}\right)$ is determined by

$$
\operatorname{span}\left\langle x_{01} x_{23}, x_{02} x_{13}, x_{\hat{0}} x_{01}, x_{\hat{0}} x_{02}, x_{\hat{1}} x_{01}, x_{\hat{1}} x_{13}, x_{\hat{2}} x_{20}, x_{\hat{2}} x_{23}, x_{\hat{3}} x_{31}, x_{\hat{3}} x_{32}, x_{\hat{j}} x_{\hat{k}}\right\rangle,
$$

where $j \neq k, j, k=0,1,2,3, \hat{j}=\{0,1,2,3\} \backslash\{j\}$ for $j=0,1,2,3$.

As a consequence, any function $f \in \mathcal{M} / \operatorname{span}\left(x_{0123}\right)$ can be decomposed into $g(x) h(x)$ such that $g$ and $h$ vanish on $\pi\left(D \cap L_{0}\right) \cup \pi\left(D \cap L_{1}\right)$ and $\pi\left(D \cap L_{1}\right) \cup \pi\left(D \cap L_{3}\right)$ respectively, or on $\pi\left(D \cap L_{0}\right) \cup \pi\left(D \cap L_{1}\right)$ and $\pi\left(D \cap L_{1}\right) \cup \pi\left(D \cap L_{3}\right)$ respectively. By cleanly intersecting triples, one defines $\tilde{f}(x, y)=\tilde{g}(x, y) \tilde{h}(x, y)$, where $\tilde{g}, \tilde{h}$ are the corresponding extensions of $g, h$ too. This fact implies the following result:

FACT 3. The set $\mathcal{M} / \operatorname{span}\left(x_{0123}\right)$ has the lift property.

Therefore, it is enough to show that $x_{0123}$ has some extension on $S$ that vanishes on $\bigcup \pi\left(L_{j}\right), j=0,1,2,3$.

With these notations, it is apparent that for all $\mu \subset J$, the dimension of the space

$$
\sum_{j \in \mu} T_{p} \pi\left(D \cap L_{j}\right), \quad p \in \pi(D) \bigcap_{j \in \mu} \pi\left(L_{j}\right)
$$

is equal to

$$
\operatorname{dim} \pi(D)-\sum_{\mu \subseteq v} \#\left(x_{v}\right)
$$

Hence, the numbers of variables $x_{\mu}, \mu \subseteq J$ can be computed in terms of the dimensions of those spaces $T_{p} \pi\left(D \cap L_{j}\right)$. For instance, the space $\sum_{j=0}^{3} T_{p} \pi\left(D \cap L_{j}\right)$ has dimension $\operatorname{dim} \pi(D)-\#\left(x_{0123}\right)$. Similarly, $\left(\pi\left(L_{0}\right), \pi\left(L_{1}\right), \pi\left(L_{2}\right), \pi\left(L_{3}\right)\right)$ is a CIS by Lemma 2.18 . Thus, there exist coordinates $f_{\mu}, \mu \subseteq J$ such that $\pi\left(L_{j}\right)$ is given by the following coordinates vanishing:

$$
\pi\left(L_{j}\right)=\left\{f_{\mu}(x, y)=0, \mu \subseteq J, j \in \mu\right\} .
$$

Thus, the space $T_{p} \pi(D) \cap \sum_{j=0}^{3} T_{p} \pi\left(L_{j}\right)$ is determined by the following equations:

$$
\begin{aligned}
\left(x, y ; v_{x}, v_{y}\right) & \in T_{p} \pi(D) \mid y=0, x \in \cap \pi\left(D \cap L_{j}\right), t_{y}=0 \\
\left(v_{x}, v_{y}\right) & \in \sum_{j=0}^{3} T_{p} \pi\left(L_{j}\right)\left|d_{x} f_{0123}(x, y) v_{x}+d_{y} f_{0123}(x, y) v_{y}\right|_{y=0}=0 .
\end{aligned}
$$

Hence, setting $g_{0123}=\left.f_{0123}\right|_{\pi(D)}$, we conclude that

$$
\operatorname{dim}\left(T_{p} \pi(D) \bigcap \sum_{j=0}^{3} T_{p} \pi\left(L_{j}\right)\right)=\operatorname{dim} \pi(D)-\operatorname{rank}\left\{d g_{0123}\right\}
$$


On the other hand, we have

$$
\begin{aligned}
\operatorname{dim}\left(\sum_{j=0}^{3} T_{p} \pi\left(D \cap L_{j}\right)\right) & =\operatorname{dim} \sum_{j=0}^{3} T_{p}\left(D \cap L_{j}\right)-1, \\
\operatorname{dim}\left(T_{p} \pi(D) \bigcap \sum_{j=0}^{3} T_{p} \pi\left(L_{j}\right)\right) & =\operatorname{dim}\left(T_{p} D \bigcap \sum_{j=0}^{3} T_{p} L_{j}\right)-1 .
\end{aligned}
$$

Therefore, condition (2.3II) is equivalent to

$$
\operatorname{rank}\left\{d g_{0123}\right\}=\operatorname{rank}\left(d x_{0123}\right)
$$

Because $g_{0123}$ vanishes on $\bigcup_{j=0}^{3} \pi\left(D \cap L_{j}\right)$, there exists a smooth matrix $C(x)$ satisfying

$$
g_{0123}(x)=C(x) x_{0123}+R,
$$

where

$$
R=\operatorname{span}\left\{x_{\mu_{1}} x_{\mu_{2}} ; x_{\mu_{1}} x_{\mu_{2}} x_{\mu_{3}} ; x_{1} x_{2} x_{3} x_{4}\right\}=o\left(x^{2}\right)
$$

This implies that $\operatorname{rank} C(p)=\operatorname{rank}\left\{d g_{0123}\right\}$. So we can choose a left inverse $B$ of $C$ and extend it into a neighbourhood of $p$ in the whole manifold. And the formula above can be written as

$$
x_{0123}=B g_{0123}-B R \text {. }
$$

We have to show that the functions $x_{0123}$ can be extended in to smooth functions on $S$ which vanish on $\bigcup \pi\left(L_{j}\right)$. One already has that property holds for functions $g_{0123}$ trivially and for $R(x)$ by the fact above. The proof is completed.

Remark 2.34. The proof strongly relies on the special property of the system $L_{0}, L_{1}, L_{2}, L_{2}$. The analogous method can be used to prove the composition of a FIO $A \in I(X, Y ; C)$ with a class of distributions associated with a CIS of four Lagrangian manifolds $L_{0}, L_{1}, L_{2}, L_{3}$ of $T^{*} Y \backslash 0$. In this case, one needs further assumption.

Example 2.35. Let consider the following canonical relations:

$$
\begin{array}{ll}
C_{0}=N^{*}\{x-y=0\}^{\prime}, & C_{1}=N^{*}\left\{x^{\prime \prime}-y^{\prime \prime}=0, x^{\prime \prime \prime}-y^{\prime \prime \prime}=0\right\}^{\prime}, \\
\widetilde{C}_{0}=N^{*}\{y-z=0\}^{\prime}, & \widetilde{C}_{1}=N^{*}\left\{y^{\prime}-z^{\prime}=0, y^{\prime \prime \prime}-z^{\prime \prime \prime}=0\right\}^{\prime} .
\end{array}
$$


Let $D=\{y-\tilde{y}=0, \eta-\tilde{\eta}=0\}$ with standard coordinates $(x, y, \tilde{y}, z ; \xi, \eta, \tilde{\eta}, \zeta)$ on $T^{*}(X \times$ $Y \times Y \times Z$ ). With the notations as in Theorem [2.32], we have

$$
\begin{aligned}
& L_{0}=N^{*}\{x-y=0, \tilde{y}-z=0\}^{\prime}, \\
& L_{1}=N^{*}\left\{x-y=0, \tilde{y}^{\prime}-z^{\prime}=0, \tilde{y}^{\prime \prime \prime}-z^{\prime \prime \prime}=0\right\}^{\prime}, \\
& L_{2}=N^{*}\left\{x^{\prime \prime}-y^{\prime \prime}=0, x^{\prime \prime \prime}-y^{\prime \prime \prime}=0, \tilde{y}^{\prime}-z^{\prime}=0, \tilde{y}^{\prime \prime \prime}-z^{\prime \prime \prime}=0\right\}^{\prime}, \\
& L_{3}=N^{*}\left\{x^{\prime \prime}-y^{\prime \prime}=0, x^{\prime \prime \prime}-y^{\prime \prime \prime}=0, \tilde{y}^{\prime}-z^{\prime}=0, \tilde{y}^{\prime \prime \prime}-z^{\prime \prime \prime}=0\right\}^{\prime}, \\
& D=\{y-\tilde{y}=0, \eta-\tilde{\eta}=0\} .
\end{aligned}
$$

Obviously, the system $\left\{D, L_{0}, L_{1}, L_{2}, L_{3}\right\}$ forms a CIS. Hence, the weak composition law holds:

$$
I\left(C_{0}, C_{1}\right) \circ I\left(\widetilde{C}_{0}, \widetilde{C}_{1}\right) \subseteq I\left(E_{0}, E_{1}, E_{2}, E_{3}\right),
$$

where $E_{2 j+k}=C_{j} \circ \widetilde{C}_{k}, j, k=0,1$ are four canonical relations from $T^{*} Z \backslash 0$ into $T^{*} Z \backslash 0$.

Example 2.36. Consider the following canonical relations

$$
\begin{aligned}
& C_{0}=N^{*}\{x-y=0\}^{\prime}, \quad C_{1}=N^{*}\left\{x^{\prime}-y^{\prime}=0, x^{\prime \prime \prime}-y^{\prime \prime \prime}=0\right\}^{\prime}, \\
& \widetilde{C}_{0}=N^{*}\{y-z=0\}^{\prime}, \quad \widetilde{C}_{1}=N^{*}\left\{y^{\prime \prime \prime}-z^{\prime \prime \prime}=0\right\}^{\prime}, \\
& E_{0}=N^{*}\{x-z=0\}^{\prime}, \quad E_{1}=N^{*}\left\{x^{\prime \prime \prime}-z^{\prime \prime \prime}=0\right\}^{\prime}, \quad E_{2}=N^{*}\left\{x^{\prime}-z^{\prime}=0, x^{\prime \prime \prime}-z^{\prime \prime \prime}=0\right\}^{\prime}
\end{aligned}
$$

It is not hard to verify that all assumptions in the previous theorem are fulfilled. Moreover,

$$
C_{0} \circ \widetilde{C}_{0}=E_{0}, C_{0} \circ \widetilde{C}_{1}=E_{1}, C_{1} \circ \widetilde{C}_{0}=E_{2}, C_{1} \circ \widetilde{C}_{1}=E_{1} .
$$

Therefore, one has the weak composition law

$$
I\left(C_{0}, C_{1}\right) \circ I\left(\widetilde{C}_{0}, \widetilde{C}_{1}\right) \subseteq I\left(E_{0}, E_{1}, E_{2}\right)
$$

where the left hand side is the space of operators whose Schwartz kernels correspond to three Lagrangian manifolds.

Example 2.37. Consider the following canonical relations

$$
\begin{array}{ll}
C_{0}=N^{*}\left\{x^{\prime}-y^{\prime}=0, x^{\prime \prime \prime}-y^{\prime \prime \prime}=0\right\}^{\prime}, & C_{1}=N^{*}\left\{x^{\prime \prime \prime}-y^{\prime \prime \prime}=0\right\}^{\prime} \\
\widetilde{C}_{0}=N^{*}\left\{y^{\prime \prime}-z^{\prime \prime}=0, x^{\prime \prime \prime}-z^{\prime \prime \prime}=0\right\}^{\prime}, & \widetilde{C}_{1}=N^{*}\left\{y^{\prime \prime \prime}-z^{\prime \prime \prime}=0\right\}^{\prime} \\
E_{0}=N^{*}\left\{x^{\prime \prime \prime}-z^{\prime \prime \prime}=0\right\}^{\prime} . &
\end{array}
$$


All assumptions in Theorem 2.32 are satisfied. In addition,

$$
C_{j} \circ \widetilde{C}_{k}=E_{0}, j, k=0,1 .
$$

Hence, the weak composition law holds

$$
I\left(C_{0}, C_{1}\right) \circ I\left(\widetilde{C}_{0}, \widetilde{C}_{1}\right) \subseteq I\left(E_{0}\right) .
$$

Corollary 2.38. Assume that all assumptions in Theorem 2.32 are fulfilled and the system $\left\{C_{j} \circ \widetilde{C}_{k}, j, k=0,1\right\}$ contains two canonical relations $E_{0}, E_{1}$.

Case 1. When the following compatible condition is satisfied

$$
\left(C_{0} \cap C_{1}\right) \circ\left(\widetilde{C}_{0} \cap \widetilde{C}_{1}\right)=E_{0} \cap E_{1},
$$

then $E_{0}, E_{1}$ intersect cleanly. Moreover, the weak composition holds:

$$
I\left(C_{0}, C_{1}\right) \circ I\left(\widetilde{C}_{0}, \widetilde{C}_{1}\right) \subseteq I\left(E_{0}, E_{1}\right) .
$$

Case 2. When $E_{0}$ and $E_{1}$ intersects cleanly and $K:=\left(C_{0} \cap C_{1}\right) \circ\left(\widetilde{C}_{0} \cap \widetilde{C}_{1}\right) \subsetneq E_{0} \cap E_{1}$, the weak composition holds

$$
I\left(C_{0}, C_{1}\right) \circ I\left(\widetilde{C}_{0}, \widetilde{C}_{1}\right) \subseteq I\left(E_{0}, K\right)+I\left(E_{1}, K\right) \subsetneq I\left(E_{0}, E_{1}\right) .
$$

Proof. Obviously, when $\left\{C_{j} \circ \widetilde{C}_{k}, j, k=0,1\right\}$ contains only two cleanly intersecting canonical relations $E_{0}, E_{1}$ then $I\left(C_{j} \circ \widetilde{C}_{k}, j, k=0,1\right)=I\left(E_{0}, E_{1}\right)$.

Let us consider the second case, when $\left\{C_{j} \circ \widetilde{C}_{k}, j, k=0,1\right\}$ has two canonical relations $E_{0}, E_{1}$ satisfying $\left(C_{0} \cap C_{1}\right) \circ\left(\widetilde{C}_{0} \cap \widetilde{C}_{1}\right)=E_{0} \cap E_{1}$. Because of the composition of isotropic relations, Theorem L.4, the set $\Sigma=\left(C_{0} \cap C_{1}\right) \circ\left(\widetilde{C}_{0} \cap \widetilde{C}_{1}\right)$ is an isotropic manifold. Thus we need to verify that for all $\gamma \in \Sigma=E_{0} \cap E_{1}$, the tangent condition holds. Indeed, due to relation (2.3I), for any $p$ in the fibre $\pi^{-1}(\gamma)$ we have

$$
T_{p} D \cap \sum T_{p}\left(C_{j} \times \widetilde{C}_{k}\right)=\sum T_{p}\left(D \cap\left(C_{j} \times \widetilde{C}_{k}\right) .\right.
$$

Taking the symplectic complement and intersecting with $T_{p} D^{\sigma}$, we obtain

$$
T_{p}\left(\left(C_{0} \cap C_{1}\right) \times\left(\widetilde{C}_{0} \times \widetilde{C}_{1}\right) \bigcap D\right)+T_{p} D^{\sigma}=\bigcap\left(T_{p}\left(D \cap C_{j} \circ \widetilde{C}_{k}\right)+T_{p} D^{\sigma}\right)
$$

Dividing by $T_{p} \Delta^{\sigma}$, this yields

$$
T_{\gamma} \Sigma=\bigcap T_{\gamma}\left(C_{j} \circ \widetilde{C}_{k}\right)
$$

Replacing $C_{j} \circ \widetilde{C}_{k}$ by $E_{0}, E_{1}$, one concludes that $T_{\gamma}\left(E_{0} \cap E_{1}\right)=T_{\gamma} E_{0} \cap T_{\gamma} E_{1}$. Hence, $E_{0}$ and $E_{1}$ intersect cleanly. The proof is completed. 
Example 2.39. Let $X=Y=Z=\mathbb{R}^{d}$ with coordinates $x=\left(x^{\prime}, x^{\prime \prime}\right) \in \mathbb{R}^{d^{\prime}+d^{\prime \prime}}$. Consider the following canonical relations:

$$
\begin{array}{ll}
C_{0}=N^{*}\{x-y=0\}^{\prime}, & C_{1}=N^{*}\left\{x^{\prime}-y^{\prime}=0\right\}^{\prime}, \\
\widetilde{C}_{0}=N^{*}\{y-z=0\}^{\prime}, & \widetilde{C}_{1}=N^{*}\left\{y^{\prime}-z^{\prime}=0\right\}^{\prime}, \\
E_{0}=N^{*}\{x-z=0\}^{\prime}, & E_{1}=N^{*}\left\{x^{\prime}-z^{\prime}=0\right\}^{\prime} .
\end{array}
$$

Then these canonical relations satisfy all assumptions in Corollary 2.38. Moreover,

$$
\begin{aligned}
C_{0} \circ \widetilde{C}_{0} & =E_{0}, \\
C_{j} \circ \widetilde{C}_{k} & =E_{1}, \forall(j, k) \neq(0,0) \\
\left(C_{0} \cap C_{1}\right) \circ\left(\widetilde{C}_{0} \cap \widetilde{C}_{1}\right) & =E_{0} \cap E_{1} .
\end{aligned}
$$

Hence, we obtain the weak composition law

$$
I\left(C_{0}, C_{1}\right) \circ I\left(\widetilde{C}_{0}, \widetilde{C}_{1}\right) \subseteq I\left(E_{0}, E_{1}\right) .
$$

Indeed, $D, L_{0}, L_{1}, L_{2}, L_{3}$ are given by coordinates vanishing as follows: For any point

$$
p=(x, y, \tilde{y}, z, \xi, \eta, \tilde{\eta}, \zeta) \in T^{*}(X \times Y \times Y \times Z) \backslash 0,
$$

one has

$$
\begin{aligned}
D & =\{y-\tilde{y}=0, \eta-\tilde{\eta}=0\}, \\
L_{0} & =\{x-y=0, \xi-\eta=0, \tilde{y}-z=0, \tilde{\eta}-\zeta=0\}, \\
L_{1} & =\left\{x-y=0, \xi-\eta=0, \tilde{y}^{\prime}-z^{\prime}=0, \tilde{\eta}-\zeta=0, \tilde{\eta}^{\prime \prime}+\zeta^{\prime \prime}=0\right\}, \\
L_{2} & =\left\{x^{\prime}-y^{\prime}=0, \xi-\eta=0, \xi^{\prime \prime}+\eta^{\prime \prime}=0, \tilde{y}-z=0, \tilde{\eta}-\zeta=0\right\}, \\
L_{3} & =\left\{x^{\prime}-y^{\prime}=0, \xi-\eta=0, \xi^{\prime \prime}+\eta^{\prime \prime}=0, \tilde{y}^{\prime}-z^{\prime}=0, \tilde{\eta}-\zeta=0, \tilde{\eta}^{\prime \prime}+\zeta^{\prime \prime}=0\right\} .
\end{aligned}
$$

The intersections $D \cap L_{j}, j=0,1,2,3$ are determined by the following equations:

$$
\begin{aligned}
& D \cap L_{0}=\{x-y=0, \xi-\eta=0, y-z=0, \eta-\zeta=0\}, \\
& D \cap L_{1}=\left\{x-y=0, \xi^{\prime}-\eta^{\prime}=0, y^{\prime}-z^{\prime}=0, \eta^{\prime}-\zeta^{\prime}=0, \xi^{\prime \prime}=0, \eta^{\prime \prime}=0, \zeta^{\prime \prime}=0\right\}, \\
& D \cap L_{2}=\left\{x^{\prime}-y^{\prime}=0, \xi^{\prime}-\eta^{\prime}=0, y-z=0, \eta^{\prime}-\zeta^{\prime}=0, \xi^{\prime \prime}=0, \eta^{\prime \prime}=0, \zeta^{\prime \prime}=0\right\}, \\
& D \cap L_{3}=\left\{x^{\prime}-y^{\prime}=0, \xi^{\prime}-\eta^{\prime}=0, y^{\prime}-z^{\prime}=0, \eta^{\prime}-\zeta^{\prime}=0, \xi^{\prime \prime}=\eta^{\prime \prime}=\zeta^{\prime \prime}=0\right\} .
\end{aligned}
$$

This leads to the conclusion that the system $D \cap L_{j}, j=0,1,2,3$ is a CIS on $D$. Note that ( $\left.D, L_{1}, L_{2}\right)$ does not form an intersecting triple. We now verify the condition (2.3]), that 
is,

$$
T_{p} D \cap \sum T_{p} L_{j}=\sum T_{p}\left(D \cap L_{j}\right), \quad p \in \bigcap_{j=0}^{3} L_{j} \cap D .
$$

By formulas (2.5])-(2.54), one obtains

$$
\operatorname{dim}\left(\sum T_{p}\left(D \cap L_{j}\right)=\operatorname{dim} D-2\left(d+d^{\prime}\right) .\right.
$$

On the other hand,

$$
\begin{aligned}
\operatorname{dim}\left(T_{p} D \cap \sum T_{p} L_{j}\right) & =\operatorname{dim} D+\operatorname{dim} \sum L_{j}-\operatorname{dim}\left(T_{p} D+\sum T_{p} L_{j}\right) \\
& =\operatorname{dim} D+\operatorname{dim}\left(T_{p} D^{\sigma} \cap \bigcap T_{p} L_{j}\right)-\operatorname{dim}\left(\bigcap L_{j}\right) \\
& =\operatorname{dim} D+0-\operatorname{dim}\left(C_{0} \cap C_{1}\right) \times\left(\widetilde{C}_{0} \cap \widetilde{C}_{1}\right) \\
& =\operatorname{dim} D-2\left(2 d-d^{\prime \prime}\right)=\operatorname{dim} D-2\left(d+d^{\prime}\right)
\end{aligned}
$$

This implies (2.3]). Moreover,

$$
T_{p} L_{0}+T_{p} L_{3}=\sum_{j=0}^{3} T_{p} L_{j}, \quad \sum_{j=0,3}\left(T_{p}\left(D \cap L_{j}\right)=\sum_{j=0}^{3}\left(T_{p}\left(D \cap L_{j}\right)\right.\right.
$$

shows that $\left(D, L_{0}, L_{3}\right)$ forms a CIS. However, $\left(D, L_{1}, L_{2}\right)$ is not a CIS because $T_{p} D \cap\left(T_{p} L_{1}+T_{p} L_{2}\right)=T_{p}\left(D \cap\left(T_{p} L_{0}+T_{p} L_{3}\right)=\sum_{j=0}^{3} T_{p}\left(D \cap L_{j}\right) \supsetneq T_{p}\left(D \cap L_{1}\right)+T_{p}\left(D \cap L_{2}\right)\right.$.

Thus, in this example our assumptions are satisfied.

Similarly, the following example provides the second case when one obtains closedness of the classes of paired Lagrangian distributions.

Example 2.40. The canonical relations $C_{0}, C_{1}$ and $\widetilde{C}_{0}, \widetilde{C}_{1}$ satisfy the following properties

$$
\begin{aligned}
C_{j} \circ \widetilde{C}_{0} & =E_{0}, \quad j=0,1, \\
C_{j} \circ \widetilde{C}_{1} & =E_{1}, \quad j=0,1, \\
\left(C_{0} \cap C_{1}\right) \circ\left(\widetilde{C}_{0} \cap \widetilde{C}_{1}\right) & =E_{0} \cap E_{1} .
\end{aligned}
$$

For instance, let $X=Y=Z=\mathbb{R}^{d}$ with local coordinates $x=\left(x^{\prime}, x^{\prime \prime}, x^{\prime \prime \prime}\right), y=\left(y^{\prime}, y^{\prime \prime}, y^{\prime \prime \prime}\right)$ and $z=\left(z^{\prime}, z^{\prime \prime}, z^{\prime \prime \prime}\right)$. Then the canonical relations defined by

$$
\begin{aligned}
& C_{0}=N^{*}\{x-y=0\}^{\prime}, \quad C_{1}=N^{*}\left\{x^{\prime}-y^{\prime}=0, x^{\prime \prime \prime}-y^{\prime \prime \prime}=0\right\}^{\prime}, \\
& \widetilde{C}_{0}=N^{*}\left\{y^{\prime}-z^{\prime}=0\right\}^{\prime}, \widetilde{C}_{1}=N^{*}\left\{y^{\prime}-z^{\prime}=0, y^{\prime \prime \prime}-z^{\prime \prime \prime}=0\right\}^{\prime}, \\
& E_{0}=N^{*}\left\{y^{\prime}-z^{\prime}=0\right\}^{\prime}, \quad E_{1}=N^{*}\left\{y^{\prime}-z^{\prime}=0, y^{\prime \prime \prime}-z^{\prime \prime \prime}=0\right\}^{\prime} .
\end{aligned}
$$

satisfy our assumption. 


\subsection{Models for compositions}

\subsubsection{Models for strong compositions}

To summarize several examples where the weak composition holds, let $x=\left(x^{\prime}, x^{\prime \prime}, x^{\prime \prime \prime}, x^{i v}\right) \in$ $\mathbb{R}^{k_{1}} \times \mathbb{R}^{k_{2}} \times \mathbb{R}^{k_{3}} \times \mathbb{R}^{d_{X}-k_{1}-k_{2}-k_{3}}$ be splitting of coordinates in $X$ and similar notations for $y$ and $z$. Let us define the following canonical relations from $\left(T^{*} Y \backslash 0\right)$ to $\left(T^{*} X \backslash 0\right)$ :

$$
\begin{aligned}
& C_{0}=N^{*}\left\{x^{\prime}-y^{\prime}=0, x^{\prime \prime}-y^{\prime \prime}=0, x^{\prime \prime \prime}-y^{\prime \prime \prime}=0\right\}^{\prime}, \\
& C_{1}=N^{*}\left\{x^{\prime}-y^{\prime}=0, x^{\prime \prime \prime}-y^{\prime \prime \prime}=0\right\}^{\prime}, \\
& C_{2}=N^{*}\left\{x^{\prime \prime}-y^{\prime \prime}=0, x^{\prime \prime \prime}-y^{\prime \prime \prime}=0\right\}^{\prime}, \\
& C_{3}=N^{*}\left\{x^{\prime \prime \prime}-y^{\prime \prime \prime}=0\right\}^{\prime},
\end{aligned}
$$

and from $\left(T^{*} Z \backslash 0\right)$ to $\left(T^{*} Y \backslash 0\right)$ :

$$
\begin{aligned}
& D_{0}=N^{*}\left\{y^{\prime}-z^{\prime}=0, y^{\prime \prime}-z^{\prime \prime}=0, y^{\prime \prime \prime}-z^{\prime \prime \prime}=0\right\}^{\prime}, \\
& D_{1}=N^{*}\left\{y^{\prime}-z^{\prime}=0, y^{\prime \prime \prime}-z^{\prime \prime \prime}=0\right\}^{\prime}, \\
& D_{2}=N^{*}\left\{y^{\prime \prime}-z^{\prime \prime}=0, y^{\prime \prime \prime}-z^{\prime \prime \prime}=0\right\}^{\prime}, \\
& D_{3}=N^{*}\left\{y^{\prime \prime \prime}-z^{\prime \prime \prime}=0\right\}^{\prime} .
\end{aligned}
$$

Then we have the following weak compositions for properly supported operators:

\begin{tabular}{|l|l|l|l|l|}
\hline$A$ & $B$ & $A \circ B$ & Involved Lagrangian manifolds & note \\
\hline$I\left(C_{0}, C_{2}\right)$ & $I\left(D_{0}, D_{1}\right)$ & $I\left(E_{0}, E_{1}, E_{2}, E_{3}\right)$ & Four & Exam. 2.35 \\
$I\left(C_{0}, C_{1}\right)$ & $I\left(D_{2}, D_{3}\right)$ & $I\left(E_{1}, E_{2}, E_{3}\right)$ & $E_{0}, E_{2}$ and two times $E_{3}$ & Exam. 2.36 \\
$I\left(C_{0}, C_{1}\right)$ & $I\left(D_{0}, D_{1}\right)$ & $I\left(E_{0}, E_{1}\right)$ & $E_{0}$ and three times $E_{1}$ & Exam. 2.39 \\
$I\left(C_{0}, C_{2}\right)$ & $I\left(D_{2}, D_{3}\right)$ & $I\left(E_{2}, E_{3}\right)$ & 2 times $E_{1}$ and 2 times $E_{3}$ & Exam. 2.40 \\
$I\left(C_{1}, C_{3}\right)$ & $I\left(D_{2}, D_{3}\right)$ & $I\left(E_{3}\right)$ & 4 times $E_{3}$ & Exam. 2.37 \\
\hline
\end{tabular}

Table 2.1: Weak composition of paired Lagrangian distributions

where the canonical relations $E_{j}, j=0,1,2,3$ from $\left(T^{*} Z \backslash 0\right)$ to $\left(T^{*} X \backslash 0\right)$ are defined by:

$$
\begin{aligned}
& E_{0}=N^{*}\left\{x^{\prime}-z^{\prime}=0, x^{\prime \prime}-z^{\prime \prime}=0, x^{\prime \prime \prime}-z^{\prime \prime \prime}=0\right\}^{\prime}, \\
& E_{1}=N^{*}\left\{x^{\prime}-z^{\prime}=0, x^{\prime \prime \prime}-z^{\prime \prime \prime}=0\right\}^{\prime}, \\
& E_{2}=N^{*}\left\{x^{\prime \prime}-z^{\prime \prime}=0, x^{\prime \prime \prime}-z^{\prime \prime \prime}=0\right\}^{\prime}, \\
& E_{3}=N^{*}\left\{x^{\prime \prime \prime}-z^{\prime \prime \prime}=0\right\}^{\prime} .
\end{aligned}
$$


Now we shall prove some strong compositions corresponding to these cases.

Theorem 2.41. Let $C_{j}, D_{k}, E_{l}$ be canonical relations as above. Suppose that $A \in I^{m_{0}, m_{2}}\left(X, Y ; C_{0}, C_{2}\right)$ and $B \in I^{p_{0}, p_{1}}\left(Y, Z ; D_{0}, D_{1}\right)$ are two operators such that the projection into $X \times Z$ from $\left(\operatorname{supp} k_{A} \times \operatorname{supp} k_{B}\right) \cap\left(X \times \Delta_{Y} \times Z\right)$ is proper. Then $A \circ B \in I^{q_{0}, q_{1}, q_{2}, q_{3}}\left(X, Z ; E_{0}, E_{1}, E_{2}, E_{3}\right)$, where

$$
q_{0}=m_{0}+p_{0}+e / 2, q_{1}=m_{0}+p_{1}+e / 2, q_{2}=m_{2}+p_{0}+e / 2, q_{3}=m_{2}+p_{1}+e / 2
$$

with the excess $e=d_{Y}-\left(k_{1}+k_{2}+k_{3}\right)$.

Proof. Recall that $A \in I^{m_{0}, m_{2}}\left(X, Y ; C_{0}, C_{2}\right)$ means that its Schwartz kernel has the following representation

$$
k_{A}(x, y)=\int e^{i(x-y) \eta} a(x, y, \eta) d \eta
$$

where $a(x, y, \eta) \in S^{m_{0}^{\prime}, m_{2}^{\prime}}\left(X \times Y \times\left(\mathbb{R}^{k_{1}+k_{2}+k_{3}} \backslash 0\right)\right.$, i.e. for any compact subset $K \Subset X \times Y$ and multi-indices $\alpha, \beta$, there is a constant $C_{K, \alpha, \beta}$ such that

$$
\left|D_{x, y}^{\beta} D_{\eta}^{\alpha} a(x, y, \eta)\right| \leq C\langle\eta\rangle^{m_{2}^{\prime}-\left|\alpha^{\prime \prime}, \alpha^{\prime \prime \prime}\right|}\left\langle\eta^{\prime}\right\rangle^{m_{0}^{\prime}-m_{2}^{\prime}-\left|\alpha^{\prime}\right|},(x, y) \in K,
$$

with $m_{0}^{\prime}=m_{0}+\left(d_{X}+d_{Y}\right) / 4-\left(k_{1}+k_{2}+k_{3}\right) / 2, m_{2}^{\prime}=m_{2}+\left(d_{X}+d_{Y}\right) / 4-\left(k_{2}+k_{3}\right) / 2$. Similarly, $B \in I^{p_{0}, p_{1}}\left(Y, Z ; D_{0}, D_{1}\right)$ implies that

$$
k_{B}(y, z)=\int e^{i(y-z) \xi} b(y, z, \xi) d \xi
$$

where the function $b$ satisfies

$$
\left|D_{y, z}^{\beta} D_{\xi}^{\alpha} b(y, z, \xi)\right| \leq C\langle\xi\rangle^{p_{1}^{\prime}-\left|\alpha^{\prime}, \alpha^{\prime \prime \prime}\right|}\left\langle\xi^{\prime \prime}\right\rangle^{p_{0}^{\prime}-p_{1}^{\prime}-\left|\alpha^{\prime \prime}\right|},(y, z) \in K \Subset Y \times Z,
$$

with $p_{0}^{\prime}=p_{0}+\left(d_{Y}+d_{Z}\right) / 4-\left(k_{1}+k_{2}+k_{3}\right) / 2, p_{1}^{\prime}=p_{1}+\left(d_{Y}+d_{Z}\right) / 4-\left(k_{1}+k_{3}\right) / 2$. Then the composed operator $A \circ B$ has the Schwartz kernel

$$
k_{A \circ B}(x, z)=\int e^{i(x-y) \eta+i(y-z) \xi} a(x, y, \eta) b(x, z, \xi) d y d \eta d \xi .
$$

Without loss of generality, assume that $a(x, y, \eta)$ and $b(y, z, \xi)$ vanish when $|\eta| \leq 1$ and $|\xi| \leq 1$, respectively. If $|\xi| \leq|\eta| / 2$ or $|\eta| \leq|\xi| / 2$ in the support of $a(x, y, \eta) b(y, z, \xi)$, then we have $|\eta-\xi| \geq(|\eta|+|\xi|) / 2$ for all $(x, y, z, \eta, \xi) \in \operatorname{supp} a b$. Using integration by part in $y$, we conclude that this part contributes to $k_{A \circ B}(x, z)$ only smooth function. Hence, we make a further assumption that $|\eta| / 2 \leq|\eta| \leq 2|\xi|$ in the support of $a b$. This yields

$$
\left|D_{x, y, z}^{\gamma} D_{\eta}^{\beta} D_{\xi}^{\alpha}(a b)\right| \leq C\langle\xi, \eta\rangle^{m_{2}^{\prime}+p_{1}^{\prime}-\left|\alpha^{\prime}, \alpha^{\prime \prime \prime}\right|-\left|\beta^{\prime \prime}, \beta^{\prime \prime \prime}\right|}\left\langle\eta^{\prime}\right\rangle^{m_{0}^{\prime}-m_{2}^{\prime}-\left|\beta^{\prime}\right|}\left\langle\xi^{\prime \prime}\right\rangle^{p_{0}^{\prime}-p_{1}^{\prime}-\left|\alpha^{\prime \prime}\right|}
$$


for all $(x, y, z) \in K \Subset X \times Y \times Z$.

Because the variables $\xi^{\prime}, \eta^{\prime \prime}, \eta^{\prime \prime \prime}$ have the same behaviour as usual symbol of type $S_{1,0}$, using stationary phase method in $y^{\prime}, y^{\prime \prime}, y^{\prime \prime \prime}, \xi^{\prime}, \eta^{\prime \prime}, \eta^{\prime \prime \prime}$, we obtain:

$$
k_{A \circ B}(x, z)=\int e^{i\left(x^{\prime}-z^{\prime}\right) \eta^{\prime}+\left(x^{\prime \prime}-z^{\prime \prime}\right) \xi^{\prime \prime}+\left(x^{\prime \prime \prime}-z^{\prime \prime \prime}\right) \xi^{\prime \prime \prime}} c\left(x, z, \eta^{\prime}, \xi^{\prime \prime}, \xi^{\prime \prime \prime}, y^{(4)}\right) d \eta^{\prime} d \xi^{\prime \prime} d \xi^{\prime \prime \prime} d y^{(4)},
$$

where

$$
\begin{aligned}
c\left(x, z, \eta^{\prime}, \xi^{\prime \prime}, \xi^{\prime \prime \prime}, y^{(4)}\right) & =e^{i\left(y^{\prime}-z^{\prime}\right)\left(\eta^{\prime}-\xi^{\prime}\right)-i\left(x^{\prime \prime}-y^{\prime \prime}\right)\left(\eta^{\prime \prime}-\xi^{\prime \prime}\right)-i\left(x^{\prime \prime \prime}-y^{\prime \prime \prime}\right)\left(\eta^{\prime \prime \prime}-\xi^{\prime \prime \prime}\right)} a b d y^{\prime} d y^{\prime \prime} d y^{\prime \prime \prime} d \xi^{\prime} d \eta^{\prime \prime} d \eta^{\prime \prime \prime} \\
& =\left.e^{-i\left\langle D_{y^{\prime}}, D_{\xi^{\prime}}\right\rangle+i\left\langle D_{y^{\prime \prime}}, D_{\eta^{\prime \prime}}\right\rangle+i\left\langle D_{y^{\prime \prime \prime}}, D_{\eta^{\prime \prime \prime}}\right\rangle}(a(x, y, \eta) b(y, z, \xi))\right|_{V_{0}}, \\
& \left.\sim \sum_{j} \frac{1}{j !}\left(-i\left\langle D_{y^{\prime}}, D_{\xi^{\prime}}\right\rangle+i\left\langle D_{y^{\prime \prime}}, D_{\eta^{\prime \prime}}\right\rangle+i\left\langle D_{y^{\prime \prime \prime}}, D_{\eta^{\prime \prime \prime}}\right\rangle\right)^{j}(a b)\right|_{V_{0}},
\end{aligned}
$$

with $V_{0}=\left\{(x, y, z, \eta, \xi) \mid\left(y, \xi^{\prime}, \eta^{\prime \prime}, \eta^{\prime \prime \prime}\right)=\left(z^{\prime}, x^{\prime \prime}, x^{\prime \prime \prime}, \eta^{\prime}, \xi^{\prime \prime}, \xi^{\prime \prime \prime}\right)\right\}$. As a consequence, the symbol $a\left(x, z, \eta^{\prime}, \xi^{\prime \prime}, \xi^{\prime \prime \prime}, y^{(4)}\right)$ satisfies the following estimate:

$$
\left|D_{x, z, y^{(4)}}^{\beta} D_{\eta^{\prime}, \xi^{\prime \prime}, \xi^{\prime \prime \prime}}^{\alpha} c\left(x, z, \eta^{\prime}, \xi^{\prime \prime}, \xi^{\prime \prime \prime}, y^{(4)}\right)\right| \leq C\left\langle\eta^{\prime}, \xi^{\prime \prime}, \xi^{\prime \prime \prime}\right\rangle^{m_{2}^{\prime}+p_{1}^{\prime}-\left|\alpha^{\prime \prime \prime}\right|}\left\langle\eta^{\prime}\right\rangle^{m_{0}^{\prime}-m_{2}^{\prime}-\left|\alpha^{\prime}\right|}\left\langle\xi^{\prime \prime}\right\rangle^{p_{0}^{\prime}-p_{1}^{\prime}-\left|\alpha^{\prime \prime}\right|} .
$$

Therefore, $\tilde{c}\left(x, z, \eta^{\prime}, \xi^{\prime \prime}, \xi^{\prime \prime \prime}\right)=\int c\left(x, z, \eta^{\prime}, \xi^{\prime \prime}, \xi^{\prime \prime \prime}, y^{(4)}\right) d y^{(4)}$ also satisfies the same estimate above. This implies that $A \circ B \in I^{q}\left(X, Z ; E_{0}, E_{1}, E_{2}, E_{3}\right)$. By definition $A .26$, the order $q \in \mathbb{R}^{4}$ satisfies the following equations:

$$
\begin{aligned}
q_{1}^{\prime}+q_{2}^{\prime} & -q_{0}^{\prime}-q_{3}^{\prime}=0 \\
q_{0}^{\prime}-q_{2}^{\prime} & =m_{0}^{\prime}-m_{2}^{\prime}=m_{0}-m_{2}-k_{1} / 2 \\
q_{0}^{\prime}-q_{1}^{\prime} & =p_{0}^{\prime}-p_{1}^{\prime}=p_{0}-p_{1}-k_{2} / 2 \\
q_{3}^{\prime} & =m_{2}^{\prime}+p_{1}^{\prime}=m_{2}+\left(d_{X}+d_{Y}\right) / 4-\left(k_{2}+k_{3}\right) / 2+p_{1}+\left(d_{Y}+d_{Z}\right) / 4-\left(k_{1}+k_{3}\right) / 2 \\
q_{0}^{\prime} & =q_{0}+\left(d_{X}+d_{Z}\right) / 4-\left(k_{1}+k_{2}+k_{3}\right) / 2 \\
q_{1}^{\prime} & =q_{1}+\left(d_{X}+d_{Z}\right) / 4-\left(k_{1}+k_{3}\right) / 2 \\
q_{2}^{\prime} & =q_{2}+\left(d_{X}+d_{Z}\right) / 4-\left(k_{2}+k_{3}\right) / 2 \\
q_{3}^{\prime} & =q_{3}+\left(d_{X}+d_{Z}\right) / 4-k_{3} / 2
\end{aligned}
$$

Hence, $A \circ B \in I^{q}(X, Z ; E)$ with the correct order $q$ satisfying relation (2.58). The proof is completed.

More general, we have the following embedding maps

$$
\begin{aligned}
& I^{m_{0}, m_{2}}\left(C_{0}, C_{2}\right) \subset I^{m_{0}, m_{0}-k_{2} / 2, m_{2}, m_{2}-k_{2} / 2}\left(X, Y ; C_{0}, C_{1}, C_{2}, C_{3}\right), \\
& I^{p_{0}, p_{1}}\left(D_{0}, D_{1}\right) \subset I^{p_{0}, p_{1}, p_{0}-k_{1} / 2, p_{1}-k_{1} / 2}\left(Y, Z ; D_{0}, D_{1}, D_{2}, D_{3}\right) .
\end{aligned}
$$

Then the previous theorem is a special case of the following result. 
Proposition 2.42. Let $X, Y, Z, C_{i}, D_{j}, E_{k}$ be as above and $m, p \in \mathbb{R}^{4}$. Suppose that $A \in$ $I^{m}(X, Y ; C)$ and $B \in I^{p}(Y, Z ; D)$ are two properly operators, i.e., the Schwartz kernel $k_{A} \in$ $I^{m}\left(X \times Y, C^{\prime}\right), k_{B} \in I^{m}\left(Y \times Z, E^{\prime}\right)$. Then $A \circ B \in I^{m+p+e / 2}(X, Z ; E)$ where the excess $e \in \mathbb{R}^{4}$ is determined by $e_{0}=d_{Y}-\left(k_{1}+k_{2}+k_{3}\right), e_{1}=e_{0}+k_{2}, e_{2}=e_{0}+k_{1}, e_{3}=e_{0}+k_{1}+k_{2}$.

Lemma 2.43. If $a(x, y, \eta) \in S^{m}(X \times Y, C)$ and $b(y, z, \xi) \in S^{p}(Y \times Z, D)$ then the Gauss transform $\left.e^{i\left\langle D_{y}, D_{\eta}\right\rangle}(a(x, \eta) b(y, \xi))\right|_{(y, \eta)=(x, \xi)} \in S^{m+p}(X \times Z, E)$.

Proof. We will apply Hörmander's results on Gauss transforms [Theorem 18.4.10', Hör85] to conclude that $\left.e^{i\left\langle D_{y}, D_{\eta}\right\rangle} a(x, y, \eta) b(y, z, \xi)\right|_{(y, \eta)=(x, \xi)}$ belongs to $S(m+p, E)$. Let

$$
V=X \times Y \times Z \times \mathbb{R}^{2 N}
$$

with the coordinates $v=(x, y, z, \eta, \xi)$. Consider the following metric

$$
g_{v}(d v)=d x^{2}+d y^{2}+d z^{2}+\frac{\left|d \eta^{\prime}\right|^{2}}{1+\left|\eta^{\prime}\right|^{2}}+\frac{\left|d \eta^{\prime \prime}\right|^{2}}{1+|\eta|^{2}}+\frac{\left|d \xi^{\prime}\right|^{2}}{1+\left|\xi^{\prime}\right|^{2}}+\frac{\left|d \xi^{\prime \prime}\right|^{2}}{1+|\xi|^{2}}
$$

On $V$ we define weighted functions

$$
w(v)=\left\langle\eta^{\prime}, \eta^{\prime \prime}\right\rangle^{m_{0}}\left\langle\eta^{\prime}\right\rangle^{m_{1}}\left\langle\eta^{\prime \prime}\right\rangle^{m_{2}}\langle\eta\rangle^{m_{3}}\left\langle\xi^{\prime}, \xi^{\prime \prime}\right\rangle^{p_{0}}\left\langle\xi^{\prime}\right\rangle^{p_{1}}\left\langle\xi^{\prime \prime}\right\rangle^{p_{2}}\langle\xi\rangle^{p_{3}} \quad \forall m, p \in \mathbb{R}^{4} .
$$

The symmetric bilinear form on the dual $V^{\prime}$ of $V$ is given by

$$
\sigma(\hat{v}, \hat{\hat{v}})=\hat{\eta} \hat{\hat{y}}+\hat{y} \hat{\hat{\eta}}
$$

The dual metric of $g$ with respect to $\sigma$ is given by

$$
\begin{aligned}
g_{v}^{\sigma}(d v) & =\sup _{g_{v}(\sigma \hat{d} v)<1}\langle d v, \hat{d v}\rangle^{2} \\
& = \begin{cases}+\infty, & \text { if }|d x|^{2}+|d \xi|^{2}+|d z|^{2} \neq 0, \\
\left\langle\eta^{\prime}\right\rangle^{2}\left|d y^{\prime}\right|^{2}+\langle\eta\rangle^{2}\left|d y^{\prime \prime}\right|^{2}+|d \eta|^{2}, & \text { otherwise. }\end{cases}
\end{aligned}
$$

Let $V_{0}=\left.V\right|_{(y, \eta)=(x, \xi)}$ be a subspace of $V$. One has to verify the following properties:

- The metric $g$ is slowly varying in $V$. It is trivial by choosing $c<1 / 4$ and $C=2$.

- The weighted functions $w$ are $g$-continuous. It is also trivial with the same constants as above.

- The metric $g$ is $\sigma$-temperate and $w$ is $g, \sigma$-temperate with respect to $v$ uniformly in $V_{0}$. 
- The inequality $g_{v}(d v) \leq g_{v}^{\sigma}(d v)$. It is a consequence of (2.60) $)$.

It remains to check the third property. In order to obtain the temperateness of $g$, we verify

$$
\begin{aligned}
& g_{v}(d v) \leq g_{u}(d v)\left(1+g_{v}^{\sigma}(v-u)\right) \\
\Leftrightarrow & \sup _{d v \neq 0} \frac{g_{v}(d v)}{g_{u}(d v)} \leq\left(1+g_{v}^{\sigma}(v-u)\right),
\end{aligned}
$$

where $v=(x, y, z, \xi, \eta), u=(\tilde{x}, \tilde{y}, \tilde{z}, \tilde{\xi}, \tilde{\eta})$. Because $g$ is diagonal, one needs to prove that

$$
\max \left\{1, \frac{1+|\tilde{\xi}|^{2}}{1+|\xi|^{2}}, \frac{1+\left|\tilde{\xi}^{\prime}\right|^{2}}{1+\left|\xi^{\prime}\right|^{2}}, \frac{1+|\tilde{\eta}|^{2}}{1+|\eta|^{2}}, \frac{1+\left|\tilde{\eta}^{\prime}\right|^{2}}{1+\left|\eta^{\prime}\right|^{2}}\right\} \leq\left(1+g_{v}^{\sigma}(v-u)\right) .
$$

It follows from the inequality $1+g_{v}^{\sigma}(v-w) \geq\left(1+|\tilde{\eta}-\eta|^{2}+|\tilde{\xi}-\xi|^{2}\right)$. Similarly, one obtains

$$
\frac{w(v)}{w(u)} \leq\left(1+|\tilde{\xi}-\xi|^{2}+|\tilde{\eta}-\eta|^{2}\right)^{|m| / 2} \leq\left(1+g_{v}^{\sigma}(v-u)\right)^{|m| / 2},
$$

with $|m|=\sum_{j=0}^{3}\left|m_{j}\right|$. Hence, $w(v)$ is $\sigma, g$-temperate.

Theorem 2.44. Let $A \in I^{m_{0}, m_{2}}\left(C_{0}, C_{2}\right)$ and $B \in I^{p_{2}, p_{3}}\left(D_{2}, D_{3}\right)$ be two operators such that the projection from $\operatorname{supp} k_{A} \times \operatorname{supp} k_{B} \cap\left(X \times \Delta_{Y} \times Z\right)$ into $X \times Z$ is proper. Then $A \circ B \in I^{m_{2}+p_{2}+e / 2, m_{2}+p_{2}+e / 2}\left(E_{2}, E_{3}\right)$ with $e=d_{Y}-\left(k_{2}+k_{3}\right)$.

Proof. Since $A \in I^{m_{0}, m_{2}}\left(C_{0}, C_{2}\right)$, there exists a symbol $a(x, y, \xi)=a\left(x, y, \xi^{\prime}, \xi^{\prime \prime}, \xi^{\prime \prime \prime}\right)$ such that for each $K$ compact in $X \times Y$,

$$
\left|D_{x, y}^{\alpha} D_{\xi}^{\beta} a\left(x, y, \xi^{\prime}, \xi^{\prime \prime}, \xi^{\prime \prime \prime}\right)\right| \leq C_{K}\langle\xi\rangle^{m_{2}^{\prime}-\left|\beta^{\prime \prime}, \beta^{\prime \prime \prime}\right|}\left\langle\xi^{\prime}\right\rangle^{m_{0}^{\prime}-m_{2}^{\prime}-\left|\beta^{\prime}\right|},(x, y) \in K,
$$

with $m_{0}^{\prime}=m_{0}+\left(d_{X}+d_{Y}\right) / 4-\left(k_{1}+k_{2}+k_{3}\right) / 2, m_{2}^{\prime}=m_{2}+\left(d_{X}+d_{Y}\right) / 4-\left(k_{2}+k_{3}\right) / 2$ and for all $u \in C_{c}^{\infty}(Y)$ :

$$
\left.A u(x)=\int e^{i\left(\left(x^{\prime}-y^{\prime}\right) \xi^{\prime}+\left(x^{\prime \prime}-y^{\prime \prime}\right) \xi^{\prime \prime}+\left(x^{\prime \prime \prime}-y^{\prime \prime \prime}\right) \xi^{\prime \prime \prime}\right.}\right) a\left(x, y, \xi^{\prime}, \xi^{\prime \prime}, \xi^{\prime \prime \prime}\right) u(y) d y d \xi^{\prime} d \xi^{\prime \prime} d \xi^{\prime \prime \prime}
$$

Similarly, there exists $b(y, z, \eta)=b\left(y, z, \eta^{\prime \prime}, \eta^{\prime \prime \prime}\right)$ such that for all compact subset $K$ of $Y \times Z$,

$$
\left|D_{y, z}^{\alpha} D_{\eta^{\prime \prime}, \eta^{\prime \prime \prime}}^{\beta} b\left(y, z, \eta^{\prime \prime}, \eta^{\prime \prime \prime}\right)\right| \leq C\left\langle\eta^{\prime \prime}, \eta^{\prime \prime \prime}\right\rangle^{p_{3}^{\prime}-\left|\beta^{\prime \prime \prime}\right|}\left\langle\eta^{\prime \prime}\right\rangle^{p_{2}^{\prime}-p_{3}^{\prime}-\left|\beta^{\prime \prime}\right|},(y, z) \in K,
$$

where $p_{2}^{\prime}=p_{2}+\left(d_{Y}+d_{Z}\right) / 4-\left(k_{2}+k_{3}\right) / 2, p_{3}^{\prime}=p_{3}+\left(d_{Y}+d_{Z}\right) / 4-k_{3} / 2$ and for all $v \in C_{c}^{\infty}(Z)$,

$$
B v(y)=\int e^{i\left(\left(y^{\prime \prime}-z^{\prime \prime}\right) \eta^{\prime \prime}+\left(y^{\prime \prime \prime}-z^{\prime \prime \prime}\right) \eta^{\prime \prime \prime}\right)} b\left(y, z, \eta^{\prime \prime}, \eta^{\prime \prime \prime}\right) v(z) d z d \eta^{\prime \prime} d \eta^{\prime \prime \prime}
$$


This yields

$$
\begin{aligned}
k_{A \circ B}(x, z) & =\int e^{i\left(\left(x^{\prime}-y^{\prime}\right) \xi^{\prime}+\left(x^{\prime \prime}-y^{\prime \prime}\right) \xi^{\prime \prime}+\left(x^{\prime \prime \prime}-y^{\prime \prime \prime}\right) \xi^{\prime \prime \prime}\right)+i\left(\left(y^{\prime \prime}-z^{\prime \prime}\right) \eta^{\prime \prime}+\left(y^{\prime \prime \prime}-z^{\prime \prime \prime}\right) \eta^{\prime \prime \prime}\right)} a(x, y, \xi) b(y, z, \eta) d y d \xi d \eta, \\
& =e^{i\left(\left(x^{\prime \prime}-z^{\prime \prime}\right) \eta^{\prime \prime}+\left(x^{\prime \prime \prime}-z^{\prime \prime \prime}\right) \eta^{\prime \prime \prime}\right)} c\left(x, z, \eta^{\prime \prime}, \eta^{\prime \prime \prime}\right) d \eta^{\prime \prime} d \eta^{\prime \prime \prime},
\end{aligned}
$$

where $c\left(x, z, \eta^{\prime \prime}, \eta^{\prime \prime \prime}\right)=\int e^{i\left(\left(x^{\prime}-y^{\prime}\right) \xi^{\prime}+\left(x^{\prime \prime}-y^{\prime \prime}\right)\left(\xi^{\prime \prime}-\eta^{\prime \prime}\right)+\left(x^{\prime \prime \prime}-y^{\prime \prime \prime}\right)\left(\xi^{\prime \prime \prime}-\eta^{\prime \prime \prime}\right)\right)} a(x, y, \xi) b(y, z, \eta) d \xi d y$. Regroup $\hat{x}=\left(x^{\prime}, x^{\prime \prime}, x^{\prime \prime \prime}\right), \hat{y}=\left(y^{\prime}, y^{\prime \prime}, y^{\prime \prime \prime}\right)$ then the previous formula can be rewritten as

$$
\begin{aligned}
c\left(x, z, \eta^{\prime \prime}, \eta^{\prime \prime \prime}\right) & =\int \tilde{c}\left(x, z, \eta^{\prime \prime}, \eta^{\prime \prime \prime}, y^{(4)}\right) d y^{(4)} \\
\tilde{c}\left(x, z, \eta^{\prime \prime}, \eta^{\prime \prime \prime}, y^{(4)}\right) & =\left.e^{i\left\langle D_{\xi}, D_{\hat{y}}\right\rangle} a\left(x, \hat{y}, y^{(4)}, \xi\right) b\left(\hat{y}, y^{(4)}, z, \eta^{\prime \prime}, \eta^{\prime \prime \prime}\right)\right|_{\xi=\left(0, \eta^{\prime \prime}, \eta^{\prime \prime \prime}\right), \hat{y}=\hat{x}} .
\end{aligned}
$$

It follows from Hörmander results on Gauss transforms that $c \in S^{m_{2}^{\prime}+p_{2}^{\prime}, m_{2}^{\prime}+p_{3}^{\prime}}$ with extra parameter $y^{(4)}$. This implies that

$$
A \circ B \in I^{m_{2}+p_{2}+e / 2, m_{2}+p_{3}+e / 2}\left(E_{2}, E_{3}\right), \quad e=d_{Y}-\left(k_{2}+k_{3}\right) .
$$

The proof is completed.

Remark 2.45. It is not surprising that the order on $C_{0} \backslash C_{1}$ does not contribute to the composed operators. In fact, since $\left(C_{0} \backslash C_{2}\right) \circ\left(D_{2} \cup D_{3}\right)=\emptyset$, the composition takes place on $C_{2}$ only. However, this composition is still different from the composition of Fourier integral operator $A \in I\left(C_{2}\right)$ with the paired Lagrangian distribution $B \in I\left(D_{2}, D_{3}\right)$ since the intersection $C_{2} \cap C_{0}$ also contributes to the composite.

\subsubsection{Models for compositions without the CIS condition}

As we claim in the strong composition of FIO with PLD, if the CIS condition is violate, then the composed operator does not belong to the class of paired Lagrangian distribution associated with the natural cleanly intersecting pair of canonical relations. Now we introduce a model with this property:

Theorem 2.46. Let $X=\mathbb{R}^{d_{x}}, Y=\mathbb{R}^{d_{y}}$ and $Z=\mathbb{R}^{d_{z}}$. Consider canonical relations $C_{0}, C_{1}$ from $T^{*} Z \backslash 0$ to $T^{*} Y \backslash 0$ and $C$ from $T^{*} Y \backslash 0$ to $T^{*} X \backslash 0$ as follows:

$$
\begin{aligned}
C & =N^{*}\left\{y^{\prime \prime}-x^{\prime \prime}=0, y^{\prime \prime \prime}-x^{\prime \prime \prime}=0\right\}^{\prime}, \\
C_{0} & =N^{*}\left\{z^{\prime}-y^{\prime}=0, z^{\prime \prime}-y^{\prime \prime}=0, z^{\prime \prime \prime}-y^{\prime \prime \prime}=0\right\}^{\prime}, \\
C_{1} & =N^{*}\left\{z^{\prime}+y^{\prime}=0, z^{\prime \prime}-y^{\prime \prime}=0\right\}^{\prime}, \\
\widetilde{C}_{0} & =N^{*}\left\{x^{\prime \prime}-z^{\prime \prime}=0, x^{\prime \prime \prime}-z^{\prime \prime \prime}=0\right\}^{\prime}, \\
\widetilde{C}_{1} & =N^{*}\left\{x^{\prime \prime}-z^{\prime \prime}=0\right\}^{\prime}, \widetilde{\Sigma}=\left.\left(\widetilde{C}_{0} \cap \widetilde{C}_{1}\right)\right|_{z^{\prime}=0},
\end{aligned}
$$


where $x=\left(x^{\prime}, x^{\prime \prime}, x^{\prime \prime \prime}, x^{i v}\right), y=\left(y^{\prime}, y^{\prime \prime}, y^{\prime \prime \prime}, y^{i v}\right)$ and $z=\left(z^{\prime}, z^{\prime \prime}, z^{\prime \prime \prime}, z^{i v}\right)$ are coordinates of $X, Y$ and $Z$, respectively. Then

$$
C \circ C_{j}=\widetilde{C}_{j}, \quad j=0,1, \quad C \circ\left(C_{0} \cap C_{1}\right)=\widetilde{\Sigma} .
$$

Furthermore, for all properly supported operators $A \in I^{m}(C)$ and $B \in I^{m_{0}, m_{1}}\left(C_{0}, C_{1}\right)$, the composition

$$
A \circ B \in \sum_{j=0,1} I^{m_{j}+m+e / 2,\left(m_{0}+m_{1}\right) / 2+\left(2 k^{\prime}+k^{\prime \prime \prime}\right) / 4-m_{j}}\left(\widetilde{C}_{j}, \widetilde{\Sigma}\right)
$$

where $e=d_{y}-\left(k^{\prime}+k^{\prime \prime}+k^{\prime \prime \prime}\right)$ is the excess of the composition $C \circ C_{0}$.

With the same proof, one also gains the following result:

Theorem 2.47. Let $X=\mathbb{R}^{d_{x}}, Y=\mathbb{R}^{d_{y}}$ and $Z=\mathbb{R}^{d_{z}}$. Consider canonical relations $C_{0}, C_{1}$ from $T^{*} Y \backslash 0$ to $T^{*} X \backslash 0$ and $C$ from $T^{*} Z \backslash 0$ to $T^{*} Y \backslash 0$ as follows:

$$
\begin{aligned}
C_{0} & =N^{*}\left\{x^{\prime}-y^{\prime}=0, x^{\prime \prime}-y^{\prime \prime}=0, x^{\prime \prime \prime}-y^{\prime \prime \prime}=0\right\}^{\prime}, \\
C_{1} & =N^{*}\left\{x^{\prime}+y^{\prime}=0, x^{\prime \prime}-y^{\prime \prime}=0\right\}^{\prime}, \\
C & =N^{*}\left\{y^{\prime \prime}-z^{\prime \prime}=0, y^{\prime \prime \prime}-z^{\prime \prime \prime}=0\right\}^{\prime}, \\
\widetilde{C}_{0} & =N^{*}\left\{x^{\prime \prime}-z^{\prime \prime}=0, x^{\prime \prime \prime}-z^{\prime \prime \prime}=0\right\}^{\prime}, \\
\widetilde{C}_{1} & =N^{*}\left\{x^{\prime \prime}-z^{\prime \prime}=0\right\}^{\prime}, \widetilde{\Sigma}=\left.\left(\widetilde{C}_{0} \cap \widetilde{C}_{1}\right)\right|_{x^{\prime}=0},
\end{aligned}
$$

where $x=\left(x^{\prime}, x^{\prime \prime}, x^{\prime \prime \prime}, x^{i v}\right), y=\left(y^{\prime}, y^{\prime \prime}, y^{\prime \prime \prime}, y^{i v}\right)$ and $z=\left(z^{\prime}, z^{\prime \prime}, z^{\prime \prime \prime}, z^{i v}\right)$ are coordinates of $X, Y$ and $Z$, respectively. Then

$$
C_{j} \circ C=\widetilde{C}_{j}, \quad j=0,1, \quad\left(C_{0} \cap C_{1}\right) \circ C=\widetilde{\Sigma} .
$$

Furthermore, for all properly supported operators $A \in I^{m_{0}, m_{1}}\left(C_{0}, C_{1}\right)$ and $B \in I^{m}(C)$, the composition

$$
A \circ B \in \sum_{j=0,1} I^{m_{j}+m+e / 2,\left(m_{0}+m_{1}\right) / 2+\left(2 k^{\prime}+k^{\prime \prime \prime}\right) / 4-m_{j}}\left(\widetilde{C}_{j}, \widetilde{\Sigma}\right),
$$

where $e=d_{y}-\left(k^{\prime}+k^{\prime \prime}+k^{\prime \prime \prime}\right)$ is the excess of the composition $C_{0} \circ C$.

Proof. Obviously, $C_{0}$ and $C_{1}$ intersect cleanly at an isotropic submanifold $\Sigma$ of dimension $d_{x}+d_{y}-2 k^{\prime}-k^{\prime \prime \prime}$ and (2.62) holds. Decomposing paired Lagrangian distributions into sum of marked Lagrangian distributions [Jos99, Theorem 5.1], one has

$$
I^{m_{0}, m_{1}}\left(C_{0}, C_{1}\right)=I^{m_{0},\left(m_{1}-m_{0}\right) / 2+\left(2 k^{\prime}+k^{\prime \prime \prime}\right) / 4}\left(C_{0}, \Sigma\right)+I^{m_{1},\left(m_{0}-m_{1}\right) / 2+\left(2 k^{\prime}+k^{\prime \prime \prime}\right) / 4}\left(C_{1}, \Sigma\right) .
$$


Therefore, it remains to check

$$
I^{m_{j}, s}\left(C_{j}, \Sigma\right) \circ I^{m}(C) \subseteq I^{m_{j}+m+e / 2, s}\left(\widetilde{C}_{j}, \widetilde{\Sigma}\right), \quad j=0,1 .
$$

The proof is completed by using Lemma 2.48 below.

Lemma 2.48. If $A \in I^{m_{j}, s}\left(C_{j}, \Sigma\right)$ and $B \in I^{m}(C)$ are compactly supported operators, then

$$
A \circ B \in I^{m_{j}+m+e / 2, s}\left(\widetilde{C}_{j}, \widetilde{\Sigma}\right) .
$$

Proof. We shall prove the case $j=0$, while $j=1$ is proved similarly. Rewrite

$$
\hat{x}=\left(x^{\prime}, x^{\prime \prime}, x^{\prime \prime \prime}\right), \hat{y}=\left(y^{\prime}, y^{\prime \prime}, y^{\prime \prime \prime}\right) \text {. }
$$

By Proposition A.22, there exists a $C^{\infty}$ function $a\left(\hat{x}+\hat{y}, x^{i v}, y^{i v}, \xi^{\prime}, \xi^{\prime \prime}, \xi^{\prime \prime \prime}\right)$ such that

$$
K_{A}(x, y)=\int e^{i\left(\left(x^{\prime}-y^{\prime}\right) \xi^{\prime}+\left(x^{\prime \prime}-y^{\prime \prime}\right) \xi^{\prime \prime}+\left(x^{\prime \prime \prime}-y^{\prime \prime \prime}\right) \xi^{\prime \prime \prime}\right)} a\left(\hat{x}+\hat{y}, x^{i v}, y^{i v}, \xi^{\prime}, \xi^{\prime \prime}, \xi^{\prime \prime \prime}\right) d \xi^{\prime} d \xi^{\prime \prime} d \xi^{\prime \prime \prime},
$$

where the function $a$ satisfies the following estimate: for all $K \Subset X$, multi-indices $\alpha^{\prime}, \beta$, we have

$$
\left|D_{\hat{x}}^{\alpha} D_{\xi}^{\beta} D_{x^{i v}, y^{i v}}^{\gamma} a\left(\hat{x}, x^{i v}, y^{i v}, \xi\right)\right| \leq C_{K, \alpha, \beta}\left(\left|x^{\prime}\right|+\frac{\left|\xi^{\prime}, \xi^{\prime \prime \prime}\right|+\left\langle\xi^{\prime \prime}\right\rangle^{1 / 2}}{\langle\xi\rangle}\right)^{-2 s-\left|\alpha^{\prime}\right|-\left|\beta^{\prime}, \beta^{\prime \prime \prime}\right|}\langle\xi\rangle^{p^{\prime}-|\beta|},
$$

with $p^{\prime}=p+\left(d_{x}+d_{y}\right) / 4-\left(k^{\prime}+k^{\prime \prime}+k^{\prime \prime \prime}\right) / 2$.

Similarly, $B \in I^{m}(C)$ implies that

$$
K_{B}(y, z)=\int e^{i\left(\left(x^{\prime \prime}-y^{\prime \prime}\right) \eta^{\prime \prime}+\left(x^{\prime \prime \prime}-y^{\prime \prime \prime}\right) \eta^{\prime \prime \prime}\right)} b\left(y, z, \eta^{\prime \prime}, \eta^{\prime \prime \prime}\right) d \eta^{\prime \prime} d \eta^{\prime \prime \prime},
$$

where the function $b \in S^{m+\left(d_{y}+d_{z}\right) / 4-\left(k^{\prime \prime}+k^{\prime \prime \prime}\right) / 2}\left(Y \times Z \times \mathbb{R}^{k^{\prime \prime}+k^{\prime \prime \prime}}\right)$. The Schwartz kernel of the composition $A \circ B$ can be written as an oscillatory integral:

$$
\begin{aligned}
k_{A \circ B}(x, z) & =\int e^{i\left(\left(x^{\prime}-y^{\prime}\right) \xi^{\prime}+\left(x^{\prime \prime}-y^{\prime \prime}\right) \xi^{\prime \prime}+\left(x^{\prime \prime \prime}-y^{\prime \prime \prime}\right) \xi^{\prime \prime \prime}\right)+i\left(\left(y^{\prime \prime}-z^{\prime \prime}\right) \eta^{\prime \prime}+\left(y^{\prime \prime \prime}-z^{\prime \prime \prime}\right) \eta^{\prime \prime \prime}\right)} a(x, y, \xi) b(y, z, \eta) d y d \xi d \eta \\
& =\int e^{i\left(\left(x^{\prime \prime}-z^{\prime \prime}\right) \eta^{\prime \prime}+\left(x^{\prime \prime \prime}-z^{\prime \prime \prime}\right) \eta^{\prime \prime \prime}\right)} c\left(x, z, \eta^{\prime \prime}, \eta^{\prime \prime \prime}\right) d \eta^{\prime \prime} d \eta^{\prime \prime \prime},
\end{aligned}
$$

where $c\left(x, z, \eta^{\prime \prime}, \eta^{\prime \prime \prime}\right)=\int e^{i\left(\left(x^{\prime}-y^{\prime}\right) \xi^{\prime}+\left(x^{\prime \prime}-y^{\prime \prime}\right)\left(\xi^{\prime \prime}-\eta^{\prime \prime}\right)+\left(x^{\prime \prime \prime}-y^{\prime \prime \prime}\right)\left(\xi^{\prime \prime \prime}-\eta^{\prime \prime \prime}\right)\right)} a(x, y, \xi) b(y, z, \eta) d \xi d y$. The previous formula yields

$$
\begin{aligned}
c\left(x, z, \eta^{\prime \prime}, \eta^{\prime \prime \prime}\right) & =\int \tilde{c}\left(x, z, \eta^{\prime \prime}, \eta^{\prime \prime \prime}, y^{i v}\right) d y^{i v} \\
\tilde{c}\left(x, z, \eta^{\prime \prime}, \eta^{\prime \prime \prime}, y^{(4)}\right) & \left.=e^{i\left\langle D_{\xi}, D_{\hat{y}}\right.}\right\rangle\left._{a}\left(\hat{x}, \hat{y}, x^{i v}, y^{i v}, \xi\right) b\left(\hat{y}, y^{i v}, z, \eta^{\prime \prime}, \eta^{\prime \prime \prime}\right)\right|_{\xi=\left(0, \eta^{\prime \prime}, \eta^{\prime \prime \prime}\right), \hat{y}=\hat{x}}
\end{aligned}
$$


It follows from Hörmander results on Gauss transforms that $c(x, z, \eta)$ satisfies:

$$
\left|D_{x^{\prime}}^{\alpha} D_{\xi}^{\beta} D_{x^{i v}, z^{i v}}^{\gamma} c(x, z, \eta)\right| \leq C_{K, \alpha, \beta}\left(\left|x^{\prime}\right|+\frac{\left|\eta^{\prime \prime \prime}\right|+\left\langle\eta^{\prime \prime}\right\rangle^{1 / 2}}{\left\langle\eta^{\prime \prime}, \eta^{\prime \prime \prime}\right\rangle}\right)^{-2 s-|\alpha|-\left|\beta^{\prime \prime \prime}\right|}\left\langle\eta^{\prime \prime}, \eta^{\prime \prime \prime}\right\rangle^{p^{\prime}+m^{\prime}-\left|\beta^{\prime \prime}, \beta^{\prime \prime \prime}\right|} .
$$

But this exactly means that $a \in S^{p^{\prime}+m+e / 2, s}$. As a conclusion, one has $A \circ B \in I^{p+m+e / 2, s}\left(C_{0}, \widetilde{\Sigma}\right)$.

We are interested in the degenerate case when there is a canonical relation $\widetilde{C}$ from $T^{*} Z \backslash 0$ to $T^{*} X \backslash 0$ such that $\widetilde{C}=C_{j} \circ C, j=0,1$, and $\left(C_{0} \cap C_{1}\right) \circ C=\widetilde{\Sigma}$ is an isotropic submanifold, but $I\left(C_{0}, C_{1}\right) \circ I(C)=I(\widetilde{C}, \widetilde{\Sigma})$, the class of marked Lagrangian distributions. Let $y^{\prime \prime \prime}=\emptyset$ and rewrite $y^{\prime \prime \prime}$ instead of $y^{i v}$, one gets:

Corollary 2.49. Let $X=\mathbb{R}^{d_{x}}, Y=\mathbb{R}^{d_{y}}$ and $Z=\mathbb{R}^{d_{z}}$. Consider canonical relations $C_{0}, C_{1}$ from $T^{*} Y \backslash 0$ to $T^{*} X \backslash 0$ and $C$ from $T^{*} Z \backslash 0$ to $T^{*} Y \backslash 0$ as follows:

$$
\begin{aligned}
C_{0} & =N^{*}\left\{x^{\prime}-y^{\prime}=0, x^{\prime \prime}-y^{\prime \prime}=0\right\}^{\prime}, \\
C_{1} & =N^{*}\left\{x^{\prime}+y^{\prime}=0, x^{\prime \prime}-y^{\prime \prime}=0\right\}^{\prime}, \\
C & =N^{*}\left\{y^{\prime \prime}-z^{\prime \prime}=0\right\}^{\prime}, \\
\widetilde{C} & =N^{*}\left\{x^{\prime \prime}-z^{\prime \prime}=0\right\}^{\prime}, \quad \widetilde{\Sigma}=\left.\widetilde{C}\right|_{x^{\prime}=0},
\end{aligned}
$$

where $x=\left(x^{\prime}, x^{\prime \prime}, x^{\prime \prime \prime}\right), y=\left(y^{\prime}, y^{\prime \prime}, y^{\prime \prime \prime}\right)$ and $z=\left(z^{\prime}, z^{\prime \prime}, z^{\prime \prime \prime}\right)$ are coordinates of $X, Y$ and $Z$, respectively. Then

$$
C_{j} \circ C=\widetilde{C}, \quad j=0,1, \quad\left(C_{0} \cap C_{1}\right) \circ C=\widetilde{\Sigma} .
$$

Furthermore, for all properly supported operators $A \in I^{m_{0}, m_{1}}\left(C_{0}, C_{1}\right)$ and $B \in I^{m}(C)$, the composition

$$
A \circ B \in I^{\max \left\{m_{0}, m_{1}\right\}+m+e / 2,\left(m_{0}+m_{1}+k^{\prime}\right) / 2-\max \left\{m_{0}, m_{1}\right\}}(\widetilde{C}, \widetilde{\Sigma}),
$$

where $e=d_{y}-\#\left(y^{\prime}, y^{\prime \prime}\right)$ is the excess of the composition $C_{0} \circ C$.

We have seen that the violation of the CIS condition causes new singularities in the composition of FIOs and PLDs. An analogous phenomena takes place in the composition of PLDs. Indeed, let $C_{0}, C_{1}$ and $\widetilde{C}_{0}, \widetilde{C}_{1}$ be the following twisted conormal bundles to clean submanifolds:

$$
\begin{aligned}
& C_{0}=N^{*}\left\{x^{\prime}-y^{\prime}=0, x^{\prime \prime}-y^{\prime \prime}=0, x^{\prime \prime \prime}-y^{\prime \prime \prime}=0\right\}^{\prime}, \\
& C_{1}=N^{*}\left\{x^{\prime}+y^{\prime}=0, x^{\prime \prime}-y^{\prime \prime}=0, x^{\prime \prime \prime}-y^{\prime \prime \prime}=0\right\}^{\prime}, \\
& \widetilde{C}_{0}=N^{*}\left\{y^{\prime \prime}-z^{\prime \prime}=0, y^{\prime \prime \prime}-z^{\prime \prime \prime}=0\right\}^{\prime}, \\
& \widetilde{C}_{1}=N^{*}\left\{y^{\prime \prime \prime}-z^{\prime \prime \prime}=0\right\}^{\prime} .
\end{aligned}
$$


One can easily check that

$$
C_{j} \circ \widetilde{C}_{k}=E_{k}, \quad C_{j} \circ\left(\widetilde{C}_{0} \cap \widetilde{C}_{1}\right)=E_{0} \cap E_{1} \forall j, k=0,1 .
$$

However, one has

$$
\left(C_{0} \cap C_{1}\right) \circ \widetilde{C}_{j}=K_{j}, \quad\left(C_{0} \cap C_{1}\right) \circ\left(\widetilde{C}_{0} \cap \widetilde{C}_{1}\right)=K_{0} \cap K_{1} \subsetneq E_{0} \cap E_{1},
$$

where

$$
\begin{array}{lrl}
E_{0}=N^{*}\left\{x^{\prime \prime}-z^{\prime \prime}=0, x^{\prime \prime \prime}-z^{\prime \prime \prime}=0\right\}^{\prime}, & E_{1}=N^{*}\left\{x^{\prime \prime \prime}-z^{\prime \prime \prime}=0\right\}^{\prime}, \\
K_{j}=\left.E_{j}\right|_{x^{\prime}=0}, & j=0,1, & K_{0} \cap K_{1}=\left.E_{0} \cap E_{1}\right|_{x^{\prime}=0},
\end{array}
$$

with $x=\left(x^{\prime}, x^{\prime \prime}, x^{\prime \prime \prime}, x^{(4)}\right), y=\left(y^{\prime}, y^{\prime \prime}, y^{\prime \prime \prime}, y^{(4)}\right)$ and $z=\left(z^{\prime}, z^{\prime \prime}, z^{\prime \prime \prime}, z^{(4)}\right)$ are coordinates on $X, Y$ and $Z$, respectively.

Actually, we show that the resulting operators stay in a more general class of distributions associated with such Lagrangian manifolds with some isotropic marking on them, which combine paired Lagrangian distributions and marked Lagrangian distributions in the sense of Melrose [Mel87]. More precisely, we have

Definition 2.50. Let $\left(\Lambda_{0}, \Lambda_{1}\right)$ be a cleanly intersecting pair of closed conic Lagrangian manifolds of $T^{*} X \backslash 0$ and $M_{j}$ be a conic submanifold of $\Lambda_{j}$ for $j=0,1$. The set $\mathcal{M}\left(\Lambda_{0}, \Lambda_{1}, M_{0}, M_{1}\right)$ consists of all function $p \in \mathcal{M}\left(\Lambda_{0}, \Lambda_{1}\right)$ whose Hamiltonian vector field is tangent to $M_{0}, M_{1}$. The class of distributions on $X$ which is determined by the defining class of symbol $\mathcal{M}\left(\Lambda_{0}, \Lambda_{1}, M_{0}, M_{1}\right)$ will be denoted by $I\left(X ; \Lambda_{0}, \Lambda_{1}, M_{0}, M_{1}\right)$.

In our case, $\left(E_{0}^{\prime}, E_{1}^{\prime}\right)$ is a paired of closed conic Lagrangian manifolds of $T^{*}(X \times Z) \backslash 0$ and $K_{j}^{\prime}$ is conic submanifolds of $E_{j}^{\prime}$. This leads to the following observation:

Definition 2.51. Let $I\left(E_{0}, E_{1}, K_{0}, K_{1}\right)$ denote the class of operators associated with the system $\left(E_{0}, E_{1}, K_{0}, K_{1}\right)$ which consists of all operators $A: C_{c}^{\infty}(Z) \rightarrow \mathcal{D}^{\prime}(X)$ such that its Schwartz kernel $k_{A} \in I\left(X \times Z ; E_{0}^{\prime}, E_{1}^{\prime}, K_{0}^{\prime}, K_{1}^{\prime}\right)$.

Remark 2.52. Because of the identification ': $T^{*} X \times T^{*} Z \rightarrow T^{*}(X \times Z)$, we identify a defining class of symbols with its pull-back by the symplectomorphism above.

Theorem 2.53. Let $A \in I\left(X \times Y ; C_{0}, C_{1}\right)$ and $B \in I\left(Y \times Z ; \widetilde{C}_{0}, \widetilde{C}_{1}\right)$ be properly supported operators. Then $A \circ B$ is a properly supported operator belonging to the class

$$
I\left(X \times Z, E_{0}, E_{1}, K_{0}, K_{1}\right) .
$$


Proof. The left hand side is determined by the following defining class of symbols

$$
\mathcal{M}\left(E_{0}, E_{1}, K_{0}, K_{1}\right):=\left\{p(x, z, \xi, \zeta) \in \mathcal{M}\left(E_{0}, E_{1}\right) \mid \forall \gamma \in K_{j}^{\prime}, H_{p}(\gamma) \in T_{\gamma}\left(K_{j}^{\prime}\right), j=0,1\right\},
$$

where $H_{p}$ is the Hamiltonian vector field corresponding to $p$. Because $K_{j}^{\prime}, j=0,1$ are isotropic conic submanifolds, this class of symbols is closed under Poisson bracket. We have to show that the composite belongs to $I\left(E_{0}, E_{1}, K_{0}, K_{1}\right)$, the distributional space corresponding to the class $\mathcal{M}\left(E_{0}, E_{1}, K_{0}, K_{1}\right)$. Because $E_{0}, E_{1}$ are given by 2.77 , the space $\mathcal{M}\left(E_{0}, E_{1}\right)$ is spanned by the following functions

$$
\begin{aligned}
& \xi^{\prime}, \\
& \zeta^{\prime},\left(x^{\prime \prime}-z^{\prime \prime}\right)\left(\xi^{\prime \prime}-\zeta^{\prime \prime}\right),\left(x^{\prime \prime \prime}-z^{\prime \prime \prime}\right)|\xi, \zeta|, \xi^{\prime \prime \prime}-\zeta^{\prime \prime \prime}, \xi^{(4)}, \zeta^{(4)} .
\end{aligned}
$$

Evidently, for any $p \in \mathcal{M}\left(E_{0}, E_{1}\right)$, the vector $H_{p}$ tangents to $E_{j}$. Hence, $H_{p}$ is tangent to $K_{j}$ if and only if $H_{p}\left(d x^{\prime}\right)=0$. This yields $H_{x^{\prime}}(d p)=\partial_{\xi^{\prime}} p=0$ at $K_{j}$. Thus $\mathcal{M}\left(E_{0}, E_{1}, K_{0}, K_{1}\right)$ has the following generators

$$
\begin{aligned}
& x^{\prime} \xi^{\prime}, \frac{\xi^{\prime} \xi^{\prime}}{|\xi, \zeta|}, \frac{\xi^{\prime}\left(\xi^{\prime \prime}-\zeta^{\prime \prime}\right)}{|\xi, \zeta|},\left(x^{\prime \prime}-z^{\prime \prime}\right) \xi^{\prime} \\
& \zeta^{\prime},\left(x^{\prime \prime}-z^{\prime \prime}\right)\left(\xi^{\prime \prime}-\zeta^{\prime \prime}\right),\left(x^{\prime \prime \prime}-z^{\prime \prime \prime}\right)|\xi, \zeta|, \xi^{\prime \prime \prime}-\zeta^{\prime \prime \prime}, \xi^{(4)}, \zeta^{(4)}
\end{aligned}
$$

We need to verify that any function $f(x, z, \xi, \zeta) \in \mathcal{M}$, considered as a function on

$$
D=\left(T^{*} X \backslash 0\right) \times \Delta_{T^{*} Y \backslash 0} \times\left(T^{*} Z \backslash 0\right),
$$

is the restriction to $D$ of some defining function $\tilde{f} \in \mathcal{M}\left(C_{j} \times \tilde{C}_{k}, j, k=0,1\right)$, the set of all first order symbols on $X \times Y \times Y \times Z$ vanishing on $C_{j} \times \widetilde{C}_{k}$ for all $j, k=0,1$.

On the other hand, the set $\mathcal{M}\left(C_{j} \times \tilde{C}_{k}, j, k=0,1\right)$ is determined by the following generators

$$
\begin{array}{r}
\left(x^{\prime}-y^{\prime}\right)\left(x^{\prime}+y^{\prime}\right)|\xi, \eta, \tilde{\eta}, \zeta|,\left(x^{\prime}+y^{\prime}\right)\left(\xi^{\prime}-\eta^{\prime}\right),\left(x^{\prime}-y^{\prime}\right)\left(\xi^{\prime}+\eta^{\prime}\right), \frac{\left(\xi^{\prime}+\eta^{\prime}\right)\left(\xi^{\prime}-\eta^{\prime}\right)}{|\xi, \eta, \tilde{\eta}, \zeta|}, \\
\left(x^{\prime \prime}-y^{\prime \prime}\right)|\xi, \eta, \tilde{\eta}, \zeta|,\left(x^{\prime \prime \prime}-y^{\prime \prime \prime}\right)|\xi, \eta, \tilde{\eta}, \zeta|, \xi^{\prime \prime}-\eta^{\prime \prime}, \xi^{\prime \prime \prime}-\eta^{\prime \prime \prime}, \xi^{(4)}, \eta^{(4)}, \\
\tilde{\eta}^{\prime}, \zeta^{\prime},\left(\tilde{y}^{\prime \prime \prime}-z^{\prime \prime \prime}\right)|\xi, \eta, \tilde{\eta}, \zeta|, \tilde{\eta}^{\prime \prime \prime}-\zeta^{\prime \prime \prime}, \tilde{\eta}^{(4)}, \zeta^{(4)},\left(\tilde{y}^{\prime \prime}-z^{\prime \prime}\right)\left(\tilde{\eta}^{\prime \prime}-\zeta^{\prime \prime}\right) .
\end{array}
$$

Restricting to $y=\tilde{y}, \eta=\tilde{\eta}$, one obtains the following ideal

$$
\begin{array}{r}
\left\langle\left(x^{\prime}-y^{\prime}\right)\left(x^{\prime}+y^{\prime}\right)|\xi, \eta, \zeta|,\left(x^{\prime} \pm y^{\prime}\right) \xi^{\prime}, \frac{\left(\xi^{\prime}-\eta^{\prime}\right)\left(\xi^{\prime}+\eta^{\prime}\right)}{|\xi, \eta, \zeta|},\right. \\
\left(x^{\prime \prime}-y^{\prime \prime}\right)|\xi, \eta, \zeta|,\left(x^{\prime \prime \prime}-y^{\prime \prime \prime}\right)|\xi, \eta, \zeta|, \xi^{\prime \prime}-\eta^{\prime \prime}, \xi^{\prime \prime \prime}-\eta^{\prime \prime \prime}, \xi^{(4)}, \eta^{(4)}, \\
\left.\eta^{\prime}, \zeta^{\prime},\left(y^{\prime \prime \prime}-z^{\prime \prime \prime}\right)|\xi, \eta, \zeta|, \eta^{\prime \prime \prime}-\zeta^{\prime \prime \prime},\left(y^{\prime \prime}-z^{\prime \prime}\right)\left(\eta^{\prime \prime}-\zeta^{\prime \prime}\right), \zeta^{(4)}\right\rangle .
\end{array}
$$


Therefore, the conclusion easily follows. For instance, we have

$$
\begin{aligned}
x^{\prime} \xi^{\prime} & =\frac{\left(x^{\prime}-y^{\prime}\right) \xi^{\prime}+\left(x^{\prime}+y^{\prime}\right) \xi^{\prime}}{2}, \\
\left(x^{\prime \prime}-z^{\prime \prime}\right) \xi^{\prime} & =\left(x^{\prime \prime}-y^{\prime \prime}\right) \xi^{\prime}+\left(y^{\prime \prime}-z^{\prime \prime}\right) \xi^{\prime}, \\
\left(x^{\prime \prime \prime}-z^{\prime \prime \prime}\right)|\xi, \zeta| & =\left(x^{\prime \prime \prime}-y^{\prime \prime \prime}\right)|\xi, \zeta|+\left(y^{\prime \prime \prime}-z^{\prime \prime \prime}\right)|\xi, \zeta|, \\
\left(x^{\prime \prime}-z^{\prime \prime}\right)\left(\xi^{\prime \prime}-\zeta^{\prime \prime}\right) & =\left(x^{\prime \prime}-z^{\prime \prime}\right)\left(\xi^{\prime \prime}-\eta^{\prime \prime}\right)+\left(x^{\prime \prime}-y^{\prime \prime}\right)\left(\eta^{\prime \prime}-\zeta^{\prime \prime}\right)+\left(y^{\prime \prime}-z^{\prime \prime}\right)\left(\eta^{\prime \prime}-\zeta^{\prime \prime}\right), \\
\xi^{\prime \prime \prime}-\zeta^{\prime \prime \prime} & =\left(\xi^{\prime \prime}-\eta^{\prime \prime}\right)+\left(\eta^{\prime \prime}-\zeta^{\prime \prime}\right), \\
\frac{\xi^{2}}{|\xi, \zeta|} & =\frac{\left(\xi^{\prime}+\eta^{\prime}\right)\left(\xi^{\prime}-\eta^{\prime}\right)}{|\xi, \zeta|}+\frac{\eta^{2}}{|\xi, \zeta|} .
\end{aligned}
$$

The proof is completed. 


\section{A Appendix}

\section{A.1 Distribution theory}

Let $X$ be an open subset of $\mathbb{R}^{d}$ and $\mathcal{D}^{\prime}(X)$ be the set of all distributions in $X$.

Definition A.1. Let $u \in \mathcal{D}^{\prime}(X)$ and $\left(x_{0}, \xi_{0}\right) \in X \times\left(\mathbb{R}^{d} \backslash 0\right)$. We say that $\left(x_{0}, \xi_{0}\right) \notin \operatorname{WF}(u)$ if there exist a neighbourhood $V$ of $x_{0}$ and a conic neighbourhood $W$ of $\xi_{0}$ in $\left(\mathbb{R}^{d} \backslash 0\right)$ such that for some function $\chi \in C_{c}^{\infty}(V), \chi\left(x_{0}\right) \neq 0$, the Fourier transform $\widehat{\chi u}$ rapidly decreases in $W$ in the sense that for all $N \in \mathbb{R}$, there is a constant $C>0$ such that

$$
|\widehat{\chi u}(\xi)| \leq C\langle\xi\rangle^{-N}, \quad \xi \in W
$$

Note that $\operatorname{WF}(u)$ is a closed conic subset of $X \times\left(\mathbb{R}^{d} \backslash 0\right)$ whose projection into $X$ is equal to the singular support of $u$. In general, any closed conic subset of $X \times\left(\mathbb{R}^{d} \backslash 0\right)$ can be wave front set of some distribution $u \in \mathcal{D}^{\prime}(X)$.

With the help of pseudo-differential operators, one can use the following result as definition of wave front set of sectional distributions on manifold. The proof can be found in [Hör85, Chapter 18].

Proposition A.2. Let $\Psi^{m}(X)$ be the set of all classical properly supported pseudo-differential operators $P$ of order $m$ on $X$ with principal symbol $\sigma(P)$. For any $m \in \mathbb{R}$ and $u \in \mathcal{D}^{\prime}(X)$ we have

$$
\mathrm{WF}(u)=\bigcap_{\substack{A \in \Psi^{m}(X) \\ P u \in C^{\infty}(X)}} \text { Char } P,
$$

where Char $P=\left\{\left(x_{0}, \xi_{0}\right) \in T^{*} X \backslash 0 \mid \sigma(P)\left(x_{0}, \xi_{0}\right)=0\right\}$.

The following results shall tell us how wave front sets behave under fundamental operations such as tensor product, push-forward and pull-back. 
Theorem A.3 (Tensor product). Let $X$ be an open subset in $\mathbb{R}^{d_{1}}$ and $Y$ be an open subset in $\mathbb{R}^{d_{2}}$. For all $u \in \mathcal{D}^{\prime}(X), v \in \mathcal{D}^{\prime}(Y), u \otimes v$ defines a distribution in $D^{\prime}(X \times Y)$ and moreover,

$$
\mathrm{WF}(u \otimes v) \subseteq \mathrm{WF}(u) \times \mathrm{WF}(v) \bigcup(\operatorname{supp} u \times 0) \times \mathrm{WF}(v) \bigcup \mathrm{WF}(u) \times(\operatorname{supp} v \times 0) .
$$

where one identifies $X \times Y \times \mathbb{R}^{d_{1}+d_{2}}$ with $X \times \mathbb{R}^{d_{1}} \times Y \times \mathbb{R}^{d_{2}}$.

Theorem A.4 (pull-back). Suppose that $f: X \rightarrow Y$ is a smooth map and $f^{*}: C_{c}^{\infty}(Y) \rightarrow$ $C^{\infty}(X)$ is the pull-back operator defined by $f^{*} u=u \circ f$. Set

$$
N_{f}=\left\{(f(x), \eta) \mid x \in X, \eta \in \mathbb{R}^{d_{2}} \backslash 0,{ }^{t} d f(x) \eta=0\right\} .
$$

Then $f^{*}$ extends uniquely to an operator

$$
f^{*}:\left\{u \in \mathcal{D}^{\prime}(V) \mid \mathrm{WF}(u) \cap N_{f}=\emptyset\right\} \rightarrow \mathcal{D}^{\prime}(X) .
$$

Moreover,

$$
\mathrm{WF}\left(f^{*} u\right) \subseteq\left\{\left(x,{ }^{t} d f(x) \eta\right) \mid(f(x), \eta) \in \mathrm{WF}(u)\right\}
$$

Theorem A.5 (push-forward). Suppose that $Y, Z$ are smooth manifolds endowed positive densities $v, \theta$. Let

$$
\pi_{*}: C_{c}^{\infty}(Y \times Z) \rightarrow C_{c}^{\infty}(Y)
$$

be the operator defined by

$$
\pi_{*}(u)(y)=\int_{Z} u(y, z) v, \quad u \in C_{c}^{\infty}(Y \times Z) .
$$

Then $\pi_{*}$ can be extended to an operator

$$
\pi_{*}:\left\{u \in \mathcal{D}^{\prime}(Y \times Z) \mid \pi: \operatorname{supp} u \rightarrow Y \text { proper }\right\} \rightarrow \mathcal{D}^{\prime}(Y)
$$

Moreover,

$$
\mathrm{WF}\left(\pi_{*} u\right) \subseteq\left\{(y, \eta) \in T^{*} Y \backslash 0 \mid \exists z \in Z,(y, z, \eta, 0) \in \mathrm{WF}(u)\right\} .
$$

In composition theorem, it is convenient to work with wave front relation:

Definition A.6. Let $A: C_{c}^{\infty}(Y) \rightarrow \mathcal{D}^{\prime}(X)$ be a linear continuous operator. Wave front relation $\mathrm{WF}^{\prime}(A) \subseteq T^{*} X \times T^{*} Y$ of $A$ is defined by the following relation

$$
\mathrm{WF}^{\prime}(A)=\left\{(x, \xi ; y, \eta) \mid(x, y, \xi,-\eta) \in \mathrm{WF}\left(k_{A}\right)\right\},
$$

where $k_{A} \in \mathcal{D}^{\prime}(X \times Y)$ denotes Schwartz kernel of $A$. 
In order to formulate composition, one introduces the following sets:

$$
\begin{aligned}
\mathrm{WF}_{X}(A) & =\left\{(x, \xi) \in T^{*} X \mid \exists y \in Y,(x, \xi ; y, 0) \in \mathrm{WF}^{\prime}(A)\right\}, \\
\mathrm{WF}_{Y}^{\prime}(A) & =\left\{(y, \eta) \in T^{*} Y \mid \exists x \in X,(x, 0 ; y, \eta) \in \mathrm{WF}^{\prime}(A)\right\} \\
\Delta_{Y} & =\left\{\left(y_{1}, y_{2}\right) \in Y \times Y \mid y_{1}=y_{2}\right\} .
\end{aligned}
$$

Definition A.7. A continuous map $f: X \rightarrow Y$ is proper if for any compact subset $K \Subset Y$, its inverse image $f^{-1}(K)$ is a compact subset in $X$.

We recall a result about action of an operator in distributional class:

Theorem A.8. Let $A: C_{c}^{\infty}(Y) \rightarrow \mathcal{D}^{\prime}(X)$. For all $u \in \mathcal{D}^{\prime}(Y)$ such that $\operatorname{WF}_{Y}^{\prime}(A) \cap \mathrm{WF}(u)=\emptyset$ and the projection $\left(\operatorname{supp} k_{A} \times \operatorname{supp} u\right) \cap\left(X \times \Delta_{Y}\right) \rightarrow X$ is proper, Au is well-defined as an element in $\mathcal{D}^{\prime}(X)$. Moreover,

$$
\mathrm{WF}(u) \subseteq \mathrm{WF}^{\prime}(A) \circ \mathrm{WF}(u) \bigcup \mathrm{WF}_{X}(A)
$$

Remark A.9. For most interesting operator, $\mathrm{WF}_{X}(A)=\emptyset, \mathrm{WF}_{Y}^{\prime}(A)=\emptyset$, one can interpret the formula above as the propagation of singularities under the action of operator $A$.

It is a special case (and also an equivalent form) of the following abstract composition:

Theorem A.10 (composition). Let $X, Y, Z$ be smooth manifolds and $A: C_{c}^{\infty}(Y) \rightarrow \mathcal{D}^{\prime}(X)$ and $B: C_{c}^{\infty}(Z) \rightarrow \mathcal{D}^{\prime}(Y)$ be linear continuous operators. Suppose that $\mathrm{WF}_{Y}^{\prime}(A) \cap \mathrm{WF}_{Y}(B)=$ $\emptyset$, and the projection

$$
\pi:\left(\operatorname{supp} k_{A} \times \operatorname{supp} k_{B}\right) \bigcap\left(X \times \Delta_{Y} \times Z\right) \rightarrow X \times Z
$$

is proper. Then $A \circ B$ is well-defined as an operator from $C_{c}^{\infty}(Z) \rightarrow \mathcal{D}^{\prime}(X)$. Furthermore,

$$
\mathrm{WF}^{\prime}(A \circ B) \subseteq \mathrm{WF}^{\prime}(A) \circ \mathrm{WF}^{\prime}(B) \bigcup \mathrm{WF}_{X}(A) \times 0_{Z} \bigcup 0_{X} \times \mathrm{WF}_{Z}^{\prime}(B),
$$

where $0_{X}=X \times\{0\}, 0_{Z}=Z \times\{0\}$ as the zero sections in the cotangent bundles $T^{*} X$ and $T^{*} Z$, respectively.

Proof. Let $\Delta: X \times Y \times Z \rightarrow X \times Y \times Y \times Z$ be the diagonal map. The kernel of operator $A \circ B$ can be rewritten as

$$
k_{A \circ B}(x, z)=\pi_{*} \Delta^{*}\left(k_{A} \otimes k_{B}\right) .
$$


Note that $P:=\pi_{*} \Delta^{*}$ is a FIO associated with canonical relation $\Gamma$ from $T^{*}(X \times Y \times Y \times Z) \backslash 0$ to $T^{*}(X \times Z) \backslash 0$ which is defined by $\Gamma^{\prime}=N^{*}\left\{\Delta_{X \times Y \times Z}\right\}$. Hence, the operator $P$ has the following properties:

$$
\mathrm{WF}^{\prime}(P) \subseteq \Gamma, \mathrm{WF}_{X \times Y \times Y \times Z}^{\prime}(P)=0_{X} \times N_{Y \times Y}^{*}\left(\Delta_{Y}\right) \times 0_{Z}, \mathrm{WF}_{X \times Z}(P)=\emptyset .
$$

This implies that $(x, y, \tilde{y}, z, \xi, \eta, \tilde{\eta}, \zeta) \in \mathrm{WF}\left(k_{A} \times k_{B}\right) \cap \mathrm{WF}_{X \times Y \times Y \times Z}^{\prime}(P)$ if and only if

$$
\xi=0, \zeta=0, y=\tilde{y}, \eta=-\tilde{\eta}, \quad(x, y, y, z, 0, \eta,-\eta, 0) \in \mathrm{WF}\left(k_{A} \times k_{B}\right) .
$$

It is equivalent to $(y,-\eta) \in \mathrm{WF}_{Y}^{\prime}(A) \cap \mathrm{WF}_{Y}(B)=\emptyset$. Therefore, the composition theorem follows by applying action of $P$ on $k_{A} \otimes k_{B}$.

\section{A.2 Gauss transforms}

In this section we recall basic definitions and main results on Gauss transforms. For more details, see [Hör85, Section 18.4]. From now on $V$ denotes a finite dimensional vector space and $g_{x}, x \in V$ is a family of Riemannian metric in $V$.

Definition A.11. The metric $g$ is said to be slowly varying if there exist positive constant $c, C$ such that

$$
g_{x}(y)<c \Longrightarrow g_{x+y}(t) \leq C g_{x}(t) .
$$

Definition A.12. If $g$ is slowly varying, then a positive real-valued function $m: V \rightarrow \mathbb{R}$ is called $g$ continuous if there exist positive constants $c, C$ such that

$$
g_{x}(y)<c \Longrightarrow m(x) / C \leq m(x+y) \leq C m(x) .
$$

Denote by $S(m, g)$ the set of all $u \in C^{\infty}(V)$ such that

$$
\sup |u|_{k}^{g}(x) / m(x):=\sup _{t_{j} \in V}\left|u^{(k)}\left(x ; t_{1}, \ldots, t_{k}\right)\right| / \prod_{1}^{k} g_{x}\left(t_{j}\right)<+\infty,
$$

for all $k \in \mathbb{N}$.

$S(m, g)$ forms a Fréchet space with the topology defined by the semi-norms $p_{k}, k \in \mathbb{N}$ given by (A.15). Now let $A$ be a quadratic form on $V^{\prime}$, the dual space of $V$. The operator $e^{i A(D)}$ on $\mathcal{S}^{\prime}(V)$ is given by

$$
e^{i A(D)} u(x)=\mathcal{F}^{-1}\left(e^{i A(\xi)} \hat{u}(\xi)\right)
$$


Definition A.13. If $g$ is a positive definite quadratic form on $V$ and $A$ is a quadratic form on $V^{\prime}$, then the dual form of $g$ with respect to $A$ is a quadratic form on $V$ given as follows

$$
g^{A}(x)=\sup _{g(A \xi) \leq 1}\langle x, \xi\rangle^{2}
$$

Definition A.14. The Riemannian metric $g$ (and the positive function $m$ ) in $V$ is called $A$ temperate (resp. $A, g$ temperate) with respect to $x \in V$ if $g$ is slowly varying ( $m$ is $g$ continuous) and there exist constants $C$ and $N$ such that $y, t \in V$

$$
\begin{aligned}
& g_{y}(t) \leq C g_{x}(t)\left(1+g_{y}^{A}(x-y)\right)^{N} \\
& m(y) \leq C m(x)\left(1+g_{y}^{A}(x-y)\right)^{N}
\end{aligned}
$$

Definition A.15. A continuous linear form on $S(m, g)$ is called weakly continuous if the restriction to a bounded subset is continuous in the $C^{\infty}$ topology.

We shall use the following result on Gauss transforms

Theorem A.16 (Hörmander). The map $C_{c}^{\infty} \ni u \mapsto \exp i A(D) u(x) \in \mathbb{C}$ has a unique extension to a weakly continuous linear form on $(m, g)$ for every $x$ such that $g$ is A temperate, $g_{x} \leq g_{x}^{A}$, and $m$ is $A, g$ temperate with respect to $x$. We have

$$
|\exp i A(D) u(x)| \leq m(x)\|u\|
$$

Furthermore, if these conditions are fulfilled uniformly for all $x$ in a linear subspace $V_{0}$ of $V$, then the map

$$
\left.S(m, g) \ni u \mapsto \exp i A(D) u\right|_{V_{0}}
$$

is weakly continuous with values in the space $\left.S(m, g)\right|_{V_{0}}$ of symbol in $V_{0}$ corresponding to the restrictions of $m$ and of $g$.

\section{A.3 Marked Lagrangian distributions}

In this section we recall the definition of marked Lagrangian distributions, paired Lagrangian distributions and prove an oscillatory integral representation of these distributions in a model which is convenient for examining composition theorem. Let $X=\mathbb{R}^{d}$ and $x=\left(x^{\prime}, x^{\prime \prime}\right)$ be coordinates in $X$. First, we define

$$
\Lambda_{0}=N^{*}\{x=0\}, \quad K_{0}=\left.\Lambda\right|_{\xi^{\prime}=0} .
$$


Definition A.17 (Melrose). The space of marked Lagrangian distributions of order $(m, s) \in$ $\mathbb{R}^{2}$ associated with $\left(\Lambda_{0}, K_{0}\right)$ (denote by $I^{m, s}\left(\Lambda_{0}, K_{0}\right)$ ) consists of all distributions $u$ such that

$$
u(x)-(2 \pi)^{-n / 2} \int e^{i x \xi} a(\xi) d \xi \in C^{\infty}(X),
$$

for some $a \in S^{m^{\prime}, s}\left(\Lambda_{0}, K_{0}\right), m^{\prime}=m-d / 4$, i.e., $a \in C^{\infty}\left(\mathbb{R}^{d}\right)$ satisfies

$$
\left|D_{\xi}^{\alpha} a(\xi)\right| \leq C\left(\frac{\left(1+\left|\xi^{\prime}\right|^{4}+\left|\xi^{\prime \prime}\right|^{2}\right)^{1 / 4}}{\langle\xi\rangle}\right)^{-2 s-\left|\alpha^{\prime}\right|}\langle\xi\rangle^{m^{\prime}-|\alpha|} .
$$

Remark A.18. We can extend the classes of symbols depending on $x$ such that the oscillatory integral above defines the same classes of distributions. Namely, if $a(x, \xi) \in$ $C^{\infty}\left(X \times \mathbb{R}^{d}\right)$ such that for all multi-indices $\alpha, \beta$, there exists a constant $C$ satisfying

$$
\left|D_{\xi}^{\alpha} D_{x}^{\beta} a(x, \xi)\right| \leq C\left(\frac{\left(1+\left|\xi^{\prime}\right|^{4}+\left|\xi^{\prime \prime}\right|^{2}\right)^{1 / 4}}{\langle\xi\rangle}\right)^{-2 s-\left|\alpha^{\prime}\right|}\langle\xi\rangle^{m^{\prime}-|\alpha|} .
$$

Then $u(x)=\int e^{i x \xi} a(x, \xi) d \xi$ belongs to $I^{m, s}\left(\Lambda_{0}, K_{0}\right)$.

In fact, using Taylor expansion near $x=0$, it is evident that every symbol $a(x, \xi)$ can be reduced to a symbol independent of $x$. Taking $\tilde{a}(\xi)=\left.e^{-i\left\langle D_{x}, D_{\xi}\right\rangle} a(x, \xi)\right|_{x=0} \in S^{m^{\prime}, s}\left(\Lambda_{0}, K_{0}\right)$, then $u(x)-\int e^{i x \xi} \tilde{a}(\xi) d \xi \in C^{\infty}(X)$. Therefore, it is enough to consider the symbol classes which depend only on $\xi$.

Definition A.19 (Melrose, Joshi). Let $\Lambda$ be a conic embedded Lagrangian manifold of $T^{*} X \backslash 0$ and $K$ be a conic embedded submanifold of $\Lambda$. Denote $I^{m, s}(\Lambda, K)$, the space of marked Lagrangian distributions of order $(m, s)$ associated with $(\Lambda, K)$. It consists of distributions $u$ with $\mathrm{WF}(u) \subseteq \Lambda$, and for each $\lambda \in \Lambda$, there is a properly supported Fourier integral operator $F$ such that $F u \in I^{m, s}\left(\Lambda_{0}, K_{0}\right)$, where $F$ is of order zero, elliptic at $\lambda$ associated with a canonical transformation mapping $(\Lambda, K)$ into $\left(\Lambda_{0}, K_{0}\right)$.

Joshi also proved oscillatory integral representation of marked Lagrangian distributions in the following models, when splitting variable $x=\left(x^{\prime}, x^{\prime \prime}, x^{\prime \prime \prime}\right)$ :

$$
\begin{array}{ll}
\Lambda_{1}=N^{*}\left\{x^{\prime \prime \prime}=0\right\}, & K_{1}=\left.\Lambda_{1}\right|_{x^{\prime}=0}, \\
\Lambda_{2}=N^{*}\left\{x^{\prime \prime}=0, x^{\prime \prime \prime}=0\right\}, & K_{2}=\left.\Lambda_{2}\right|_{\xi^{\prime \prime}=0} .
\end{array}
$$

Proposition A.20 (Joshi). For all $(m, s) \in \mathbb{R}^{2}$, a distribution $u \in I^{m, s}\left(\Lambda_{1}, K_{1}\right)$ if and only if $u=u_{1}+u_{2}$, where $u_{1} \in C^{\infty}$ and

$$
u_{2}(x)=\int e^{i x^{\prime \prime \prime} \xi^{\prime \prime \prime}} a\left(x^{\prime}, x^{\prime \prime}, \xi^{\prime \prime \prime}\right) d \xi^{\prime \prime \prime}
$$


with $a \in S^{m^{\prime}, s}\left(\Lambda_{1}, K_{1}\right)$, the space of all smooth function $a\left(x^{\prime}, x^{\prime \prime}, \xi^{\prime \prime \prime}\right)$ satisfying

$$
\left|D_{x^{\prime}, x^{\prime \prime}}^{\alpha} D_{\xi^{\prime \prime \prime}}^{\beta} a\left(x^{\prime}, x^{\prime \prime}, \xi^{\prime \prime \prime}\right)\right| \leq C_{K, \alpha, \beta}\left(\left|x^{\prime}\right|+\left\langle\xi^{\prime \prime \prime}\right\rangle^{-1 / 2}\right)^{-2 s-\left|\alpha^{\prime}\right|}\left\langle\xi^{\prime \prime \prime}\right\rangle^{m^{\prime}-|\beta|}
$$

for $\left(x^{\prime}, x^{\prime \prime}\right) \in K$ compact, $m^{\prime}=m+d / 4-k^{\prime \prime \prime} / 2$, all multi-indices $\alpha, \beta$.

For more details, see [ [0s99, Section 4].

Now we shall investigate a generalized model with splitting $x=\left(x^{\prime}, x^{\prime \prime}, x^{\prime \prime \prime}, x^{i v}\right)$,

$$
\Lambda_{3}=N^{*}\left\{x^{\prime \prime}=0, x^{\prime \prime \prime}=0\right\}, \quad K_{3}=\left.\Lambda_{3}\right|_{x^{\prime}=0, \xi^{\prime \prime}=0} .
$$

Note that when $x^{i v}, \xi^{\prime \prime}$ or $x^{\prime}, x^{i v}$ are degenerate, one obtains the two models above.

Definition A.21. The space $S^{m, s}\left(\Lambda_{3}, K_{3}\right)$ consists of all $a\left(x, \xi^{\prime \prime}, \xi^{\prime \prime \prime}\right) \in C^{\infty}$ such that for all $x \in K \Subset X$,

$$
\left|D_{x}^{\beta} D_{\xi}^{\alpha} a(x, \xi)\right| \leq C_{K, \alpha, \beta}\left(\left|x^{\prime}\right|+\frac{\left(1+\left|\xi^{\prime \prime}\right|^{4}+\left|\xi^{\prime \prime \prime}\right|^{2}\right)^{1 / 4}}{\left\langle\xi^{\prime \prime}, \xi^{\prime \prime \prime}\right\rangle}\right)^{-2 s-\left|\alpha^{\prime}\right|-\left|\beta^{\prime \prime}\right|}\left\langle\xi^{\prime \prime}, \xi^{\prime \prime \prime}\right\rangle^{m^{\prime}-\left|\beta^{\prime \prime}, \beta^{\prime \prime \prime}\right|}
$$

Proposition A.22. A distribution $u \in I^{m, s}\left(\Lambda_{3}, K_{3}\right)$ if and only if there exists a function

$$
a\left(x, \xi^{\prime \prime}, \xi^{\prime \prime \prime}\right) \in S^{m^{\prime}, s}\left(\Lambda_{3}, K_{3}\right), m^{\prime}=m+d / 4-\left(k^{\prime \prime}+k^{\prime \prime \prime}\right) / 2
$$

such that

$$
u(x)-\int a\left(x, \xi^{\prime \prime}, \xi^{\prime \prime \prime}\right) e^{i\left(x^{\prime \prime} \xi^{\prime \prime}+x^{\prime \prime \prime} \xi^{\prime \prime \prime}\right)} d \xi^{\prime \prime} d \xi^{\prime \prime \prime} \in C^{\infty}(X) .
$$

Proof. We use the standard argument in the proof of model $\left(\Lambda_{1}, K_{1}\right)$, see [ [0s99, Theorem 4.2]. Consider a canonical transformation $\chi$ mapping $\left(\Lambda_{3}, K_{3}\right)$ into the model $\left(\Lambda_{0}, K_{0}\right)$. The map $\chi$ is defined away from $\left|\xi^{\prime \prime \prime}\right|=0$ as follows:

$\chi:(x, \xi) \rightarrow\left(\frac{-x^{\prime}}{\left|\xi^{\prime \prime}, \xi^{\prime \prime \prime}\right|},\left(x^{\prime \prime}, x^{\prime \prime \prime}\right)+\frac{x^{\prime} \xi^{\prime}+x^{i v} \xi^{i v}}{\left|\xi^{\prime \prime}, \xi^{\prime \prime \prime}\right|^{2}}\left(\xi^{\prime \prime}, \xi^{\prime \prime \prime}\right), \frac{-x^{i v}}{\left|\xi^{\prime \prime}, \xi^{\prime \prime \prime}\right|}, x^{\prime}\left|\xi^{\prime \prime}, \xi^{\prime \prime \prime}\right|,\left(\xi^{\prime \prime}, \xi^{\prime \prime \prime}\right), x^{i v}\left|\xi^{\prime \prime}, \xi^{\prime \prime \prime}\right|\right)$.

Rewrite $x_{I}=\left(x^{\prime}, x^{i v}\right), x_{I I}=\left(x^{\prime \prime}, x^{\prime \prime \prime}\right)$, then the phase function associated with the canonical transformation above is given by

$$
\Psi\left(y, x, \eta, \xi_{I}\right)=y_{I} \eta_{I}+\left(y_{I I}-x_{I I}\right) \eta_{I I}-x_{I} \xi_{I}+\frac{\eta_{I} \xi_{I}}{\left|\eta_{I I}\right|},
$$

Note that $\chi\left(\Lambda_{3}\right) \subseteq \Lambda_{0}:=N^{*}\{x=0\},\left.\quad \chi\left(K_{3}\right) \subseteq \Lambda_{0}\right|_{\xi^{\prime}=0, \xi^{\prime \prime}=0}$. Let $P$ be a properly supported Fourier integral operator of order zero such that

$$
P v(y)=\int e^{i \Psi\left(y, x, \eta, \xi_{I}\right)} p\left(y, x, \eta, \xi_{I}\right) v(x) d x d \eta d \xi_{I},
$$


where $\left.p \in S^{-k_{I} / 2}\left(Y \times \mathbb{R}^{(} k_{I}+d\right)\right)$, is elliptic near $K_{3}$ and smooth outside $K_{3}$. Assume that $u(x)=\int e^{i x_{I I} \xi_{I I}} a\left(x_{I}, \xi_{I I}\right) d \xi_{I I}$, for some $a \in S^{m^{\prime}, s}\left(\Lambda_{3}, K_{3}\right)$ with compact support in $x_{I}$. We shall show that $P u \in I^{m, s}\left(\Lambda_{0}, K_{0}\right)$. Indeed, we have

$$
\begin{aligned}
P u(y) & =\int e^{i y \eta}\left(\int e^{i\left(-x_{I} \xi_{I}+x_{I I}\left(\xi_{I I}-\eta_{I I}\right)+\xi_{I} \eta_{I} / \eta_{I I} \mid\right)} p\left(y, \eta, \xi_{I}\right) a\left(x, \xi_{I I}\right) d x d \xi\right) d \eta \\
& =\int e^{i y \eta} b(y, \eta) d \eta,
\end{aligned}
$$

where $b(y, \eta)=\int e^{i\left(-x_{I} \xi_{I}+x_{I I}\left(\xi_{I I}-\eta_{I I}\right)+\xi_{I} \eta_{I} /\left|\eta_{I I}\right|\right)} p\left(y, \eta, \xi_{I}\right) a\left(x, \xi_{I I}\right) d x d \xi$. First of all, by applying stationary phase method in variables $x_{I I}, \xi_{I I}$, it reduces to prove that

$$
\begin{aligned}
b(y, \eta) & =\int e^{-i\left(x_{I}-\frac{\eta_{I}}{\left|\eta_{I I}\right|}\right) \xi_{I}} p\left(x, \eta, \xi_{I}\right) a\left(x_{I}, \eta_{I I}\right) d x_{I} d \xi_{I}, \\
& =\int e^{i x_{I} \xi_{I}} p\left(y, \eta,-\xi_{I}\right) a\left(x_{I}+\frac{\eta_{I}}{\eta_{I I}}, \eta_{I I}\right) d x_{I} d \xi_{I} \\
& =\left.e^{i\left\langle D_{x_{I}}, D_{\xi_{I}}\right\rangle} p\left(y, \eta, \xi_{I}\right) a\left(x_{I}, \eta_{I I}\right)\right|_{x_{I}=\eta_{I} /\left|\eta_{I I}\right| \xi_{I}=0}
\end{aligned}
$$

belongs to $S^{m-d / 4, s}\left(\Lambda_{0} \times \mathbb{R}_{y}, K_{0}\right)$. It is easy to check that if $a\left(x_{I}, \eta_{I I}\right) \in S^{m, s}\left(\Lambda_{3}, K_{3}\right)$, then $\tilde{a}(\eta)=a\left(\eta_{I} /\left|\eta_{I I}\right|, \eta_{I I}\right) \in S^{m, s}\left(\Lambda_{0}, K_{0}\right)$. In fact, since $a\left(x_{I}, \eta_{I I}\right)$ has compact support in $x_{I}$, in the support of $a\left(\eta_{I} /\left|\eta_{I I}\right|, \eta_{I I}\right)$, one has

$$
\left|\eta_{I I}\right| \leq\left|\eta_{I}, \eta_{I I}\right| \leq C\left|\eta_{I I}\right|
$$

Replacing $x^{\prime}$ by $\eta^{\prime} /\left|\eta^{\prime \prime}, \eta^{\prime \prime \prime}\right|$ implies

$$
\left|x^{\prime}\right|^{2}+\frac{\left\langle\eta^{\prime \prime}\right\rangle^{2}+\left\langle\eta^{\prime \prime \prime}\right\rangle}{\left\langle\eta^{\prime \prime}, \eta^{\prime \prime \prime}\right\rangle^{2}} \simeq \frac{\left\langle\eta^{\prime}, \eta^{\prime \prime}\right\rangle^{2}+\left\langle\eta^{\prime \prime \prime}\right\rangle}{\left\langle\eta^{\prime \prime}, \eta^{\prime \prime \prime}\right\rangle^{2}} \simeq \frac{\left\langle\eta^{\prime}, \eta^{\prime \prime}\right\rangle^{2}+\left\langle\eta^{\prime \prime \prime}, \eta^{i v}\right\rangle}{\left\langle\eta_{I}, \eta_{I I}\right\rangle^{2}}
$$

Therefore, one obtains

$$
\begin{aligned}
|\tilde{a}(\eta)| & =\left|a\left(\frac{\eta_{I}}{\left|\eta_{I I}\right|}, \eta_{I I}\right)\right| \\
& \leq C\left(\mid \frac{\eta^{\prime}}{\left|\eta^{\prime \prime}, \eta^{\prime \prime \prime}\right|}+\frac{\left(1+\left|\eta^{\prime \prime}\right|^{4}+\left|\eta^{\prime \prime \prime}\right|^{2}\right)^{1 / 4}}{\left\langle\eta^{\prime \prime}, \eta^{\prime \prime \prime}\right\rangle}\right)^{-2 s}\left\langle\eta^{\prime \prime}, \eta^{\prime \prime \prime}\right\rangle^{m} \\
& \leq C\left(\frac{\left\langle\eta^{\prime}, \eta^{\prime \prime}\right\rangle+\left\langle\eta^{\prime \prime \prime}, \eta^{i v}\right\rangle^{1 / 2}}{\left\langle\eta_{I}, \eta_{I I}\right\rangle}\right)^{-2 s}\left\langle\eta_{I}, \eta_{I I}\right\rangle^{m} .
\end{aligned}
$$

The derivatives of $\tilde{a}\left(\eta_{I}, \eta_{I I}\right)$ are estimated in the same way.

This yields

$$
b(y, \eta)=\left.\sum_{|\alpha|}\left\langle i D_{x_{I}}, D_{\xi_{I}}\right\rangle^{\alpha} p\left(y, \eta, \xi_{I}\right) a\left(x_{I}, \eta_{I I}\right)\right|_{x_{I}=\eta_{I} /\left|\eta_{I I}\right| \xi_{I}=0} \in S^{m-d / 4}\left(\Lambda_{0}, K_{0}\right) .
$$

Hence $P u \in I^{m, s}\left(\Lambda_{0}, K_{0}\right)$. The first part was proved completed. The second part is proved similarly. 


\section{A.4 The four-Lagrangian distributions}

In this section we shall define a class of distributions corresponding to a given system of four Lagrangian manifolds $L_{j}, j=0,1,2,3$ of $T^{*} X \backslash 0$.

Definition A.23. Let denote by $\mathcal{M}\left(L_{0}, L_{1}, L_{2}, L_{3}\right)$ the set of the principal symbols of first order, properly supported pseudo-differential operators on $X$ which characterize on $L_{j}, j=$ $0,1,2,3$.

It is evident that $\mathcal{M}\left(L_{0}, L_{1}, L_{2}, L_{3}\right)$ is closed under Poisson bracket. Thus, the set $\mathcal{M}\left(L_{0}, L_{1}, L_{2}, L_{3}\right)$ is a defining class of symbols on $X$.

Definition A.24. The space of distributions on $X$ associated with the system of Lagrangian manifolds $L=\left\{L_{j}, j=0,1,2,3\right\}, I(X ; L)$, is determined by the class $\mathcal{M}(L)$.

We shall prove an oscillatory integral representation of distributions associated with the following four conormal Lagrangian submanifolds in $\mathbb{R}^{d} \times\left(\mathbb{R}^{d} \backslash 0\right)$ :

$$
\begin{array}{ll}
L_{0}=N^{*} S_{0}, & S_{0}=\left\{x^{\prime}=0, x^{\prime \prime}=0, x^{\prime \prime \prime}=0\right\}, \\
L_{1}=N^{*} S_{1}, & S_{1}=\left\{x^{\prime}=0, x^{\prime \prime \prime}=0\right\}, \\
L_{2}=N^{*} S_{2}, & S_{2}=\left\{x^{\prime \prime}=0, x^{\prime \prime \prime}=0\right\}, \\
L_{3}=N^{*} S_{3}, & S_{3}=\left\{x^{\prime \prime \prime}=0\right\},
\end{array}
$$

where $x^{\prime}=\left(x_{1}, \ldots, x_{d^{\prime}}\right), x^{\prime \prime}=\left(x_{d^{\prime}+1}, \ldots, d_{d^{\prime}+d^{\prime \prime}}\right), x^{\prime \prime \prime}=\left(x_{d^{\prime}+d^{\prime \prime}+1}, \ldots, x_{d^{\prime}+d^{\prime \prime}+d^{\prime \prime \prime}}\right)$ and $x^{(4)}=$ $\left(x_{d^{\prime}+d^{\prime \prime}+d^{\prime \prime \prime}+1}, \ldots x_{d}\right), x=\left(x^{\prime}, x^{\prime \prime}, x^{\prime \prime \prime}, x^{(4)}\right) \in \mathbb{R}^{d}$. For $m=\left(m_{0}, m_{1}, m_{2}, m_{3}\right) \in \mathbb{R}^{4}$, the basic weighted functions are given by

$$
\begin{aligned}
m(\xi) & =\left\langle\xi^{\prime}, \xi^{\prime \prime}\right\rangle^{m_{0}}\left\langle\xi^{\prime}\right\rangle^{m_{1}}\left\langle\xi^{\prime \prime}\right\rangle^{m_{2}}\langle\xi\rangle^{m_{3}}, \\
w_{m}(\xi) & =\left\langle\xi^{\prime}, \xi^{\prime \prime}\right\rangle^{m_{1}+m_{2}-m_{0}-m_{3}}\left\langle\xi^{\prime}\right\rangle^{m_{0}-m_{2}}\left\langle\xi^{\prime \prime}\right\rangle^{m_{0}-m_{1}}\langle\xi\rangle^{m_{3}}
\end{aligned}
$$

From this definition, we obtain the following properties of the weighted functions

$$
\begin{aligned}
\langle\xi\rangle^{m_{3}-\sum_{j=0}^{2} \max \left\{m_{j}, 0\right\}} \leq m(\xi) \leq\langle\xi\rangle^{m_{3}+\sum_{j=0}^{2} \max \left\{m_{j}, 0\right\}}, & & m \in \mathbb{R}^{4}, & \\
\langle\xi\rangle^{\min m_{j}} & \leq w_{m}(\xi) \leq\langle\xi\rangle^{\max m_{j}}, & & m \in \mathbb{R}^{4} \\
\left(w_{m}(\xi) w_{p}(\xi)\right. & =w_{m+p}(\xi), & & m, p \in \mathbb{R}^{4}, \\
w_{m}(\xi+\eta) & \leq C w_{m}(\eta) \cdot\langle\xi\rangle^{4 \max \left|m_{j}\right|}, & & m \in \mathbb{R}^{4} \\
w_{m}(\xi) & \leq w_{p}(\xi), & & m, p \in \mathbb{R}^{4}, m_{j} \leq p_{j}, j=0,1,2,3 .
\end{aligned}
$$


Definition A.25. The space $S(L, m)$ is the set of all smooth functions $a(x, \xi)$ such that for all multi-indices $\alpha, \beta$ and compact subset $K \Subset \mathbb{R}^{d}$, there is a positive constant $C=C_{K, \alpha, \beta}$

$$
\left|D_{x}^{\beta} D_{\xi}^{\alpha} a(x, \xi)\right| \leq C w_{m}(\xi)\left\langle\xi^{\prime}\right\rangle^{-\left|\alpha^{\prime}\right|}\left\langle\xi^{\prime \prime}\right\rangle^{-\left|\alpha^{\prime \prime}\right|}\langle\xi\rangle^{-\left|\alpha^{\prime \prime \prime}\right|}, x \in K
$$

One easily checks that

$$
\begin{aligned}
S(L, m) \cdot S\left(L, m^{\prime}\right) \ni(a, b) & \mapsto a b \in S\left(L, m+m^{\prime}\right) \\
D_{x}^{\beta} D_{\xi}^{\alpha}: S(L, m) & \rightarrow S\left(L, m-e_{\alpha}\right), \quad e_{\alpha}=\left(|\alpha|,\left|\alpha^{\prime}\right|+\left|\alpha^{\prime \prime \prime}\right|,\left|\alpha^{\prime \prime}\right|+\left|\alpha^{\prime \prime \prime}\right|,\left|\alpha^{\prime \prime \prime}\right|\right) .
\end{aligned}
$$

Definition A.26. Let $S=S_{j}, j=0,1,2,3$, and $I^{m}(X, S)$ denote the space of all distributions $u \in \mathcal{D}^{\prime}\left(\mathbb{R}^{d}\right)$ such that

$$
u(x)=\int e^{i x \xi} a(x, \xi) d \xi+v(x), \quad v \in C^{\infty}\left(\mathbb{R}^{d}\right), \quad a \in \tilde{S}\left(L, m^{\prime}\right),
$$

where $m_{j}^{\prime}=m_{j}+\left(d / 4-N_{j} / 2\right), N_{0}=d^{\prime}+d^{\prime \prime}+d^{\prime \prime \prime}, N_{1}=d^{\prime}+d^{\prime \prime \prime}, N_{2}=d^{\prime \prime}+d^{\prime \prime \prime}$ and $N_{3}=d^{\prime \prime \prime}$. We write

$$
I(X, S)=\bigcup_{m \in \mathbb{R}^{4}} I^{m}(X, S)
$$

Since $w_{m}(\xi) \leq w_{p}(\xi)$ if $m \leq p$, we obtain $I^{m}(X, S) \subseteq I^{p}(X, S)$ if and only if $m_{j} \leq$ $p_{j}, j=0,1,2,3$.

Definition A.27. Let denote $\mathcal{V}(S)$ the set of all smooth vector fields on $\mathbb{R}^{d}$ which are tangent to all $S_{j}, j=0,1,2,3$.

Using Taylor's expansion, one easily verifies the following result:

Proposition A.28. The set $\mathcal{V}(S)$ is generated by the following vector fields

$$
\begin{aligned}
x^{\prime} \partial_{x^{\prime}} & :=\left\{x_{j} \partial_{x_{k}}, 1 \leq j, k \leq d^{\prime}\right\} \\
x^{\prime \prime} \partial_{x^{\prime \prime}} & :=\left\{x_{j} \partial_{x_{k}}, d^{\prime}<j, k \leq d^{\prime}+d^{\prime \prime}\right\} \\
x^{\prime \prime \prime} \partial_{x} & :=\left\{x_{j} \partial_{x_{k}}, d^{\prime}+d^{\prime \prime}<j \leq d^{\prime}+d^{\prime \prime}+d^{\prime \prime \prime}, 1 \leq k \leq d\right\} \\
\partial_{x^{(4)}} & :=\left\{\partial_{x_{k}}, \quad d^{\prime}+d^{\prime \prime}+d^{\prime \prime \prime}<k \leq d\right\} .
\end{aligned}
$$

Lemma A.29. Let $s \in \mathbb{R}$ and $u \in H_{\mathrm{loc}}^{s}\left(\mathbb{R}^{d}\right)$ such that $V_{1} \ldots V_{N} u \in H_{\mathrm{loc}}^{s}\left(\mathbb{R}^{d}\right)$ for all $N>0$, $V_{1}, \ldots, V_{N} \in \mathcal{V}(L)$. Furthermore, assume that $u$ has compact support. Then $a\left(x^{(4)}, \xi\right)=$ $\int \hat{u}(\xi) d \xi^{(4)} \in S(L, m)$ for all $m \in \mathbb{R}^{4}$ such that $s+\min _{0 \leq j \leq 3}\left\{m_{j}\right\}>0$. 
Proof. For $\alpha=\left(\alpha^{\prime}, \alpha^{\prime \prime}, \alpha^{\prime \prime \prime}, \alpha^{(4)}\right) \in \mathbb{N}^{d}$, set

$$
\begin{aligned}
T(\alpha):=\left\{\beta \in \mathbb{N}^{d}:\left|\beta^{\prime \prime \prime}\right| \leq\left|\alpha^{\prime \prime \prime}\right|,\left|\beta^{\prime}\right|+\left|\beta^{\prime \prime \prime}\right|\right. & \leq\left|\alpha^{\prime}\right|+\left|\alpha^{\prime \prime \prime}\right|, \\
\left|\beta^{\prime \prime}\right|+\left|\beta^{\prime \prime \prime}\right| & \left.\leq\left|\alpha^{\prime \prime}\right|+\left|\alpha^{\prime \prime \prime}\right|,\left|\beta^{\prime}, \beta^{\prime \prime}, \beta^{\prime \prime \prime}\right| \leq\left|\alpha^{\prime}, \alpha^{\prime \prime}, \alpha^{\prime \prime \prime}\right|\right\} .
\end{aligned}
$$

By induction, one easily proves that for all $\beta \in T(\alpha)$,

$$
D_{x}^{\beta} x^{\alpha} \in \sum_{k=0}^{|\beta|} \mathcal{V}^{k} \text {, }
$$

where $\mathcal{V}^{0}$ is the multiplication by smooth functions, $\mathcal{V}^{1}=\mathcal{V}$ and $\mathcal{V}^{k+1}=\mathcal{V} \circ \mathcal{V}^{k}$.

Hence, if $\beta \in T(\alpha)$, then $D^{\beta} x^{\alpha} u=V_{1} \ldots V_{N} u \in H_{l o c}^{s}\left(\mathbb{R}^{d}\right)$. Because supp $u$ is compact and $u \in H_{\mathrm{loc}}^{s}\left(\mathbb{R}^{d}\right)$, we obtain $D^{\alpha} \hat{u} \in\langle\xi\rangle^{-s} L^{2}\left(\mathbb{R}^{d}\right)$ for all $\alpha$.

By assumption of $u$, we have $\xi^{\beta}\left(D^{\alpha} \hat{u}\right) \in\langle\xi\rangle^{-s} L^{2}\left(\mathbb{R}^{d}\right)$ for all $\beta \in T(\alpha)$. Fix a multiindex $\alpha$ and a positive integer $N$. Expanding $\left(\left\langle\xi^{\prime}\right\rangle^{\alpha^{\prime}}\left\langle\xi^{\prime \prime}\right\rangle^{\left|\alpha^{\prime \prime}\right|}\langle\xi\rangle^{\left|\alpha^{\prime \prime \prime}\right|}\left\langle\xi^{(4)}\right\rangle^{N}\right)^{2}$, we obtain

$$
\left\langle\xi^{\prime}\right\rangle^{\left|\alpha^{\prime}\right|}\left\langle\xi^{\prime \prime}\right\rangle^{\left|\alpha^{\prime \prime}\right|}\langle\xi\rangle^{\left|\alpha^{\prime \prime \prime}\right|}\left\langle\xi^{(4)}\right\rangle^{N} D^{\alpha} \hat{u} \in\langle\xi\rangle^{-s} L^{2}\left(\mathbb{R}^{d}\right) .
$$

We have to show that there exist $m \in \mathbb{R}^{4}$ such that for all $N>0$ and multi-index $\alpha$,

$$
\left|D_{\xi}^{\alpha} \hat{u}(\xi)\right| \leq C_{N} w_{m}(\xi)\left\langle\xi^{\prime}\right\rangle^{-\left|\alpha^{\prime}\right|}\left\langle\xi^{\prime \prime}\right\rangle^{-\left|\alpha^{\prime \prime}\right|}\langle\xi\rangle^{-\left|\alpha^{\prime \prime \prime}\right|}\left\langle\xi^{(4)}\right\rangle^{-N}
$$

Choose $m=\left(m_{0}, m_{1}, m_{2}, m_{3}\right) \in \mathbb{R}^{4}$ such that $s+\min m_{j}>0$. For $|\gamma| \geq d / 2$, we obtain

$$
D_{\xi}^{\gamma}\left(w_{m}(\xi)^{-1}\left\langle\xi^{\prime}\right\rangle^{\alpha^{\prime}}\left\langle\xi^{\prime \prime}\right\rangle^{\left|\alpha^{\prime \prime}\right|}\langle\xi\rangle^{\left|\alpha^{\prime \prime \prime}\right|}\left\langle\xi^{(4)}\right\rangle^{N} D_{\xi}^{\alpha} \hat{u}(\xi)\right) \in \max _{|\delta| \leq|\gamma|}\left|D_{\xi}^{\delta} w_{m}(\xi)^{-1}\right|\langle\xi\rangle^{-s} L^{2}\left(\mathbb{R}^{d}\right) .
$$

On the other hand, $w_{m}(\xi)^{-1}=w_{-m}(\xi) \in S(L,-m)$. So $\left|D^{\gamma} w_{-m}(\xi)\right| \leq C w_{-m}(\xi) \leq C\langle\xi\rangle^{s}$ since $s+\min m_{j}>0$. Hence,

$$
\left|D_{\xi}^{\delta} w_{m}(\xi)^{-1}\right|\langle\xi\rangle^{-s} L^{2}\left(\mathbb{R}^{d}\right) \subset L^{2}\left(\mathbb{R}^{d}\right)
$$

For all $N>0$, by Sobolev embedding theorem, we have

$$
w_{m}(\xi)^{-1}\left\langle\xi^{\prime}\right\rangle^{\left|\alpha^{\prime}\right|}\left\langle\xi^{\prime \prime}\right\rangle^{\left|\alpha^{\prime \prime}\right|}\langle\xi\rangle^{\left|\alpha^{\prime \prime \prime}\right|}\left\langle\xi^{(4)}\right\rangle^{N} D_{\xi}^{\alpha} \hat{u}(\xi) \in L^{\infty}\left(\mathbb{R}^{d}\right)
$$

Therefore, by setting $a\left(x^{(4)}, \xi^{\prime}, \xi^{\prime \prime}, \xi^{\prime \prime \prime}\right)=\int e^{i x^{(4)} \xi^{(4)}} \hat{u}(\xi) d \xi^{(4)}$ we conclude that

$$
u(x)=\int e^{i\left(x^{\prime} \xi^{\prime}+x^{\prime \prime} \xi^{\prime \prime}+x^{\prime \prime \prime} \xi^{\prime \prime \prime}\right)} a\left(x^{(4)}, \xi^{\prime}, \xi^{\prime \prime}, \xi^{\prime \prime \prime}\right) d \xi^{\prime} d \xi^{\prime \prime} d \xi^{\prime \prime \prime}, a \in S(L, m) .
$$


Lemma A.30. Let $a(x, \xi) \in S(L, m)$ for some $m \in \mathbb{R}^{4}$ and $\chi \in C_{c}^{\infty}\left(\mathbb{R}^{d}\right)$ be a smooth function with compact support. Set $u(x)=\chi(x) \int e^{i x \xi} a(x, \xi) d \xi$. Then the Fourier transform $\hat{u}(\eta)=F_{x \rightarrow \eta} u(\eta)$ belongs to the space $S(L, m)$. As a corollary, if $u \in I^{m}(X ; L)$ with compact support, then $\hat{u} \in S(L, \tilde{m})$.

Proof. By definition, we have

$$
\hat{u}(\eta)=(2 \pi)^{-d} \int e^{i x(\xi-\eta)} a(x, \xi) \chi(x) d x d \xi
$$

We now show that $\hat{u}(\eta)$ satisfies the estimates above. Fix any $N>0$ and multi-index $\alpha$, we obtain

$$
\begin{aligned}
\left|D_{\eta}^{\alpha} \hat{u}(\eta)\right| & =C\left|\iint e^{i x(\xi-\eta)}(-x)^{\alpha} a(x, \xi) \chi(x) d x d \xi\right| \\
& =C\left|\iint e^{-i x \xi}\langle\xi\rangle^{-2 k}\left(-\Delta_{x}+1\right)^{k}\left(\chi(x) a^{(\alpha)}(x, \eta+\xi)\right) d x d \xi\right| \\
& \leq C \int(m-\hat{\alpha})(\eta+\xi)\langle\xi\rangle^{-2 k}\left\langle\xi^{(4)}+\eta^{(4)}\right\rangle^{-N} d \xi \\
& \leq C(m-\hat{\alpha})(\eta)\left\langle\eta^{(4)}\right\rangle^{-N} \int|m-\hat{\alpha}|(\xi)\langle\xi\rangle^{-2 k}\left\langle\xi^{(4)}\right\rangle^{N} d \xi \leq C^{\prime}(m-\hat{\alpha})(\eta)\left\langle\eta^{(4)}\right\rangle^{-N}
\end{aligned}
$$

This implies that $\hat{u} \in S(L, m)$. This completes the proof.

Proposition A.31. The space $I(X, S)$ is equal to the set of all $u \in \mathcal{D}^{\prime}(X)$ such that there exists $s \in \mathbb{R}$ satisfying $V_{1} \ldots V_{N} u \in H_{\mathrm{loc}}^{s}\left(\mathbb{R}^{d}\right)$ for all $N$, where $V_{1}, \ldots, V_{N} \in \mathcal{V}(L)$.

Proof. Let $u \in I(X ; L)$ and $m \in \mathbb{R}^{4}$ such that $u(x)=\int e^{i x \xi} a(x, \xi) d \xi, a \in S(L, \tilde{m})$. Take any cut-off function $\chi \in C_{c}^{\infty}\left(\mathbb{R}^{d}\right)$. By Lemma $\widehat{\mathrm{A} .30}, \widehat{\chi u}(\eta) \in S(L, \tilde{m})$. This implies that $\widehat{\chi u} \in H^{s}\left(\mathbb{R}^{d}\right)$ for all $s+\min m_{j}^{\prime}>0$. Hence, $I^{m}(X ; L) \subset H_{\mathrm{loc}}^{s}\left(\mathbb{R}^{d}\right)$ for such $s$. Now take any $V_{1}, \ldots, V_{N} \in \mathcal{V}(L)$. By induction we conclude that $V_{1} \ldots V_{N} u \in I^{m}(X ; L)$. Consequently, $V_{1} \ldots V_{N} u \in H_{\text {loc }}^{s}\left(\mathbb{R}^{d}\right)$. Hence, $u$ is stable under iterated action of the vector fields $\mathcal{V}(L)$.

Conversely, let $u \in \mathcal{D}^{\prime}\left(\mathbb{R}^{d}\right)$ such that $u \in H_{\mathrm{loc}}^{s}\left(\mathbb{R}^{d}\right)$ and for all $N>0, V_{1}, \ldots, V_{N} \in$ $\mathcal{V}(L)$, we have $V_{1} \ldots V_{N} u \in H_{\mathrm{loc}}^{s}\left(\mathbb{R}^{d}\right)$. We have to show that there exists $m \in \mathbb{R}^{4}$ such that $u \in I^{m}(X ; L)$. Take any locally finite family $\left\{\psi_{j}\right\} \subset C_{c}^{\infty}\left(\mathbb{R}^{d}\right)$ such that $\sum \psi_{j}^{2}=1$. Applying Lemma A.29, we obtain

$$
\psi_{j} u=\int e^{i x \xi} a_{j}(\xi) d \xi, a_{j}(\xi) \in S\left(L, m^{\prime}\right)
$$

where $m \in \mathbb{R}^{4}$ such that $s+\min m_{j}^{\prime}>0$. Set $a(x, \xi)=\sum_{j} \psi_{j}(x) a_{j}(\xi)$. Hence, $a \in S\left(L, m^{\prime}\right)$ and $u(x)=\int e^{i x \xi} a(x, \xi) d \xi$. Thus, $u \in I^{m}(X ; L)$. The proof is complete. 
Corollary A.32. If $u \in I(X ; L)$, then $\mathrm{WF}(u) \subseteq L$.

In fact, since $\mathcal{V}(L)^{N} u \in H_{\text {loc }}^{s}\left(\mathbb{R}^{d}\right)$ for all $N \in \mathbb{N}$, we obtain

$$
\mathrm{WF}(u) \subseteq\left\{(x, \xi) \in \mathbb{R}^{d} \times \mathbb{R}^{d} \backslash 0: \sigma(V)(x, \xi)=0, \quad \forall V \in \mathcal{V}(L)\right\}=L .
$$

Note that if $u \in I^{m}(X ; L)$ then $u \in I^{m_{j}}\left(X ; L_{j} \backslash\left(\cup_{k \neq j} L_{k}\right)\right.$ for $j=0,1,2,3$.

Theorem A.33. Let $S=\left\{S_{j}, j=0,1,2,3\right\}$ and $L=\left\{L_{j}, j=0,1,2,3\right\}$ be given as above. Then we have

$$
I(X, S)=I(X ; L) .
$$

Hence, we write $I^{m}(X ; L)=I^{m}(X, S)$.

Proof. The first part $I(X ; L) \subset I(X, S)$ follows from the inclusion $\mathcal{V} \subset \mathrm{Op}(\mathcal{M})$. Hence, it remains to prove that for any operator $P \in \mathrm{Op}(\mathcal{M})$,

$$
P: I^{m}(X ; L) \rightarrow I^{m}(X ; L) \text {. }
$$

We notice that $\$ .39$ holds for all vector fields in $\left\{x^{\prime} \partial_{x^{\prime}}, x^{\prime \prime} \partial_{x^{\prime \prime}}, x^{\prime \prime \prime} \partial_{x}, \partial_{x^{(4)}}\right\}$ which generate $\mathcal{V}$.

Take any $P \in \operatorname{Op}(\mathcal{M})$ and $u \in I^{m}(X ; L)$. Since the principal symbol $\sigma_{0}(P)(x, \xi)$ of $P$ vanishes on $L$, there exist smooth functions $q_{j}(x, \xi /|\xi|)$ such that

$$
\sigma_{0}(P)(x, \xi)=\sum_{j} q_{j}(x, \xi /|\xi|) v_{j}(x, \xi)
$$

where $v_{j}(x, \xi) \in\left\{x^{\prime} \xi^{\prime}, x^{\prime \prime} \xi^{\prime \prime}, x^{\prime \prime \prime} \xi, \xi^{(4)}\right\}$. Let $Q_{j}$ be properly pseudodifferential operators with symbol $q_{j}, V_{j}$ vector fields associated with $v_{j}$. Set $Q_{0}=P-\sum_{j} Q_{j} V_{j} \in \Psi^{1}\left(\mathbb{R}^{d}\right)$. Then the principal symbol of $Q_{0}$ vanishes on a conic neighbourhood of $L$. Thus, $Q_{0} \in \Psi^{0}\left(\mathbb{R}^{d}\right)$ such that $P=Q_{j} V_{j}+Q_{0}$ near $L$. Hence, $P u=\sum Q_{j} V_{j} u+Q_{0} u \in I^{m}(X ; L)$ for $Q_{j}, V_{j}$ act on $I^{m}(X ; L)$.

Similarly, we obtain:

Theorem A.34. Let $P \in \Psi^{p}\left(\mathbb{R}^{d}\right)$ be a properly pseudodifferential operator of order $p$. Then $u \in I^{m}(X ; L)$ implies $P u \in I^{m^{\prime}}(X ; L)$, where $m_{j}^{\prime}=m_{j}+p, j=0,1,2,3$. In particular, the spaces $I^{m}(X ; L), m \in \mathbb{R}$ are invariant under action of the properly supported pseudodifferential operators of order zero.

If $P \in \Psi^{1}\left(\mathbb{R}^{d}\right)$ is a properly supported operators such that its principal symbol $p_{0}(x, \xi)$ vanishes on $L$, then $P u \in I^{m}(X ; L)$. 
Sketch of Proof. Without loss of generality, take $u \in I^{m}(X ; L) \cap \mathcal{E}^{\prime}(X)$. By Lemma A.30, $\hat{u}\left(\xi^{\prime}, \xi^{\prime \prime}, \xi^{\prime \prime \prime}, x^{(4)}\right) \in S\left(L, m^{\prime}\right)$. Let $p(x, \xi) \in S^{p-d / 4}\left(\mathbb{R}^{d} \times \mathbb{R}^{d}\right)$ be the total left symbol of $P \in \Psi^{p}\left(\mathbb{R}^{d}\right)$, then $P u=\int e^{i x \xi} p(x, \xi) \hat{u}(\xi) d \xi$. Because $p \in S^{p-d / 4}$ and $\hat{u} \in S\left(L, m^{\prime}\right)$, $p(x, \xi) \hat{u}(\xi) \in S(L, \tilde{m}+p)$. Hence, $P u \in I^{m^{\prime}}(X ; L)$, with $m^{\prime}=\left(m_{0}+p, m_{1}+p, m_{2}+p, m_{3}+p\right)$. The proof is complete.

Similarly, we now define the class of distributions associated with three Lagrangian manifolds $L_{0}, L_{2}, L_{3}$ :

Definition A.35. Let $S^{m_{0}, m_{2}, m_{3}}\left(L_{0}, L_{2}, L_{3}\right)$ be the set of all smooth functions $a(x, \xi)$ such that for any multi-indices $\alpha, \beta$ and $K \subset \subset \mathbb{R}^{d}$, there is a constant $C_{K, \alpha, \beta}>0$ such that

$$
\left|D_{x}^{\beta} D_{\xi}^{\alpha} a(x, \xi)\right| \leq C\left\langle\xi^{\prime}\right\rangle^{m_{0}-m_{2}-\left|\alpha^{\prime}\right|}\left\langle\xi^{\prime}, \xi^{\prime \prime}\right\rangle^{m_{2}-m_{3}-\left|\alpha^{\prime \prime}\right|}\langle\xi\rangle^{m_{3}-\left|\alpha^{\prime \prime \prime}\right|}, \quad x \in K .
$$

We denote by $I^{m_{0}, m_{2}, m_{3}}\left(X ; L_{0}, L_{2}, L_{3}\right)$ the set of all $u \in \mathcal{D}^{\prime}\left(\mathbb{R}^{d}\right)$ such that

$$
u(x)=\int e^{i\left(x^{\prime} \xi^{\prime}+x^{\prime \prime} \xi^{\prime \prime}+x^{\prime \prime \prime} \xi^{\prime \prime \prime}\right)} a(x, \xi) d \xi, a(x, \xi) \in S^{m_{0}^{\prime}, m_{2}^{\prime}, m_{3}^{\prime}}\left(L_{0}, L_{2}, L_{3}\right),
$$

where $m_{0}^{\prime}=m_{0}+d / 4-\left(d^{\prime}+d^{\prime \prime}+d^{\prime \prime \prime}\right) / 2, m_{2}=m_{2}+d / 4-\left(d^{\prime \prime}+d^{\prime \prime \prime}\right) / 2, m_{3}^{\prime}=$ $m_{3}+d / 4-d^{\prime \prime \prime} / 2$.

Theorem A.36. Let $L_{0}, L_{2}, L_{3}$ be given as above. Then the space $\bigcup_{m} I^{m_{0}, m_{2}, m_{3}}\left(X ; L_{0}, L_{2}, L_{3}\right)$ consists of all $u \in \mathcal{D}^{\prime}(X)$ such that there exists a $s \in \mathbb{R}$ satisfying

$$
P_{1} \ldots P_{N} u \in H_{\mathrm{loc}}^{s}\left(\mathbb{R}^{d}\right), \quad \forall N \in \mathbb{N}
$$

where $P_{j}, 1 \leq j \leq N$ are first order, properly supported pseudodifferential operators whose principal symbols vanish on $L_{0} \cup L_{2} \cup L_{3}$.

The relations of these spaces with four-Lagrangian distributions are given in the following result.

Proposition A.37. For every $\left(m_{0}, m_{2}, m_{3}\right) \in \mathbb{R}^{3}$, we have

$$
I^{\left(m_{0},-\infty, m_{2}, m_{3}\right)}(X ; L) \subsetneq I^{m_{0}, m_{2}, m_{3}}\left(X ; L_{0}, L_{2}, L_{3}\right) \subset I^{m_{0}, m_{1}, m_{2}, m_{3}}(X ; L),
$$

where $m_{1}=m_{0}-d^{\prime \prime} / 2$.

Proof. Let $m_{1}=m_{0}-d^{\prime \prime} / 2$. Then $m_{1}^{\prime}=m_{0}^{\prime}$. Hence, we have

$$
\begin{aligned}
\left\langle\xi^{\prime}\right\rangle^{m_{0}^{\prime}-m_{2}^{\prime}-\left|\alpha^{\prime}\right|}\left\langle\xi^{\prime}, \xi^{\prime \prime}\right\rangle^{m_{2}^{\prime}-m_{3}^{\prime}-\left|\alpha^{\prime \prime}\right|}\langle\xi\rangle^{m_{3}^{\prime}-\left|\alpha^{\prime \prime \prime}\right|} & =w_{m^{\prime}}(\xi)\left(\frac{\left\langle\xi^{\prime}, \xi^{\prime \prime}\right\rangle}{\left\langle\xi^{\prime \prime}\right\rangle}\right)^{m_{0}^{\prime}-m_{1}^{\prime}-\left|\alpha^{\prime \prime}\right|}\left\langle\xi^{\prime}\right\rangle^{-\left|\alpha^{\prime}\right|}\left\langle\xi^{\prime \prime}\right\rangle^{-\left|\alpha^{\prime \prime}\right|}\langle\xi\rangle^{-\left|\alpha^{\prime \prime \prime}\right|} \\
& \leq w_{m^{\prime}}(\xi)\left\langle\xi^{\prime}\right\rangle^{-\left|\alpha^{\prime}\right|}\left\langle\xi^{\prime \prime}\right\rangle^{-\left|\alpha^{\prime \prime}\right|}\langle\xi\rangle^{-\left|\alpha^{\prime \prime \prime}\right|} .
\end{aligned}
$$


Thus, if $a \in S^{m_{0}^{\prime}, m_{2}^{\prime}, m_{3}^{\prime}}\left(L_{0} \cdot L_{1}, L_{3}\right)$ then $a \in S\left(L, w_{m^{\prime}}\right)$. Therefore, the right inclusion follows. The left inclusion is trivial.

Remark A.38. As a consequence, if $a(x, \xi) \in S\left(L, w_{m}\right)$ satisfies

$$
\left|\xi^{\prime}\right| \leq\left|\xi^{\prime \prime}\right|, \quad \forall(x, \xi) \in \operatorname{supp} a
$$

then $a \in S^{m_{0}, m_{2}, m_{3}}\left(L_{0}, L_{2}, L_{3}\right)$.

Theorem A.39. For all $m \in \mathbb{R}^{4}$, we have

$$
I^{m}(X ; L) \subset I^{m_{0}, m_{1}, m_{3}}\left(X ; L_{0}, L_{1}, L_{3}\right)+I^{m_{0}, m_{2}, m_{3}}\left(X ; L_{0}, L_{2}, L_{3}\right) \subset I^{m^{*}}(X ; L),
$$

where $m^{*}=\left(m_{0}, \max \left\{m_{1}, m_{0}-d^{\prime \prime} / 2\right\}, \max \left\{m_{2}, m_{0}-d^{\prime} / 2\right\}, m_{3}\right)$.

In particular, if $\min \left\{m_{1}+d^{\prime \prime} / 2, m_{2}+d^{\prime} / 2\right\} \geq m_{0}$, then the following decomposition holds

$$
I^{m}(X ; L)=I^{m_{0}, m_{1}, m_{3}}\left(X ; L_{0}, L_{1}, L_{3}\right)+I^{m_{0}, m_{2}, m_{3}}\left(X ; L_{0}, L_{2}, L_{3}\right) .
$$

Proof. Let $\chi \in C^{\infty}(\mathbb{R})$ be a smooth function satisfying

$$
\chi(t)= \begin{cases}0, & \text { for } t<1, \\ 0 \leq \chi(t) \leq 1, & \text { for } 1 \leq t \leq 2, \\ 1, & \text { for } t>2 .\end{cases}
$$

Then $\phi\left(\xi^{\prime}, \xi^{\prime \prime}\right)=\chi\left(\left\langle\xi^{\prime}\right\rangle /\left\langle\xi^{\prime \prime}\right\rangle\right)$ has support in $\left\langle\xi^{\prime}\right\rangle \geq\left\langle\xi^{\prime \prime}\right\rangle$, and for all $\alpha^{\prime}, \alpha^{\prime \prime}$, there exists a constant $C\left(\alpha^{\prime}, \alpha^{\prime \prime}\right)$ such that

$$
\left|\mathcal{D}_{\xi^{\prime}}^{\alpha^{\prime}} D_{\xi^{\prime \prime}}^{\alpha^{\prime \prime}} \phi\left(\xi^{\prime}, \xi^{\prime \prime}\right)\right| \leq C\left\langle\xi^{\prime}\right\rangle^{-\left|\alpha^{\prime}\right|}\left\langle\xi^{\prime \prime}\right\rangle^{-\left|\alpha^{\prime \prime}\right|}
$$

Therefore, if $u(x)=\int e^{i x \xi} a(x, \xi) d \xi, a \in S\left(L, m^{\prime}\right)$ then $u=u_{1}+u_{2}$, where

$$
\begin{aligned}
& u_{1}(x)=\int e^{i x \xi} a_{1}(x, \xi) d \xi, a_{1}(x, \xi)=\phi\left(\xi^{\prime}, \xi^{\prime \prime}\right) a(x, \xi) \\
& u_{2}(x)=\int e^{i x \xi} a_{1}(x, \xi) d \xi, a_{2}(x, \xi)=\left(1-\phi\left(\xi^{\prime}, \xi^{\prime \prime}\right)\right) a(x, \xi) .
\end{aligned}
$$

Since $a \in S\left(L, m^{\prime}\right)$, it is obvious that $a_{1} \in S^{m^{\prime}}\left(L_{0}, L_{1}, L_{3}\right)$ and in the support of $a_{1}$ we have $\left|\xi^{\prime}\right| \geq\left|\xi^{\prime \prime}\right|$. This implies that $a_{1} \in S^{m_{0}^{\prime}, m_{1}^{\prime}, m_{3}^{\prime}}\left(L_{0}, L_{1}, L_{3}\right)$. Similarly, $a_{2} \in S\left(L, m^{\prime}\right)$ and $\left\langle\xi^{\prime}\right\rangle \leq 2\left\langle\eta^{\prime \prime}\right\rangle$ in the support of $a_{2}$. This shows that $\in S^{m_{0}^{\prime}, m_{2}^{\prime}, m_{3}^{\prime}}\left(L_{0}, L_{2}, L_{3}\right)$. Hence, $u_{j}(x) \in I^{m_{0}, m_{j}, m_{3}}\left(X ; L_{0}, L_{j}, L_{3}\right), j=1,2$. Thus, the left inclusion in the theorem holds. The right one follows from Proposision A.37. The proof is complete. 
Definition A.40. Let $J$ be a subset of $\{0,1,2,3\}$. Denote $\mathcal{V}\left(S_{j}, j \in J\right)$ the set of all smooth vector fields on $\mathbb{R}^{d}$ which are tangent to $S_{j}, j \in J$. Similarly, $\operatorname{Op}\left(\mathcal{M}\left(L_{j}, j \in J\right)\right)$ stands for the set of all classical properly first order pseudodifferential operators on $\mathbb{R}^{d}$ with principal symbols vanishing on $\cup_{j \in J} L_{j}$.

In general, $\mathcal{V}\left(S_{j}, j \in J\right)$ is a subspace of the vector space $\operatorname{Op}\left(\mathcal{M}\left(S_{j}, j \in J\right)\right)$. In some case, $\mathrm{Op}\left(\mathcal{M}\left(S_{j}, j \in J\right)\right)$ are generated by $\mathcal{V}\left(S_{j}, j \in J\right)$ over $\Psi^{0}\left(\mathbb{R}^{d}\right)$. For example, we have

$$
\begin{array}{rr}
\mathcal{V}\left(S_{0}, S_{1}, S_{2}, S_{3}\right)=\operatorname{span}\left\{\partial_{x^{4}}, x^{\prime} \partial_{x^{\prime}}, x^{\prime \prime} \partial_{x^{\prime \prime}}, x^{\prime \prime \prime} \partial_{x}\right\} & \\
\mathcal{V}\left(S_{0}, S_{1}, S_{3}\right)=\operatorname{span}\left\{\partial_{x^{4}}, x^{\prime \prime \prime} \partial_{x},\left(x^{\prime}, x^{\prime \prime}\right) \partial_{x^{\prime \prime}}, x^{\prime} \partial_{x^{\prime}}\right\} & =\left\{x^{\prime} \partial_{x^{\prime \prime}}\right\} \cup \mathcal{V} \\
\mathcal{V}\left(S_{0}, S_{2}, S_{3}\right)=\operatorname{span}\left\{\partial_{x^{4}}, x^{\prime \prime \prime} \partial_{x}, x^{\prime \prime} \partial_{x^{\prime \prime}},\left(x^{\prime}, x^{\prime \prime}\right) \partial_{x^{\prime}}\right\} & =\left\{x^{\prime \prime} \partial_{x^{\prime}}\right\} \cup \mathcal{V} \\
\mathcal{V}\left(S_{0}, S_{1}\right)=\operatorname{span}\left\{\partial_{x^{4}}, x^{\prime \prime \prime} \partial_{x}, x^{\prime \prime} \partial_{x^{\prime \prime}}, x^{\prime} \partial_{x}\right\} & \left.=\left\{x^{\prime} \partial_{x^{\prime \prime}}, x^{\prime} \partial_{x^{\prime \prime \prime}}\right\} \cup \mathcal{V} \partial_{x^{\prime}}, x^{\prime \prime} \partial_{x^{\prime \prime \prime}}\right\} \cup \mathcal{V} \\
\mathcal{V}\left(S_{0}, S_{2}\right)=\operatorname{span}\left\{\partial_{x^{4}}, x^{\prime \prime \prime} \partial_{x}, x^{\prime \prime} \partial_{x}, x^{\prime} \partial_{x^{\prime}}\right\} & =\left\{x^{\prime} x^{\prime \prime} \partial_{x}\right\} \cup \mathcal{V} \\
\mathcal{V}\left(S_{1}, S_{2}\right)=\operatorname{span}\left\{\partial_{x^{4}}, x^{\prime \prime \prime} \partial_{x}, x^{\prime \prime} \partial_{x^{\prime \prime}}, x^{\prime} \partial_{x^{\prime}}, x^{\prime} x^{\prime \prime} \partial_{x}\right\} & =\left\{x^{\prime} x^{\prime \prime} \partial_{x}\right\} \cup \mathcal{V} \\
\mathcal{V}\left(S_{0}, S_{1}, S_{2}\right)=\operatorname{span}\left\{\partial_{x^{4}}, x^{\prime \prime \prime} \partial_{x}, x^{\prime \prime} \partial_{x^{\prime \prime}}, x^{\prime} \partial_{x^{\prime}}, x^{\prime} x^{\prime \prime} \partial_{x}\right\} & =\mathcal{V}, \\
\mathcal{V}\left(S_{1}, S_{2}, S_{3}\right)=\operatorname{span}\left\{\partial_{x^{4}}, x^{\prime} \partial_{x^{\prime}}, x^{\prime \prime} \partial_{x^{\prime \prime}}, x^{\prime \prime \prime} \partial_{x}\right\} &
\end{array}
$$

The following relations hold:

$$
\begin{aligned}
\mathrm{Op}(\mathcal{M}(L)) & =\Psi^{0}(X) \mathcal{V}(L)+\Psi^{0}(X) \\
\mathrm{Op}\left(\mathcal{M}\left(L_{1}, L_{2}\right)\right) & =\Psi^{0}(X)\left(\mathcal{V}\left(S_{1}, S_{2}\right)+\mathrm{Op}\left(\xi^{\prime} \xi^{\prime \prime} /|\xi|\right)\right)+\Psi^{0}(X) \\
\mathrm{Op}\left(\mathcal{M}\left(L_{0}, L_{1}, L_{2}\right)\right) & =\Psi^{0}(X) \mathcal{V}\left(S_{0}, S_{1}, S_{2}\right)+\Psi^{0}(X) \\
\mathrm{Op}\left(\mathcal{M}\left(L_{1}, L_{2}, L_{3}\right)\right) & =\Psi^{0}(X)\left(\mathcal{V}\left(S_{1}, S_{2}, S_{3}\right)+\mathrm{Op}\left(\xi^{\prime} \xi^{\prime \prime} /|\xi|\right)\right)+\Psi^{0}(X) .
\end{aligned}
$$

Therefore, $\mathcal{M}(\cdot)$ is finite generated over the ring $\Psi^{0}$ of first order, classical, properly supported pseudodifferential operators on $X$. Note that the operators $\operatorname{Op}\left(\xi^{\prime} \xi^{\prime \prime} /|\xi|\right)$ kill the symbols on $L_{0}$ while operators $\operatorname{Op}\left(x^{\prime} x^{\prime \prime} \xi_{j}\right), 1 \leq j \leq d$ kill the symbols on $L_{3}$. 


\section{Bibliography}

[AU85] J. Antoniano and G. Uhlmann, A functional calculus for a class of pseudodifferential operators with singular symbols, Proc. Symp Pure Math 43 (1985), $5-16$.

[BG81] L. Boutet de Monvel and V. Guillemin, The spectral theory of Toeplitz operators, Princeton 1981.

[Cha74] Chazarain, J., Opérateurs hyperboliques à caractéristiques de multiplicité constante, Ann. 1974.

[Dui96] J. J. Duistermaat, Fourier integral operators, Birkhäuser, Boston, 1996.

[DG75] J. J. Duistermaat and V. Guillemin, The spectrum of positive elliptic operators and periodic bicharacteristics, Inventiones mathematicae 29 (1975), 39-79.

[DH72] J. J. Duistermaat and L. Hörmander, Fourier integral operators II, Acta. Math. , 128 (1972), 183-269.

[DK00] J. J. Duistermaat and J. A. C. Kolk, Lie groups, Berlin: Springer-Verlag 2000.

[FGr08] R. Felea and A. Greenleaf, An FIO Calculus for Marine Seismic Imaging: Folds and Cross Caps, Comm. PDE. 33 (2008), 45-77.

[GrU90] A. Greenleaf and G. Uhlmann, Estimates for singular Radon transforms and pseudodifferential operators with singular symbols, J. Funct. Anal. 89 (1990), 202-232.

[GrU91] A. Greenleaf and G. Uhlmann, Composition of some singular Fourier integral operators and estimates for restricted. X-ray transforms, I. Ann. Inst. Fourier 40, 443-466 (1990); II, Duke Math. J. 64, 415-444 (1991)

[GrU93] A. Greenleaf and G. Uhlmann, Recovering Singularities of a Potential from Singularities of Scattering Data, Comm. in Math. Physics, Vol. 157, Issue 3, 549-572.

[GS77] V. Guillemin and S. Sternberg, Geometric Asymptotics, Mathematical Surveys and Monographs, vol. 14, Providence, Rhode Island, 1977.

[GU81] V. Guillemin and G. Uhlmann, Oscillatory integrals with singular symbols, Duke Math. J. 48 (1981), 251-267. 
[Hör71] L. Hörmander, Fourier Integral Operators I, Acta Math. 127 (1971), 79-183.

[Hör85] L. Hörmander, The Analysis of Linear Partial Differential Operators, volume I-IV. Grundlehren, Springer, 256, 257, 274, 275.

[Hör97] L. Hörmander, Lecture notes on nonlinear hyperbolic differential equations. Springer 1997.

[Jos94] M. S. Joshi, A precise calculus of paired lagrangian distributions, MIT thesis, 1994.

[Jos98] M. S. Joshi, A symbolic construction of the forward fundamental solution of the wave operator, Comm. PDE. 23 (1998), 1349-1417.

[Jos99] M. S. Joshi, Geometric proofs of composition theorems for generalized Fourier integral operators, Portugaliae Mathematica, Vol. 56 Fasc. 2-1999.

[LW94] P. Laubin and B. Willems, Distributions associated to a 2-microlocal pair of lagrangian manifolds, Comm. PDE. 19 (1994), 1581-1610.

[Lax57] Lax P. D., Asymptotic Solutions of Oscillatory Initial Value Problems, Duke Math. J. 24(1957), 627-646.

[Lax06] Lax, P. D., Hyperbolic partial differential equations. Providence, R.I. , 2006.

[Lud60] Ludwig, D., Exact and asymptotic solutions of the Cauchy problem, Comm. Pure Appl. Math. 13 (1960), 473-508.

[MU79] R. Melrose and G. Uhlmann, Lagrangian intersection and the Cauchy problem, Comm. Pure Appl. Math. 32 (1979), 483-519.

[Mel87] R. Melrose, Marked lagrangians, notes of lectures at Max Planck Institut, 1987.

[Men82] G. Mendoza, Symbol calculus associated with intersecting lagrangians, Comm. PDE. 7 (1982), 1035-1116.

[NS04] V. Nazaikinskii and B. Sternin, Relative elliptic theory. In: J. Gil, T. Krainer, and I. Witt (eds.), Aspects of boundary problems in analysis and geometry, Oper. Theory Adv. Appl. vol. 151, Birkhäuser, Basel, 2004, pp. 495-560.

[Tre80] F. Treves, Introduction to Pseudodifferential and Fourier integral operators, Vol. 2, The university series in Mathematics, 1980. 